University of Rhode Island

DigitalCommons@URI

Open Access Dissertations

2019

\title{
ANTI-INFLAMMATORY EFFECTS OF POLYPHENOL-ENRICHED EXTRACTS AND THEIR GUT MICROBIAL METABOLITES
}

Nicholas A. DaSilva

University of Rhode Island, nickdasilva91@gmail.com

Follow this and additional works at: https://digitalcommons.uri.edu/oa_diss

\section{Recommended Citation}

DaSilva, Nicholas A., "ANTI-INFLAMMATORY EFFECTS OF POLYPHENOL-ENRICHED EXTRACTS AND THEIR GUT MICROBIAL METABOLITES" (2019). Open Access Dissertations. Paper 868.

https://digitalcommons.uri.edu/oa_diss/868

This Dissertation is brought to you for free and open access by DigitalCommons@URI. It has been accepted for inclusion in Open Access Dissertations by an authorized administrator of DigitalCommons@URI. For more information, please contact digitalcommons-group@uri.edu. 


\section{ANTI-INFLAMMATORY EFFECTS OF POLYPHENOL-ENRICHED EXTRACTS \\ AND THEIR GUT MICROBIAL METABOLITES}

BY

NICHOLAS A. DASILVA

ABBREVIATED TITLE: ANTI-INF PROPS OF GMMS FROM PE AND MSX

A DISSERTATION SUBMITTED IN PARTIAL FULFILLMENT OF THE REQUIREMENTS FOR THE DEGREE OF DOCTOR OF PHILOSOPHY

IN

PHARMACEUTICAL SCIENCES

UNIVERSITY OF RHODE ISLAND

2019 


\section{DOCTOR OF PHILOSOPHY DISSERTATION}

OF

NICHOLAS A. DASILVA

APPROVED:

\section{Dissertation Committee}

Major Professor

Co-Major Professor

Nisanne Ghonem

Samantha Meenach

Nasser H. Zawia

Dean, The Graduate School - URI

UNIVERSITY OF RHODE ISLAND

2019 


\begin{abstract}
Background: Chronic inflammation is linked to the onset of diabetes, heart disease, liver disease, neurological diseases and even certain cancers. In the brain, inflammation activates and promotes recruitment of microglia (i.e. reactive microgliosis) and is implicated in the pathogenesis of a number of neurodegenerative diseases. Inflammatory stimuli can cause glucose/insulin intolerance, disrupt liver lipid transport, metabolism and catabolism leading to pre-mature liver failure and cancer. A standardized Pomegranate extract (PE) and Maple Syrup Extract (MSX) could be a source of beneficial phytonutrients capable of mitigating these inflammatory processes.
\end{abstract}

Methods: A pilot scale diet-induced obesity study with Male C57BL/6 mice with or without PE or MSX for 12 weeks was used to probe if PE or MSX could prevent inflammation within the hippocampus. A larger C57BL/6 mouse modeling a typical western diet was used to further evaluate MSX for its ability to modulate inflammation and metabolic syndrome progression. Finally, a human macrophage study modeling systemic inflammation and a co-culture system of murine microglia and human neurons modeling the microenvironment within the brain were used to explore anti-inflammatory effects of PE metabolites (urolithins) and MSX.

Results and Conclusions: Mice fed a HFD for 12 weeks showed elevated expression of both pro and anti-inflammatory cytokine/growth factors and neurodegenerative associated genes in hippocampal tissues. PE in HFD reduced the gene expression of IL-1 $\alpha$, IL-1 $\beta$, IL-7, IL-11, TNF $\alpha$, MAPT, APP, GSK3 3 , and LEPR. MSX in HFD feed decreased the gene expression of IL-7, IL-11, IL19, TNF $\alpha$, IFN $\alpha$, CD40L, CD70, NFE2L2, SOD1, ITGAM, and LEPR. In a western diet model, MSX increased liver lipids, decreased white adipose tissue weight and serum levels of IL-6. In human macrophages, MSX reduced cytokine release. In a co-culture neuroinflammation model, Urolithins and MSX prevented reactive microgliosis and neuronal cell death 


\section{ACKNOWLEDGEMENTS}

First and foremost, I must thank my parents, Antonio and Maria Raposo-DaSilva, for without their encouragement to pursue my PhD and motivation over the years; I would not be where I am today. To my wife, Stephanie DaSilva, I thank you for standing by me throughout my course of study, many weekend-long experiments, late nights of writing, and during my animal study. Your patience, encouragement, and love kept me on track to make me not only a better scientist but a better husband. To my family members, I greatly appreciate your support, motivation, and care during my time in graduate school.

To my labmates, colleagues, undergraduates, and friends, I thank you for the amazing community and support system we have formed over the years which I truly believe will continue to grow and flourish over the course of our careers.

To my advisors, Drs. Navindra Seeram and Angela Slitt, you have been my guides through this entire process. Not to be understated, I sincerely thank you for the opportunities you have given me. Your patience and support over the years have pushed me to grow professionally. Despite the failures and frustration associated with science, you both have shared with me your mindset and techniques to be successful in whichever career path I may take. I would also like to thank my committee members, Dr. Nisanne Ghonem, Dr. Samantha Meenach, and Dr. Brietta Oaks for lending their expertise and training to fully shape this body of work. 


\section{DEDICATION}

This body of work is dedicated to my late grandparents,

Luis F. Raposo and Maria I. DaSilva.

Their love, support, work ethic, and the countless other values they instilled in me throughout my life will not be forgotten. Their sacrifices in life continue to push me and my efforts in science and beyond. 


\section{PREFACE}

The following body of work is presented in manuscript format which constitute three major chapters. The publication statuses of each manuscript are as follows:

Chapter 1: Effects of Pomegranate and Maple Syrup Extracts on Diet-Induced Obesity and Neuroinflammation

Manuscript 1: In Preparation for submission to Nutritional Metabolism

Manuscript 2: In Preparation for submission to Nutritional Neuroscience

\section{Chapter 2: Metabolic Syndrome and Maple Syrup Extract}

Manuscript 3: In Preparation for submission to Molecular Metabolism

\section{Chapter 3: Neuroprotective effects of Pomegranate and Maple Syrup Extracts}

Manuscript 4: Published in Nutritional Neuroscience (2017)

Manuscript 5: Published in Neurochemical Research (2016) 


\section{TABLE OF CONTENTS}

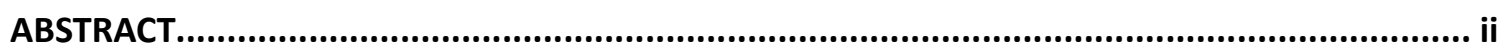

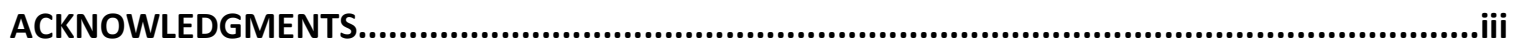

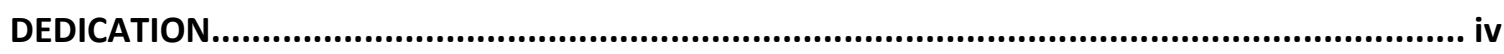

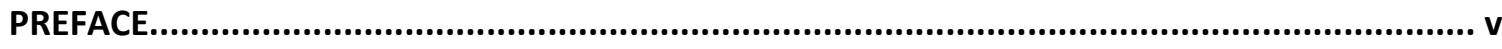

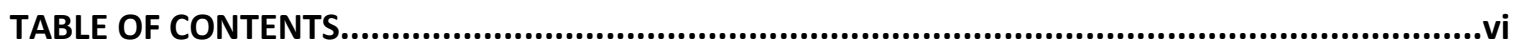

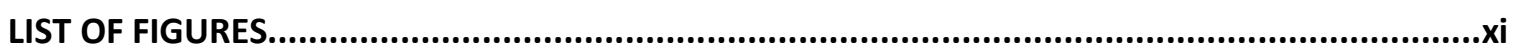

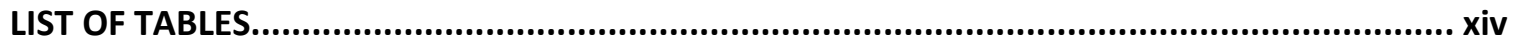

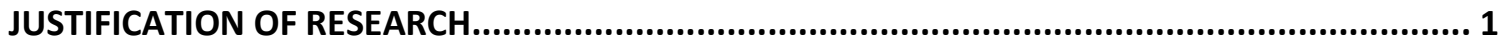

CHAPTER 1: Effects of Pomegranate and Maple Syrup Extracts on Diet-Induced Obesity and Neuroinflammation

Manuscript 1: Anti-inflammatory and neuroprotective effects of a standardized pomegranate

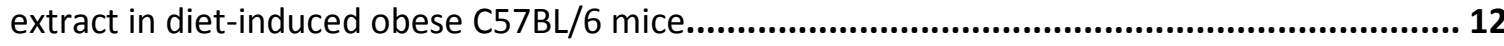

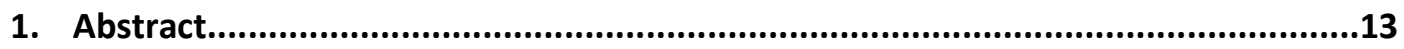

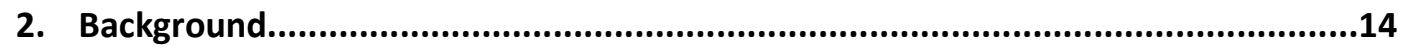

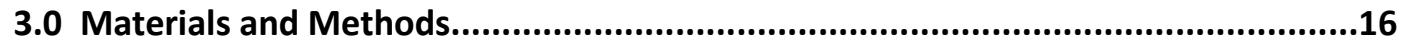

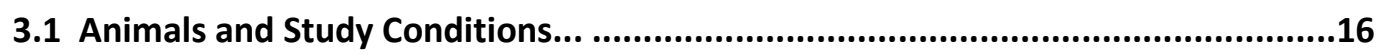

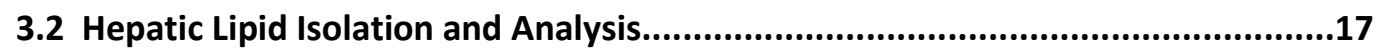

3.3 Nucleic Acid Isolation from Tissue...................................................................18

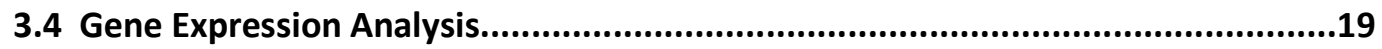

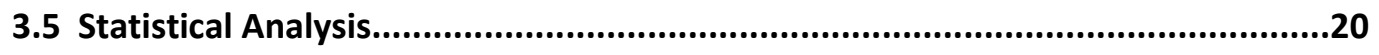

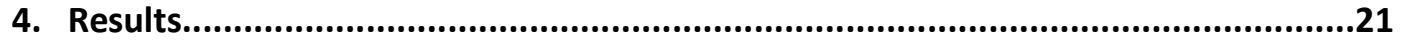

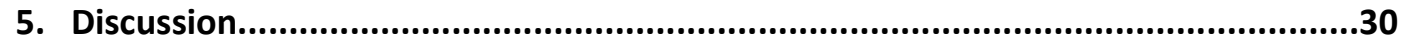

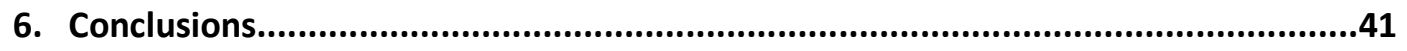

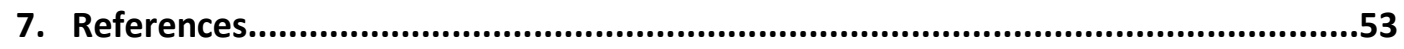


Manuscript 2: Effects of a food grade, phenol-enriched maple syrup extract on hippocampus tissue from diet-induced obese $\mathrm{C} 57 \mathrm{BL} / 6$ mice.

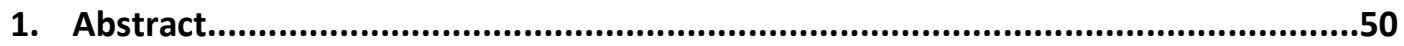

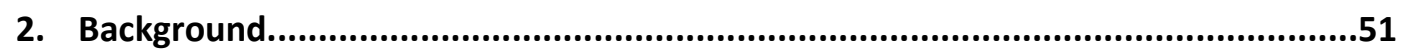

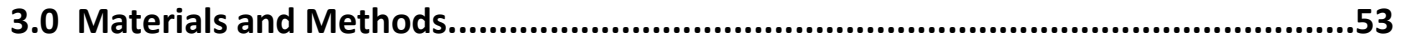

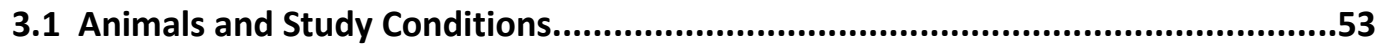

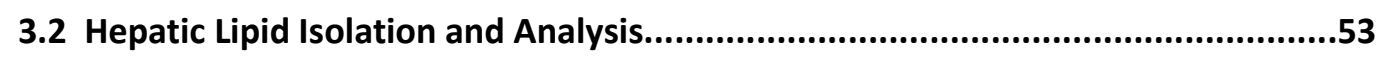

3.3 Nucleic Acid Isolation from Tissue...................................................................54

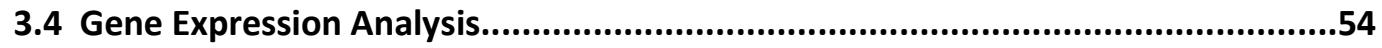

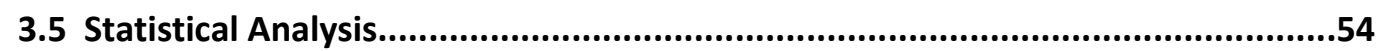

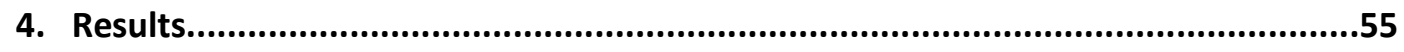

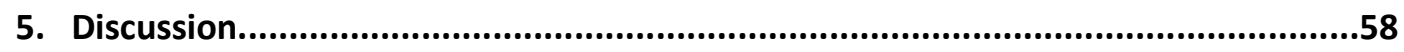

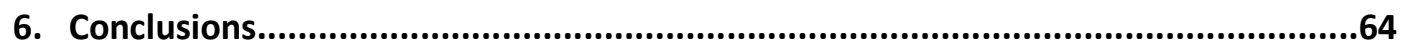

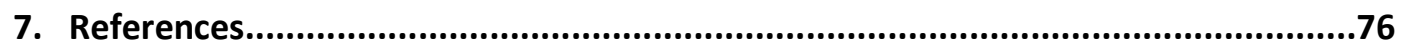




\section{CHAPTER 2: Metabolic Syndrome and Maple Syrup Extract}

Manuscript 3: Evaluation of a food grade maple syrup extract against western diet-induced obesity in male C57BL/6 mice.

1. Abstract..

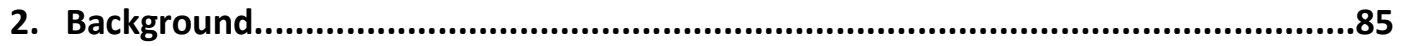

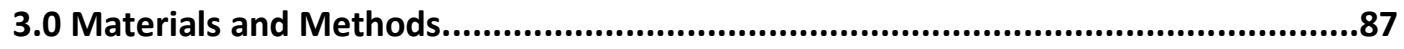

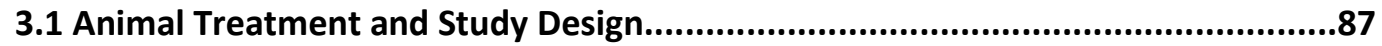

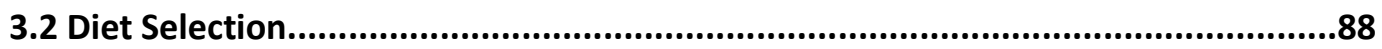

3.3 Glucose Tolerance Test (GTT) and Insulin Tolerance Test (ITT)...........................88

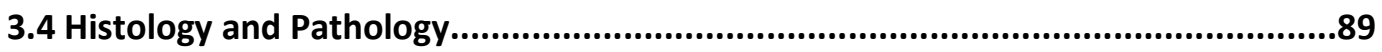

3.5 Cell Culture of Human Monocytes and Macrophages.........................................89

3.6 THP-1 Macrophage Cytokine Multiplex................................................................91

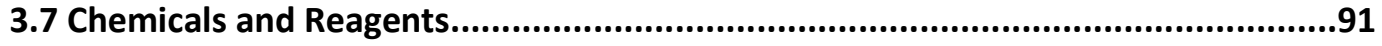

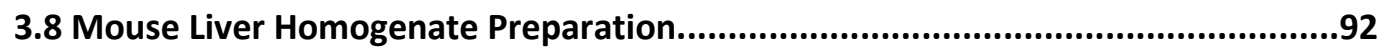

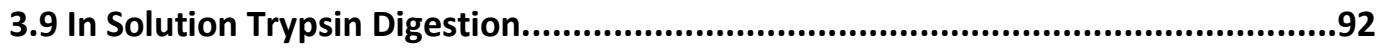

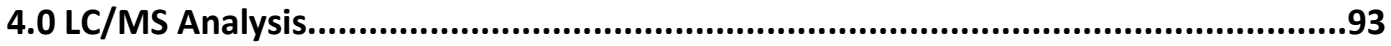

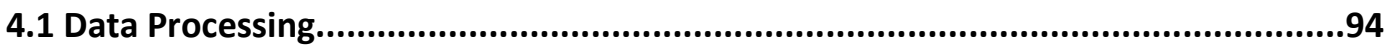

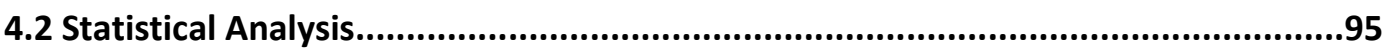

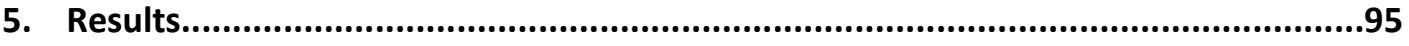

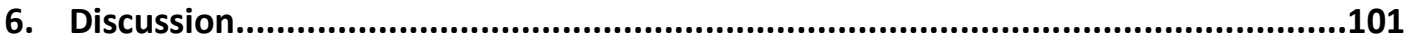

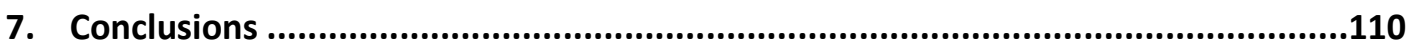

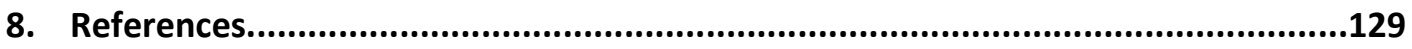




\section{CHAPTER 3: Neuroprotective effects of Pomegranate and Maple Syrup Extracts}

Manuscript 4: Pomegranate ellagitannin-gut microbial-derived metabolites, urolithins, inhibit neuroinflammation in vitro. DaSilva, N.A., Nahar, P.P., Ma, H., Eid, A., Wei, Z., Meschwitz, S., Zawia, N.H., Slitt, A.L. and Seeram, N.P., (2017) Nutritional neuroscience, pp.1-11.................. 136

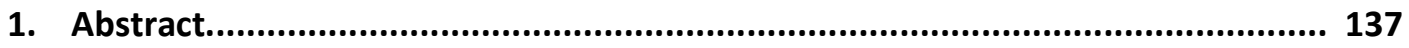

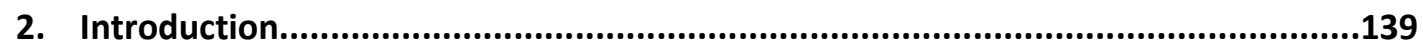

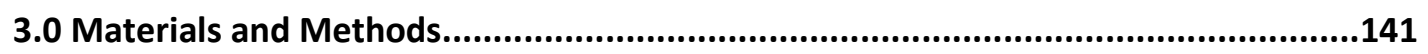

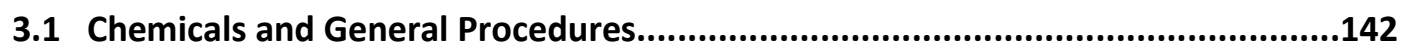

3.2 Alzheimer's Disease Transgenic Mouse Study.................................................142

3.3 RNA Extraction and qRT-PCR of Hippocampal Mouse Tissue............................142

3.4 Cell Culture

3.5 Cell Viability and Oxidative Stress Assays......................................................144

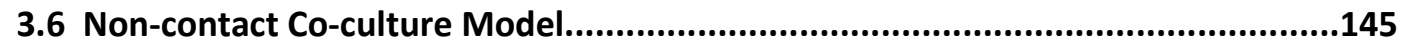

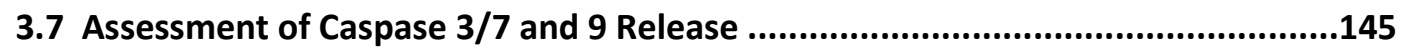

3.8 Measurement of Production of Nitric Oxide (NO).............................................146

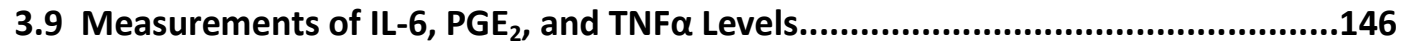

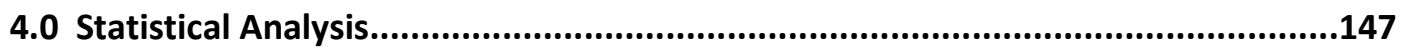

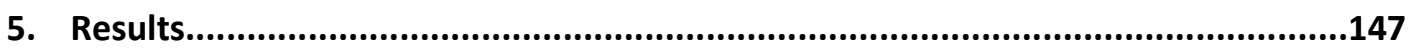

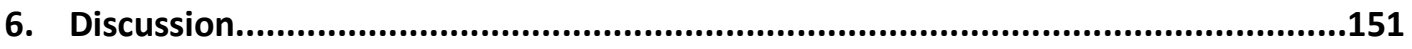

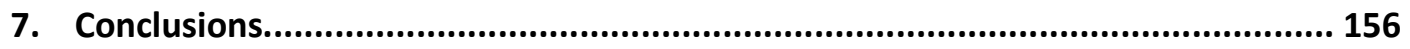

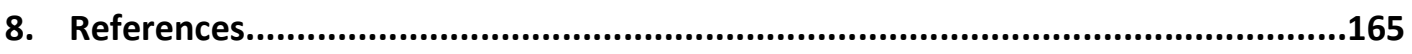


Manuscript 5: Effects of a Standardized Phenolic-Enriched Maple Syrup Extract on $\beta$-Amyloid Aggregation, Neuroinflammation in Microglial and Neuronal Cells, and $\beta$-Amyloid Induced Neurotoxicity in Caenorhabditis elegans. Ma H, DaSilva NA, Liu W, Nahar PP, Wei Z, Liu Y, Pham PT, Crews R, Vattem DA, Slitt AL, Shaikh ZA, Seeram NP (2016) Neurochemical research, 41(11), 2836-2847.

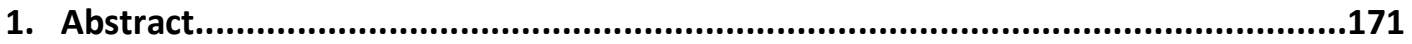

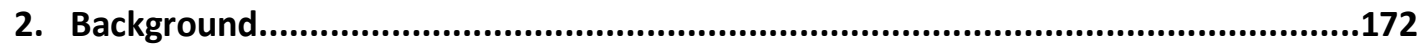

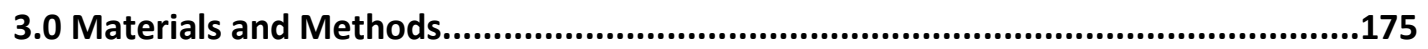

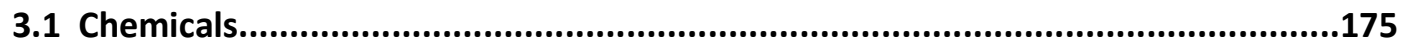

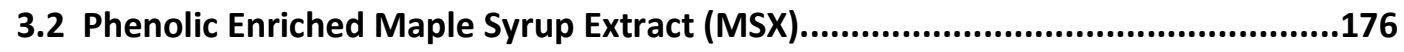

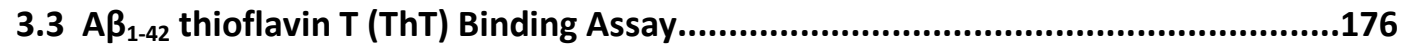

3.4 Transmission Electron Microscope (TEM) Analysis........................................177

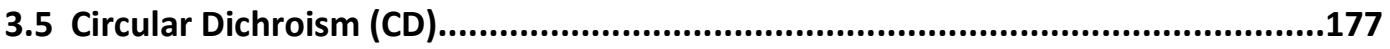

3.6 Dynamic Light Scattering (DLS) and Zeta Potential Measurement.......................178

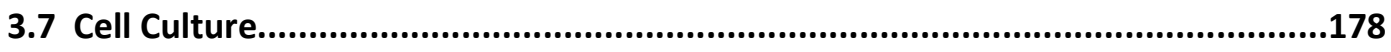

3.8 Measurement of Cell Viabilities.......................................................................179

3.9 Measurement of Levels of Reactive Oxygen Species (ROS)................................179

4.0 Measurement of Nitric Oxide Species Levels by Griess Assay............................180

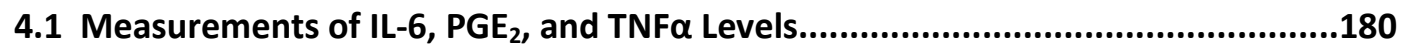

4.2 Non-contact Co-culture Model with BV-2 and SH-SY5Y Cells.............................180

4.3 Transgenic Caenorhabditis elegans in vivo Assay.............................................181

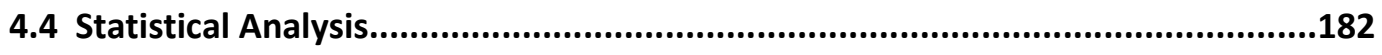

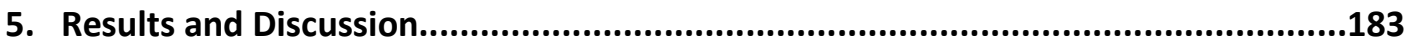

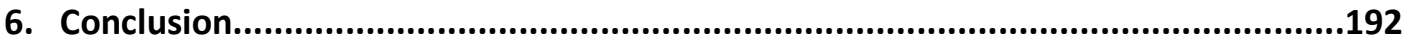

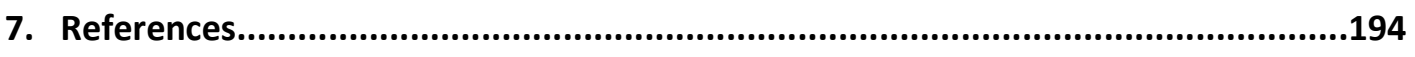

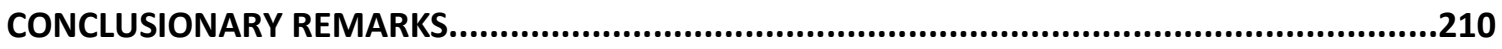




\section{Manuscript 1:}

Figure 1: High Fat Diet and High Fat Diet with PE show a general reduction in pro-inflammatory Interleukins.

Figure 2: Pro-inflammatory effects of High Fat Diet consumption are modulated with the inclusion of PE in feed....

Figure 3: High Fat Diet Fed Mice show increased levels of Alzheimer's Disease

related genes and compensatory mechanism associated anti-oxidant response genes

Figure 4: Macrophage Infiltration, Metabolic Syndrome associated ligand and receptor genes are differentially expressed with and without PE inclusion

Supplemental Figure 1: High Fat and Low Fat Diet Effects on Body, White Adipose, and Liver Weight with and without PE supplementation.

Supplemental Figure 2: Hepatic Triacylglycerides, Total Lipids, and Non-esterified Free Fatty Acids are abrogated with PE supplementation in both High Fat and Low Fat Diet fed mice....

\section{Manuscript 2:}

Figure 1: Effects of High Fat Diet and High Fat Diet with MSX show a general reduction in proinflammatory Interleukins

Figure 2: MSX modulates pro-inflammatory effects of High Fat Diet consumption

Figure 3: High Fat Diet Fed Mice show increased levels of Alzheimer's Disease related genes and compensatory mechanism associated anti-oxidant response genes

Figure 4: MSX modulates gene expression of Macrophage and Metabolic Syndrome associated ligands and receptor genes.

Supplemental Figure 1: Final Body and Tissue Weights after 12 Weeks of High Fat Diet exposure with or without MSX supplementation.

Supplemental Figure 2: Hepatic Lipid Composition of High Fat Diet fed mice with and without MSX

Supplemental Figure 3: Serum IL-6 concentrations are abrogated with the addition of MSX in Low Fat and High Fat Diets. 


\section{LIST OF FIGURES}

\section{Manuscript 3:}

Figure 1: Week 12 Glucose Tolerance Test (GTT) shows greater insensitivity to Glucose in HFD mice as compared to LFD mice.

Figure 2: Week 15 Insulin Tolerance Test (ITT) shows greater insensitivity to Insulin in HFD mice as compared to LFD mice.

Figure 3: Week 18 Necropsy Body Weight, Liver, and White Adipose Tissues Weights

Figure 4: Whole Liver comparisons between Low Fat Diet mice and High Fat diet mice with and without MSX supplementation

Figure 5: Serum concentrations of pro-inflammatory cytokine IL-6 are elevated in ALIOS mice but abrogated by MSX at Week 18.

Figure 6: Proteomic Analysis of Week 18 Livers reveal significant increases in NASH/NAFLD associated proteins including markers of fibrosis, apoptosis, and lipid regulation.

Figure 7: Volcano Plot of High Fat Diet and Low Fat Diet Liver Proteins 120

Figure 8: Volcano Plot of High Fat Diet and HFD+MSX0.5\% Liver Proteins 121

Figure 9: Volcano Plot of High Fat Diet and HFD+MSX0.05\%Liver Proteins .122

Figure 10: Volcano Plot of Low Fat Diet and LFD+MSX0.5\% Liver Proteins 123

Figure 11: Volcano Plot of Low Fat Diet and LFD+MSX0.05\% Liver Proteins

Figure 12: MSX mitigates initial LPS-induced inflammatory cytokine release in healthy human donor monocytes after 24 hours

Figure 13: MSX abrogates LPS-induced inflammatory cytokine release in differentiated THP-1 monocytes after 6 hrs

Figure 14: Low doses of MSX abrogates LPS-induced inflammatory cytokine release in differentiated THP-1 monocytes after 6 hrs

Supplemental Figure 1: Average Body Weights and Food Intake of Each Cage for 18 Weeks of Diet Administration. 


\section{Manuscript 4:}

Figure 1: Urolithins attenuate LPS-stimulated elevation of media nitric oxide (NO) in murine microglial cells 158

Figure 2: Urolithins modulate the release of pro-inflammatory cytokines in LPS stimulated murine BV-2 microglia..

Figure 3: Urolithins maintain human neuronal SH-SY5Y cell viability when exposed to LPS conditioned media from microglia.

Figure 4: Urolithins maintain BV-2 murine microglia and SH-SY5Y human neuron cell viability.

Figure 5: Hydrogen peroxide induced caspase 9 protease activity is decreased in murine microglia (BV-2) and differentiated human neuroblastoma.....

Figure 6: Hydrogen peroxide induced caspase $3 / 7$ release is abrogated in murine microglia (BV-2) and human neurons.

Figure 7: Pomegranate extract (PE) mitigates release of pro-inflammatory cytokines in hippocampi of transgenic mice fed PE.

\section{Manuscript 5:}

Figure 1: HPLC-DAD chromatogram of a phenolic-enriched maple syrup extract (MSX) showing 37 compounds identified.

Figure 2: ThT binding assay (A) and TEM analysis (B) showing the levels of $A \beta_{1-42}$ aggregation treated with different concentrations of MSX $(50-500 \mu \mathrm{g} / \mathrm{mL})$ or positive control resveratrol..202

Figure 3: Circular Dichroism (CD) measurement of native $A \beta$, aggregated $A \beta$, and $A \beta$ co-treated with MSX (500 ug/mL). 203

Figure 4: $\mathrm{MSX}$ reduced $\mathrm{H}_{2} \mathrm{O}_{2}$-induced oxidative stress in BV-2 murine microglial cells.............204

Figure 5: BV-2 LPS stimulated Cytokine Analysis (ELISA) with and without MSX...................... 205

Figure 6: MSX reduced murine BV-2 microglia-mediated neurotoxicity of human SH-SY5Y neuron cells 206

Figure 7: Mobility curves of transgenic (CL4176) C. elegans $20 \mathrm{~h}$ post $A \beta_{1-42}$ induction with and without MSX. 


\section{Manuscript 1}

Table 1: Multiplex Genes, Function, and Treatment Comparisons........................................... 36

\section{Manuscript 2:}

Table 1: Gene Multiplex Targets, Functions, and Statistical Comparisons. .70

\section{Manuscript 3:}

Table 1: Week 18 Histology Scoring Frequencies.

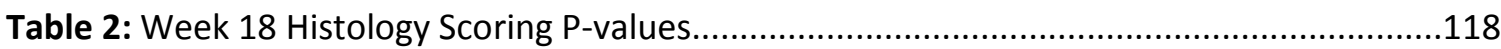

\section{Manuscript 4:}

None

\section{Manuscript 5:}

Table 1: Measurement of zeta potential, particle size distribution, and $\beta$-sheet content of native $A \beta_{1-42}$, aggregated $A \beta_{1-42}$ and $M S X(500 \mu \mathrm{g} / \mathrm{mL})$ treated $A \beta_{1-42}$ .208

Table 2: Survival (mean, median, and maximum) of (CL4176) C. elegans worms treated with MSX $(10 \mu \mathrm{g} / \mathrm{mL})$ post $A \beta_{1-42}$ induction of muscular paralysis at $25^{\circ} \mathrm{C}$ 


\section{JUSTIFICATION OF RESEARCH}

To date, chronic inflammation during the course of an individual's lifetime is cited as a significant causality to a number of diseases and disorders. Such diseases include diabetes, heart disease, liver disease, neurological diseases and even neoplasias of the liver, prostate, brain (Glioma/Glioblastoma) and colon ${ }^{1-4}$. Moreover, this inflammation stems from not just lifestyle choices (poor eating habits, smoking, alcohol consumption etc.) but from environmental toxicants (e.g. pollutants, pesticides, contaminants, radiation, pathogens etc.). Latter factors lead to the generation of free radicals and cause the malfunction of key cellular process proteins which are involved in cell repair and survival ${ }^{5,6}$.

Inflammation localized to the brain however, has been extensively studied and positively linked to the pathogenesis of Alzheimer's disease, Parkinson's disease, Amyotrophic Lateral Sclerosis, and Gliomas ${ }^{2,7-10}$. Neuroinflammation is a primary driver of Alzheimer's disease $^{11-13}$. One major hypothesis involves an axis between microglia (the resident macrophages of the brain) and neurons. The ability to mitigate the effects of damage associated molecules (e.g. Beta Amyloid, Advanced Glycation End products (AGEs), and ATP ) and pathogen associated molecules (e.g. Lipopolysaccharide(LPS), Flagellin, CpG Bacterial DNA, and Viral Envelope Particles) are regulated by microglia ${ }^{13,14}$.

Macrophages and microglia are able to recognize these molecules by way of Toll-Like receptors (TLRs) and by Nod-Like Receptors (NLRs). Toll-Like receptors are multi-subunit transmembrane proteins and will respond to specific pathogenic or damage molecule patterns by binding to one of the aforementioned ligands and create an intracellular signal triggering an intracellular cascade (increasing mitochondrial respiration, elevating arginase expression etc.) causing the microglial cell to exhibit a pro-inflammatory phenotype ${ }^{15}$. 
Microglia mediated neuronal cell death is the result of an over stimulation of microglia (aka reactive microgliosis) which will produce a multitude of pro-inflammatory stimuli including but not limited to, reactive metabolites such as Nitric Oxide (NO), Reactive Oxide Species (ROS) Prostaglandins (i.e. PGE 2 ), Th1 Cytokines (Tumor Necrosis Factor Alpha (TNF $\alpha$ ) and Interferon Gamma (IFNY)) , Interleukins (IL-1ß, IL-6, IL-8, etc.) and other pro-inflammatory molecules ${ }^{16-}$ ${ }^{18}$.These pro-inflammatory molecules signal to neighboring macrophages to be in an aggressive and consequently pro-inflammatory state thus perpetuating the secretion of inflammatory signaling molecules.

The constant activation of microglia is a major cause of both the hyperphosphorylation of microtubule associated protein tau (MAPT) and the cleavage of APP $\beta$ to neuro-toxic Beta Amyloid ${ }^{19,20}$. This finding supports that $A D$ is a progressive, multimodal disease state that includes an induction of $A \beta$ by inflammatory means, peptide deposition within the brain (which occurs 10-20 years before symptoms are presented), an increase in reactive species/inflammatory mediators, neuron cell death and a down-regulation of key $A \beta$ binding clearance proteins on macrophages and the epithelia on the Blood Brain Barrier (BBB) ${ }^{18,21-23}$.

Diet-induced obesity caused by sedentary behavior and poor diet, have been associated with metabolic syndromes such as type II diabetes mellitus (T2DM), nonalcoholic fatty liver disease (NAFLD) and even nonalcoholic steatohepatitis (NASH) ${ }^{24-27}$. The western diet is characterized to be high in saturated fats and sugars (sucrose/fructose) and has been shown in a number of mammalian studies to cause a dysbiosis of gut microbes ${ }^{28,29}$. 
This dysbiosis shifts the secondary metabolism of these microbes to produce harmful pro-inflammatory metabolites. These inflammatory metabolites not only alter localized innate immune response within the gut, but alter the integrity of the gut leading to what is regarded as "leaky-gut syndrome" ${ }^{30}$. The act of the gut lining being compromised, allows for these harmful metabolites and endotoxins (lipopolysaccharides (LPS)) to permeate through the large intestine into blood stream. This excess of pro-inflammatory stimuli causes a low-grade constant activation and differentiation of peripheral monocytes and macrophages. Therefore, this persistent release not only causes tissue damage within the gut, liver and cardiovascular system but also reduces sensitivity to insulin (and subsequently the tolerance to glucose), increases formation of sclerotic plaques within blood vessels, initiates fibrosis and cirrhosis in the liver and even alters the functionality of adipocytes leading to dyslipidemia (e.g. hyperlipidemia) ${ }^{31,32}$.

By modulating inflammation and the upstream targets to these diseases, this may serve as an alternative to simply "treating" to alleviate symptoms and only delay inevitable and irreversible patient outcomes. Therefore, prevention of these diseases by lowering inflammation and disease associated enzymes/proteins with a cost effective, low toxicity, and effective drug or chemical entities continues to challenge the pharmaceutical industry and academia $^{33-39}$. 
One potential source of these chemical entities could come from our diet in the form of gut microbe metabolites (GMMs). More specifically, metabolites derived from foods rich in polyphenols, such as ellagitannins, lignans, phytoestrogens, flavonoids, and phenolic acids ${ }^{40}$. Numerous studies have already shown the importance of the metabolome and their role in modulating several disease states ${ }^{41-44}$. In other words, the metabolism and otherwise biotransformation of large parent compounds by commensal bacteria into biologically active small molecule constituents has a profound impact on the maintenance of the host organism's overall health.

These metabolites are generated due to the diverse populations of bacteria that are harbored within the gut. Of these metabolites, is a group of small, rigid, planar compounds classified as urolithins ${ }^{45,46}$. Urolithins are the metabolites derived from gut microflora metabolism of ellagitannins, which are primarily sourced from oak aged beverages (e.g. wine, port, sherry, or whisky), raspberries, blackberries, walnuts, and pomegranate ${ }^{47-49}$.

Over the years, urolithins have gained appreciable amount of attention due to their small molecular weight (passive absorption into cells and other barriers), generally low toxicity systemically, and diverse functions as an anti-cancer and anti-inflammatory agent ${ }^{46,50-52}$. Furthermore, the biogeneration of urolithins are variable from individual to individual. The bacterial species responsible for the majority of this biotransformation is Gordonibacter urolithinfaciens and its activity/rate of biogenesis is dependent on the bacterial host's diet, body type, gender and age ${ }^{48,53-55}$. 
The other potential source of GMMs is from maple syrup, and more specifically, a proprietary polyphenol-enriched, food grade extract designated as MSX. Maple syrup is a widely used and accessible sweetener derived from the boiling of sap from Acer saccharum L. (Sugar Maple $)^{56}$. Recently, a food grade, polyphenol-enriched extract has been developed from polyphenol-rich fractions of maple syrup. This extract has been studied for its modulation of glucose consumption in liver cells (HepG2), abrogation of LPS mediated inflammation in murine macrophages (RAW 264.7) and safety in rats at doses up to $1000 \mathrm{mg} / \mathrm{kg} / \mathrm{day}{ }^{57}$.

Other maple syrup extracts have been produced in other labs but MSX is unique in its composition and method of preparation ${ }^{58,59}$. MSX contains over 60 phytochemicals from several polyphenol classes such as phenolic acids, coumarins, lignans, and stilbenes. One such polyphenolic class, lignans ( e.g. Secoisolariciresinol-diglucoside (SDG)), exhibit antiinflammatory and anti-cancer activity ${ }^{60,61}$. These lignans, while also regarded as phytoestrogens based on their structural resemblance to $17 \beta$-Estradiol, are able to be transformed by gut microbes to enterolignans such as Secoisolariciresinol (SECO), Enterodiol (ED), and Enterolactone $(\mathrm{EL})^{62,63}$.These enterolignans, while also found in flaxseed (Linum usitatissimum L.) are able to pass the gut membrane and enter systemic circulation ${ }^{64,65}$. Interestingly, of the metabolites, EL (one of the most biologically active but least abundant metabolite) has been found to enter the brain ${ }^{66,67}$. Therefore, MSX could serve as a source of the parent molecules needed to potentiate effects of inflammation.

Herein, this body of work will highlight some of the methods used to obtain data to further guide this exploration of PE and MSX for their role in mitigating inflammation mediated neurodegeneration in vitro as well as their role in modifying outcomes associated with metabolic syndrome in vivo. 


\section{REFERENCES}

1. Barzilay, J. \& Freedland, E. Inflammation and its association with glucose disorders and cardiovascular disease. Treat. Endocrinol. 2, 85-94 (2003).

2. Kore, R. A. \& Abraham, E. C. Inflammatory cytokines, interleukin-1 beta and tumor necrosis factor-alpha, upregulated in glioblastoma multiforme, raise the levels of CRYAB in exosomes secreted by U373 glioma cells. Biochem. Biophys. Res. Commun. 453, 326-331 (2014).

3. Dmitrieva, O. S., Shilovskiy, I. P., Khaitov, M. R. \& Grivennikov, S. I. Interleukins 1 and 6 as Main Mediators of Inflammation and Cancer. Biochem. Biokhimiia 81, 80-90 (2016).

4. Duncan, B. B. et al. Low-grade systemic inflammation and the development of type 2 diabetes: the atherosclerosis risk in communities study. Diabetes 52, 1799-1805 (2003).

5. Dandona, P. Endothelium, inflammation, and diabetes. Curr. Diab. Rep. 2, 311-315 (2002).

6. Wright, C. \& Simone, N. L. Obesity and tumor growth: inflammation, immunity, and the role of a ketogenic diet. Curr. Opin. Clin. Nutr. Metab. Care 19, 294 (2016).

7. Morales, I., Guzmán-Martínez, L., Cerda-Troncoso, C., Farías, G. A. \& Maccioni, R. B. Neuroinflammation in the pathogenesis of Alzheimer's disease. A rational framework for the search of novel therapeutic approaches. Front. Cell. Neurosci. 8, 112 (2014).

8. Chen, W.-W., Zhang, X. \& Huang, W.-J. Role of neuroinflammation in neurodegenerative diseases (Review). Mol. Med. Rep. 13, 3391-3396 (2016).

9. Bahia El Idrissi, N. et al. Complement activation at the motor end-plates in amyotrophic lateral sclerosis. J. Neuroinflammation 13, 72 (2016).

10. Klein, A. et al. Astrocytes facilitate melanoma brain metastasis via secretion of IL-23. J. Pathol. 236, 116-127 (2015).

11. Koyama, A. et al. The role of peripheral inflammatory markers in dementia and Alzheimer's disease: a meta-analysis. J. Gerontol. A. Biol. Sci. Med. Sci. 68, 433-440 (2013).

12. Hung, A. S. M. et al. Mutated tau, amyloid and neuroinflammation in Alzheimer disease-A brief review. Prog. Histochem. Cytochem. 51, 1-8 (2016). 
13. Li, X.-H. et al. AGEs induce Alzheimer-like tau pathology and memory deficit via RAGEmediated GSK-3 activation. Neurobiol. Aging 33, 1400-1410 (2012).

14. Münch, G., Westcott, B., Menini, T. \& Gugliucci, A. Advanced glycation endproducts and their pathogenic roles in neurological disorders. Amino Acids 42, 1221-1236 (2012).

15. Landreth, G. E. \& Reed-Geaghan, E. G. Toll-like receptors in Alzheimer's disease. Curr. Top. Microbiol. Immunol. 336, 137-153 (2009).

16. Tuppo, E. E. \& Forman, L. J. Free radical oxidative damage and Alzheimer's disease. J. Am. Osteopath. Assoc. 101, S11-15 (2001).

17. Baranello, R. J. et al. Amyloid-beta protein clearance and degradation ( $A B C D)$ pathways and their role in Alzheimer's disease. Curr. Alzheimer Res. 12, 32-46 (2015).

18. Dudvarski Stankovic, N., Teodorczyk, M., Ploen, R., Zipp, F. \& Schmidt, M. H. H. Microgliablood vessel interactions: a double-edged sword in brain pathologies. Acta Neuropathol. (Berl.) 131, 347-363 (2016).

19. Doens, D. \& Fernández, P. L. Microglia receptors and their implications in the response to amyloid $\beta$ for Alzheimer's disease pathogenesis. J. Neuroinflammation 11, 48 (2014).

20. Hüll, M., Lieb, K. \& Fiebich, B. L. Pathways of inflammatory activation in Alzheimer's disease: potential targets for disease modifying drugs. Curr. Med. Chem. 9, 83-88 (2002).

21. Griffin, W. S. T., Liu, L., Li, Y., Mrak, R. E. \& Barger, S. W. Interleukin-1 mediates Alzheimer and Lewy body pathologies. J. Neuroinflammation 3, 5 (2006).

22. Ni, J., Wang, P., Zhang, J., Chen, W. \& Gu, L. Silencing of the $P 2 X(7)$ receptor enhances amyloid- $\beta$ phagocytosis by microglia. Biochem. Biophys. Res. Commun. 434, 363-369 (2013).

23. Halle, A. et al. The NALP3 inflammasome is involved in the innate immune response to amyloid-beta. Nat. Immunol. 9, 857-865 (2008).

24. Kusminski, C. M., Shetty, S., Orci, L., Unger, R. H. \& Scherer, P. E. Diabetes and apoptosis: lipotoxicity. Apoptosis Int. J. Program. Cell Death 14, 1484-1495 (2009). 
25. Cosentino, F. \& Assenza, G. E. Diabetes and Inflammation. Herz 29, 749-759 (2004).

26. Panasevich, M. R., Peppler, W. T., Oerther, D. B., Wright, D. C. \& Rector, R. S. Microbiome and NAFLD: potential influence of aerobic fitness and lifestyle modification. Physiol. Genomics 49, 385-399 (2017).

27. Dongiovanni, P. \& Valenti, L. A Nutrigenomic Approach to Non-Alcoholic Fatty Liver Disease. Int. J. Mol. Sci. 18, (2017).

28. Panasevich, M. R. et al. High-fat, high-fructose, high-cholesterol feeding causes severe NASH and cecal microbiota dysbiosis in juvenile Ossabaw swine. Am. J. Physiol. Endocrinol. Metab. 314, E78-E92 (2018).

29. Pierantonelli, I. et al. Lack of NLRP3-inflammasome leads to gut-liver axis derangement, gut dysbiosis and a worsened phenotype in a mouse model of NAFLD. Sci. Rep. 7, 12200 (2017).

30. Fändriks, L. Roles of the gut in the metabolic syndrome: an overview. J. Intern. Med. 281, 319-336 (2017).

31. Ilan, Y. Leaky gut and the liver: a role for bacterial translocation in nonalcoholic steatohepatitis. World J. Gastroenterol. 18, 2609-2618 (2012).

32. Lim, H.-W. \& Bernstein, D. E. Risk Factors for the Development of Nonalcoholic Fatty Liver Disease/Nonalcoholic Steatohepatitis, Including Genetics. Clin. Liver Dis. 22, 39-57 (2018).

33. Norton, S., Matthews, F. E., Barnes, D. E., Yaffe, K. \& Brayne, C. Potential for primary prevention of Alzheimer's disease: an analysis of population-based data. Lancet Neurol. 13, 788-794 (2014).

34. Panza, F. et al. Beyond the neurotransmitter-focused approach in treating Alzheimer's disease: drugs targeting beta-amyloid and tau protein. Aging Clin. Exp. Res. 21, 386-406 (2009).

35. Saito, S. \& Ihara, M. New therapeutic approaches for Alzheimer's disease and cerebral amyloid angiopathy. Front. Aging Neurosci. 6, 290 (2014). 
36. Venigalla, M., Sonego, S., Gyengesi, E., Sharman, M. J. \& Münch, G. Novel promising therapeutics against chronic neuroinflammation and neurodegeneration in Alzheimer's disease. Neurochem. Int. 95, 63-74 (2016).

37. Salomone, F., Godos, J. \& Zelber-Sagi, S. Natural antioxidants for non-alcoholic fatty liver disease: molecular targets and clinical perspectives. Liver Int. Off. J. Int. Assoc. Study Liver 36, 5-20 (2016).

38. Rigano, D., Sirignano, C. \& Taglialatela-Scafati, O. The potential of natural products for targeting PPAR $\alpha$. Acta Pharm. Sin. B 7, 427-438 (2017).

39. Sharma, H. \& Kumar, S. Natural AMPK Activators: An Alternative Approach for the Treatment and Management of Metabolic Syndrome. Curr. Med. Chem. 24, 1007-1047 (2017).

40. Libro, R., Giacoppo, S., Soundara Rajan, T., Bramanti, P. \& Mazzon, E. Natural Phytochemicals in the Treatment and Prevention of Dementia: An Overview. Mol. Basel Switz. 21, 518 (2016).

41. Shore, S. A. \& Cho, Y. Obesity and Asthma: Microbiome-Metabolome Interactions. Am. J. Respir. Cell Mol. Biol. 54, 609-617 (2016).

42. Kelly, D. L., Lyon, D. E., Yoon, S. L. \& Horgas, A. L. The Microbiome and Cancer: Implications for Oncology Nursing Science. Cancer Nurs. 39, E56-62 (2016).

43. Fröhlich, E. E. et al. Cognitive impairment by antibiotic-induced gut dysbiosis: Analysis of gut microbiota-brain communication. Brain. Behav. Immun. 56, 140-155 (2016).

44. Wong, M.-L. et al. Inflammasome signaling affects anxiety- and depressive-like behavior and gut microbiome composition. Mol. Psychiatry 21, 797-805 (2016).

45. Tomás-Barberán, F. A. et al. Urolithins, the rescue of 'old' metabolites to understand a 'new' concept: Metabotypes as a nexus among phenolic metabolism, microbiota dysbiosis, and host health status. Mol. Nutr. Food Res. 61, (2017).

46. Espín, J. C., Larrosa, M., García-Conesa, M. T. \& Tomás-Barberán, F. Biological significance of urolithins, the gut microbial ellagic Acid-derived metabolites: the evidence so far. Evid.Based Complement. Altern. Med. ECAM 2013, 270418 (2013). 
47. Sánchez-González, C., Ciudad, C. J., Noé, V. \& Izquierdo-Pulido, M. Health benefits of walnut polyphenols: An exploration beyond their lipid profile. Crit. Rev. Food Sci. Nutr. 57, 33733383 (2017).

48. Tomás-Barberán, F. A., García-Villalba, R., González-Sarrías, A., Selma, M. V. \& Espín, J. C. Ellagic acid metabolism by human gut microbiota: consistent observation of three urolithin phenotypes in intervention trials, independent of food source, age, and health status. J. Agric. Food Chem. 62, 6535-6538 (2014).

49. Garcia-Muñoz, C. \& Vaillant, F. Metabolic fate of ellagitannins: implications for health, and research perspectives for innovative functional foods. Crit. Rev. Food Sci. Nutr. 54, 15841598 (2014).

50. Yuan, T. et al. Pomegranate's Neuroprotective Effects against Alzheimer's disease Are Mediated by Urolithins, Its Ellagitannin-Gut Microbial Derived Metabolites. ACS Chem. Neurosci. 7, 26-33 (2016).

51. Sharma, M. et al. Effects of fruit ellagitannin extracts, ellagic acid, and their colonic metabolite, urolithin A, on Wnt signaling. J. Agric. Food Chem. 58, 3965-3969 (2010).

52. Piwowarski, J. P., Kiss, A. K., Granica, S. \& Moeslinger, T. Urolithins, gut microbiota-derived metabolites of ellagitannins, inhibit LPS-induced inflammation in RAW 264.7 murine macrophages. Mol. Nutr. Food Res. 59, 2168-2177 (2015).

53. Selma, M. V. et al. The human gut microbial ecology associated with overweight and obesity determines ellagic acid metabolism. Food Funct. 7, 1769-1774 (2016).

54. Selma, M. V., Tomás-Barberán, F. A., Beltrán, D., García-Villalba, R. \& Espín, J. C. Gordonibacter urolithinfaciens sp. nov., a urolithin-producing bacterium isolated from the human gut. Int. J. Syst. Evol. Microbiol. 64, 2346-2352 (2014).

55. Selma, M. V., Beltrán, D., García-Villalba, R., Espín, J. C. \& Tomás-Barberán, F. A. Description of urolithin production capacity from ellagic acid of two human intestinal Gordonibacter species. Food Funct. 5, 1779-1784 (2014).

56. Perkins, T. D. \& van den Berg, A. K. Maple syrup-production, composition, chemistry, and sensory characteristics. Adv. Food Nutr. Res. 56, 101-143 (2009). 
57. Zhang, Y. et al. Chemical compositional, biological, and safety studies of a novel maple syrup derived extract for nutraceutical applications. J. Agric. Food Chem. 62, 6687-6698 (2014).

58. Kamei, A. et al. Administration of a maple syrup extract to mitigate their hepatic inflammation induced by a high-fat diet: a transcriptome analysis. Biosci. Biotechnol. Biochem. 79, 1893-1897 (2015).

59. Hawco, C. L. A., Wang, Y., Taylor, M. \& Weaver, D. F. A Maple Syrup Extract Prevents $\beta$ Amyloid Aggregation. Can. J. Neurol. Sci. J. Can. Sci. Neurol. 43, 198-201 (2016).

60. Prasad, null. Antioxidant Activity of Secoisolariciresinol Diglucoside-derived Metabolites, Secoisolariciresinol, Enterodiol, and Enterolactone. Int. J. Angiol. Off. Publ. Int. Coll. Angiol. Inc 9, 220-225 (2000).

61. Di, Y., De Silva, F., Krol, E. S. \& Alcorn, J. Flaxseed Lignans Enhance the Cytotoxicity of Chemotherapeutic Agents against Breast Cancer Cell Lines MDA-MB-231 and SKBR3. Nutr. Cancer 70, 306-315 (2018).

62. Belcher, S. M. \& Zsarnovszky, A. Estrogenic actions in the brain: estrogen, phytoestrogens, and rapid intracellular signaling mechanisms. J. Pharmacol. Exp. Ther. 299, 408-414 (2001).

63. Aura, A.-M. Microbial metabolism of dietary phenolic compounds in the colon. Phytochem. Rev. 7, 407-429 (2008).

64. Mukker, J. K., Singh, R. S. P., Muir, A. D., Krol, E. S. \& Alcorn, J. Comparative pharmacokinetics of purified flaxseed and associated mammalian lignans in male Wistar rats. Br. J. Nutr. 113, 749-757 (2015).

65. Quartieri, A. et al. Detection of novel metabolites of flaxseed lignans in vitro and in vivo. Mol. Nutr. Food Res. 60, 1590-1601 (2016).

66. Jan, K.-C., Hwang, L. S. \& Ho, C.-T. Tissue distribution and elimination of sesaminol triglucoside and its metabolites in rat. Mol. Nutr. Food Res. 53, 815-825 (2009).

67. Saarinen, N. M. \& Thompson, L. U. Prolonged administration of secoisolariciresinol diglycoside increases lignan excretion and alters lignan tissue distribution in adult male and female rats. Br. J. Nutr. 104, 833-841 (2010). 


\section{MANUSCRIPT 1}

\section{In preparation for submission to Nutritional Metabolism}

Anti-inflammatory and neuroprotective effects of a standardized pomegranate extract in dietinduced obese C57BL/6 mice.

Nicholas A. DaSilva ${ }^{1}$, Marisa Pfohl ${ }^{1}$, Emily Marques ${ }^{1}$, Hang Ma ${ }^{1}$, Juliana Agudelo ${ }^{1}$, Angela L. Slitt $^{1,2}$, Navindra P. Seeram ${ }^{1,2}$

Affiliations:

${ }^{1}$ Department of Biomedical and Pharmaceutical Sciences, University of Rhode Island, Kingston, RI USA

${ }^{2}$ George and Anne Ryan Institute for Neuroscience, University of Rhode Island, Kingston, RI USA Corresponding Authors:

Navindra Seeram, Ph.D.; University of Rhode Island, 495B, Avedisian Hall, 7 Greenhouse Rd. Kingston, RI, 02881, USA, Phone: 401-874-9367, Email: nseeram@uri.edu Angela L. Slitt, Ph.D.; University of Rhode Island, 395D, Avedisian Hall, 7 Greenhouse Rd. Kingston, RI 02881, USA, Phone: 401-874-5020, Email: aslitt@uri.edu 


\section{Abstract}

Background: Poor diet can promote obesity and gut microbe dysbiosis, which increases circulating endotoxin levels. In the brain, this inflammatory stimulus activates and promotes recruitment of microglia leading to reactive microgliosis and implicated in the pathogenesis of a number of neurodegenerative diseases. Pomegranate extract (PE) could be a potential source of beneficial phytonutrients capable of mitigating these inflammatory processes from occurring. Methods: Male C57BL/6 mice fed either a high fat diet (45\% kcal from fat) or a standard grain free rodent diet ( $10 \% \mathrm{kcal}$ from fat) with or without PE at a dose of $1 \% \mathrm{w} / \mathrm{w}$ for 12 weeks producing an a diet-induced obesity phenotype. Mice were sacrificed following 12 weeks and whole hippocampi were excised for multiplexed gene expression analysis of inflammatory and neurodegenerative disease associated genes.

Results: Mice fed a HFD for 12 weeks exhibited elevated expression of both pro- and antiinflammatory cytokine/growth factors and neurodegenerative associated genes in the hippocampus. In HFD mice, PE supplementation allowed for a significant reduction in the gene expression of a number of key pro-inflammatory cytokines such as IL-1 $\alpha$, IL-1 $\beta$, IL-7, IL-11, TNF $\alpha$, MAPT, APP, GSK3 $\beta$, and LEPR.

Conclusions: In the hippocampus, immune cell recruitment and differentiation for tissue repair, leptin signaling and neurofibril processing via the MAPT-GSK3 $\beta$-APP axis are significantly modulated by PE in a diet-induced obesity murine model. 


\section{Background}

Poor diet has been linked to the generation of low grade inflammation systemically ${ }^{1}$. Dietary factors also influence the onset of Type II Diabetes Mellitus (T2DM), Cardiovascular Disease, Non Alcoholic Steatohepatitis (NASH), Non Alcoholic Fatty Liver Disease (NAFLD) and numerous forms of neoplasias ${ }^{2}$. Gut microbes are tasked with not only aiding in nutrient extraction, immune cell programming, and maintaining intestinal barrier integrity ${ }^{3}$. Specific pathogenic strains may be promoted through poor diet and thus are a major source of proinflammatory molecules such as lipopolysaccharide (LPS) $)^{4,5}$. LPS, a pathogen associated molecular pattern (PAMP), is recognized by Toll Like Receptors of macrophages which signal for recruitment and activation of additional lymphocytes and leukocytes ${ }^{6,7}$. LPS has been found in the plasma of both obese individuals and in patients diagnosed with neurodegenerative diseases (e.g. Alzheimer's disease (AD), Parkinson's disease (PD) and Amyotrophic Lateral Sclerosis $(A L S))^{8-10}$. These diseases represent a significant portion of the global economic burden associated with an aging population ${ }^{11}$. The progressive loss in neuron function and viability is associated with aging and neuroinflammation ${ }^{12}$. Neuroinflammation, which is largely governed by the resident immune cells of the brain, microglia ${ }^{13}$ has been associated with the etiology of $A D$ and $P D$ in rodents and patients ${ }^{14,15}$.

One commonly observed site of this neuroinflammatory process is the hippocampus.

The hippocampus is responsible for maintaining basic cognitive function and memory and is one of the first regions to be affected by this inflammatory insult ${ }^{16,17}$. The hippocampus is also a major site for the accumulation of neurotoxic neurofibrillary plaques and tangles ${ }^{18,19}$. 
By feeding mice a diet rich in saturated fats, this diet promotes features exemplified by obese individuals consuming a "western" diet as seen in North America and Europe ${ }^{20}$.

Treatments for these diet associated disorders range from lipid/cholesterol reducers Statins (atorvastatin, simvastatin), insulin sensitizers such as metformin and thiazolidinediones (e.g. rosiglitazone), alpha-glucosidase inhibitors, Fibrates, Non-steroidal Anti Inflammatory Drugs (NSAIDs) and cholinesterase Inhibitors ${ }^{21-27}$. These therapies, which are linked to a number of undesirable side effects, have been substituted for less detrimental forms of medicine such as diet restrictions, bariatric surgery, and exercise $\mathrm{e}^{21,22}$. Natural products have also been considered for patients with metabolic syndrome (MetS) on the basis of safety and cost ${ }^{28-30}$. While numerous in vitro, in vivo and human clinical studies with natural product usage in MetS diseases such as NASH have been published, understanding of the underlying molecular mechanisms of natural products and traditional herbal medicines (e.g. Ayurveda and Traditional Chinese medicine) in the brain are less defined. Polyphenols, represent a significant portion of the natural products commonly studied in age-related disease states and cancer ${ }^{31-34}$.

One source of these natural products is derived from pomegranate (Punica granatum). A commercially available pomegranate extract (PE) which is standardized to its major chemical constituent, Punicalagin (PA), has also been studied for its anti-bacterial, anti-inflammatory and anti-diabetic properties ${ }^{35-38}$. PA and its metabolite, ellagic acid (EA), are known to be metabolized by specific gut microbes and transformed to a class of significantly studied and therapeutically valuable compounds known as urolithins ${ }^{39,40}$. 
The anti-inflammatory capacity and mechanisms of these constituents have also been investigated using an in vitro model of neurodegeneration with murine microglia and human neurons ${ }^{41,42}$. Our group has previously measured the ability of PE to combat the effects of $A D$ in an Beta-amyloid transgenic mouse model ${ }^{43}$. Herein, we sought to use PE to modulate the proinflammatory effects of a western style diet specifically in the brains of wild type C57BL/6 mice.

\section{Materials and Methods}

\section{Animals and study conditions}

C57BL/6 mice were acquired from Jackson Labs (Bar Harbor, ME USA), acclimated for at least two weeks before being weight paired and housed four mice per cage. Mice were housed under 12 hour light/dark cycles and were allowed to consume food and water ad libitum. Mice were fed either a standard grain- free low fat diet (LFD) ( $n=8)$ (Research Diets Cat\# D12450B, New Brunswick, NJ), or a high fat diet where $45 \%$ of calories are derived from fat (HFD) ( $n=8$ ) (Research Diets Cat\# D12451, New Brunswick, NJ). PE was provided by Verdure Sciences (Noblesville, IN, USA) and was incorporated directly into LFD $(n=8)$ or HFD $(n=8)(1 \% w / w)$. Body weights and food consumption were monitored weekly. Following 12 weeks of diet administration, all mice were anesthetized using isoflurane and sacrificed by cervical dislocation. Tissues were immediately harvested and snap frozen in liquid nitrogen. Brains were separated into cortex, cerebellum, and hippocampus regions for future analysis. This study and its protocols were approved by and conducted in accordance with the University of Rhode Island Institutional Animal Care and Use Committee (IACUC) (Approval \# AN09-07-004). 


\section{Hepatic Lipid Isolation and Analysis}

Liver lipids from approximately $50 \mathrm{mg}$ of liver tissue were prepared using a chloroformmethanol extraction method described by More et al ${ }^{44}$. Triacylglyceride (TAG), Total Cholesterol, and Total Lipids were measured using specified kits from Wako Chemicals (Richmond VA USA).Total Non-esterified Free Fatty Acids (NEFA) were quantified using a kit from Pointe Scientific (Ann Arbor, MI USA).

\section{Nucleic Acid Isolation from Tissue}

Whole hippocampi were isolated at the time of necropsy and snap frozen for nucleic acid isolation. Tissue was subsequently homogenized using Trizol and a dounce homogenizer. Homogenized material was then added directly to a Trizol Spin Column (Zymo Research, San Diego, CA USA) to isolate nucleic acids according to the manufacturer's protocol. RNA concentration and quality was then determined using a spectrophotometer (Nanodrop, Thermo Fisher, Waltham, MA USA)

\section{Gene Expression Analysis}

Genes involved in inflammatory, neurodegenerative, and antioxidant response processes were multiplexed from single RNA samples using a custom Quantigene panel (Thermo Fisher, Waltham, MA USA). Analysis of the multiplex panel was performed according to the manufacturer's protocols with mean fluorescence intensity quantified using a Bioplex 200 instrument (Bio-Rad Laboratories, Hercules, CA USA). Genes of interest include IL-1 $\alpha$, IL-1 $\beta$, IL-2, IL-3, IL-4, IL-6, IL-7, IL-9, IL-10, IL-11, IL-13, IL-15, IL-16, IL-18, IL-19, IFN $\alpha$, IFNץ, TNF, CD38, CD70, FASL, MIF, CSF1, CD40LG, LEPR, CD36, MAPT, GSK3ß, APP, PPARY, ITGAM, STING, NOS2, SOD1, and NFEL2. 


\section{Statistical Analysis}

All gene expression data shown as mean fluorescence intensity (MFI) \pm standard error (SEM), was analyzed using one way analysis of variance (ANOVA) where $p<0.05$ were statistically significant. Body weight, tissue weight, and hepatic lipid data are shown as mean \pm SEM was compared for statistical significance using ANOVA and fisher's exact test. Calculations were performed using Graphpad Prism (Graphpad Prism Software for Windows Ver 8.0, La Jolla, CA USA). 


\section{Results}

\section{PE supplementation attenuated HFD fed white adipose, liver and body weight gains}

Final body weights of HFD mice $(42.6 \mathrm{~g} \pm 1.70 \mathrm{~g})$ following 12 weeks of a high fat diet exposure were significantly increased as compared to LFD mice $30.9 \mathrm{~g} \pm 0.92 \mathrm{~g}$. Liver weights were also elevated when comparing LFD fed mice $(1.26 \mathrm{~g} \pm 0.16 \mathrm{~g})$ to HFD fed mice $(1.45 \mathrm{~g} \pm 0.14 \mathrm{~g})$. PE did not alter food consumption as compared to control LFD and HFD fed mice. White adipose tissue (WAT) was also increased in HFD mice by $112.6 \%$ as compared to LFD control mice

(Supplemental Figure 1). However, the incorporation of PE into HFD feed decreased body, liver, and WAT weights by approximately $21.8,30.4$ and $65.6 \%$ respectively compared to the HFD control group.

PE reduces hepatic triacylglyceride, total lipid, and non-esterified free fatty acid levels in both HFD and LFD Fed Mice

Given the previous observations, PE's effect on the liver and fatty acid metabolism/catabolism was assessed by measuring total liver lipids, non-esterified free fatty acids (NEFA), cholesterol and triacyglycerides (TAG). The HFD increased hepatic total lipid and TAG content when compared to LFD controls by approximately 41.1 and $56.1 \%$ respectively. PE inclusion in the high fat diet significantly reduced total lipid and TAG content by approximately 41.8 and $60.2 \%$ respectively as compared to HFD control mice (Supplemental Figure 2). NEFA levels were also elevated in HFD mice by week 12 by $33.4 \%$ as compared to Low Fat Diet Controls. Moreover, PE inclusion reduced NEFA by $72.5 \%$ as compared to HFD control mice and by $39.4 \%$ as compared to LFD control mice. 
PE attenuates the gene expression of key Interleukins and inflammatory mediators in HFD fed mice

HFD fed mice exhibited statistically significant increases in all interleukins $(1,2,3,4,6,7$, $9,10,11,13,15,16,18,19)$ and interferons ( $\alpha$ and $\gamma$ ) as compared to the LFD diet fed mice in Figures 1 and 2. Gene expression of IL-1 $\alpha$, IL-1 $\beta$, IL-7, IL-11, and TNF between HFD and HFD+PE mice, were significantly decreased by $35.3 \%, 37.5 \%, 40.1 \%, 41.1 \%$ and $41.1 \%$.respectively. Several other cytokines showed decreasing trends but were not statistically significant. Interestingly, IL-2, IL-3, and IL-13 were upregulated by $29.6 \%, 30.3 \%$ and $114.7 \%$ respectively when comparing HFD to HFD+PE. To limit dietary variables and further elucidate the beneficial mechanisms associated with PE consumption, a cohort of mice were administered a LFD+PE diet. Interestingly, in the LFD+PE group, IL-13, IL-2, IL-3, IL-13, and IL-19 were noted with significantly increased expression levels. 


\section{PE-supplementation differentially modulates gene expression of antioxidant enzymes, reactive species, and neurodegenerative disease associated proteins}

Mice consuming a high fat diet rich in saturated fats and sugars demonstrated the observation noted by other rodent studies involving accelerated ageing and poor diet. This HFD diet led to the elevation of genes commonly associated with Alzheimer's disease: Microtubule Associated Protein Tau (MAPT/Tau), Glycogen synthase kinase 3 Beta (GSK3ß), and Amyloid Precursor Protein (APP) by 1.33, 1.31 and 1.29 fold increase over LFD respectively (Figure 3). Each gene saw significant abrogation when comparing HFD+PE with HFD controls by $27.6 \%$, $24.2 \%$, and $19.4 \%$ respectively.

Nitric Oxide Synthase 2 (NOS2) and Super Oxide Dismutase 1 (SOD1) were significantly overexpressed in HFD mice compared to LFD fed mice (76.6\% and $39.7 \%$ respectively). PE supplemented LFD mice increased NOS2 gene expression by $43.4 \%$ as compared to the LFD control mice (Figure 3). Moreover, SOD1 and NOS2 were decreased by $14.9 \%$ and $1.9 \%$ as compared to the HFD control mice although these decreases were not statistically significant. Another major Antioxidant response gene, NFE2L2 (commonly known as NRF2), was significantly upregulated by $61.1 \%$ in HFD fed mice as compared to LFD controls. The addition to PE to HFD reduced this expression of NRF2 by $32.1 \%$ as compared to mice fed a HFD. PPAR $y$, ITGAM and STING were augmented in HFD fed mice as compared to their standard chow fed counterparts by $53.7 \%, 62.5 \%$, and $40.6 \%$ respectively. The addition of PE to either diet saw no statistically appreciable decrease when comparing gene expression to HFD controls. 


\section{PE addition to HFD modulates fatty acid transport and leptin receptor signaling genes}

The HFD increased CD38, CD70, FAS ligand, CD40 ligand by approximately $135.3 \%$, $288.4 \%, 184.7 \%$, and $88.6 \%$ respectively as compared to LFD control mice (Figure 4). Macrophage/Microglial associated genes, Macrophage Infiltration Factor (MIF) and Colony Stimulating Factor 1 (CSF1) were also significantly upregulated by $46.4 \%$ and $74.8 \%$ respectively in the HFD fed mice. PE supplementation to LFD increased the hippocampal gene expression of both MIF and CSF1 by $26.2 \%$ and $42.7 \%$ respectively. Interestingly, Leptin Receptor and CD36, two major Fatty Acid Transport associated genes, were also markedly increased in HFD fed mice by $192.1 \%$ and $82.8 \%$ respectively. PE supplementation in HFD reduced the gene expression of LEPR and CD36 by $41 \%$ and $23.7 \%$ in the hippocampus respectively. Moreover, HFD+PE mice decreased LEPR expression to levels similar to LFD control mice (7.13 MFI \pm 0.94$)$. 


\section{Discussion}

In the case of neurodegenerative diseases, studies have shown that specific dietary components may contribute to the weakening of the blood brain barrier and ultimately the

progressive activation of microglia ${ }^{20,45}$. Microglial activation and recruitment, is inherently a response to rid the tissue of foreign infection and/or damaged cells. Over time, the signaling capacity of microglia to respond to pathogen associated molecules (i.e. lipopolysaccharide or bacterial DNA) or damage associated molecules (i.e. nucleic acids from necrotic/apoptotic cells) weakens and thus to compensate, more macrophages are recruited further secreting inflammatory mediators ${ }^{12,13}$. This flood of inflammatory stimuli leads to the necrosis and apoptosis of neurons and depending on the localization of this degradation, manifests into $A D$, PD or $\mathrm{ALS}^{46}$.

Diet as a means of intervention in these initial inflammatory processes, are largely due in part to anti-oxidant activity of food sources. PE represents a therapeutically valuable source of chemical constituents. In this study, mice were subjected to a diet rich in saturated fats (45\%kcal derived from fat) for approximately 12 weeks. Body and Liver weights were significantly increased along with glucose and insulin tolerances as a result of this exposure to a HFD as compared to their standard chow controls (10\% kcal from fat). PE did not alter food consumption as compared to control LFD and HFD fed mice. Following 12 weeks of dietary intervention, HFD feeding increased body weight, liver weight, and white adipose tissue (WAT) (Supplemental Figure 1). 
We observed that HFD+PE mice also had decreased liver and WAT weights than HFD control mice. In addition, PE's effect on the liver function and lipid regulation was assessed. We report that the $45 \% \mathrm{kcal}$ from fat diet increased hepatic total lipid and triglyceride content when compared to a LFD. PE lowered hepatic total lipid and triglyceride content (Supplemental Figure 2). Additionally, PE supplementation decreased hepatic cholesterol and non-esterified fatty acids (Supplemental Figure 2). Taken together, these in vivo findings supported our experimental design, that feeding a diet high in fat would generate a mild metabolic syndrome phenotype in mice. These findings supported further studies into possible diet effects on neuroinflammatory processes.

The addition of PE directly to feed reduced in AD-related genes, key inflammatory mediators, and even some major receptors associated with fatty acid transport/signaling. The inflammatory mediators, IL-1 $\alpha$ and IL-1 $\beta$ were elevated in HFD mice but modulated in PE treated HFD mice. IL-1 has been associated as an initial pro-inflammatory response to infection or damage but more importantly it may be considered the first step in signaling for tissue repair by stimulating helper T-cells (Th17) ${ }^{47,48}$. IL-7 which is heavily involved in B-cell proliferation, T-cell survival, and stimulation of interleukins in monocytes, was augmented in HFD but abrogated in HFD+PE mice ${ }^{49,50}$.

Responsible for protecting epithelial and connective tissue, stimulating neuronal development and serves as a growth factor for erythroid and myeloid progenitor cells, IL-11, was upregulated in HFD mice but significantly attenuated in LFD+PE and HFD+PE mice. The up regulation of this gene as a response to oxidative damage could indicate an attempt at preserving the integrity of the hippocampus and surrounding tissue ${ }^{51}$. 
Interferon $\alpha$ (IFN $\alpha$ ), a pro and anti-inflammatory growth factor, was significantly increased in HFD fed mice but the addition of PE to HFD abrogated gene expression to levels found in LFD controls $(p=0.06)$. TNF $\alpha$, an essential cytokine, growth factor, and intercellular signaling molecule, which is seen largely as a pro-inflammatory molecule was also significantly overexpressed in HFD fed mice but also attenuated to levels similar to LFD control mice. These findings suggest that PE modulates the immune responses associated with prolonged exposure to a HFD ${ }^{14,46}$.

Consistent with findings from other long term diet-induced obesity rodent studies, Tau , GSK3 $\beta$, and APP, were significantly overexpressed in the hippocampi of mice fed a HFD as compared to mice fed a standard LFD $20,52,53$. PE's effect within the hippocampus was seen as largely beneficial, as genes responsible for the production of neurotoxic peptides were significantly reduced. Moreover, our previous studies evaluating these same endpoints but in aged, transgenic $\beta$-amyloid producing mice, demonstrated decreasing trends indicating that the constituents found in PE at the correct dose and exposure time may prevent the translation of proteins that produce neurofibrillary plaques and tangles commonly associated with $A D^{43}$. 
ITGAM, which codes for the CD11b or Macrophage associated receptor -1 (MAC-1) was elevated in HFD mice and facilitates macrophage and microglial phagocytosis of complement coated particles for degradation $^{54,55}$. PE fed HFD mice showed a decrease in ITGAM expression but not significantly. FASL a marker for neuronal cell death was decreased ( although nonstatistically) in PE fed HFD mice by $27.2 \%{ }^{56}$. CD70 appears to have followed a similar trend as FASL, where HFD mice show elevated levels of CD70 expression as compared to LFD controls but the addition of PE to the HFD produced decreasing albeit non-statistically significant trends as compared to HFD alone. Macrophage Inhibitory Factor (MIF) and Colony Stimulating Factor 1 (CSF1) were found to yet again show elevations in HFD mice but with no statistically significant reduction in either gene. Similarly, studies with prolonged PE exposure in transgenic APP/PS1 mouse models, showed attenuation of microgliosis and amyloid plaque deposition ${ }^{57}$

HFD increased the gene expression of Fatty Acid Translocase (FAT) (CD36) and the Leptin Receptor (LEPR) as compared to their LFD fed mice. PE addition to HFD decreased CD36 expression significantly and markedly attenuated LEPR expression as compared to HFD fed mice. glucose and insulin intolerance are two major hallmarks associated with leptin receptor overexpression in mice ${ }^{58}$. Apart from supporting cognitive function, LEPR is implicated in neuronal synaptic plasticity and the food reward response ${ }^{59}$. While serum leptin levels were not measured, literature suggests that low leptin levels in the brain, particularly in the hypothalamus, signal for an increase in food intake ${ }^{60-62}$. Leptin hyposensitivity in specific areas of the brain may cause hyperphagia leading to obesity. Reducing expression or increasing leptin sensitivity may also consequently reduce food intake and drive long term weight loss ${ }^{61,62}$. 
In the hippocampus, neuroinflammatory and neurodegenerative processes are heavily influenced by leptin signaling, and, according to Bonda and colleagues, the result of obesity rather than age ${ }^{63}$. Evidence from other in vivo studies suggests that leptin resistance in Alzheimer's disease is due to hyperphosphorylation of tau by GSK3 $\beta$ and aggregation of neurofibrillary tangles (NFTs) ${ }^{61,62}$. This increase in circulating leptin in the rest of the brain signals for a decrease in food intake and thus promotes weight loss. In hippocampal neurons, aberrant leptin signaling causes cognitive deficits over time ${ }^{62}$.

While this current study shows that APP, MAPT/Tau and GSK3 $\beta$ levels were significantly increased in HFD fed mice, the addition of PE to the HFD significantly reduced the expression of these genes and significantly modulated LEPR signaling as compared to LFD control mice. Additional rodent studies using a number of doses of PE are further needed to confirm the observation that leptin signaling modulation leads to weight loss and an improvement in neuron health in HFD+PE fed mice.

In the present study, PE supplemented LFD mice expression of a number cytokines and receptors such as IL-1 $\beta$, IL-2, IL-3, IL-13, IL-19, NOS2, CD38 and CD70 compared to LFD alone. The expression of these immune factor genes are varied throughout the brain and play distinct roles in specific tissues. For example, IL-1 $\beta$, which is regarded as an initial inflammatory signal, is actually needed to maintain neuronal synaptic plasticity ${ }^{47}$. The increase in the expression of the interleukins $2,3,13$, and 19 which are seen during an influx of lymphocyte infiltration and activation as well as microglial activation may be beneficial and prevent neuronal damage ${ }^{64,65}$. Despite gene expression being elevated, actual protein levels may or may not correlate indicating PE may exert these effects by binding to specific transcription regulatory factors. 
The augmentation of these cytokines, as outlined in Table 1, may induce the differentiation, proliferation, and recruitment of regulatory T-cells (IL-2), basophils, eosinophils (IL-3, 4, 13, 16), CD8 ${ }^{+}$T-cells (IL-9, 16), B-cells (IL-13), NKT cells (IL-15). IL-18 and IL-19 are associated with inducing Th1/Th2 or inducing Th2 cytokine production respectively. Immune cell recruitment is needed in response to cell damage to clear necrotic/apoptotic cells or cellular debris (fibrils, plaques etc.) ${ }^{64,66}$. Recruitment of phagocytic macrophages supported by MIF and CSF1 expression may be indicative of beneficial macrophages or microglia clearing cellular debris. More work in long term diet-induced obesity murine models are needed to confirm this observation that PE induces immune response genes as a means to promote clearance of damaged cells/debris or pathogenic substances (i.e. endotoxin). CD38 or Fatty Acid Translocase in the hippocampus is required in dendritic cell organization and moreover is involved in cyclic ADP ribose and NAD metabolism/catabolism. CD38 knockout models show a protective effect in ischemic brain damage but also leads to an increase in poly ADP ribose (PAR) units and increase in Poly ADP Ribose Glycohydrolase (PARG) ${ }^{67,68}$. An increase in PAR corresponds to an increase in PARP1 activity which may deplete intracellular ATP levels leading to cell death ${ }^{68,69}$. Therefore an increase in CD38 may regulate NAD metabolism and prevent premature neuronal cell death. CD70 is a Cytotoxic T-cell associated antigen that interacts with Helper B-cells through CD27 in order to promote CD8+CTL survival and function ${ }^{70,71}$. This increase in gene expression may promote a diverse population of lymphocytes to clear damaged or dead cell cells within the hippocampus to prevent further inflammation/neurodegeneration. 
Total RNA was isolated from these tissues for the simultaneous quantification and analysis by way of the Luminex xMAP platform. Luminex technology utilizes magnetic beads coated with analyte-specific probes, that, when bound to their target RNA analyte will fluoresce at specific intensities when exposed to red and near infrared laser sources. Beads are assigned a unique bead number and region which allows for each bead to be distinguished. The data acquired from this multiplex is unique in that mean fluorescence intensity or signal is what is amplified and not CDNA as with qRT-PCR ${ }^{72-74}$.

A notable limitation in this study is the inherent biological variability mouse to mouse ${ }^{75}$. Given the implications of gut microbial transformation of these phytochemicals from PE, cohousing effects from four mice per cage, and individual mouse food consumption may have played a role in not only what constituents make it to the brain but at what concentration. Previously, our group has determined that the gut microbial metabolites, urolithins, were the brain penetrable compounds of PE. However, brains of the mice in this study were not perfused $^{76}$.

Given this pilot study utilized a limited number of male mice, future experiments should include an appropriate sample size in order to explore the importance of dose and time on these immunological endpoints and their relationship with a high fat diet with or without PE supplementation. Immunohistochemical staining for microglia and immune cell populations (CD4 ${ }^{+}$vs. $\mathrm{CD}^{+}$, Regulatory T-Cells, Effector T-cells etc.) within the hippocampus, cortex, and cerebellum along with advanced proteomic work of these regions are needed. Proteomics in these regions of the brain will address these findings by quantifying the biologically present proteins from this gene expression panel. 


\section{Conclusions}

Using a diet-induced obesity rodent model, we sought to examine the potential mechanisms associated with PE and obesity associated neuroinflammation. PE was able to modulate several key inflammatory molecules including IL-1 $\alpha, \mathrm{IL}-1 \beta, \mathrm{IL}-7, \mathrm{IL}-11, \mathrm{TNF}, \mathrm{MAPT}$, GSK3 $\beta$, APP and LEPR. PE also augmented several potentially beneficial lymphocyte associated cytokines and antigens including IL-1 $\beta$, IL-2, IL-3, IL-13, IL-19, NOS2, CD38 and CD70. Interestingly several potential pathways could be involved in the beneficial effects derived from PE consumption including the modulating the GS3K $\beta$-TAU-APP pathway, immune cell recruitment/differentiation, and leptin signaling. Further in vitro and in vivo studies are warranted to fully explore PE at various doses and for specific durations in relevant metabolic syndrome or neurodegenerative murine models. 
Funding Acknowledgements

This study was supported through a University of Rhode Island Council for Research Grant.

\section{Author Contributions}

NAD wrote the manuscript, designed, conducted, and analyzed the gene expression

experiments. MP, EM, and HM conducted and oversaw animal care, dosing, sacrifice and feeding. MP and EM designed, conducted and analyzed metabolic endpoint experiments during and concluding the animal study. JA assisted with RNA isolation and RNA quality assessment. AS and NPS conceived the overall study and edited the manuscript.

Conflict of Interest

The authors of this manuscript declare no financial conflict of interest 
Figures
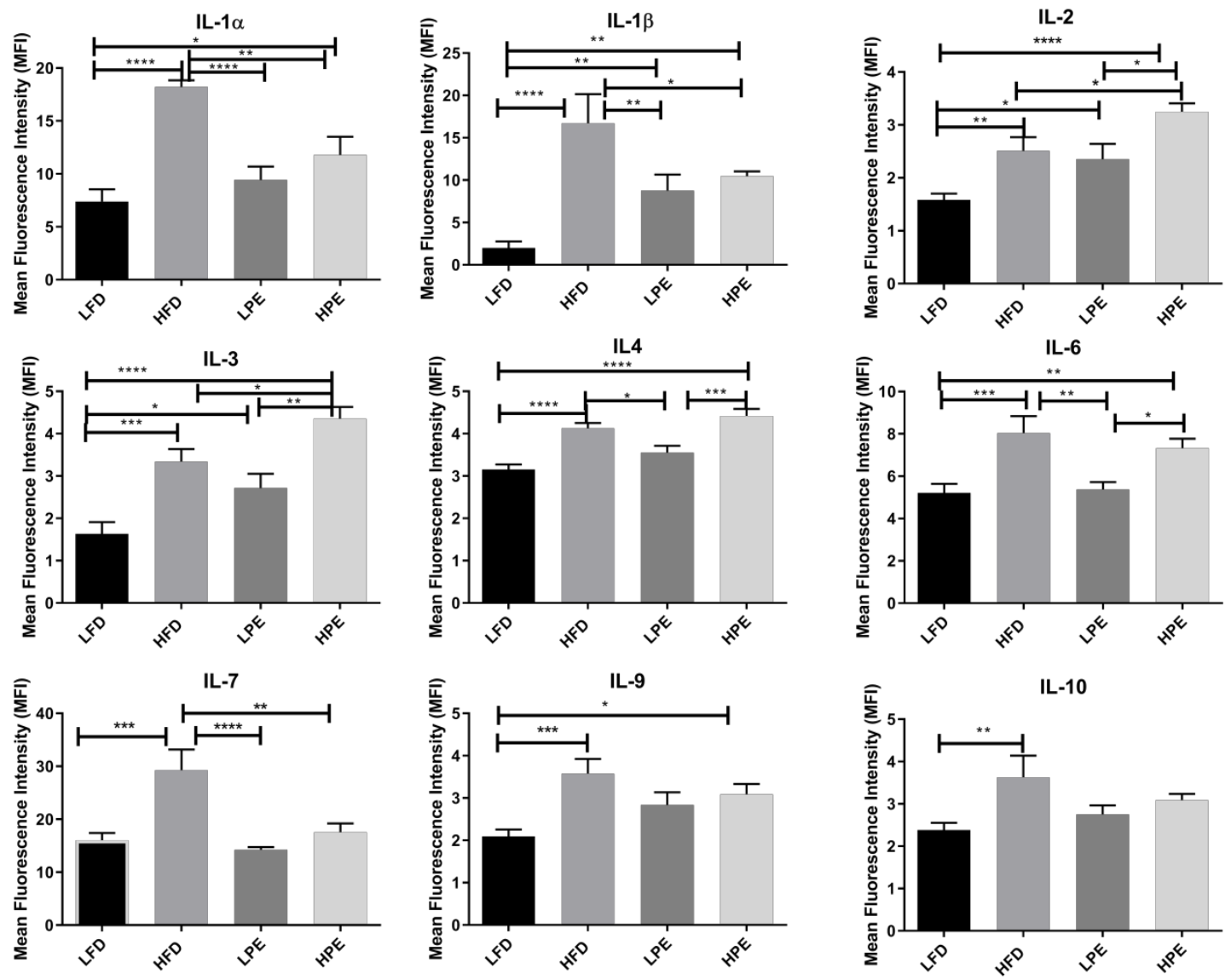

Figure 1.) High Fat Diet and High Fat Diet with PE show a general reduction in proinflammatory Interleukins. HFD mice displayed elevated levels of pro-inflammatory cytokines and chemotractants. PE supplemented HFD mice showed reduced IL-1, IL-6, and IL-7 while also increasing anti-inflammatory cytokines. Statistical significance was calculated using one way ANOVA where $\mathrm{p}<0.05^{*}, \mathrm{p}<0.01 * *, p<0.001^{* * *}, \mathrm{p}<0.0001^{* * * *}$. 

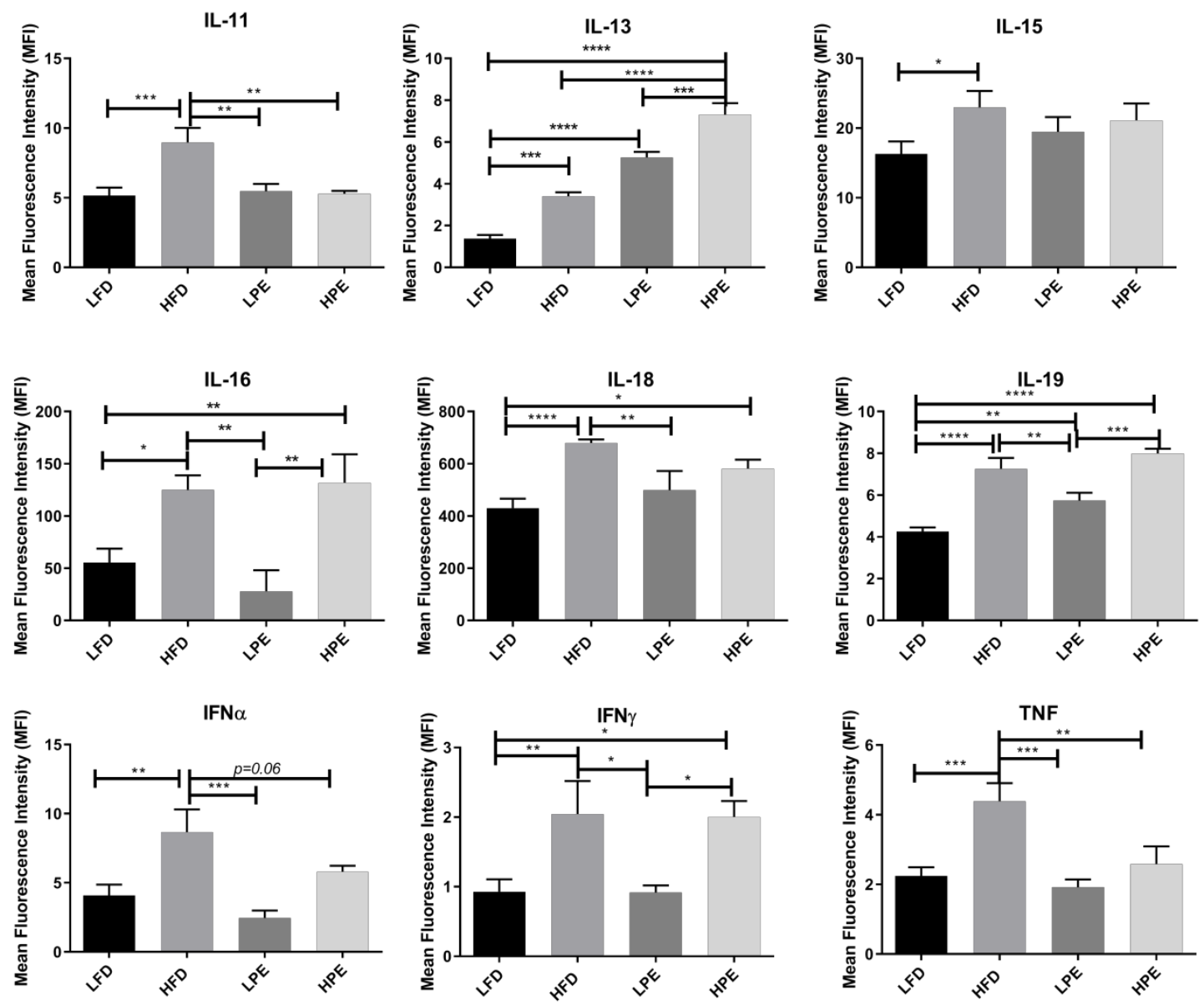

Figure 2.) Pro-inflammatory effects of High Fat Diet consumption are modulated with the inclusion of PE in feed continued. HFD mice displayed elevated levels of pro-inflammatory cytokines and chemotractants. PE supplemented HFD mice showed reduced IL-11, IL-18, IFN $\alpha$, and TNF while also increasing anti-inflammatory cytokines. Statistical significance was calculated using one way ANOVA where $\mathrm{p}<0.05^{*}, \mathrm{p}<0.01^{* *}, \mathrm{p}<0.001^{* * *}, \mathrm{p}<0.0001^{* * * *}$. 

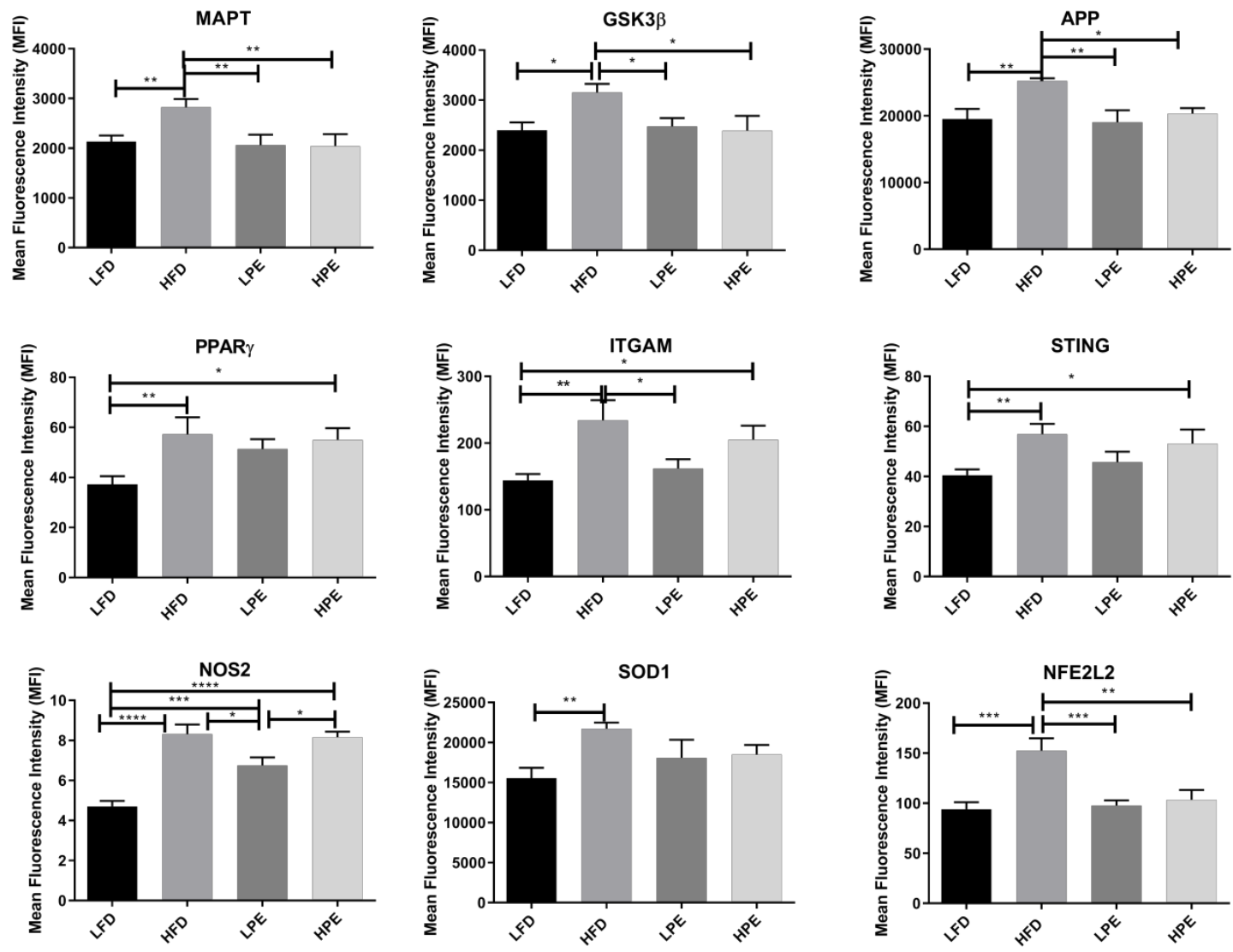

Figure 3.) High Fat Diet Fed Mice show increased levels of AD related genes and anti-oxidant response genes. HFD feeding for 12 weeks increased neurodegenerative associated genes including cytotoxic T-cell chemotractants, and anti-oxidant enzymes. PE supplementation abrogated these markers associated with a HFD intake. Statistical significance was calculated using one way ANOVA where $p<0.05^{*}, p<0.01^{* *}, p<0.001^{* * *}, p<0.0001^{* * * *}$. 

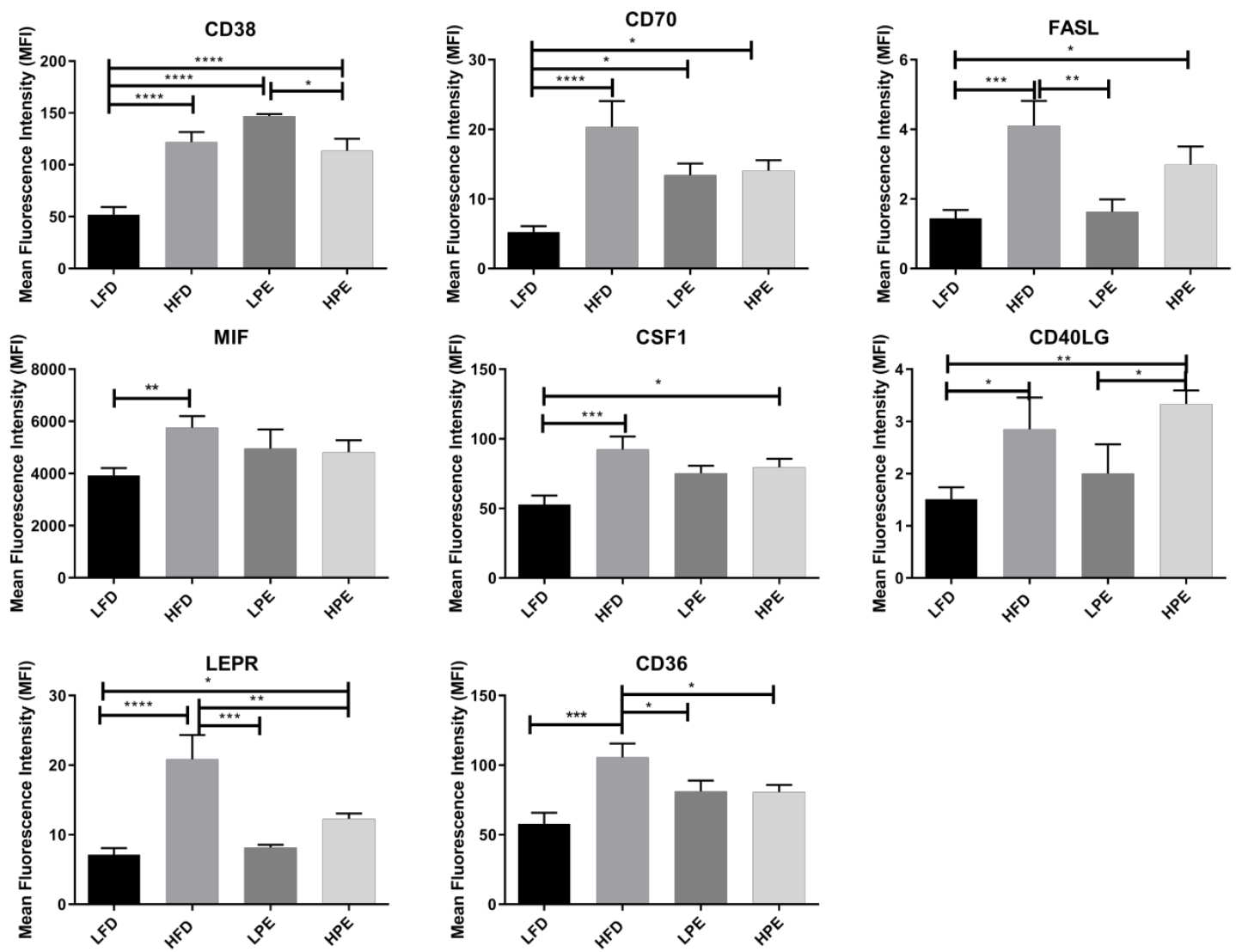

Figure 4.) Macrophage Infiltration, Metabolic Syndrome associated ligand and receptor genes are differentially expressed with and without PE inclusion. Statistical significance was calculated using one way ANOVA where $\mathrm{p}<0.05^{*}, \mathrm{p}<0.01^{* *}, \mathrm{p}<0.001^{* * *}, \mathrm{p}<0.0001^{* * * *}$. 
Table 1.) Multiplex Genes, Function, and Treatment Comparisons

\begin{tabular}{|c|c|c|c|c|c|}
\hline GENE & FUNCTION $^{19,51,54,56,63,67,68,77-83}$ & $\begin{array}{l}\text { HFD vs. } \\
\text { LFD (MEAN } \\
\text { DIFF) }\end{array}$ & $p$ value & $\begin{array}{l}\text { HPE vs. } \\
\text { HFD } \\
\text { (MEAN } \\
\text { DIFF) }\end{array}$ & $p$ value \\
\hline IL-1 $\alpha$ & \multirow{2}{*}{$\begin{array}{l}\text { Induce pro-inflammatory } \\
\text { cytokines and differentiate } \\
\text { Th17 cells }\end{array}$} & 10.81 & $<0.0001$ & -6.425 & 0.0017 \\
\hline IL-1 $\beta$ & & 14.72 & $<0.0001$ & -6.26 & 0.0358 \\
\hline IL-2 & $\begin{array}{l}\text { Stimulate Tregs, T and B } \\
\text { effectors, and NKTs }\end{array}$ & 0.9238 & 0.0021 & 0.7429 & 0.0246 \\
\hline IL-3 & $\begin{array}{c}\text { Activate basophil and } \\
\text { eosinophils, recruit phagocytic } \\
\text { cells }\end{array}$ & 1.708 & 0.0002 & 1.013 & 0.0317 \\
\hline IL-4 & $\begin{array}{l}\text { Induce Th2 cells, induce tissue } \\
\text { adhesion and inflammation }\end{array}$ & 0.975 & $<0.0001$ & 0.2917 & 0.1754 \\
\hline IL-6 & $\begin{array}{l}\text { Infiltration of leukocytes, T-cell } \\
\text { and B-cell differentiation, } \\
\text { survival of cholinergic neurons }\end{array}$ & 2.823 & 0.0007 & -0.7167 & 0.3802 \\
\hline IL-7 & $\begin{array}{l}\text { Proliferation of B-cells (pre and } \\
\text { pro), T-cell survival, induce } \\
\text { Interleukin synthesis in } \\
\text { monocytes }\end{array}$ & 13.22 & 0.0002 & -11.73 & 0.0012 \\
\hline IL-9 & $\begin{array}{l}\text { Growth factor for T and Mast } \\
\text { Cells, Inhibits Th1 cytokines } \\
\text { from CD8 T-cells }\end{array}$ & 1.481 & 0.0003 & -0.4917 & 0.2225 \\
\hline IL-10 & $\begin{array}{c}\text { Immunosuppression via APCs } \\
\text { or T-cells }{ }^{1}\end{array}$ & 1.245 & 0.0056 & -0.5417 & 0.2592 \\
\hline IL-11 & $\begin{array}{l}\text { Growth factor for erythroid, } \\
\text { myeloid and megakaryocyte } \\
\text { progenitors. Protect epithelial } \\
\text { and connective tissue. Inhibits } \\
\text { monocyte and macrophage } \\
\text { activity. Neuronal } \\
\text { development }^{1} \\
\end{array}$ & 3.807 & 0.0004 & -3.687 & 0.0021 \\
\hline IL-13 & $\begin{array}{c}\text { Eosinophil and mast cell } \\
\text { recruitment, activation and } \\
\text { growth. MHC II up regulation } \\
\text { on B cells }\end{array}$ & 2.025 & 0.0002 & 3.9 & $<0.0001$ \\
\hline IL-15 & $\begin{array}{l}\text { T-cell proliferation and } \\
\text { activation (NKT), maintain CD8 } \\
\text { memory, neutrophil and } \\
\text { eosinophil anti-apoptosis }\end{array}$ & 6.667 & 0.026 & -1.837 & 0.5872 \\
\hline IL-16 & $\begin{array}{l}\text { Major chemotractant for } \\
\text { CD4/CD8 T cells, monocytes, } \\
\text { mast cells and eosinophils. }\end{array}$ & 69.56 & 0.0119 & 6.75 & 0.8042 \\
\hline IL-18 & $\begin{array}{c}\text { Induces IFNY with IL-12, } \\
\text { activates Th1 or Th2 }\end{array}$ & 249 & $<0.0001$ & -98.82 & 0.1221 \\
\hline IL-19 & $\begin{array}{l}\text { Induce Th2 cytokines, IL-6, } \\
\text { TNFa and IL-10 in monocytes }\end{array}$ & 3 & $<0.0001$ & 0.733 & 0.17 \\
\hline
\end{tabular}




\begin{tabular}{|c|c|c|c|c|c|}
\hline GENE & FUNCTION $^{19,51,54,56,63,67,68,77-83}$ & $\begin{array}{l}\text { HFD vs. } \\
\text { LFD (MEAN } \\
\text { DIFF) } \\
\end{array}$ & $p$ value & $\begin{array}{l}\text { HPE vs. } \\
\text { HFD } \\
\text { (MEAN } \\
\text { DIFF) } \\
\end{array}$ & $p$ value \\
\hline IFNa & $\begin{array}{l}\text { Viral Response via adaptive } \\
\text { immune signaling, stimulate } \\
\text { DCs antigen presentation, } \\
\text { macrophage AbDC, Promote } \\
\text { naïve T-cell growth and tumor } \\
\text { /virus cell apoptosis }\end{array}$ & 4.572 & 0.0022 & -2.85 & 0.0618 \\
\hline IFNY & $\begin{array}{l}\text { Th1 cell cytotoxic response and } \\
\text { differentiation, upregulates } \\
\text { MHC I and II expression, } \\
\text { inhibits cell growth (epithelial), } \\
\text { pro-apoptosis/cell cycle } \\
\text { modulation. }\end{array}$ & 1.113 & 0.006 & 0.04286 & 0.9193 \\
\hline TNF & $\begin{array}{c}\text { Pro-inflammatory and } \\
\text { immunosuppressive by } \\
\text { initiating or limiting severity } \\
\text { and duration of inflammatory } \\
\text { response. }\end{array}$ & 2.141 & 0.0004 & -1.802 & 0.0041 \\
\hline MAPT & $\begin{array}{l}\text { aka Protein Tau, leads to the } \\
\text { generation of Tau tangles } \\
\text { (immune reactive peptides) }\end{array}$ & 692.7 & 0.0076 & -780.4 & 0.0074 \\
\hline GSK3 $\beta$ & $\begin{array}{c}\text { Neuronal development via } \\
\text { neuronal progenitor } \\
\text { differentiation. }^{3}\end{array}$ & 751.8 & 0.0106 & -761.5 & 0.0161 \\
\hline APP & $\begin{array}{l}\text { Amyloid Precursor protein, } \\
\text { normally involved in neuronal } \\
\text { plasticity and neuronal } \\
\text { development. Over expression } \\
\text { leads to plaque formation and } \\
\text { microglial activation }\end{array}$ & 5721 & 0.0057 & 4883 & 0.0369 \\
\hline PPARY & $\begin{array}{l}\text { Neuronal depression of } \\
\text { inflammation associated with } \\
\text { chronic or acute insult. Glucose } \\
\text { absorption, lipid balance and } \\
\text { cell growth/differentiation }\end{array}$ & 20 & 0.0073 & -2.249 & 0.7622 \\
\hline ITGAM & $\begin{array}{l}\text { Leukocyte adhesion, aka } \\
\text { Macrophage Receptor } 1 \text {, } \\
\text { upregulated by TNF, mediate } \\
\text { complement coated } \\
\text { macrophage uptake of } \\
\text { particles for degradation. }\end{array}$ & 90.02 & 0.0026 & -29.37 & 0.3086 \\
\hline STING & $\begin{array}{c}\text { Stimulates Interferon Genes in } \\
\text { response to viral or internal } \\
\text { pathogens (microbial DNA). } \\
\text { Localizes with autophagy } \\
\text { related proteins }\end{array}$ & 16.42 & 0.0032 & -3.786 & 0.518 \\
\hline NOS2 & $\begin{array}{l}\text { Induced by IFN } \gamma \text {, TNF and IL- } \\
1 \beta . \text { Macrophage associated NO } \\
\text { production, stimulates IL-6 and }\end{array}$ & 3.604 & $<0.0001$ & -0.16 & 0.7743 \\
\hline
\end{tabular}




\begin{tabular}{|c|c|c|c|c|c|}
\hline GENE & FUNCTION $^{19,51,54,56,63,67,68,77-83}$ & $\begin{array}{l}\text { HFD vs. } \\
\text { LFD (MEAN } \\
\text { DIFF) } \\
\end{array}$ & $p$ value & $\begin{array}{l}\text { HPE vs. } \\
\text { HFD } \\
\text { (MEAN } \\
\text { DIFF) }\end{array}$ & p value \\
\hline & $\begin{array}{l}\text { IL-8, stimulate epithelial cell } \\
\text { growth }\end{array}$ & & & & \\
\hline SOD1 & $\begin{array}{c}\text { Anti-oxidant response by } \\
\text { binding to superoxide radial } \\
\text { molecules }\end{array}$ & 6170 & 0.0038 & -3242 & 0.1289 \\
\hline NFEL2 & $\begin{array}{l}\text { aka NRF2, interacts with anti- } \\
\text { oxidant response element, } \\
\text { stimulating Phase II anti- } \\
\text { oxidant response enzymes } \\
\text { (HO-1 and NQO-1) }\end{array}$ & 58.44 & 0.0001 & -49.03 & 0.0024 \\
\hline CD38 & $\begin{array}{c}\text { TNFa inducible, Dendritic } \\
\text { organization in hippocampal } \\
\text { excitatory neurons, humoral } \\
\text { immune response }\end{array}$ & 70.13 & $<0.0001$ & -8.63 & 0.5228 \\
\hline CD70 & $\begin{array}{l}\text { TNF ligand, activates T and B } \\
\text { cells, improves cytotoxic } \\
\text { function of NK cells, } \\
\text { proliferation of CD8 T cells }\end{array}$ & 15.11 & $<0.0001$ & -6.3 & 0.0764 \\
\hline FASL & $\begin{array}{l}\text { TNF ligand, forms Death } \\
\text { Domain Complex leading to } \\
\text { caspase } 8 \text { mediated apoptosis }\end{array}$ & 2.66 & 0.0002 & -1.117 & 0.1106 \\
\hline MIF & $\begin{array}{c}\text { Macrophage Migration } \\
\text { Inhibitor Factor, response to } \\
\text { bacterial infection (LPS or } \\
\text { DNA), stimulates cytokine } \\
\text { release in macrophages }\end{array}$ & 1824 & 0.008 & -936.9 & 0.1819 \\
\hline CSF1 & $\begin{array}{c}\text { Colony Stimulating Factor -1 } \\
\text { induces macrophage } \\
\text { infiltration, induces pro- } \\
\text { inflammatory cytokine } \\
\text { secretion }\end{array}$ & 39.53 & 0.0008 & -12.74 & 0.2549 \\
\hline CD40LG & $\begin{array}{l}\text { TNF ligand, stimulate T-cell } \\
\text { growth and cytokine synthesis } \\
\text { (IL-4 and IL-10) }\end{array}$ & 1.339 & 0.0217 & 0.4833 & 0.4241 \\
\hline LEPR & $\begin{array}{l}\text { Neuronal stimulation and } \\
\text { plasticity, food reward (anti- } \\
\text { inflammatory).Pro- } \\
\text { inflammatory and increased } \\
\text { with HFD or metabolic } \\
\text { syndrome }\end{array}$ & 13.71 & $<0.0001$ & -8.55 & 0.0039 \\
\hline CD36 & $\begin{array}{l}\text { Aka FAT, transport fatty acids } \\
\text { into and out of wide range of } \\
\text { cells. Inhibited by LPS and HDL, } \\
\text { induced by cholesterol, CSF, IL- } \\
4 \text {, insulin and glucose. }\end{array}$ & 47.9 & 0.0003 & -25.13 & 0.0399 \\
\hline
\end{tabular}


A

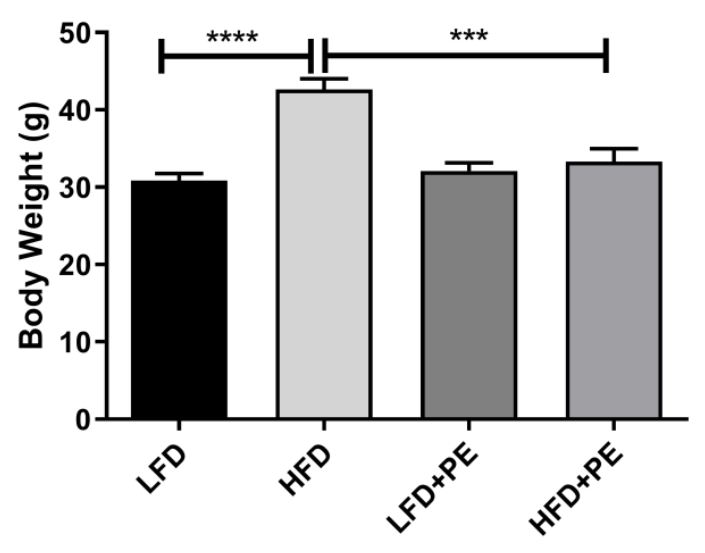

B

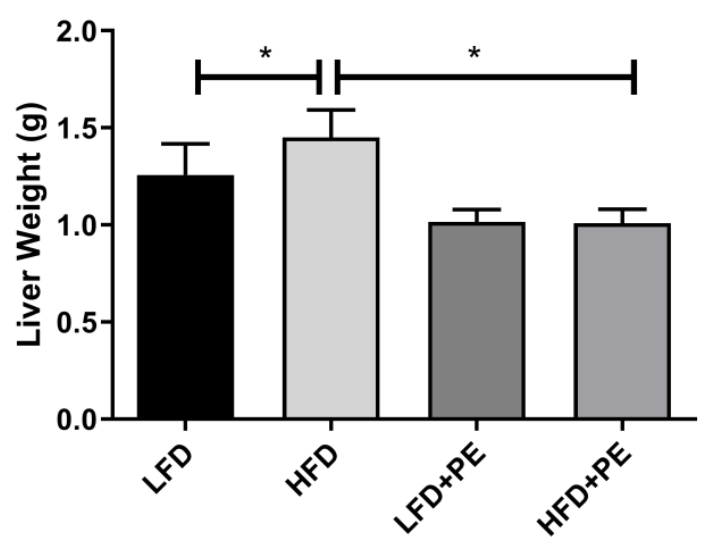

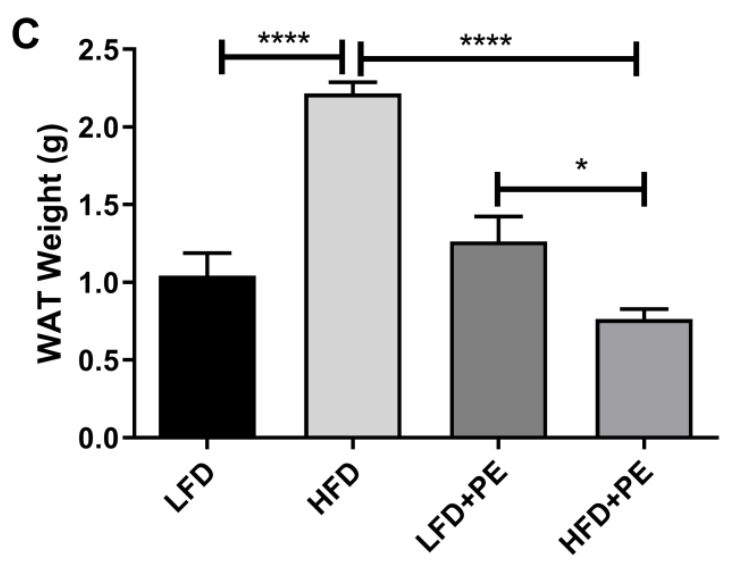

Supplemental Figure 1.) High Fat and Low Fat Diet Effects on Body, White Adipose, and Liver Weight with and without PE supplementation. Statistical significance was calculated using one way ANOVA with Fisher's LSD test where $p<0.05^{*}, p<0.01^{* *}, p<0.001^{* * *}, p<0.0001^{* * * *}$. 


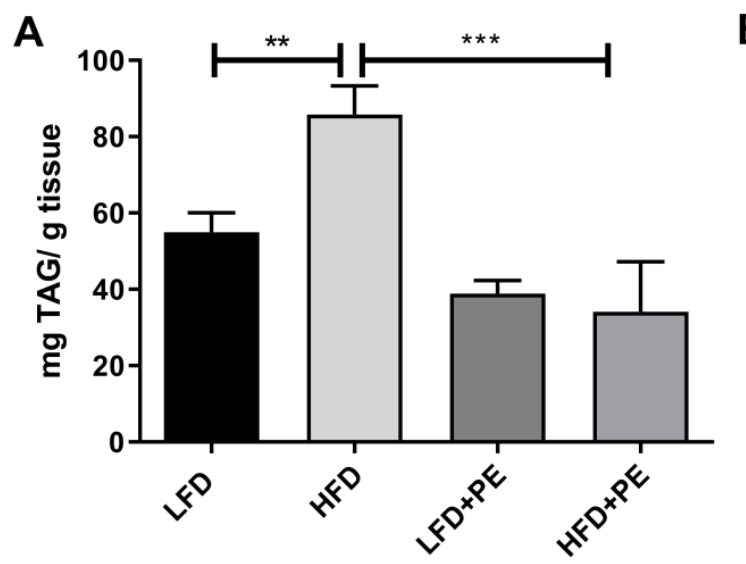

B

C

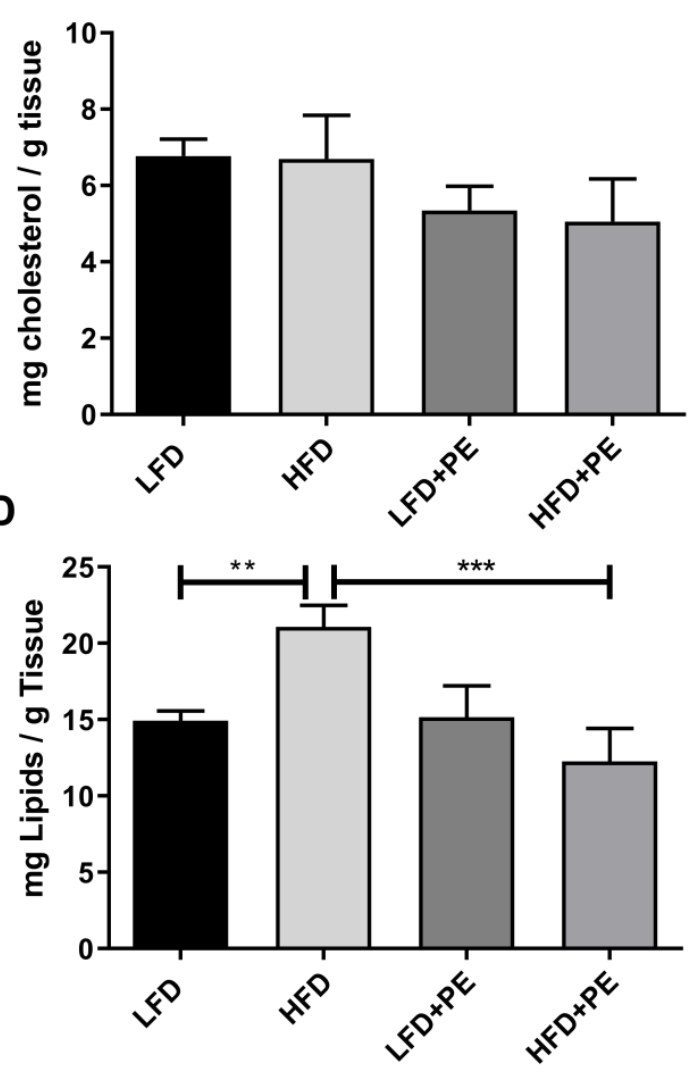

Supplemental Figure 2.) Hepatic Triacylglycerides, Total Lipids, and Non-esterified Free Fatty Acids are abrogated with PE supplementation in both High Fat and Low Fat Diet fed mice.

Statistical significance was calculated using one way ANOVA with fisher's LSD test where $p<0.05$ $*, p<0.01 * *, p<0.001 * * *, p<0.0001 * * * *$. 


\section{References and Works Cited}

1. Wolowczuk, I. Obesity - an inflammatory state. Acta Vet. Scand. 57, K5 (2015).

2. Glaros, T. G. et al. Causes and consequences of low grade endotoxemia and inflammatory diseases. Front. Biosci. Sch. Ed. 5, 754-765 (2013).

3. Frick, J.-S. \& Autenrieth, I. B. The gut microflora and its variety of roles in health and disease. Curr. Top. Microbiol. Immunol. 358, 273-289 (2013).

4. Boutagy, N. E., McMillan, R. P., Frisard, M. I. \& Hulver, M. W. Metabolic endotoxemia with obesity: Is it real and is it relevant? Biochimie 124, 11-20 (2016).

5. Fändriks, L. Roles of the gut in the metabolic syndrome: an overview. J. Intern. Med. 281, 319-336 (2017).

6. Faraj, T. A., McLaughlin, C. L. \& Erridge, C. Host defenses against metabolic endotoxaemia and their impact on lipopolysaccharide detection. Int. Rev. Immunol. 36, 125-144 (2017).

7. Moreira, A. P. B., Texeira, T. F. S., Ferreira, A. B., Peluzio, M. do C. G. \& Alfenas, R. de C. G. Influence of a high-fat diet on gut microbiota, intestinal permeability and metabolic endotoxaemia. Br. J. Nutr. 108, 801-809 (2012).

8. Guillemot-Legris, O. \& Muccioli, G. G. Obesity-Induced Neuroinflammation: Beyond the Hypothalamus. Trends Neurosci. 40, 237-253 (2017).

9. Glass, C. K., Saijo, K., Winner, B., Marchetto, M. C. \& Gage, F. H. Mechanisms Underlying Inflammation in Neurodegeneration. Cell 140, 918-934 (2010).

10. Zhang, R. et al. Circulating endotoxin and systemic immune activation in sporadic Amyotrophic Lateral Sclerosis (sALS). J. Neuroimmunol. 206, 121-124 (2009).

11. Leicht, H. et al. Predictors of costs in dementia in a longitudinal perspective. PloS One 8, e70018 (2013).

12. Madabhushi, R., Pan, L. \& Tsai, L.-H. DNA damage and its links to neurodegeneration. Neuron 83, 266-282 (2014). 
13. Deczkowska, A. et al. Disease-Associated Microglia: A Universal Immune Sensor of Neurodegeneration. Cell 173, 1073-1081 (2018).

14. Rogers, J. et al. Inflammation and Alzheimer's disease pathogenesis. Neurobiol. Aging 17, 681-686 (1996).

15. McGeer, P. L., Itagaki, S., Boyes, B. E. \& McGeer, E. G. Reactive microglia are positive for HLA-DR in the substantia nigra of Parkinson's and Alzheimer's disease brains. Neurology 38, 1285-1291 (1988).

16. Mueller, S. G. et al. Hippocampal Atrophy Patterns in Mild Cognitive Impairment and Alzheimer's Disease. Hum. Brain Mapp. 31, 1339-1347 (2010).

17. Schröder, J. \& Pantel, J. Neuroimaging of hippocampal atrophy in early recognition of Alzheimer's disease--a critical appraisal after two decades of research. Psychiatry Res. Neuroimaging 247, 71-78 (2016).

18. Avila, J., Gómez de Barreda, E., Engel, T., Lucas, J. J. \& Hernández, F. Tau phosphorylation in hippocampus results in toxic gain-of-function. Biochem. Soc. Trans. 38, 977-980 (2010).

19. Sebastián-Serrano, Á., de Diego-García, L. \& Díaz-Hernández, M. The Neurotoxic Role of Extracellular Tau Protein. Int. J. Mol. Sci. 19, (2018).

20. Del Olmo, N. \& Ruiz-Gayo, M. Influence of High-Fat Diets Consumed During the Juvenile Period on Hippocampal Morphology and Function. Front. Cell. Neurosci. 12, (2018).

21. Polyzos, S. A., Kountouras, J. \& Mantzoros, C. S. Obesity and nonalcoholic fatty liver disease: From pathophysiology to therapeutics. Metabolism (2018).

doi:10.1016/j.metabol.2018.11.014

22. Takahashi, Y., Sugimoto, K., Inui, H. \& Fukusato, T. Current pharmacological therapies for nonalcoholic fatty liver disease/nonalcoholic steatohepatitis. World J. Gastroenterol. WJG 21, 3777-3785 (2015).

23. Eshraghian, A. Current and emerging pharmacological therapy for non-alcoholic fatty liver disease. World J. Gastroenterol. 23, 7495-7504 (2017). 
24. Moelands, S. V., Lucassen, P. L., Akkermans, R. P., De Grauw, W. J. \& Van de Laar, F. A. Alpha-glucosidase inhibitors for prevention or delay of type 2 diabetes mellitus and its associated complications in people at increased risk of developing type 2 diabetes mellitus. Cochrane Database Syst. Rev. 12, CD005061 (2018).

25. Honda, Y. et al. Pemafibrate, a novel selective peroxisome proliferator-activated receptor alpha modulator, improves the pathogenesis in a rodent model of nonalcoholic steatohepatitis. Sci. Rep. 7, 42477 (2017).

26. Deardorff, W. J. \& Grossberg, G. T. Targeting neuroinflammation in Alzheimer's disease: evidence for NSAIDs and novel therapeutics. Expert Rev. Neurother. 17, 17-32 (2017).

27. Dou, K.-X. et al. Comparative safety and effectiveness of cholinesterase inhibitors and memantine for Alzheimer's disease: a network meta-analysis of 41 randomized controlled trials. Alzheimers Res. Ther. 10, 126 (2018).

28. Salomone, F., Godos, J. \& Zelber-Sagi, S. Natural antioxidants for non-alcoholic fatty liver disease: molecular targets and clinical perspectives. Liver Int. Off. J. Int. Assoc. Study Liver 36, 5-20 (2016).

29. Cicero, A. F. G., Colletti, A. \& Bellentani, S. Nutraceutical Approach to Non-Alcoholic Fatty Liver Disease (NAFLD): The Available Clinical Evidence. Nutrients 10, (2018).

30. Salgueiro, A. C. F. et al. Predictive antidiabetic activities of plants used by persons with Diabetes mellitus. Complement. Ther. Med. 41, 1-9 (2018).

31. Naseri, R. et al. Anthocyanins in the Management of Metabolic Syndrome: A Pharmacological and Biopharmaceutical Review. Front. Pharmacol. 9, 1310 (2018).

32. Amiot, M. J., Riva, C. \& Vinet, A. Effects of dietary polyphenols on metabolic syndrome features in humans: a systematic review. Obes. Rev. Off. J. Int. Assoc. Study Obes. 17, 573586 (2016).

33. Amor, S., Châlons, P., Aires, V. \& Delmas, D. Polyphenol Extracts from Red Wine and Grapevine: Potential Effects on Cancers. Dis. Basel Switz. 6, (2018).

34. Chaplin, A., Carpéné, C. \& Mercader, J. Resveratrol, Metabolic Syndrome, and Gut Microbiota. Nutrients 10, (2018). 
35. DiSilvestro, R. A., DiSilvestro, D. J. \& DiSilvestro, D. J. Pomegranate extract mouth rinsing effects on saliva measures relevant to gingivitis risk. Phytother. Res. 23, 1123-1127 (2009).

36. Pacheco-Palencia, L. A., Noratto, G., Hingorani, L., Talcott, S. T. \& Mertens-Talcott, S. U. Protective Effects of Standardized Pomegranate (Punica granatum L.) Polyphenolic Extract in Ultraviolet-Irradiated Human Skin Fibroblasts. J. Agric. Food Chem. 56, 8434-8441 (2008).

37. Mertens-Talcott, S. U., Jilma-Stohlawetz, P., Rios, J., Hingorani, L. \& Derendorf, H. Absorption, Metabolism, and Antioxidant Effects of Pomegranate (Punica granatum L.) Polyphenols after Ingestion of a Standardized Extract in Healthy Human Volunteers. J. Agric. Food Chem. 54, 8956-8961 (2006).

38. Sohrab, G. et al. Pomegranate (Punica granatum) juice decreases lipid peroxidation, but has no effect on plasma advanced glycated end-products in adults with type 2 diabetes: a randomized double-blind clinical trial. Food Nutr. Res. 59, (2015).

39. Larrosa, M. et al. Anti-inflammatory properties of a Pomegranate extract and its metabolite urolithin-A in a colitis rat model and the effect of colon inflammation on phenolic metabolism. J. Nutr. Biochem. 21, 717-725 (2010).

40. Espín, J. C., Larrosa, M., García-Conesa, M. T. \& Tomás-Barberán, F. Biological significance of urolithins, the gut microbial ellagic Acid-derived metabolites: the evidence so far. Evid.Based Complement. Altern. Med. ECAM 2013, 270418 (2013).

41. DaSilva, N. A. et al. Pomegranate ellagitannin-gut microbial-derived metabolites, urolithins, inhibit neuroinflammation in vitro. Nutr. Neurosci. 1-11 (2017).

doi:10.1080/1028415X.2017.1360558

42. Xu, J. et al. Urolithins Attenuate LPS-Induced Neuroinflammation in BV2Microglia via MAPK, Akt, and NF-kB Signaling Pathways. J. Agric. Food Chem. 66, 571-580 (2018).

43. Ahmed, A. H. et al. Pomegranate extract modulates processing of amyloid- $\beta$ precursor protein in an aged Alzheimer's disease animal model. Curr. Alzheimer Res. 11, 834-843 (2014).

44. More, V. R. et al. Keap1 knockdown increases markers of metabolic syndrome after longterm high fat diet feeding. Free Radic. Biol. Med. 61, 85-94 (2013). 
45. Zlokovic, B. V. The blood-brain barrier in health and chronic neurodegenerative disorders. Neuron 57, 178-201 (2008).

46. Ransohoff, R. M. How neuroinflammation contributes to neurodegeneration. Science 353, 777-783 (2016).

47. Sobesky, J. L. et al. High-fat diet consumption disrupts memory and primes elevations in hippocampal IL-1 $\beta$, an effect that can be prevented with dietary reversal or IL-1 receptor antagonism. Brain. Behav. Immun. 42, 22-32 (2014).

48. Chhor, V. et al. Characterization of phenotype markers and neuronotoxic potential of polarised primary microglia in vitro. Brain. Behav. Immun. 32, 70-85 (2013).

49. Lucas, S. et al. Interleukin-7 Regulates Adipose Tissue Mass and Insulin Sensitivity in HighFat Diet-Fed Mice through Lymphocyte-Dependent and Independent Mechanisms. PLOS ONE 7, e40351 (2012).

50. Araujo, D. M. \& Cotman, C. W. Trophic effects of interleukin-4, -7 and -8 on hippocampal neuronal cultures: potential involvement of glial-derived factors. Brain Res. 600, 49-55 (1993).

51. Paul, S. R. \& Schendel, P. The cloning and biological characterization of recombinant human interleukin 11. Int. J. Cell Cloning 10, 135-143 (1992).

52. Cai, D. Neuroinflammation and neurodegeneration in overnutrition-induced diseases. Trends Endocrinol. Metab. TEM 24, 40-47 (2013).

53. Nuzzo, D. et al. Insulin Resistance as Common Molecular Denominator Linking Obesity to Alzheimer's Disease. Curr. Alzheimer Res. 12, 723-735 (2015).

54. Pei, Z. et al. MAC1 mediates LPS-induced production of superoxide by microglia: the role of pattern recognition receptors in dopaminergic neurotoxicity. Glia 55, 1362-1373 (2007).

55. Bodea, L.-G. et al. Neurodegeneration by Activation of the Microglial ComplementPhagosome Pathway. J. Neurosci. 34, 8546-8556 (2014). 
56. Grosjean, M. B. et al. Immunohistochemical characterization of Fas (CD95) and Fas Ligand (FasL/CD95L) expression in the injured brain: relationship with neuronal cell death and inflammatory mediators. Histol. Histopathol. 22, 235-250 (2007).

57. Rojanathammanee, L., Puig, K. L. \& Combs, C. K. Pomegranate polyphenols and extract inhibit nuclear factor of activated T-cell activity and microglial activation in vitro and in a transgenic mouse model of Alzheimer disease. J. Nutr. 143, 597-605 (2013).

58. Amitani, M., Asakawa, A., Amitani, H. \& Inui, A. The role of leptin in the control of insulinglucose axis. Front. Neurosci. 7, (2013).

59. Morris, D. L. \& Rui, L. Recent advances in understanding leptin signaling and leptin resistance. Am. J. Physiol. Endocrinol. Metab. 297, E1247-1259 (2009).

60. Scott, M. M. et al. Leptin Targets in the Mouse Brain. J. Comp. Neurol. 514, 518-532 (2009).

61. Bjørbæk, C. Central Leptin Receptor Action and Resistance in Obesity. J. Investig. Med. Off. Publ. Am. Fed. Clin. Res. 57, 789-794 (2009).

62. McGregor, G. \& Harvey, J. Food for thought: Leptin regulation of hippocampal function and its role in Alzheimer's disease. Neuropharmacology 136, 298-306 (2018).

63. Bonda, D. J. et al. Dysregulation of leptin signaling in Alzheimer disease: evidence for neuronal leptin resistance. J. Neurochem. 128, 162-172 (2014).

64. González, H. \& Pacheco, R. T-cell-mediated regulation of neuroinflammation involved in neurodegenerative diseases. J. Neuroinflammation 11, 201 (2014).

65. Brochard, V. et al. Infiltration of CD4+ lymphocytes into the brain contributes to neurodegeneration in a mouse model of Parkinson disease. J. Clin. Invest. 119, 182-192 (2009).

66. Appel, S. H. CD4+ T cells mediate cytotoxicity in neurodegenerative diseases. J. Clin. Invest. 119, 13-15 (2009).

67. Nelissen, T. P. et al. CD38 is Required for Dendritic Organization in Visual Cortex and Hippocampus. Neuroscience 372, 114-125 (2018). 
68. Long, A. et al. CD38 Knockout Mice Show Significant Protection Against Ischemic Brain Damage Despite High Level Poly-ADP-Ribosylation. Neurochem. Res. 42, 283-293 (2017).

69. Strosznajder, R. P., Czubowicz, K., Jesko, H. \& Strosznajder, J. B. Poly(ADP-ribose) metabolism in brain and its role in ischemia pathology. Mol. Neurobiol. 41, 187-196 (2010).

70. Deola, S. et al. Helper B cells promote cytotoxic T cell survival and proliferation independently of antigen presentation through CD27/CD70 interactions. J. Immunol. Baltim. Md 1950 180, 1362-1372 (2008).

71. Rowley, T. F. \& Al-Shamkhani, A. Stimulation by soluble CD70 promotes strong primary and secondary CD8+ cytotoxic T cell responses in vivo. J. Immunol. Baltim. Md 1950 172, 60396046 (2004).

72. Coles, A. H. et al. A High-Throughput Method for Direct Detection of Therapeutic Oligonucleotide-Induced Gene Silencing In Vivo. Nucleic Acid Ther. 26, 86-92 (2016).

73. Chen, L. et al. TLR4 inhibitor attenuates amyloid- $\beta$-induced angiogenic and inflammatory factors in ARPE-19 cells: Implications for age-related macular degeneration. Mol. Med. Rep. 13, 3249-3256 (2016).

74. Tai, L. M. et al. APOE-modulated A $\beta$-induced neuroinflammation in Alzheimer's disease: current landscape, novel data, and future perspective. J. Neurochem. 133, 465-488 (2015).

75. Miyoshi, J. et al. Minimizing confounders and increasing data quality in murine models for studies of the gut microbiome. PeerJ 6, e5166 (2018).

76. Yuan, T. et al. Pomegranate's Neuroprotective Effects against Alzheimer's disease Are Mediated by Urolithins, Its Ellagitannin-Gut Microbial Derived Metabolites. ACS Chem. Neurosci. 7, 26-33 (2016).

77. Akdis, M. et al. Interleukins (from IL-1 to IL-38), interferons, transforming growth factor $\beta$, and TNF- $\alpha$ : Receptors, functions, and roles in diseases. J. Allergy Clin. Immunol. 138, 9841010 (2016).

78. Dmitrieva, O. S., Shilovskiy, I. P., Khaitov, M. R. \& Grivennikov, S. I. Interleukins 1 and 6 as Main Mediators of Inflammation and Cancer. Biochem. Biokhimiia 81, 80-90 (2016). 
79. Liu, E. et al. GSK-3ß deletion in dentate gyrus excitatory neuron impairs synaptic plasticity and memory. Sci. Rep. 7, 5781 (2017).

80. Baranello, R. J. et al. Amyloid-beta protein clearance and degradation ( $A B C D)$ pathways and their role in Alzheimer's disease. Curr. Alzheimer Res. 12, 32-46 (2015).

81. Varga, T., Czimmerer, Z. \& Nagy, L. PPARs are a unique set of fatty acid regulated transcription factors controlling both lipid metabolism and inflammation. Biochim. Biophys. Acta 1812, 1007-1022 (2011).

82. Simons, K. H. et al. The role of CD27-CD70-mediated T cell co-stimulation in vasculogenesis, arteriogenesis and angiogenesis. Int. J. Cardiol. 260, 184-190 (2018).

83. Morrison, C. D. et al. High Fat Diet Increases Hippocampal Oxidative Stress and Cognitive Impairment in Aged Mice: Implications for decreased Nrf2 signaling. J. Neurochem. 114, 1581-1589 (2010). 


\section{MANUSCRIPT 2}

\section{In preparation for submission to Nutritional Neuroscience}

Effects of a food grade, phenol-enriched maple syrup extract on hippocampus tissue from diet-induced obese C57BL/6 mice.

Nicholas A. DaSilva ${ }^{1}$, Marisa Pfohl ${ }^{1}$, Emily Marques ${ }^{1}$, Hang Ma ${ }^{1}$, Angela L. Slitt ${ }^{1,2}$, Navindra P. Seeram $^{1,2}$

Affiliations:

${ }^{1}$ Department of Biomedical and Pharmaceutical Sciences, University of Rhode Island, Kingston, RI USA

${ }^{2}$ George and Anne Ryan Institute for Neuroscience, University of Rhode Island, Kingston, RI USA Corresponding Authors:

Navindra Seeram, Ph.D.; University of Rhode Island, 495B, Avedisian Hall, 7 Greenhouse Rd. Kingston, RI, 02881, USA, Phone: 401-874-9367, Email: nseeram@uri.edu Angela L. Slitt, Ph.D.; University of Rhode Island, 395D, Avedisian Hall, 7 Greenhouse Rd. Kingston, RI 02881, USA, Phone: 401-874-5020, Email: aslitt@uri.edu 


\section{Abstract}

Background: Diet-induced obesity is associated with increased circulating levels of endotoxin and chronic low grade inflammation. This inflammatory source is linked to the activation and recruitment of microglia and is part of the causal link to the pathogenesis of a number of neurodegenerative diseases such as Alzheimer's, Parkinson's and Amyotrophic Lateral Sclerosis. Maple Syrup Extract (MSX), could be a potential source of beneficial phytonutrients capable of mitigating these inflammatory processes from occurring.

Methods: A pilot scale exploratory study with Male C57BL/6 mice fed either a standard grain free rodent diet ( $10 \% \mathrm{kcal}$ from fat) or a high fat diet ( $45 \% \mathrm{kcal}$ from fat) with or without MSX at a dose of $0.5 \% \mathrm{w} / \mathrm{w}$ incorporated directly into feed for 12 weeks to yield an aged diet-induced obesity phenotype. Mice were sacrificed following 12 weeks and whole hippocampi were excised for Luminex xMAP based, QuantiGene multiplex gene expression analysis of inflammatory and neurodegenerative disease associated genes.

Results: Mice fed a HFD for 12 weeks showed elevated expression of Alzheimer's Disease related genes aswell as both pro and anti-inflammatory cytokine/growth factors in hippocampal tissues. MSX supplementation in HFD feed allowed for a significant reduction in the gene expression of IL-7, IL-11, IL-19, TNF $\alpha$, IFN $\alpha$, CD40L, CD70, NFEL2, SOD1, ITGAM, and even LEPR. Conclusions: The number of constituents that make up MSX could play multiple roles in organ systems. In the hippocampus, MSX may act on not only pro-inflammatory gene expression but TNF mediated death receptor pathways in addition to modulating leptin receptor signaling. 


\section{Background}

Obesity and its associated diseases (Type II Diabetes Mellitus (T2DM), Cardiovascular Disease, NAFLD, NASH and even cancer) represent a global healthcare and economic burden ${ }^{1}$. Poor diet has been linked to the generation of low grade inflammation systemically ${ }^{2}$. The gut and its numerous microbial inhabitants have been found to be a major source of proinflammatory molecules such as lipopolysaccharide (LPS) ${ }^{3}$. These pro-inflammatory molecules trigger the activation of macrophages which triggers the recruitment and activation of additional lymphocytes and leukocytes ${ }^{4,5}$. The prototypical endotoxin, LPS, which has been found in the plasma of obese individuals, has also been found in those diagnosed with neurodegenerative diseases such as Alzheimer's disease (AD), Parkinson's disease (PD) and even Amyotrophic Lateral Sclerosis $(\mathrm{ALS})^{6-8}$. These neurodegenerative diseases are associated with their own set of unique and challenging healthcare needs while also adding to the global economic burden associated with an ageing population ${ }^{9}$.

Neurodegeneration, or the progressive loss in neuron function and viability, is associated with ageing and neuroinflammation ${ }^{10}$. Neuroinflammation is largely governed by the resident immune cells of the brain, microglia ${ }^{11}$. Over time, the brain is insulted with endotoxins and environmental toxicants (e.g. Lead, DDT, Mercury etc) ${ }^{12,13}$. The Hippocampus, which is the center of neurogenesis in the developing brain, is responsible for maintaining basic cognitive function and memory, and is one of the first regions to be affected by such insult. The hippocampus is also a major site of neurotoxic neurofibrillary plaques and tangles accumulation ${ }^{13-15}$. We can accelerate the ageing process in mice through a diet-induced obesity model by feeding mice a diet rich in saturated fats. This effectively models the typical "Western" diet of obese individuals in North America and Europe ${ }^{16}$. 
Therapeutic intervention with, insulin sensitizers such as Metformin and

Thiazolidinediones (e.g. rosiglitazone), lipid and cholesterol reducers (atorvastatin, simvastatin), alpha-glucosidase inhibitors, Non-steroidal Anti Inflammatory Drugs (NSAIDs) and cholinesterase Inhibitors induce a number of harmful side effects ${ }^{17-23}$. Complementary and natural products in the form of functional foods or herbal medicines have also been considered for patients with metabolic syndrome (MetS) on the basis of safety and cost. Despite the number of clinical studies involving natural products for the management of MetS have been published, our understanding of the actual mechanisms of these products in the brain are still being elucidated ${ }^{24,25}$. Of the diverse classes of natural products studied, polyphenols are by far the most significantly studied ${ }^{26,27}$.

Polyphenols from a novel, food grade extract derived from a polyphenol rich grade of maple syrup termed MSX. MSX has been previously found to have over 60 phytochemicals representing a diverse range of polyphenol classes such as Lignans, Coumarins, Phenolic Acids and Stilbenes. MSX has been tested in rats at doses up to $1000 \mathrm{mg} / \mathrm{kg}$ per day with no observable signs of toxicity ${ }^{28}$. MSX has also been studied for its antibiotic, anti-inflammatory and anti-glycative properties ${ }^{29,30}$.

Our group has also shown the anti-inflammatory ability of MSX in an in vitro model of neurodegeneration with murine microglia and human neurons ${ }^{31}$. Herein, we sought to use MSX to modulate the pro-inflammatory effects of a western style diet in the brains of wild type C57BL/6 mice. 


\section{Materials and Methods}

\section{Animals and study conditions}

C57BL/6 mice were acquired from Jackson Labs (Bar Harbor, ME USA), acclimated for at least two weeks before being weight paired and housed four mice per cage. Mice were housed under 12 hour light/darkness cycles and were allowed to consume food and water ad libitum. Mice were fed either a standard grain free low fat diet (LFD) ( $n=8)$ (Research Diets Cat\# D12450B, New Brunswick, NJ), or a high fat diet where $45 \%$ of calories are derived from fat (HFD) (n=8) (Research Diets Cat\# D12451, New Brunswick, NJ. MSX was produced using food grade solvents and purification resins under cGMP conditions through Silicycle, Inc. (Québec, Canada) and was incorporated directly into LFD $(n=8)$ or HFD $(n=8)(0.5 \% \mathrm{w} / \mathrm{w})$. Body weights and food consumption were monitored weekly. Following 12 weeks of diet administration, all mice were anesthetized using isoflurane and sacrificed by cervical dislocation. Tissues were immediately harvested and snap frozen in liquid nitrogen. Brains were separated into cortex, cerebellum, and hippocampus regions for future analysis. This study and its protocols were approved by and conducted in accordance with the University of Rhode Island Institutional Animal Care and Use Committee (IACUC) (Approval \# AN09-07-004).

\section{Hepatic Lipid Isolation and Analysis}

Liver lipids from approximately $50 \mathrm{mg}$ of liver tissue were prepared using a chloroformmethanol extraction method described by More et $\mathrm{a}^{32}$. Triacylglyceride (TAG), Total Cholesterol, and Total Lipids were measured using specified kits from Wako Chemicals (Richmond VA USA).Total Non-esterified Free Fatty Acids (NEFA) were quantified using a kit from Pointe Scientific (Ann Arbor, MI USA). 


\section{Nucleic Acid Isolation from Tissue}

Whole hippocampi were isolated at the time of necropsy and snap frozen for nucleic acid isolation. Tissue was subsequently homogenized using Trizol and a dounce homogenizer. Homogenized material was then added directly to a Trizol Spin Column (Zymo Research, San Diego, CA USA) to isolate nucleic acids according to the manufacturer's protocol. RNA concentration and quality was then determined using a spectrophotometer (Nanodrop, Thermo Fisher, Waltham, MA USA)

\section{Gene Expression Analysis}

Genes involved in inflammatory, neurodegenerative, and antioxidant response processes were multiplexed from single RNA samples using a custom QuantiGene Luminex xMAP Gene Expression panel (Thermo Fisher, Waltham, MA USA). Analysis of the multiplex panel was performed according to the manufacturer's protocols with mean fluorescence intensity quantified using a Biorad Bioplex 200 instrument (Hercules, CA USA). Genes of interest include IL-1 $\alpha$, IL-1 $\beta$, IL-2, IL-3, IL-4, IL-6, IL-7, IL-9, IL-10, IL-11, IL-13, IL-15, IL-16, IL-18, IL-19, IFNa, IFN $\gamma$, TNF, CD38, CD70, FASL, MIF, CSF1, CD40LG, LEPR, CD36, MAPT, GSK3 3 , APP, PPAR $\gamma$, ITGAM, STING, NOS2, SOD1, and NFEL2.

\section{Statistical Analysis}

Body weight, Tissue weight and Hepatic lipid values are shown as mean \pm standard error (SEM). Gene expression data is shown as mean fluorescence intensity (MFI) \pm standard error. Data was analyzed using one way analysis of variance (ANOVA) where $p<0.05$ were statistically significant. Calculations were performed using Graphpad Prism (Graphpad Prism Software for Windows Ver 8.0, La Jolla, CA USA). 


\section{Results}

MSX attenuated HFD induced white adipose, liver and body weight increases

Body, liver, and white adipose tissue (WAT) weights at the time of necropsy were

significantly increased as a result of HFD. Body weight comparisons between LFD mice $(25.8 \mathrm{~g} \pm$

$0.92 \mathrm{~g})$ and HFD fed mice $(37.8 \mathrm{~g} \pm 1.37 \mathrm{~g})$ showed an increase of approximately $46.5 \%$

(Supplemental Figure 1A). Liver tissue showed a similar increase between LFD and HFD fed mice of approximately 50.0\% (Supplemental Figure 1B) but a 112.6\% increase in WAT weights were observed when comparing LFD fed mice to HFD fed mice(Supplemental Figure 1C). Interestingly, HFD+MSX mice had significantly lower body, liver, and WAT weights than HFD fed mice by $18.1 \%, 57.5 \%$, and $30.7 \%$ respectively.

\section{MSX modulates HFD associated increases in serum IL-6 and hepatic lipid accumulation and metabolism}

The HFD increased total lipid, non-esterified free fatty acid (NEFA) and triacylglyceride (TAG) content by $41.1,40.6$, and $54.3 \%$ respectively when compared to LFD control mice. Mice fed a HFD in combination with MSX had significantly lower hepatic cholesterol, total lipid, NEFA and TAG levels by approximately $40.1,24.5,51.0$, and $49.3 \%$ respectively as compared to HFD fed control mice (Supplemental Figure 2). MSX supplementation with LFD decreased hepatic cholesterol, total lipid, NEFA and TAG levels in LFD mice significantly by $45.5,34.7,51.6$, and $48.5 \%$ respectively compared to LFD control mice. Serum was also analyzed for proinflammatory marker, IL-6, and was found to be elevated in HFD mice $(29.4 \mathrm{pg} / \mathrm{ml} \pm 8.11)$ as compared to LFD $(21.8 \mathrm{pg} / \mathrm{ml} \pm 3.15$ (not statistically significant). The addition of MSX to LFD and HFD groups attenuated IL- 6 serum concentrations by 53.9 and $59.3 \%$ respectively compared to LFD and HFD mice. 
MSX significantly reduces the expression of Interleukins and inflammatory mediators in HFD hippocampi

HFD feeding for 12 weeks showed statistically significant increases in all interleukins (1, $2,3,4,6,7,9,10,11,13,15,16,18,19$ ) and interferons ( $\alpha$ and $\gamma$ ) as compared to LFD fed mice Figures 1 and 2. MSX at a dose of $0.5 \% \mathrm{w} / \mathrm{w}$ was evaluated for its ability to modulate the expression of these inflammatory mediators. HFD+MSX mice demonstrated significant modulation of IL-7, TNF $\alpha$, IL-11, IL-19, and IFN $\alpha$ were significantly decreased by $71.4 \%, 55.5 \%$, $34.4 \%, 18.6 \%$ and $79.8 \%$ respectively as compared to HFD mice. Several other cytokines showed decreasing trends but were not statistically significant. Interestingly, IL-13 and IL-16 were upregulated by $74.5 \%$ and $97.7 \%$ respectively when comparing HFD to HFD+MSX. LFD+MSX mice showed significantly increased expression in IL-1 $\alpha$, IL-1 $\beta$, IL-2, IL-3, IL4, IL-9, IL-13, IL-16, and IL-19.

Gene expression of anti-oxidative enzymes and AD-associated enzymes are differentially expressed in both HFD and MSX supplemented HFD mice

Prolonged exposure of mice to HFD led to the elevation of three key AD-associated genes: MAPT (i.e. Tau), GSK3 $\beta$, and APP by 1.32, 1.31 and 1.29 fold increase over LFD respectively (Figure 3 ). It is worth noting that APP, the processing protein responsible for cleaving and releasing Beta Amyloid in the brain, was down regulated with the addition of MSX by $13.9 \%$ however, the trend was not statistically significant $(p=0.1894)$.

Nitric Oxide Synthase 2 (NOS2), a pro and anti-inflammatory protein, was overexpressed in HFD mice by $76.6 \%$ as compared to LFD mice. Moreover, NOS2 was significantly upregulated by $74.5 \%$ in MSX supplemented HFD mice (Figure 3 ) as compared to the HFD control mice. 
Antioxidant response genes, Super Oxide Dismutase 1 (SOD1) and Nuclear factor (erythroid-derived 2)-like 2(NFEL2) commonly known as NRF2, were significantly upregulated by $39.7 \%$ and $61.1 \%$ respectively in HFD fed mice as compared to LFD controls. MSX supplemented with HFD reduced the expression of SOD1 (28.0\%) and NRF2 (38.0\%) as compared to mice fed a HFD. PPARY, ITGAM and STING were also upregulated in HFD fed mice as compared to LFD fed counterparts by $53.7 \%, 62.5 \%$, and $40.6 \%$ respectively. HFD+MSX mice showed reductions in the expression of the macrophage-associated gene, ITGAM, by approximately $25.4 \%$.

\section{HFD augments TNF family ligands and Fatty Acid Transport Protein Genes}

CD38, CD70, FAS ligand, CD40 ligand are part of the TNF family and thus part of the proinflammatory response in diet-induced obesity ${ }^{33-35}$. HFD fed mice demonstrated significant increases in the expression of these mediators by $135.3 \%, 288.4 \%, 184.7 \%$, and $88.6 \%$ respectively as compared to LFD control mice (Figure 4). MSX added to HFD reduced expression of only CD40LG and CD70 significantly as compared to HFD mice by approximately, $59.9 \%$ and $35.2 \%$. HFD mice were found to have significantly upregulated expression levels of Macrophage/Microglial associated genes, Macrophage Infiltration Factor (MIF) and Colony Stimulating Factor 1 (CSF1) by $46.4 \%$ and $74.8 \%$. 
MSX supplementation to LFD increased the hippocampal gene expression of both MIF and CSF1 by $21.2 \%$ and $117.2 \%$ respectively. Leptin Receptor and CD36, two major Fatty Acid Transport associated genes, were also markedly increased in High Fat Diet fed mice by $192.1 \%$ and $82.8 \%$ respectively. MSX supplementation in HFD mice reduced expression of LEPR and CD36 by $76.2 \%$ and $7.4 \%$ respectively as compared to HFD control mice. HFD+MSX mice LEPR expression levels were found to be similar to LFD control mice $(7.13 \mathrm{MFI} \pm 0.94)$ and even the addition of MSX to the LFD mice showed further decreased levels of LEPR expression 6.075 MFI \pm 0.75 (14.9\% reduction). CD36, however, showed a non-statistically significant decreasing trend in both LFD and HFD groups supplemented with MSX.

\section{Discussion}

Wild type mice were used to model the effects of diet modification on various metabolic and immunological endpoints given that diet-induced obesity may lead to chronic inflammation and is closely involved in a variety of disease states including Non-alcoholic steatohepatitis (NASH), type II diabetes mellitus (T2DM), Neurodegenerative diseases (Alzheimer's, Parkinson's and Amyotrophic Lateral Sclerosis), and even cancer ${ }^{5,36-38}$. In the case of neurodegenerative diseases, studies have shown that specific dietary components may contribute to the weakening of the blood brain barrier and ultimately the progressive activation of microglia ${ }^{39,40}$. Activation and further macrophage recruitment, is inherently a response to rid the tissue of foreign infection and/or damaged cells but over time, the signaling capacity of microglia to respond to pathogen associated molecules (i.e. lipopolysaccharide or bacterial DNA) or damage associated molecules (i.e. nucleic acids from necrotic/apoptotic cells) weakens and thus to compensate, more macrophages are recruited and activated ${ }^{11,41}$. 
This flood of inflammatory stimuli leads to the necrosis and apoptosis of neurons and depending on the localization of this degradation, manifests into AD, PD, or ALS.

Diet as a means of intervention in these initial inflammatory processes, are largely due in part to anti-oxidant activity of food sources ${ }^{26,42}$. MSX, a food grade phenol-enriched extract derived from maple syrup, represents a chemically unique and under-researched product with over 60 individual chemical constituents including lignans, phenolic acids, coumarins, and stilbenes $^{28}$. In this study, mice were subjected to a diet rich in saturated fats (45\%kcal derived from fat) for approximately 12 weeks. Final body weights were significantly increased in HFD fed mice as compared to LFD control mice, MSX supplementation to HFD afforded a significant reduction in body weight, liver weight, and white adipose tissue. Moreover, MSX incorporation in both HFD and LFD showed significantly reduced concentrations of IL-6 in serum (Supplemental Figure 3). Hepatic lipids, free fatty acids, and triacylglyceride levels which were elevated in HFD mice, were abrogated with MSX supplementation. Given the presence of metabolic syndrome/NASH indicators, further investigation into the effects of diet and neuroinflammation were conducted. 
In this in vivo model of diet-induced obesity, we report, MSX incorporation into feed afforded a statistically significant reduction in the gene expression of key inflammatory interleukins, interferons and even some major receptors associated with fatty acid transport and cell apoptosis. Notably, IL-7 which is heavily involved in B-cell proliferation, T-cell survival, and stimulation of interleukins in monocytes, was augmented in HFD but abrogated in HFD+MSX mice ${ }^{43,44}$. IL-11 is responsible for protecting epithelial and connective tissue, stimulating neuronal development and serves as a growth factor for erythroid and myeloid progenitor cells, was upregulated in HFD mice but significantly attenuated in LFD+MSX and HFD+MSX mice ${ }^{45}$. Similarly, IL-19 which induces Th2 cytokine release in monocytes and macrophages, was increased in HFD but decreased in MSX supplemented HFD mice. Interferon $\alpha$, was significantly increased in HFD fed mice but was significantly abrogated to levels found in LFD controls. TNF $\alpha$, an essential cytokine, growth factor, and intercellular signaling molecule, which is seen largely as a pro-inflammatory molecule was also significantly overexpressed in HFD fed mice but also attenuated to levels at or below LFD control fed mice ${ }^{46}$.

AD-related protein, Amyloid Precursor Protein (APP), was significantly elevated in HFD fed mice as compared to LFD controls. However, MSX supplementation in LFD or HFD diets decreased expression ( $p$ value of 0.1894 (HFD vs. HFD+MSX)). Interestingly, Tau and APP were significantly upregulated, consistent with findings from other long term diet-induced obesity rodent studies ${ }^{16,47,48}$. Although, the addition of MSX did not abrogate these markers, it is possible that effects of MSX were diminished or masked after such prolonged diet exposure. ITGAM or Integrin Alpha Subunit M which is commonly known as Macrophage Receptor 1 (MAC1) mediates complement coated particles for macrophage phagocytosis and degradation ${ }^{49,50}$. ITGAM/MAC-1 expression was significantly increased in HFD mice but also modulated in MSX supplemented HFD mice. 
Another TNF regulated molecule that was modulated by MSX, was CD40 ligand (CD40LG). CD40LG is responsible for the stimulation and growth of T-cell populations while also promoting the release of IL-4 and IL-10 51 . The death receptor ligand, FASL, which was upregulated in HFD fed mice was also significantly attenuated in HFD + MSX, fed mice. Taken together, the exposure of MSX to mice appears to modulate TNF $\alpha$ and immune response pathways mediated by TNF.

Finally, a fatty acid transport protein CD36 (aka Fatty Acid Translocase, FAT) and Leptin Receptor were markedly expressed in HFD mice as compared to their LFD controls. MSX decreased CD36 expression slightly in HFD fed mice ( $p$ value $=0.5548)$ however LEPR was significantly decreased in both LFD and HFD fed mice supplemented with MSX as compared to LFD fed control mice. As expected, leptin receptor expression is increased in mice with an obese, glucose/insulin intolerant phenotype ${ }^{52}$. Typically, LEPR signaling is not only associated with food reward response but is implicated in neuronal plasticity and is needed to support cognitive function ${ }^{53,54}$. While circulating levels of leptin were not measured in this study, low leptin levels in the brain (particularly hypothalamus) signal for increase food intake $e^{54,55}$. Reducing LEPR expression in cell specific or key areas of the brain however, may actually reduce food intake and be involved with long term weight loss $s^{55,56}$. In the hippocampus, neuroinflammatory and thus neurodegenerative processes are heavily influenced by leptin signaling disruption and the result of obesity ${ }^{57}$. It has been proposed that neuronal leptin resistance in $A D$ is due to hyperphosphorylation of microtubule associated protein tau (MAPT, tau) by GSK3 $\beta$ and aggregation of neurofibrillary tangles (NFTs) ${ }^{56,57}$. 
These events prevent neuronal LEPR from binding to leptin signaling which increases gene expression of LEPR and thus increases the biosynthesis of leptin. Hippocampal neurons which are hyposensitive to leptin causes cognitive deficits by pre-mature cell death via leptin hypersensitive astrocyte/microglia cytokine secretion or "reactive astrocytosis/microgliosis" 56 . While this current study shows that MAPT and GSK3 $\beta$ levels were significantly increased in HFD only fed mice, the addition of MSX to the HFD did not modulate gene expression. Considerable studies are needed to support this observation that weight loss and a decrease in neuroinflammation in HFD+MSX mice is attributable to modulation in the leptin signaling cascade.

In the present study, MSX was shown to elevate the expression of a number cytokines such as IL-1 ( $\alpha$ and $\beta$ ) IL-2, 3, 4, 9, 13, 15, 16, 18, and 19 as compared to LFD alone and even some HFD fed mice. Moreover, some LFD+MSX mice also displayed elevated levels of FASL, CD38, CD70, CSF1, and CD36. The gene expression of these factors are varied throughout the brain and during distinct ages. For example, IL-1 $\beta$, which is regarded as a pro-inflammatory signal, is actually needed to maintain neuronal synaptic plasticity ${ }^{58}$. The increase in the expression of the interleukins and cellular factors could be a compensatory mechanism or stimulatory response to MSX. Despite gene expression being elevated, MSX may influence specific transcription factors which may only be confirmed at the proteomic level. These cytokines, as outlined in Table 1, stimulate the proliferation and differentiation of regulatory Tcells (IL-2), basophils, eosinophils (IL-3, 4, 13, 16), CD8 T-cells (IL-9, 16), B-cells (IL-13), NKT cells (IL-15). IL-18 and IL-19 are associated with inducing Th1/Th2 or inducing Th2 cytokine production respectively ${ }^{59}$. 
The recruitment or infiltration of immune cells are seen as a necessary response to degrade, process, and transport necrotic/apoptotic cells and cellular debris (fibrils, plaques etc. $)^{41,60}$. Recruitment of phagocytic macrophages as evidenced by CSF1 expression may be indicative of beneficial macrophages clearing debris. That being said, a long term diet-induced obesity study is needed to histologically confirm this in vivo observation that MSX induces key microglia associated and inflammatory genes to promote the clearance of damaged cells, cellular debris, and pathogenic substances.

The Luminex xMAP platform was used to simultaneously quantify gene expression from isolated RNA in these tissues. The platform utilizes analyte-specific magnetic bead coated probes, which, when bound and exposed to red and near infrared laser sources, will fluoresce at specific intensities. These beads are assigned a unique bead number and region which allows for the differentiation of each bead at these various intensities. Normalization to a housekeeper gene is not necessary, as greater specificity can be obtained with each probe design unlike qRT$\mathrm{PCR}^{61-63}$. A limitation in this study is the inherent biological variability mouse to mouse ${ }^{64}$. Housing conditions of these mice were 4 mice per group and given the implications of gut microbial transformation of these phytochemicals from MSX, cohousing effects, individual food consumption differences and overall cage to cage variability may have played a role in not only what constituents make it to the brain but at what concentration.

The extent of Blood Brain Barrier penetrability has not been determined with MSX. Brains of the mice in this study were not perfused but given that MSX is a complex extract, it is difficult to select one component of the extract as the sole source of the beneficial effects. 
One major phytochemical class of MSX, lignans, consists of molecules such as Secoisolariciresinol (SECO). In humans and rodents, SECO is converted to Enterodiol(ED) and

Enterolactone (EL) by the microbes of the gut ${ }^{65-67}$. EL in particular has been found to be brain penetrable in silico and using passive permeability assays (PAMPA) (data not shown).

\section{Conclusions}

Using a diet-induced obesity rodent model, we sought to examine the potential mechanisms associated with MSX and obesity associated neuroinflammation. MSX was able to modulate several key inflammatory molecules including IL-7, IL-10, TNF, FASL, and LEPR. Interestingly several potential pathways could be involved in the beneficial effects derived from MSX consumption including the macrophage recruitment/stimulation, TNF-family death receptor mediated apoptosis, and leptin signaling. Further in vitro and in vivo studies are warranted to fully explore MSX at various doses in these systems and pathways in addition to the identification of representative compounds or metabolites from each major phytochemical class responsible for these biological effects. 


\section{Funding Acknowledgements}

This study was supported through a University of Rhode Island Council for Research Grant and the Quebec Maple Syrup Producers (QMSP).

\section{Author Contributions}

NAD wrote the manuscript, designed, conducted, and analyzed the gene expression experiments. MP, EM, and HM oversaw animal care and feeding. MP and EM designed, conducted and analyzed metabolic endpoint experiments during and concluding the animal study. AS and NPS conceived the overall study and edited the manuscript.

\section{Conflict of Interest}

The authors of this manuscript declare no financial conflict of interest 
Figures
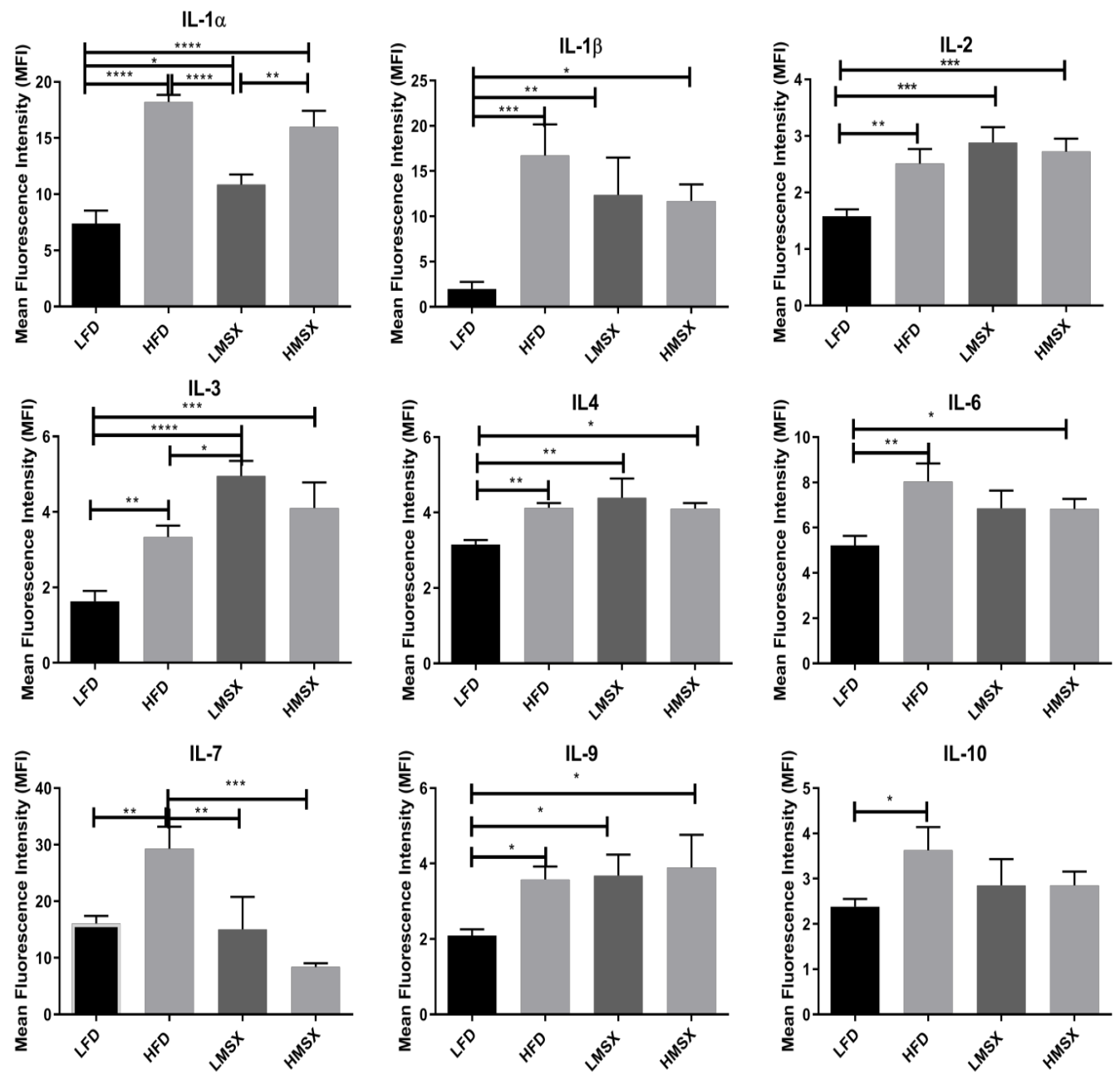

Figure 1.) Effects of High Fat Diet and High Fat Diet with MSX show a general reduction in proinflammatory Interleukins. 12 Week HFD feeding afforded a significant increase in proinflammatory cytokines, interferons, and growth factors in response to damage and pathogen derived signals. MSX in a HFD abrogated key migratory and immune cell differentiation markers such as IL-6 (n.s), IL-7, and IL-10 (n.s) as compared to HFD controls. Statistical significance was calculated using one way ANOVA where $p<0.05^{*}, p<0.01^{* *}, p<0.001^{* * *}, p<0.0001^{* * * *}$, or not statistically significant (n.s). 

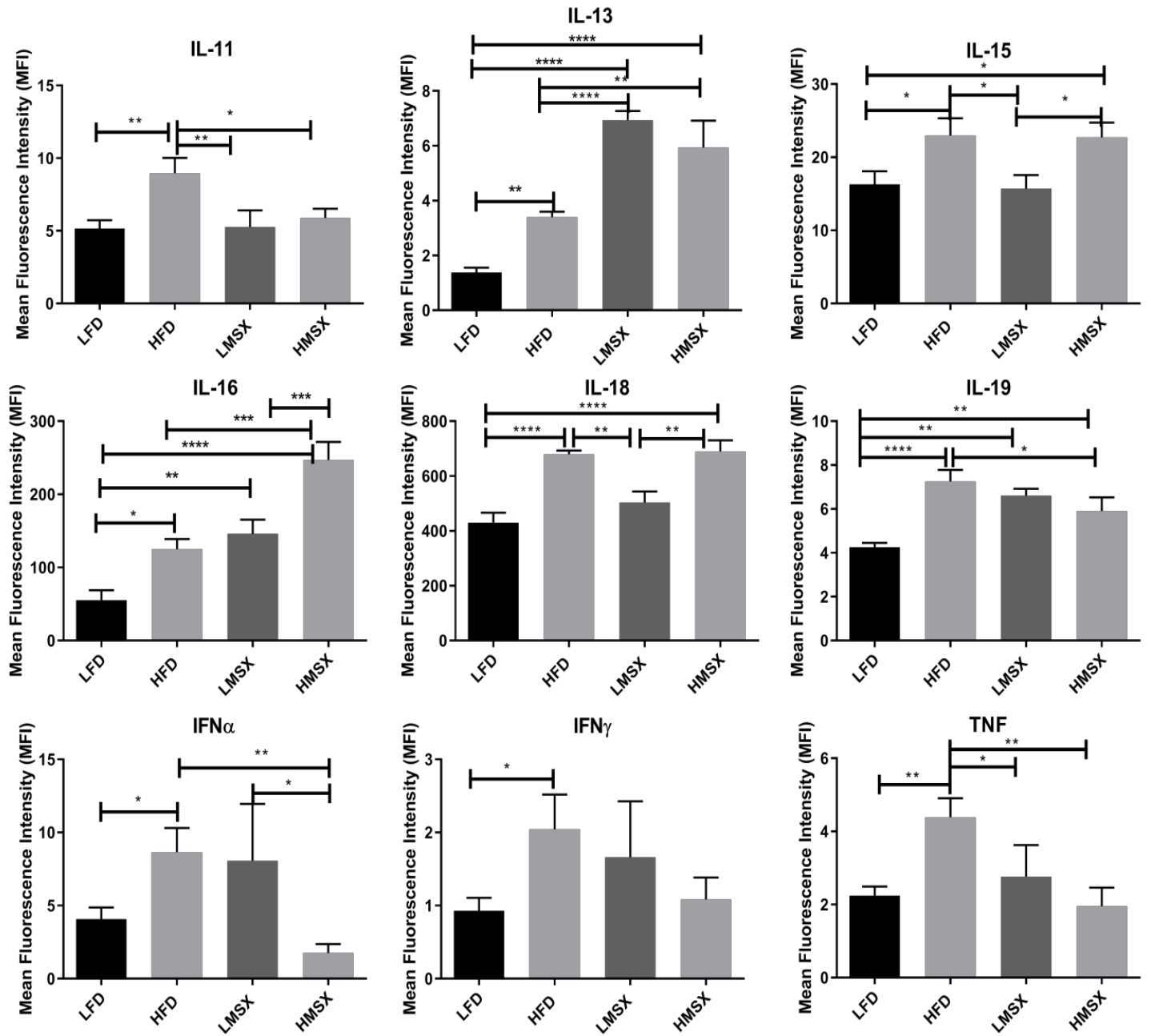

Figure 2.) MSX modulates pro-inflammatory effects of High Fat Diet consumption. 12 Week HFD feeding afforded a significant increase in pro-inflammatory cytokines, interferons, and growth factors in response to damage and pathogen derived signals. MSX in HFD abrogated IL11, IL-19, IFNa, IFNY (n.s), and TNF gene expression as compared to HFD controls. Statistical significance was calculated using one way ANOVA where $p<0.05^{*}, p<0.01 * *, p<0.001^{* * *}$, $p<0.0001^{* * * *}$, or not statistically significant (n.s). 

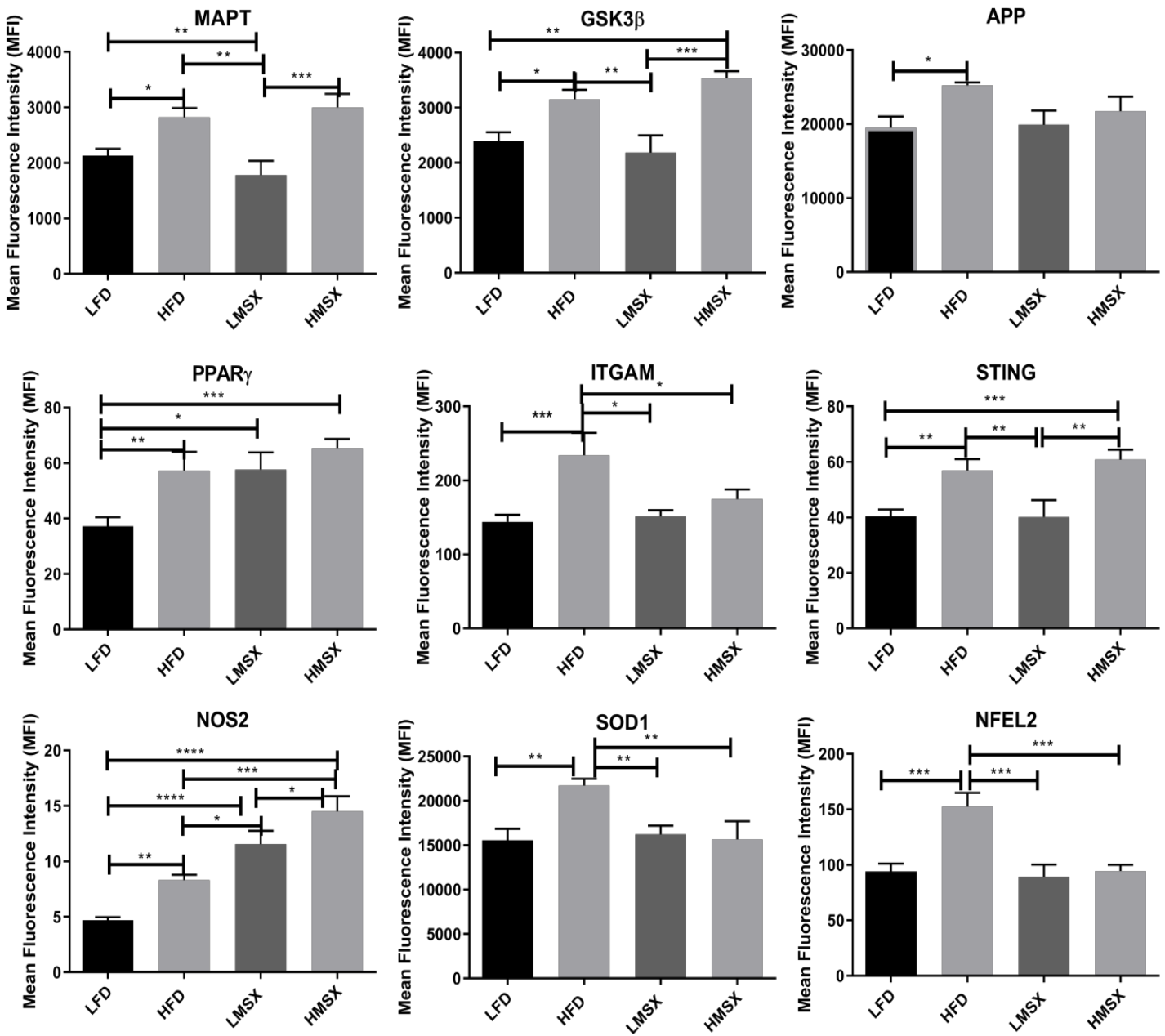

Figure 3.) High Fat Diet Fed Mice show increased levels of Alzheimer's disease related genes and compensatory mechanism associated anti-oxidant response genes. As seen is previous diet-induced obesity studies, a HFD caused an increase in the expression of neurodegenerative processing proteins (MAPT, GSK3B, and APP) while also stimulating macrophage migratory and inflammatory gene signaling (ITGAM, STING) and increasing antioxidant response genes (SOD1, and NFEL2). MSX supplementation in HFD feed caused a reduction in APP (n.s), ITGAM, SOD1 and NFEL2. Statistical significance was calculated using one way ANOVA where $p<0.05^{*}, p<0.01 * *, p<0.001^{* * *}, p<0.0001^{* * * *}$, or not statistically significant (n.s). 

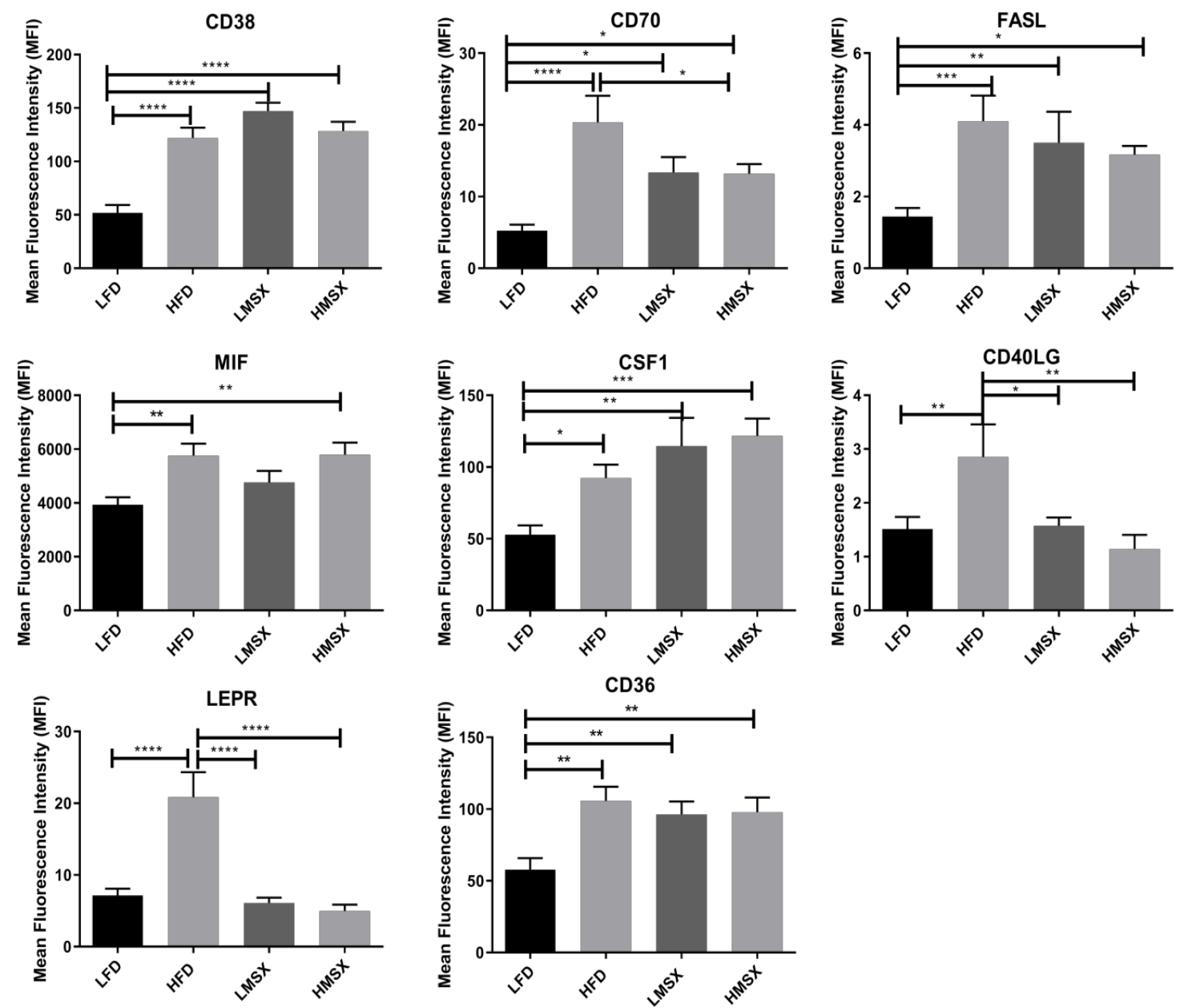

Figure 4.) MSX modulates gene expression of Macrophage and Metabolic Syndrome

associated ligands and receptor genes. Mice fed a HFD showed increased macrophage (CD38, MIF, and CSF1) and death associated markers (FASL, CD4OL) including up-regulation of fatty acid transport/metabolism associated genes (CD36, LEPR). The addition of MSX significantly reduced CD40LG, CD70 and LEPR. Statistical significance was calculated using one way ANOVA where $p<$ $0.05^{*}, \mathrm{p}<0.01^{* *}, \mathrm{p}<0.001^{* * *}, \mathrm{p}<0.0001^{* * * *}$, or not statistically significant (n.s). 
Table 1.) Gene Multiplex Targets, Functions and Statistical Comparisons

\begin{tabular}{|c|c|c|c|c|c|}
\hline GENE & FUNCTION $^{15,33-35,47,49,51,57,59,68-74}$ & $\begin{array}{l}\text { HFD VS } \\
\text { LFD } \\
\text { (MEAN } \\
\text { DIFF) }\end{array}$ & P VALUE & $\begin{array}{l}\text { HMSX VS } \\
\text { HFD } \\
\text { (MEAN } \\
\text { DIFF) } \\
\end{array}$ & P VALUE \\
\hline IL-1 $\alpha$ & \multirow{2}{*}{$\begin{array}{c}\text { Induce pro-inflammatory } \\
\text { cytokines and differentiate } \\
\text { Th17 cells }\end{array}$} & 10.81 & $<0.0001$ & -2.217 & 0.1677 \\
\hline IL-1 $\beta$ & & 14.72 & 0.0007 & -5.01 & 0.2165 \\
\hline IL-2 & $\begin{array}{c}\text { Stimulate Tregs, T and B } \\
\text { effectors, and NKTs }\end{array}$ & 0.9238 & 0.004 & 0.2143 & 0.4941 \\
\hline IL-3 & $\begin{array}{c}\text { Activate basophil and } \\
\text { eosinophils, recruit phagocytic } \\
\text { cells }\end{array}$ & 1.708 & 0.0027 & 0.7625 & 0.2032 \\
\hline IL-4 & $\begin{array}{l}\text { Induce Th2 cells, induce tissue } \\
\text { adhesion and inflammation }\end{array}$ & 0.975 & 0.0078 & -0.025 & 0.9489 \\
\hline IL-6 & $\begin{array}{l}\text { Infiltration of leukocytes, T-cell } \\
\text { and B-cell differentiation, } \\
\text { survival of cholinergic neurons }\end{array}$ & 2.823 & 0.0014 & -1.217 & 0.1734 \\
\hline IL-7 & $\begin{array}{c}\text { Proliferation of B-cells (pre and } \\
\text { pro), T-cell survival, induce } \\
\text { Interleukin synthesis in } \\
\text { monocytes }\end{array}$ & 13.22 & 0.0044 & -20.92 & 0.0001 \\
\hline IL-9 & $\begin{array}{l}\text { Growth factor for T and Mast } \\
\text { Cells, Inhibits Th1 cytokines } \\
\text { from CD8 T-cells }\end{array}$ & 1.481 & 0.0368 & 0.3179 & 0.6609 \\
\hline IL-10 & $\begin{array}{c}\text { Immunosupression via APCs or } \\
\text { T-cells }\end{array}$ & 1.245 & 0.0228 & -0.775 & 0.1972 \\
\hline IL-11 & $\begin{array}{l}\text { Growth factor for erythroid, } \\
\text { myeloid and megakaryocyte } \\
\text { progenitors. Protect epithelial } \\
\text { and connective tissue. Inhibts } \\
\text { monocyte and macrophage } \\
\text { activity. Neuronal development }\end{array}$ & 3.81 & 0.0018 & -3.083 & 0.0176 \\
\hline IL-13 & $\begin{array}{l}\text { Eosinophil and mast cell } \\
\text { recruitment, activation and } \\
\text { growth. MHC II upregulation on } \\
\text { B cells }\end{array}$ & 2.02 & 0.0073 & 2.533 & 0.0022 \\
\hline IL-15 & $\begin{array}{c}\text { T-cell proliferation and } \\
\text { activation (NKT), maintain CD8 } \\
\text { memory antispoptosis }\end{array}$ & 6.67 & 0.0215 & -0.2405 & 0.9383 \\
\hline IL-16 & $\begin{array}{l}\text { Major chemotractant for } \\
\text { CD4/CD8 T cells, monocytes, } \\
\text { mast cells and eosinophils }\end{array}$ & 69.56 & 0.0112 & 121.9 & 0.0002 \\
\hline IL-18 & $\begin{array}{c}\text { Induces IFNg with IL-12, } \\
\text { activates Th1 or Th2 }\end{array}$ & 249.00 & $<0.0001$ & 10.25 & 0.8489 \\
\hline IL-19 & $\begin{array}{c}\text { Induce Th2 cytokines, IL-6, TNFa } \\
\text { and IL-10 in monocytes }\end{array}$ & 3.00 & $<0.0001$ & -1.35 & 0.0415 \\
\hline IFN $\alpha$ & $\begin{array}{l}\text { Viral Response via adaptive } \\
\text { immune signaling, stimulate } \\
\text { DCs antigen presentation, } \\
\text { macrophage Promote naïve T- }\end{array}$ & 4.572 & 0.037 & -6.9 & 0.0058 \\
\hline
\end{tabular}




\begin{tabular}{|c|c|c|c|c|c|}
\hline GENE & FUNCTION $^{15,33-35,47,49,51,57,59,68-74}$ & $\begin{array}{l}\text { HFD VS } \\
\text { LFD } \\
\text { (MEAN } \\
\text { DIFF) }\end{array}$ & P VALUE & $\begin{array}{l}\text { HMSX VS } \\
\text { HFD } \\
\text { (MEAN } \\
\text { DIFF) }\end{array}$ & P VALUE \\
\hline & $\begin{array}{l}\text { cell growth and tumor /virus } \\
\text { cell apoptosis }\end{array}$ & & & & \\
\hline IFNY & $\begin{array}{l}\text { Th1 cell cytotoxic response and } \\
\text { differentiation, upregulates } \\
\text { MHC I and II expression, inhibts } \\
\text { cell growth (epithelial), } \\
\text { proapoptosis/cell cycle } \\
\text { modulation }\end{array}$ & 1.113 & 0.0426 & -0.9595 & 0.1151 \\
\hline TNF & $\begin{array}{c}\text { Pro-inflammatory and } \\
\text { immunosuppressive by } \\
\text { initiating or limiting severity } \\
\text { and duration of inflammatory } \\
\text { response }\end{array}$ & 2.141 & 0.0033 & -2.436 & 0.0026 \\
\hline MAPT & $\begin{array}{l}\text { aka Protein Tau, leads to the } \\
\text { generation of Tau tangles } \\
\text { (immune reactive peptides) }\end{array}$ & 692.7 & 0.0263 & 175 & 0.567 \\
\hline GSK3 $\beta$ & $\begin{array}{l}\text { Neuronal development via } \\
\text { neuronal progenitor } \\
\text { differentiation }\end{array}$ & 751.8 & 0.0301 & 387.8 & 0.2624 \\
\hline APP & $\begin{array}{l}\text { Amyloid Precursor protein, } \\
\text { normally involved in neuronal } \\
\text { plasticity \& development. Over } \\
\text { expression leads to plaque } \\
\text { formation and microglial } \\
\text { activation }\end{array}$ & 5721 & 0.0488 & -3497 & 0.1894 \\
\hline PPARY & $\begin{array}{l}\text { Neuronal depression of } \\
\text { inflammation associated with } \\
\text { chronic or acute insult. Glucose } \\
\text { absorption, lipid balance and } \\
\text { cell growth/differentiation }\end{array}$ & 20 & 0.0097 & 8.188 & 0.2769 \\
\hline ITGAM & $\begin{array}{c}\text { Leukocyte adhesion, aka } \\
\text { Macrophage Receptor } 1 \text {, } \\
\text { upregulated by TNF, mediate } \\
\text { complement coated } \\
\text { macrophage uptake of particles } \\
\text { for degradation }\end{array}$ & 90.02 & 0.0009 & -59.6 & 0.0214 \\
\hline STING & $\begin{array}{l}\text { Stimulates Interferon Genes in } \\
\text { response to viral or internal } \\
\text { pathogens (microbial DNA). } \\
\text { Localizes with autophagy } \\
\text { related proteins }\end{array}$ & 16.42 & 0.0031 & 3.964 & 0.478 \\
\hline NOS2 & $\begin{array}{l}\text { Induced by IFN } \gamma, \text { TNF and IL-1B. } \\
\text { Macrophage associated NO } \\
\text { production, stimulates IL-6 and } \\
\text { IL-8, stimulate epithelial cell } \\
\text { growth }\end{array}$ & 3.604 & 0.0066 & 6.19 & 0.0001 \\
\hline
\end{tabular}




\begin{tabular}{|c|c|c|c|c|c|}
\hline GENE & FUNCTION $^{15,33-35,47,49,51,57,59,68-74}$ & $\begin{array}{l}\text { HFD VS } \\
\text { LFD } \\
\text { (MEAN } \\
\text { DIFF) } \\
\end{array}$ & P VALUE & $\begin{array}{l}\text { HMSX VS } \\
\text { HFD } \\
\text { (MEAN } \\
\text { DIFF) }\end{array}$ & P VALUE \\
\hline SOD1 & $\begin{array}{l}\text { Anti-oxidant response by } \\
\text { binding to superoxide radial } \\
\text { molecules }\end{array}$ & 6170 & 0.0019 & -6077 & 0.004 \\
\hline NFEL2 & $\begin{array}{l}\text { aka NRF2, interacts with anti- } \\
\text { oxidant response element, } \\
\text { stimulating Phase II anti- } \\
\text { oxidant response enzymes }\end{array}$ & 58.44 & 0.0002 & -58.02 & 0.0003 \\
\hline CD38 & $\begin{array}{c}\text { TNF } \alpha \text { inducible, Dendritic } \\
\text { organization in hippocampal } \\
\text { excitatory neurons, humoral } \\
\text { immune response }\end{array}$ & 70.13 & $<0.0001$ & 6.503 & 0.6136 \\
\hline CD70 & $\begin{array}{l}\text { TNF ligand, activates T and B } \\
\text { cells, improves cytotoxic } \\
\text { function of NK cells, } \\
\text { proliferation of CD8 T cells }\end{array}$ & 15.11 & $<0.0001$ & -7.167 & 0.0372 \\
\hline FASL & $\begin{array}{l}\text { TNF ligand, forms Death } \\
\text { Domain Complex leading to } \\
\text { caspase } 8 \text { apoptosis }\end{array}$ & 2.66 & 0.0003 & -0.9333 & 0.1914 \\
\hline MIF & $\begin{array}{c}\text { Macrophage Migration } \\
\text { Inhibitor Factor, response to } \\
\text { bacterial infection (LPS or } \\
\text { DNA), stimulates cytokine } \\
\text { release in macrophages }\end{array}$ & 1824 & 0.0038 & 38.59 & 0.9472 \\
\hline CSF1 & $\begin{array}{c}\text { Colony Stimulating Factor -1 } \\
\text { induces macrophage } \\
\text { infiltration, induces pro- } \\
\text { inflammatory cytokine } \\
\text { secretion }\end{array}$ & 39.53 & 0.0429 & 29.44 & 0.1134 \\
\hline CD40LG & $\begin{array}{l}\text { TNF ligand, stimulate T-cell } \\
\text { growth and cytokine synthesis } \\
\text { (IL-4 and IL-10) }\end{array}$ & 1.339 & 0.0071 & -1.707 & 0.0016 \\
\hline LEPR & $\begin{array}{l}\text { Neuronal stimulation and } \\
\text { plasticity, food reward (anti- } \\
\text { inflammatory).Pro- } \\
\text { inflammatory and increased } \\
\text { with HFD or metabolic } \\
\text { syndrome }\end{array}$ & 13.71 & $<0.0001$ & -15.88 & $<0.0001$ \\
\hline CD36 & $\begin{array}{l}\text { aka FAT, transport fatty acids } \\
\text { into and out of wide range of } \\
\text { cells. Inhibited by LPS and HDL, } \\
\text { induced by cholesterol, CSF, IL- } \\
4 \text {, insulin and glucose. } \\
\text { Controlled by PPARY in } \\
\text { adipocytes }\end{array}$ & 47.9 & 0.0013 & -7.857 & 0.5548 \\
\hline
\end{tabular}


A

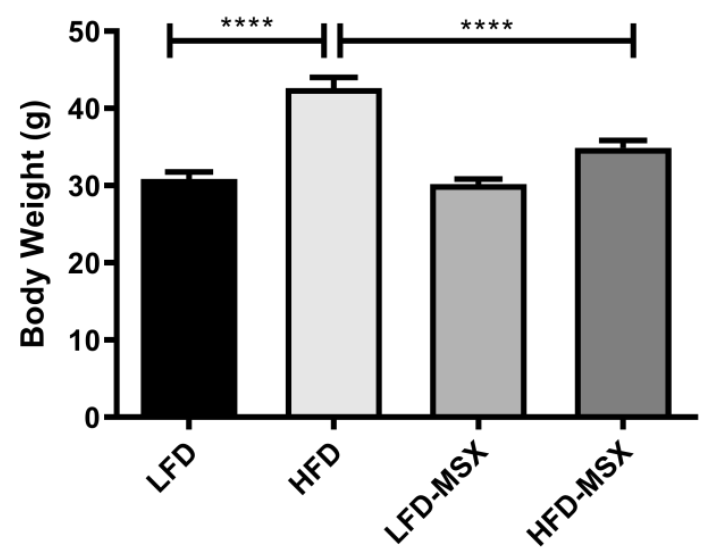

B

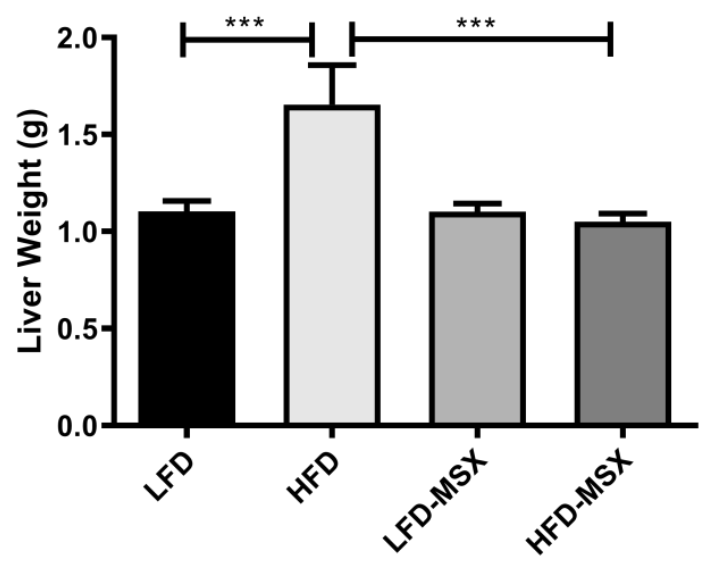

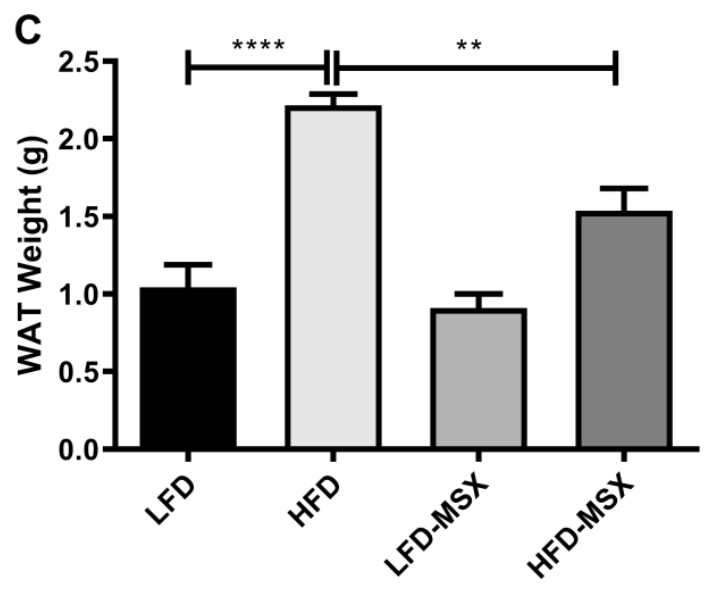

Supplemental Figure 1.) Final Body and Tissue Weights after 12 Weeks of High Fat Diet exposure with or without MSX supplementation. Final tissue and body weights were significantly elevated through HFD feeding as compared to LFD mice. MSX incorporation into a HFD prevented these weight increases. Statistical significance was calculated using one way ANOVA where $\mathrm{p}<0.05^{*}, \mathrm{p}<0.01^{* *}, \mathrm{p}<0.001^{* * *}, \mathrm{p}<0.0001^{* * * *}$, or not statistically significant (n.s). 
A
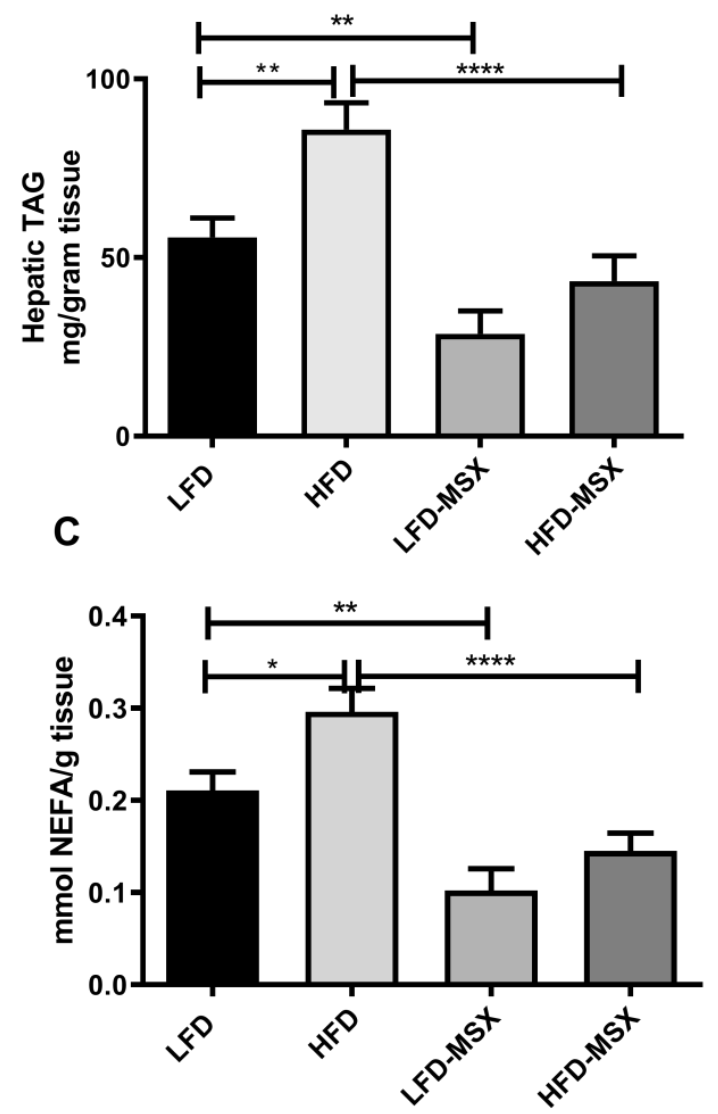

B
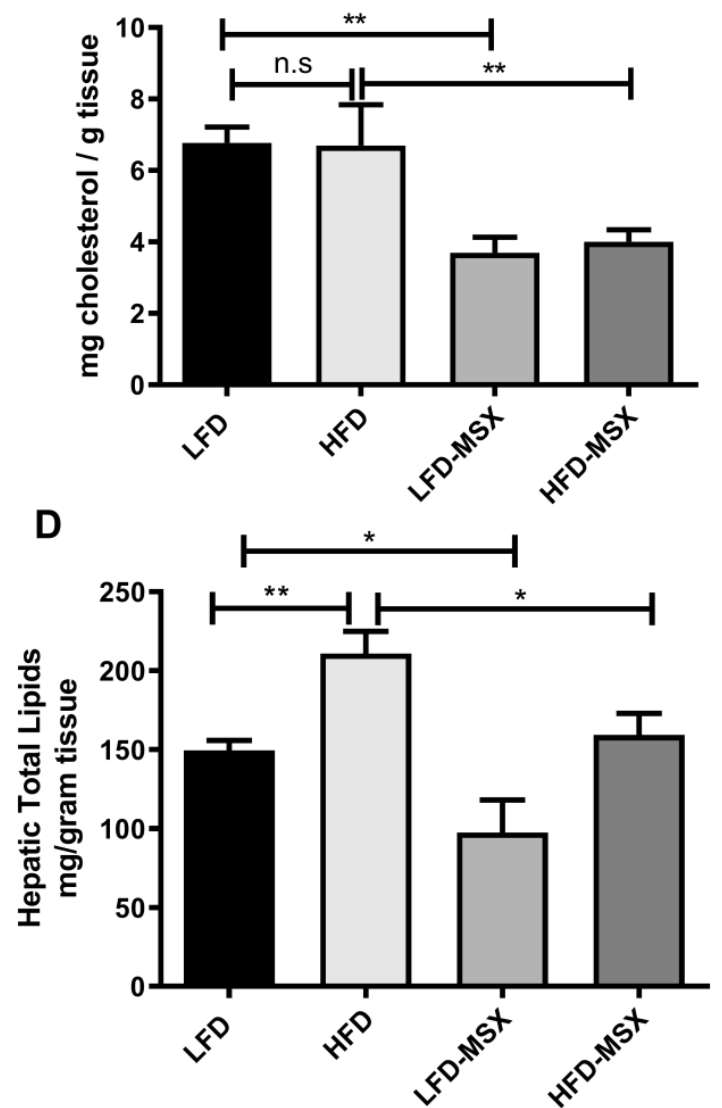

Supplemental Figure 2.) Hepatic Lipid Composition of High Fat Diet fed mice with and without MSX. Liver lipid transport and metabolism markers were significantly elevated (less cholesterol) as compared to LFD control mice. MSX supplementation caused a significant reduction in TAG , NEFA, Total lipids and cholesterol as compared to HFD controls. Statistical significance was calculated using one way ANOVA where $p<0.05^{*}, p<0.01^{* *}, p<0.001^{* * *}, p<0.0001^{* * * *}$, or not statistically significant (n.s). 


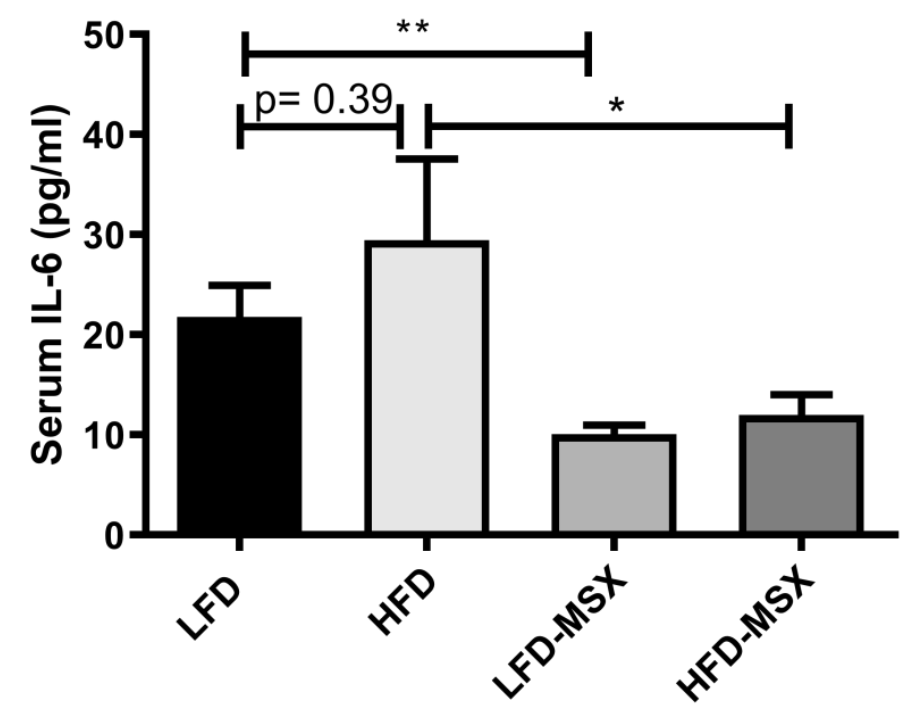

Supplemental Figure 3.) Serum IL-6 concentrations are abrogated with the addition of MSX in Low Fat and High Fat Diets. The pro-inflammatory effects of a HFD were analyzed through ELISA and found to be significantly elevated as compared to LFD control mice. HFD mice supplemented with MSX saw a significant attenuation in serum IL-6 concentration. Statistical significance was calculated using one way ANOVA where $p<0.05^{*}, p<0.01 * *, p<0.001^{* * *}$, $\mathrm{p}<0.0001^{* * * *}$, or not statistically significant (n.s). 


\section{References and Works Cited}

1. Glaros, T. G. et al. Causes and consequences of low grade endotoxemia and inflammatory diseases. Front. Biosci. Sch. Ed. 5, 754-765 (2013).

2. Wolowczuk, I. Obesity - an inflammatory state. Acta Vet. Scand. 57, K5 (2015).

3. Boutagy, N. E., McMillan, R. P., Frisard, M. I. \& Hulver, M. W. Metabolic endotoxemia with obesity: Is it real and is it relevant? Biochimie 124, 11-20 (2016).

4. Faraj, T. A., McLaughlin, C. L. \& Erridge, C. Host defenses against metabolic endotoxaemia and their impact on lipopolysaccharide detection. Int. Rev. Immunol. 36, 125-144 (2017).

5. Moreira, A. P. B., Texeira, T. F. S., Ferreira, A. B., Peluzio, M. do C. G. \& Alfenas, R. de C. G. Influence of a high-fat diet on gut microbiota, intestinal permeability and metabolic endotoxaemia. Br. J. Nutr. 108, 801-809 (2012).

6. Guillemot-Legris, O. \& Muccioli, G. G. Obesity-Induced Neuroinflammation: Beyond the Hypothalamus. Trends Neurosci. 40, 237-253 (2017).

7. Glass, C. K., Saijo, K., Winner, B., Marchetto, M. C. \& Gage, F. H. Mechanisms Underlying Inflammation in Neurodegeneration. Cell 140, 918-934 (2010).

8. Zhang, R. et al. Circulating endotoxin and systemic immune activation in sporadic Amyotrophic Lateral Sclerosis (sALS). J. Neuroimmunol. 206, 121-124 (2009).

9. Leicht, H. et al. Predictors of costs in dementia in a longitudinal perspective. PloS One 8, e70018 (2013).

10. Madabhushi, R., Pan, L. \& Tsai, L.-H. DNA damage and its links to neurodegeneration. Neuron 83, 266-282 (2014).

11. Deczkowska, A. et al. Disease-Associated Microglia: A Universal Immune Sensor of Neurodegeneration. Cell 173, 1073-1081 (2018).

12. Mueller, S. G. et al. Hippocampal Atrophy Patterns in Mild Cognitive Impairment and Alzheimer's Disease. Hum. Brain Mapp. 31, 1339-1347 (2010). 
13. Schröder, J. \& Pantel, J. Neuroimaging of hippocampal atrophy in early recognition of Alzheimer's disease--a critical appraisal after two decades of research. Psychiatry Res. Neuroimaging 247, 71-78 (2016).

14. Avila, J., Gómez de Barreda, E., Engel, T., Lucas, J. J. \& Hernández, F. Tau phosphorylation in hippocampus results in toxic gain-of-function. Biochem. Soc. Trans. 38, 977-980 (2010).

15. Sebastián-Serrano, Á., de Diego-García, L. \& Díaz-Hernández, M. The Neurotoxic Role of Extracellular Tau Protein. Int. J. Mol. Sci. 19, (2018).

16. Del Olmo, N. \& Ruiz-Gayo, M. Influence of High-Fat Diets Consumed During the Juvenile Period on Hippocampal Morphology and Function. Front. Cell. Neurosci. 12, (2018).

17. Polyzos, S. A., Kountouras, J. \& Mantzoros, C. S. Obesity and nonalcoholic fatty liver disease: From pathophysiology to therapeutics. Metabolism (2018).

doi:10.1016/j.metabol.2018.11.014

18. Takahashi, Y., Sugimoto, K., Inui, H. \& Fukusato, T. Current pharmacological therapies for nonalcoholic fatty liver disease/nonalcoholic steatohepatitis. World J. Gastroenterol. WJG 21, 3777-3785 (2015).

19. Eshraghian, A. Current and emerging pharmacological therapy for non-alcoholic fatty liver disease. World J. Gastroenterol. 23, 7495-7504 (2017).

20. Moelands, S. V., Lucassen, P. L., Akkermans, R. P., De Grauw, W. J. \& Van de Laar, F. A. Alpha-glucosidase inhibitors for prevention or delay of type 2 diabetes mellitus and its associated complications in people at increased risk of developing type 2 diabetes mellitus. Cochrane Database Syst. Rev. 12, CD005061 (2018).

21. Honda, Y. et al. Pemafibrate, a novel selective peroxisome proliferator-activated receptor alpha modulator, improves the pathogenesis in a rodent model of nonalcoholic steatohepatitis. Sci. Rep. 7, 42477 (2017).

22. Deardorff, W. J. \& Grossberg, G. T. Targeting neuroinflammation in Alzheimer's disease: evidence for NSAIDs and novel therapeutics. Expert Rev. Neurother. 17, 17-32 (2017).

23. Dou, K.-X. et al. Comparative safety and effectiveness of cholinesterase inhibitors and memantine for Alzheimer's disease: a network meta-analysis of 41 randomized controlled trials. Alzheimers Res. Ther. 10, 126 (2018). 
24. Cicero, A. F. G., Colletti, A. \& Bellentani, S. Nutraceutical Approach to Non-Alcoholic Fatty Liver Disease (NAFLD): The Available Clinical Evidence. Nutrients 10, (2018).

25. Mollazadeh, H., Mahdian, D. \& Hosseinzadeh, H. Medicinal plants in treatment of hypertriglyceridemia: A review based on their mechanisms and effectiveness.

Phytomedicine Int. J. Phytother. Phytopharm. 53, 43-52 (2019).

26. Amiot, M. J., Riva, C. \& Vinet, A. Effects of dietary polyphenols on metabolic syndrome features in humans: a systematic review. Obes. Rev. Off. J. Int. Assoc. Study Obes. 17, 573586 (2016).

27. Amor, S., Châlons, P., Aires, V. \& Delmas, D. Polyphenol Extracts from Red Wine and Grapevine: Potential Effects on Cancers. Dis. Basel Switz. 6, (2018).

28. Zhang, Y. et al. Chemical compositional, biological, and safety studies of a novel maple syrup derived extract for nutraceutical applications. J. Agric. Food Chem. 62, 6687-6698 (2014).

29. Maisuria, V. B., Hosseinidoust, Z. \& Tufenkji, N. Polyphenolic extract from maple syrup potentiates antibiotic susceptibility and reduces biofilm formation of pathogenic bacteria. Appl. Environ. Microbiol. 81, 3782-3792 (2015).

30. Liu, W. et al. Anti-glycation and anti-oxidative effects of a phenolic-enriched maple syrup extract and its protective effects on normal human colon cells. Food Funct. 8, 757-766 (2017).

31. Ma, H. et al. Effects of a Standardized Phenolic-Enriched Maple Syrup Extract on $\beta$-Amyloid Aggregation, Neuroinflammation in Microglial and Neuronal Cells, and $\beta$-Amyloid Induced Neurotoxicity in Caenorhabditis elegans. Neurochem. Res. 41, 2836-2847 (2016).

32. More, V. R. et al. Keap1 knockdown increases markers of metabolic syndrome after longterm high fat diet feeding. Free Radic. Biol. Med. 61, 85-94 (2013).

33. Domínguez-Pantoja, M. et al. CD38 protein deficiency induces autoimmune characteristics and its activation enhances IL-10 production by regulatory B cells. Scand. J. Immunol. 87, e12664 (2018).

34. Simons, K. H. et al. The role of CD27-CD70-mediated T cell co-stimulation in vasculogenesis, arteriogenesis and angiogenesis. Int. J. Cardiol. 260, 184-190 (2018). 
35. Grosjean, M. B. et al. Immunohistochemical characterization of Fas (CD95) and Fas Ligand (FasL/CD95L) expression in the injured brain: relationship with neuronal cell death and inflammatory mediators. Histol. Histopathol. 22, 235-250 (2007).

36. Anhê, F. F. et al. Gut Microbiota Dysbiosis in Obesity-Linked Metabolic Diseases and Prebiotic Potential of Polyphenol-Rich Extracts. Curr. Obes. Rep. 4, 389-400 (2015).

37. Darnaud, M., Faivre, J. \& Moniaux, N. Targeting gut flora to prevent progression of hepatocellular carcinoma. J. Hepatol. 58, 385-387 (2013).

38. Fröhlich, E. E. et al. Cognitive impairment by antibiotic-induced gut dysbiosis: Analysis of gut microbiota-brain communication. Brain. Behav. Immun. 56, 140-155 (2016).

39. Dudvarski Stankovic, N., Teodorczyk, M., Ploen, R., Zipp, F. \& Schmidt, M. H. H. Microgliablood vessel interactions: a double-edged sword in brain pathologies. Acta Neuropathol. (Berl.) 131, 347-363 (2016).

40. Zlokovic, B. V. The blood-brain barrier in health and chronic neurodegenerative disorders. Neuron 57, 178-201 (2008).

41. Brochard, V. et al. Infiltration of CD4+ lymphocytes into the brain contributes to neurodegeneration in a mouse model of Parkinson disease. J. Clin. Invest. 119, 182-192 (2009).

42. Koga, S. et al. Effects of diet-induced obesity and voluntary exercise in a tauopathy mouse model: Implications of persistent hyperleptinemia and enhanced astrocytic leptin receptor expression. Neurobiol. Dis. 71, 180-192 (2014).

43. Lucas, S. et al. Interleukin-7 Regulates Adipose Tissue Mass and Insulin Sensitivity in HighFat Diet-Fed Mice through Lymphocyte-Dependent and Independent Mechanisms. PLOS ONE 7, e40351 (2012).

44. Araujo, D. M. \& Cotman, C. W. Trophic effects of interleukin-4, -7 and -8 on hippocampal neuronal cultures: potential involvement of glial-derived factors. Brain Res. 600, 49-55 (1993).

45. Paul, S. R. \& Schendel, P. The cloning and biological characterization of recombinant human interleukin 11. Int. J. Cell Cloning 10, 135-143 (1992). 
46. Chen, W.-W., Zhang, X. \& Huang, W.-J. Role of neuroinflammation in neurodegenerative diseases (Review). Mol. Med. Rep. 13, 3391-3396 (2016).

47. Cai, D. Neuroinflammation and neurodegeneration in overnutrition-induced diseases. Trends Endocrinol. Metab. TEM 24, 40-47 (2013).

48. Nuzzo, D. et al. Insulin Resistance as Common Molecular Denominator Linking Obesity to Alzheimer's Disease. Curr. Alzheimer Res. 12, 723-735 (2015).

49. Pei, Z. et al. MAC1 mediates LPS-induced production of superoxide by microglia: the role of pattern recognition receptors in dopaminergic neurotoxicity. Glia 55, 1362-1373 (2007).

50. Bodea, L.-G. et al. Neurodegeneration by Activation of the Microglial ComplementPhagosome Pathway. J. Neurosci. 34, 8546-8556 (2014).

51. Singh, J. et al. The role of polar interactions in the molecular recognition of CD4OL with its receptor CD40. Protein Sci. Publ. Protein Soc. 7, 1124-1135 (1998).

52. Amitani, M., Asakawa, A., Amitani, H. \& Inui, A. The role of leptin in the control of insulinglucose axis. Front. Neurosci. 7, (2013).

53. Morris, D. L. \& Rui, L. Recent advances in understanding leptin signaling and leptin resistance. Am. J. Physiol. Endocrinol. Metab. 297, E1247-1259 (2009).

54. Scott, M. M. et al. Leptin Targets in the Mouse Brain. J. Comp. Neurol. 514, 518-532 (2009).

55. Bjørbæk, C. Central Leptin Receptor Action and Resistance in Obesity. J. Investig. Med. Off. Publ. Am. Fed. Clin. Res. 57, 789-794 (2009).

56. McGregor, G. \& Harvey, J. Food for thought: Leptin regulation of hippocampal function and its role in Alzheimer's disease. Neuropharmacology 136, 298-306 (2018).

57. Bonda, D. J. et al. Dysregulation of leptin signaling in Alzheimer disease: evidence for neuronal leptin resistance. J. Neurochem. 128, 162-172 (2014). 
58. Sobesky, J. L. et al. High-fat diet consumption disrupts memory and primes elevations in hippocampal IL-1 $\beta$, an effect that can be prevented with dietary reversal or IL-1 receptor antagonism. Brain. Behav. Immun. 42, 22-32 (2014).

59. Akdis, M. et al. Interleukins (from IL-1 to IL-38), interferons, transforming growth factor $\beta$, and TNF- $\alpha$ : Receptors, functions, and roles in diseases. J. Allergy Clin. Immunol. 138, 9841010 (2016).

60. González, H. \& Pacheco, R. T-cell-mediated regulation of neuroinflammation involved in neurodegenerative diseases. J. Neuroinflammation 11, 201 (2014).

61. Coles, A. H. et al. A High-Throughput Method for Direct Detection of Therapeutic Oligonucleotide-Induced Gene Silencing In Vivo. Nucleic Acid Ther. 26, 86-92 (2016).

62. Chen, L. et al. TLR4 inhibitor attenuates amyloid- $\beta$-induced angiogenic and inflammatory factors in ARPE-19 cells: Implications for age-related macular degeneration. Mol. Med. Rep. 13, 3249-3256 (2016).

63. Tai, L. M. et al. APOE-modulated A $\beta$-induced neuroinflammation in Alzheimer's disease: current landscape, novel data, and future perspective. J. Neurochem. 133, 465-488 (2015).

64. Miyoshi, J. et al. Minimizing confounders and increasing data quality in murine models for studies of the gut microbiome. PeerJ 6, e5166 (2018).

65. Saarinen, N. M. \& Thompson, L. U. Prolonged administration of secoisolariciresinol diglycoside increases lignan excretion and alters lignan tissue distribution in adult male and female rats. Br. J. Nutr. 104, 833-841 (2010).

66. Roncaglia, L., Amaretti, A., Raimondi, S., Leonardi, A. \& Rossi, M. Role of bifidobacteria in the activation of the lignan secoisolariciresinol diglucoside. Appl. Microbiol. Biotechnol. 92, 159-168 (2011).

67. Quartieri, A. et al. Detection of novel metabolites of flaxseed lignans in vitro and in vivo. Mol. Nutr. Food Res. 60, 1590-1601 (2016).

68. Kore, R. A. \& Abraham, E. C. Inflammatory cytokines, interleukin-1 beta and tumor necrosis factor-alpha, upregulated in glioblastoma multiforme, raise the levels of CRYAB in exosomes secreted by U373 glioma cells. Biochem. Biophys. Res. Commun. 453, 326-331 (2014). 
69. Baranello, R. J. et al. Amyloid-beta protein clearance and degradation ( $A B C D)$ pathways and their role in Alzheimer's disease. Curr. Alzheimer Res. 12, 32-46 (2015).

70. Varga, T., Czimmerer, Z. \& Nagy, L. PPARs are a unique set of fatty acid regulated transcription factors controlling both lipid metabolism and inflammation. Biochim. Biophys. Acta 1812, 1007-1022 (2011).

71. Džoljić, E., Grabatinić, I. \& Kostić, V. Why is nitric oxide important for our brain? Funct. Neurol. 30, 159-163 (2015).

72. Bunton-Stasyshyn, R. K. A., Saccon, R. A., Fratta, P. \& Fisher, E. M. C. SOD1 Function and Its Implications for Amyotrophic Lateral Sclerosis Pathology: New and Renascent Themes. Neurosci. Rev. J. Bringing Neurobiol. Neurol. Psychiatry 21, 519-529 (2015).

73. Tarantini, S. et al. Nrf2 Deficiency Exacerbates Obesity-Induced Oxidative Stress, Neurovascular Dysfunction, Blood-Brain Barrier Disruption, Neuroinflammation, Amyloidogenic Gene Expression, and Cognitive Decline in Mice, Mimicking the Aging Phenotype. J. Gerontol. Ser. A 73, 853-863 (2018).

74. Feng, J. et al. Induction of CD36 expression by oxidized LDL and IL-4 by a common signaling pathway dependent on protein kinase C and PPAR-gamma. J. Lipid Res. 41, 688-696 (2000). 


\section{MANUSCRIPT 3}

\section{In preparation for submission to Molecular Metabolism}

Evaluation of a food grade maple syrup extract against western diet-induced obesity in male C57BL/6 mice.

Nicholas A. DaSilva ${ }^{1}$, Marisa Pfohl ${ }^{1}$, Emily Marques ${ }^{1}$, Benjamin Barlock ${ }^{1}$, Hang Ma $^{1}$, Wei Wei ${ }^{1}$, Chang Liu ${ }^{1}$, Juliana Agudelo ${ }^{1}$, Shelby L. Johnson², Kenneth N. Rose ${ }^{2}$, Michael Goedken ${ }^{3}$, Fatemeh Akhlaghi ${ }^{1}$, Angela L. Slitt ${ }^{1,2}$, Navindra P. Seeram ${ }^{1,2}$

Affiliations:

${ }^{1}$ Department of Biomedical and Pharmaceutical Sciences, University of Rhode Island, Kingston, RI USA

${ }^{2}$ George and Anne Ryan Institute for Neuroscience, University of Rhode Island, Kingston, RI USA

${ }^{3}$ Rutgers Translational Sciences, Rutgers University, Piscataway, NJ, USA

Corresponding Authors:

Navindra Seeram, Ph.D.; University of Rhode Island, 495B, Avedisian Hall, 7 Greenhouse Rd.

Kingston, RI, 02881, USA, Phone: 401-874-9367, Email: nseeram@uri.edu

Angela L. Slitt, Ph.D.; University of Rhode Island, 395D, Avedisian Hall, 7 Greenhouse Rd.

Kingston, RI 02881, USA, Phone: 401-874-5020, Email: aslitt@uri.edu 


\section{Abstract}

Background: Obesity is a diet-induced disease that stems into numerous pathologies such as metabolic syndrome, type II diabetes mellitus, Nonalcoholic Steatohepatitis, Fatty Liver disease and even cancer. Chronic low grade inflammation is linked in the etiology of these diseases. Polyphenol rich nutraceuticals such as Maple Syrup Extract (MSX) may prevent or reduce the severity of these inflammatory processes.

Methods: MSX, was evaluated in a murine diet-induced obesity model for a period of 18 weeks. Body weight and food consumption were monitored in addition to measuring Fasting Blood Glucose, Glucose, and Insulin Tolerance during this study. IL-6 was quantified from serum following necropsy. Liver and White Adipose Tissue were excised and weighed at the time of necropsy. Livers were sectioned, stained using $\mathrm{H} \& \mathrm{E}$ and scored for histological features associated with NASH/NAFLD. Livers were also subjected to bottom-up proteomic analysis for significant protein changes.

Results: 18 weeks of high fat high fructose consumption paired with sedentary behavior caused significant increases in body weight, serum IL-6 concentrations, glucose and insulin intolerance as compared to low fat diet fed mice. The addition of MSX $0.5 \% \mathrm{w} / \mathrm{w}$ exacerbated liver steatosis whereas MSX $0.05 \% \mathrm{w} / \mathrm{w}$ failed to improve histological scoring as compared to high fat diet control mice.

Conclusion: MSX supplementation in high fat diet feed did not ameliorate the progression of fatty liver disease in this rodent model as compared to our previous pilot study. The addition of MSX at $0.05 \%$ did significantly reduce pro-inflammatory serum IL- 6 concentrations as compared to high fat diet control mice. The addition of fructose and glucose in drinking water combined with the duration of diet exposure may have masked the beneficial effects of MSX witnessed in previous studies. 


\section{Background}

Obesity, which affects millions of individuals on a global scale, is linked to a number of secondary life-threatening diseases. These diseases such as type II diabetes mellitus (T2DM), Non-alcoholic Steatohepatitis (NASH), Non-alcoholic Fatty Liver Disease(NAFLD), Cardiovascular Disease $(C D)$, Kidney Disease (KD) and even cancer ${ }^{1-3}$. These malignances, combined with glucose intolerance and insulin resistance make of the spectrum of diseases in metabolic syndrome (MetS) ${ }^{4,5}$. These MetS diseases have become more prevalent in the United States and contribute to rising healthcare costs. Diet-induced obesity, or the life-long ingestion of saturated fats and sugars (fructose and sucrose) may be a causal link to these diseases partially through the dysbiosis of microbes within our gut ${ }^{4,6}$. By favoring the growth of pathogenic bacteria and depleting beneficial populations of bacteria, the luminal concentration of harmful metabolites such as lipopolysaccharide (LPS) in the gut increase over time ${ }^{7}$.

These metabolites permeate through weakened intestinal barriers into the hepatic portal system to ultimately effect the Liver and other organ systems (termed endotoxemia) ${ }^{4,7}$. These metabolites, namely LPS, initiate an inflammatory response in circulating monocytes, resident macrophages, and immune cells (i.e. B-cells, T-cells) ${ }^{8-10}$. The chronic activation and subsequent release of pro-inflammatory mediators leads to cell damage and death through disrupting mitochondrial function, caspase activation, and in some cases oncogene activation inciting cancer growth ${ }^{11,12}$. The immune system, therefore, is seen as a potential therapeutic target in the pathogenesis of these metabolic diseases ${ }^{13,14}$. 
While there is no specific treatment for MetS, prophylactic measures namely diet change are seen as the most cost effective and impactful ${ }^{15,16}$. Incorporation of natural products and extracts into a diet have been heavily researched and touted as having fewer side effects and lower cost as compared to synthetic products ${ }^{17-21}$. One under-researched source of these immunomodulatory natural products is maple syrup. Derived from the boiling of sap from sugar maple species (Acer saccharum L), maple syrup and its derived products (maple sugar, maple water etc.) contain over 60 unique phytochemicals ${ }^{22,23}$. Of these 60 constituents, polyphenols are the most abundant along with sugars, vitamins, and minerals ${ }^{23}$.

Our group in particular has developed a novel food gradem phenolic-enriched maple syrup extract (MSX) derived from the darker, off-flavor, fractions of maple syrup ${ }^{24}$. MSX, which contains a similar phytochemical profile to maple syrup, contains a number of polyphenols from various chemical classes including phenolic acids, lignans, stilbenes and coumarins ${ }^{24}$. Previous studies from our group demonstrated the anti-inflammatory and anti-glycemic activity of MSX in murine and human macrophages, microglia, neurons, and hepatocytes ${ }^{24,25}$. MSX has also been found to be safe in rats at doses up to $1000 \mathrm{mg} / \mathrm{kg} / \mathrm{day}^{24}$.

In a 12 week pilot study, male C57BL/6 mice were fed a diet rich in fats to promote obesity. MSX supplementation in high fat diet (HFD) fed mice lowered body weight, white adipose tissue weight, and liver weight as compared to their HFD controls. Moreover, lipid metabolism, fatty acid oxidation, and inflammatory genes were also modulated in livers and hippocampi of these MSX supplemented mice. 
Previous findings prompted further investigation into the probable mechanisms associated with MSX consumption. In the present study, male C57BL/6 mice were fed either a standard lab chow or an American lifestyle induced obesity syndrome (ALIOS) diet to promote inflammation, glucose intolerance and hepatic fatty acid accumulation. These diets were formulated with or without MSX for 18 weeks with the ultimate goal of modulating the progression of MetS.

\section{Materials and Methods}

\section{Animal Treatment and Study Design}

C57BL/6 $(n=40)$ aged 4 weeks were purchased from Jackson Laboratories (Bar Harbor , ME USA) and housed with at least two mice per cage under 12 hour light/day cycles. Mice we allowed to acclimate for 1 week before having bedding pooled and redistributed weekly for two additional weeks. Mice are fed either a Low Fat Diet (LFD)(12\% kcal from fat)( $n=8)$, LFD with 0.5\% w/w MSX (LMSX0.5)(n=6) , LFD with 0.05\% w/w MSX (LMSX0.05)(n=6), High Fat Diet

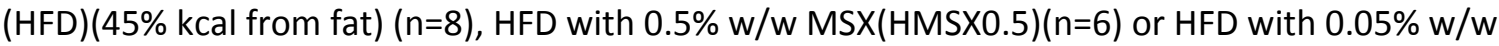
MSX (HMSX0.05)(n=6). Bedding is pooled weekly within treatment groups, mixed and

redistributed within the treatment group to account for a microbial cohousing effect ${ }^{20,21,26}$. Food intake, body weights and water consumption are monitored weekly. At the end of 18 weeks, 40 mice were sacrificed and whole blood collected using cardiac puncture. Whole blood was centrifuged and immediately separated into two aliquots for future analysis. Mice were sacrificed by isoflurane and decapitation following blood collection. 
This study and its protocols were approved by and conducted in accordance with the University of Rhode Island IACUC Institutional Animal Use Committee (Approval \# AN09-07-004).

\section{Diet Selection}

The ALIOS diet or the High Fat Diet component, models a western diet paired with sedentary behavior (placing food directly inside the cage) and sugar rich drinking water. The diet consists of hydrogenated vegetable oil (saturated fats) and cholesterol $(0.2 \% \mathrm{w} / \mathrm{w})$ in addition to a high sugar water component (42 g/L: 55\% w/v Fructose, 45\% w/v Glucose)(TD.120330). Diets were prepared by Envigo-Teklad (Madison, WI USA) and chosen based on previously cited studies that show the development of NASH phenotypes including liver lipid accumulation and fibrosis within 16 weeks. The dietary inclusion of cholesterol and high fructose/glucose in

drinking water also has been shown to further promote the NASH phenotype. ${ }^{9,27,28}$ A custom grain free, phytoestrogen free diet (TD.2020) was used as a Low Fat Diet control.

\section{Glucose Tolerance Test (GTT) and Insulin Tolerance Test (ITT)}

Mice were fasted for 6 hours in both GTT and ITT procedures. Glucose tolerance was measured by sampling blood glucose with Bayer Contour Next Glucometers (Ascensia Diabetes, Parsippany NJ, USA) after an intraperitoneal (i.p.) bolus of glucose $(10 \mathrm{ml} / \mathrm{kg})($ Sigma Aldrich, St. Louis, MO USA). Insulin Tolerance was also measured in a similar fashion however measuring blood glucose after a single dose $(0.75 \mathrm{U} / \mathrm{kg}$ ) of human insulin (Sigma Aldrich, St. Louis, MO USA) was administered via i.p. injection. Blood glucose was recorded at the following timepoints: 0 , $15,30,45,60,90,120$, and 150 minutes following i.p. injection. Average area under the curve was then calculated prior to statistical analysis. 


\section{Histology and Pathology}

Livers were stored in Neutral Buffered Formalin (Sigma Aldrich, St. Louis MO) for at least 24 hours prior to sectioning and staining. Sections of liver were stained with Hemotoxylin and Eosin (H\&E) and scored by a board certified veterinary pathologist using the method described by Kleiner et $\mathrm{al}^{29}$. Semi-quantitative scores were assigned based on the following histopathological features: Lipid Accumulation, Necrosis, Inflammation, Biliary Hyperplasia from 0 (0\% injury/death) , 1 (<10\% injury/death), 2 (10-25 \% injury/death), 3 ( $25-40 \%$ injury/death) , 4 (40-50\% injury/death) , or 5 ( >50\% injury/death) and Tumor/Lesion incidence ( 0 absent or 1 present). Scores were then rank ordered before determining statistical significance. Scores from Inflammation, Lipid Accumulation, and Necrosis were combined and categorized to establish a NAFLD activity score (NAS) based on van der Heijden et al where samples scored 0-3 represent non-steatohepatitis, 4 borderline steatohepatitis, and scores $\geq 5$ steatohepatitis ${ }^{30}$

\section{Cell Culture of Human Monocytes and Macrophages}

Healthy human donor monocytes (Donor 338) were obtained from Astarte Biologics (Bothell, WA USA) and seeded directly into 24 wells plates as stated by vendor protocols. Monocytes were then treated with MSX at a dose of $10 \mu \mathrm{g} / \mathrm{mL}$ for 1 hour prior to being exposed to Lipopolysaccharide (LPS) (Sigma Aldrich, St. Louis, MO USA) for 23 hours. Resveratrol at 20 $\mu \mathrm{M}$ was also used for comparison and as a positive control (Sigma Aldrich, St. Louis, MO). DMSO at $0.1 \% \mathrm{v} / \mathrm{v}$ was used as a vehicle control (Sigma Aldrich, St. Louis, MO USA). Following incubation with LPS for 23 hours, media was centrifuged before being stored for future analysis. Secreted TNF $\alpha$ and IL-6 were quantified in media using Biolegend's Human Deluxe ELISA (San Diego, CA USA). 
THP-1 Human Monocytes were donated by Dr. Nisanne Ghonem of the University of Rhode Island's College of Pharmacy and were maintained using the ATCC's methods of culturing. Cells were maintained in RPMI 1640 Media supplemented with 10\% v/v Fetal Bovine Serum (Gibco, Grand Island, NY USA), 0.5\% Penicillin/Streptomycin Antibiotics and $50 \mu \mathrm{M}$ of $\beta$ Mercaptoethanol (Biologic Grade, Sigma Aldrich, St. Louis , MO USA).

Findings with healthy human donor monocytes were further investigated using phorbol 12-myristate 13-acetate (PMA) (Sigma Aldrich, St. Louis, MO USA) differentiation which were carried out according to previously described and optimized methodologies ${ }^{31}$. In brief, THP-1 cells were seeded into 24 well plates at a density of approximately 200,000 cells $/ \mathrm{mL}$ and allowed to incubate for at least 24 hours prior to PMA exposure. PMA was then added directly to each well at final concentration of $25 \mathrm{nM}$ for 48 hours. Following this period, cells were checked for adherence and proper morphology before exchanging existing differentiation media to serum free media for at least 24 hours prior to compound treatment.

As part of a preliminary measure, the effects of MSX on IL- 6 and TNF $\alpha$ secretion at 6 and 24 hours were assayed. MSX at concentrations of 50 and $100 \mu \mathrm{g} / \mathrm{mL}$ were added to each respective set of wells $(n=4)$ for 1 hour before being exposed to LPS at $1 \mu \mathrm{g} / \mathrm{mL}$ for 6 hours. Following LPS exposure, media was collected, centrifuged and assayed for IL-6 and TNF $\alpha$. 


\section{THP-1 Macrophage Cytokine Multiplex}

After determining the optimal timeframe for measuring cytokine release in our THP-1 macrophage system, the experiments were repeated again in 24 well plates but assaying Vehicle Control (DMSO), LPS alone $(1 \mu \mathrm{g} / \mathrm{mL})$, Resveratrol at 10 and $20 \mu \mathrm{M}$, and MSX at $0.1,1,10$ and $100 \mu \mathrm{g} / \mathrm{mL}$. Each was pre-treated for 1 hour with the indicated compound or extract before being replaced with media containing $1 \mu \mathrm{g} / \mathrm{mL}$ of LPS for 6 hours. After which, media was collected, centrifuged, and frozen in aliquots for cytokine analysis. Once again, IL-6 and TNF $\alpha$ were measured along with IL-1 $\beta$, IL-RA, IL-10, and MCP-1 using a Luminex XMAP based, Procarta Plex Multiplex (Invitrogen, Carlsbad, CA) . Observed concentrations were calculated using the software available on the Biorad Bioplex 200.

\section{Chemicals and Reagents}

TPCK-treated trypsin, trypsin digested $\beta$-galactosidase and mass spectrometer tuning solution were purchased from SCIEX, Framingham, MA. Acquity UPLC Peptide BEH C18 analytical column and VanGuard pre-columns were procured from Waters Corp. (Waltham, MA). 1,4Dithiothreitol (DTT) was obtained from Roche Diagnostics (Indianapolis, IN). Bovine serum albumin, sodium deoxycholate and iodoacetamide (IAA) were procured from Sigma Aldrich (St. Louis, MO). MS grade acetonitrile and formic acid were purchased from ThermoFisher, Waltham, MA. 


\section{Mouse liver homogenate preparation}

Approximately $100 \mathrm{mg}$ of mouse liver tissue was cut, weighed and homogenized in 1000 $\mu \mathrm{l}$ of homogenization buffer ( $8 \mathrm{M}$ urea, $50 \mathrm{mM}$ Triethylammonium bicarbonate, $5 \mathrm{mM}$ DTT v/v) using a bead homogenizer (Omni Bead Ruptor, Kennesaw GA) ${ }^{32}$. Supernatant was collected after spinning at $1,000 \mathrm{~g}$ for $5 \mathrm{~min}$. Pierce BCA protein assay kit (ThermoFisher Scientific, Waltham, MA) was used to determine total protein concentration. Samples were diluted to a stock concentration of $5 \mathrm{mg} / \mathrm{ml}$.

\section{In-solution trypsin digestion}

Protein digestion was conducted as described previously with few adaptations ${ }^{32,33}$. Samples (500 $\mu$ g protein), along with an internal control of $2 \mu \mathrm{g}$ bovine serum albumin (BSA), were denatured with $25 \mu \mathrm{L} \mathrm{DTT}(100 \mathrm{mM})$ at $35^{\circ} \mathrm{C}$ for $30 \mathrm{~min}$ in a shaking water bath (100 rpm). Samples were then alkylated in the dark with $25 \mu \mathrm{L}$ IAA $(200 \mathrm{mM})$ at room temperature for 30 min. After alkylation, samples were concentrated using the cold water, methanol and chloroform (1:2:1) precipitation method followed by centrifugation at $10000 \mathrm{rpm}, 5 \mathrm{~min}$ at $10^{\circ} \mathrm{C}$. Ice-cold methanol was used to wash the protein pellet. The pellet was then suspended in $200 \mu \mathrm{L}$ of $50 \mathrm{mM}$ ammonium bicarbonate $\left(\mathrm{pH}^{\sim} 8\right)$ containing $3 \% \mathrm{w} / \mathrm{v}$ sodium deoxycholate (DOC). Further, $125 \mu \mathrm{L}$ of the sample was then spiked with TPCK-treated trypsin $(12.5 \mu \mathrm{g})$ and samples were transferred into digestion tubes (PCT MicroTubes, Pressure Biosciences Inc., Easton, MA). The barocycler was run at $35^{\circ} \mathrm{C}$, for 75 cycles with 60 -sec pressure-cycle (50-sec high pressure, 10-sec ambient pressure, $25 \mathrm{kpsi})$. Following the first run, $12.5 \mu \mathrm{g}$ trypsin was added to each sample and the barocycler was run again at the above settings. 
After the barocycler, $12.5 \mu \mathrm{L}$ of acetonitrile (1:1, v/v containing $5 \%$ formic acid) was added to $125 \mu \mathrm{L}$ of digested peptides sample in order to precipitate DOC. Supernatant was collected after samples were spun at $10,000 \mathrm{rpm}$ for $5 \mathrm{~min}$ at $10^{\circ} \mathrm{C}$. Subsequently, $25 \mu \mathrm{L}$ of the digested peptide sample was injected and analyzed using LC-MS/MS.

\section{LC-MS/MS Analysis}

Data-dependent analysis (DDA) was performed in positive ionization mode using a DuoSpray $^{\mathrm{TM}}$ ion source on a Sciex 5600 TripleTOF $^{\mathrm{TM}}$ mass spectrometer (AB Sciex, Concord, Canada). Separation was achieved using an Acquity UPLC HClass system (Waters Corp., Milford, MA, USA). Ion spray voltage floating (ISVF) was kept at $5500 \mathrm{~V}$ while the source temperature (TEM) was $500^{\circ} \mathrm{C}$. Gas 1 (GS1), gas 2 (GS2) and curtain gas (CUR) were set to 55,60 and 25 psi, respectively. Declustering potential (DP), collision energy (CE) and collision energy spread (CES) were set at 120, 10 and 5 respectively. During the survey scan, all the ions with a charge state of 2 to 4 , mass range of $m / z 300-1250$ and exceeding $25 \mathrm{cps}$ were used for MS/MS analysis. Former target ions were excluded for $8 \mathrm{sec}$ and the mass tolerance for TOF-MS was $50 \mathrm{mDa}$ with a 100 milliseconds accumulation time. For the product scan, data were acquired from 100 to $1250 \mathrm{~m} / \mathrm{z}$ with an accumulation time of 75 milliseconds with a total cycle time of $3.5 \mathrm{sec}$. Product ion analysis was done under dynamic accumulation and rolling collision energy dependent on the $m / z$ of the ion. All the parameters for SWATH acquisition were similar as described above except the following: Source temperature (TEM) was $400^{\circ} \mathrm{C}$, GS 1 was 55 psi, and TOF masses were collected from m/z 300 to 1500. The total cycle time for SWATH acquisition was $3.95 \mathrm{sec}$. SWATH data was acquired (m/z 400-1100) over 70 SWATH windows per cycles with a window size of $\mathrm{m} / \mathrm{z} 10$. 
Chromatographic separation was achieved over 90 min gradient method at $100 \mu \mathrm{L} / \mathrm{min}$ on an Acquity UPLC Peptide BEH C18 $(2.1$ X 150 mm, $300 \AA, 1.7 \mu \mathrm{m})$ preceded by an Acquity VanGuard pre-column (2.1 X $5 \mathrm{~mm}, 300 \AA \AA, 1.7 \mu \mathrm{m})$. Mobile phase A was $100 \%$ water, $0.1 \%$ formic acid and mobile phase B was $100 \%$ acetonitrile, $0.1 \%$ formic acid). Gradient conditions used were $98 \%$ A from 0 to $5 \mathrm{~min}, 98 \%$ to $70 \%$ A from 5 to $65 \mathrm{~min}, 70 \%$ to $50 \%$ A from 65 to $70 \mathrm{~min}, 50 \%$ to $20 \%$ A from 70 to $75 \mathrm{~min}, 20 \%$ A was held from 75 to $80 \mathrm{~min}$, and $20 \%$ A to $98 \%$ A from 80 to $85 \mathrm{~min}$.

The gradient was held at initial conditions from $85 \mathrm{~min}$ until the end of the run to equilibrate the column before the start of next run. The flow was diverted to waste for the first 5 minutes and last 10 minutes of the acquisition. Autosampler was maintained at $10^{\circ} \mathrm{C}$, and the column was kept at $50^{\circ} \mathrm{C}$. Trypsin-digested $\beta$-galactosidase peptides were injected to monitor TOF detector mass calibration every four samples.

\section{Data processing}

The absolute level of proteins was determined from DDA data using "Total Protein Approach" ${ }^{34}$. Mouse liver homogenate samples were analyzed as previously described using MaxQuant (ver 1.5.2.10) ${ }^{32,34}$. The proteins were searched on Andromeda search engine against the Swiss-Prot human protein database (updated Apr 2018) at $1 \%$ false discovery rate (FDR) ${ }^{35}$. Cysteine carbamidomethylation was selected as a fixed modification, and oxidation (M) and acetyl ( $\mathrm{N}$-term) were used as variable modifications. Label-free quantification was performed with a ratio count of 1 , and a maximum of two missed cleavages was allowed. All the other MaxQuant settings were kept as default values. The absolute protein levels were calculated using "Total Protein Approach" from LFQ intensities ${ }^{34}$. 
The protein concentrations were expressed as picomoles of protein per gram liver tissue (pmol/g liver).

Normalized Protein conc. $(\mathrm{p})=$ Protein conc $(\mathrm{p}) \times$ PPGL [pmol/g liver]

where PPGL denotes the yield of milligrams of total protein per gram of liver tissue.

\section{Statistical Analysis}

All statistical calculations were performed using Prism version 8.0 (Graphpad, La Jolla CA USA). Data is shown as mean \pm SEM. Unless otherwise indicated, One-way analysis of Variance (ANOVA) with Dunnett's corrections for multiple comparisons were used to describe statistical significance. P values herein are designated as follows: $p<0.05^{*}, p<0.01 * *, p<0.001 * * *, p<$ $0.0001 * * * *$.

\section{Results}

\section{MSX in LFD and HFD lowers serum IL-6 concentration in HFD mice and mitgates liver and} white adipose tissue weight increases

During the course of this study, approximately half of the mice were allowed to consume a low fat diet (LFD) with or without MSX at either $0.5 \%$ or $0.05 \%$ included in the feed. The remaining half were fed a high fat diet composed of $22 \%$ hydrogenated vegetable oil , $0.2 \%$ cholesterol, and fructose-glucose water (HFD) also with or without MSX at either $0.5 \%$ or $0.05 \%$ $\mathrm{w} / \mathrm{w}$ included in the diet. At week $18 \mathrm{HFD}$ mice weighed approximately $42.28 \mathrm{~g} \pm 0.81 \mathrm{~g}$ as compared to the LFD group which are approximately $35.55 \mathrm{~g} \pm 0.79 \mathrm{~g}$ (Figure $3 \mathrm{~A}$ ). The addition of MSX at $0.05 \% \mathrm{w} / \mathrm{w}$ or $0.5 \% \mathrm{w} / \mathrm{w}$ did not significantly decrease body weight at week 18 . 
At the time of sacrifice, liver, kidneys, adipose tissue (mesenteric, epididymal, and brown), small and large intestines, skeletal muscle and brains were collected, weighed, and snap frozen. HFD mice showed not only increases in liver weight but were noticeably larger and visibly steatotic as compared to their LFD fed controls (Figure 4). HFD increased liver weight by approximately $140.0 \%$ as compared to LFD mice (Figure 3B). Liver weights of HFD+MSX0.5\% fed mice were approximately $4.378 \mathrm{~g} \pm 0.30 \mathrm{~g}$ whereas $\mathrm{HFD}+\mathrm{MSX} 0.05 \%$ mice weighed just as much as HFD control mice ( $3.156 \mathrm{~g} \pm 0.44 \mathrm{~g}$ vs. $3.052 \mathrm{~g} \pm 0.22 \mathrm{~g}$ respectively). That trend also held true when comparing LFD+MSX 0.5\% (1.223g $\pm 0.03 \mathrm{~g})$ and LFD+MSX $0.05 \%(1.239 \mathrm{~g} \pm 0.06 \mathrm{~g})$ to LFD controls which weighed approximately $1.27 \mathrm{~g} \pm 0.02 \mathrm{~g}$. White adipose tissue (WAT) was also weighed and found to be significantly greater in HFD fed mice as compared to LFD mice at 2.190 $\mathrm{g} \pm 0.10 \mathrm{~g}$ and $1.398 \mathrm{~g} \pm 0.19 \mathrm{~g}$ respectively (Figure $3 \mathrm{C}$ ). A decrease in WAT weight was only observed in the LFD+MSX $0.5 \%$ fed mice by approximately $19.5 \%(1.125 \mathrm{~g} \pm 0.11 \mathrm{~g})$ as compared to the LFD control diet fed mice $(1.398 \mathrm{~g} \pm 0.19 \mathrm{~g})$.

Serum concentrations of pro-inflammatory cytokine, IL-6, were quantified by ELISA. IL-6 concentrations were found to be significantly increased in HFD fed mice $(125.7 \mathrm{pg} / \mathrm{ml} \pm$ $7.01 \mathrm{pg} / \mathrm{ml}$ ) as compared to LFD controls $(107.4 \mathrm{pg} / \mathrm{ml} \pm 1.46 \mathrm{pg} / \mathrm{ml})$. Moreover, IL-6 levels were significantly abrogated in HFD mice supplemented with MSX at $0.5 \%(106.8 \mathrm{pg} / \mathrm{ml} \pm 2.40 \mathrm{pg} / \mathrm{ml}$ ) and $0.05 \%(111.7 \mathrm{pg} / \mathrm{ml} \pm 1.39 \mathrm{pg} / \mathrm{ml})$ 


\section{MSX supplementation improves glucose sensitivity in HFD mice}

This HFD in particular is designed to model the chronic consumption of high fat, high sugar foods common to those found in the American obese population. To confirm the phenotype, Glucose sensitivity was measured during Week 12 using a Glucose Tolerance Test (GTT). The results indicated that HFD mice have become less sensitive to glucose as compared to the LFD fed mice(Figure 1). Despite MSX's inability to significantly lower Body Weight, sensitivity to glucose was significantly affected. The most glucose sensitive group following the 6 hour fast is the LMSX0.05\% group (AUC of $19529.8 \pm 3613$ SEM). The addition of MSX to the HFD at high doses (i.e. $0.5 \% \mathrm{w} / \mathrm{w}$ ) appears to have increased AUC levels albeit slightly (AUC of $64177.3 \pm$ 3994 ) as compared to HFD alone (AUC of $55874 \pm 4466$ ). Following a two week resting period, we performed an insulin tolerance test (ITT) to measure the impact of the ALIOS diet on Insulin action following a 6 hour fast (Figure 2). Once again, HFD mice displayed higher tolerances to insulin as compared to their LFD alone counterparts with AUC levels of $9964 \pm 1522$ and $5282 \pm$ 377.1 respectively. MSX however did not have a significant effect in improving sensitivity to insulin across all diet types and doses of MSX.

\section{Tissue Histology confirms ALIOS diet-induced necrosis, lipid accumulation, and inflammation}

H\&E staining of liver sections showed the influence of the ALIOS diet on inducing a NASH/NAFLD phenotype within an 18 week period. Lipid Accumulation, Necrosis, Inflammation and Biliary Hyperplasia were scored from 0-5 based on severity (Table 1 and 2). Mice administered the ALIOS diet showed significant levels of biliary hyperplasia and lipid accumulation as compared to LFD treated animals. In the HFD cohort, MSX at $0.5 \%$ or $0.05 \%$ did not significantly improve severity or NAS scoring in any measure. 
HFD causes an increase in liver fibrosis and apoptosis proteins and decreases antioxidant/hormone catabolism enzymes and lipid transport, metabolism and catabolism proteins

Liver proteomic analysis of mouse livers from the high fat diet control group demonstrated significant modulation in a number of NASH/NAFLD associated proteins. Bottomup analysis of approximately 1300 proteins from each liver was filtered to approximately 33 statistically significant proteins. Fold change as compared to the low fat diet group was then calculated and visualized in a functionally grouped heatmap (Figure 6). Volcano plots were also utilized to further make comparisons between each treatment group. Of the statistically significant proteins identified, CYP2C29, Hal, CYP1A2, CYP2C50, CSAD, Ftl1 and Abat were downregulated in HFD livers as compared to LFD controls(Figure 7). Consequently, Aldh3A2, Anxa5, Ehhadh, Scp2, Vim, Plin2, Glul, Fabp2, Apoe1, Decr2, Fgb and GK were upregulated compared to LFD mice(Figure 7). The addition of MSX to the high fat diet augmented protein levels of Anxa5 and Arhgdia for both $0.5 \%$ and $0.05 \%$ doses as compared to HFD control livers (Figure 8 and 9). HFD+MSX0.05\% showed significantly high levels of Anxa5 and Arhgdia as compared to HFD controls (Figure 9). Significant reductions in LFD mice supplemented with MSX at $0.5 \%$ w/w, Aldh3a2, Acot2, Scp2, Decr2, Acaa1a, Apoe, Fabp1, , Plin2, Fabp2, and Apoa1 were observed as compared to LFD control mice(Figure 10). Of note, a lack of statistically significant changes in LFD+MSX0.05\% livers was also noted (Figure 11). 


\section{MSX abrogates LPS stimulated pro-inflammatory cytokine release in healthy human donor monocytes and PMA differentiated THP-1 Macrophages}

In a preliminary experiment, healthy human donor monocytes were pretreated with various concentrations of MSX prior to exposure to LPS for 23 hours. LPS exposure alone increased the secretion of pro-inflammatory cytokine IL- 6 by nearly 600 fold as compared to vehicle, DMSO, control (983.8 pg/ml \pm 6.56 vs. $1.64 \mathrm{pg} / \mathrm{ml} \pm 0.52$ respectively)(Figure 12A). TNF $\alpha$, which was undetectable in vehicle control, was significantly abundant in media of LPS treated monocytes at approximately $212 \mathrm{pg} / \mathrm{ml} \pm 4.35 \mathrm{pg} / \mathrm{ml}$ )(Figure 12B). While MSX at doses of 10,50 , and $100 \mu \mathrm{g} / \mathrm{ml}$ significantly decreased IL-6 media concentrations, it was only at 50 and $100 \mu \mathrm{g} / \mathrm{ml}$ of MSX that were able to significantly reduce the release of TNF $\alpha$ into media by $11.9 \%(186.6 \mathrm{pg} / \mathrm{ml} \pm 3.8 \mathrm{pg} / \mathrm{ml})$ and $84.8 \%(32.14 \mathrm{pg} / \mathrm{ml} \pm 1.04 \mathrm{pg} / \mathrm{ml})$ respectively.

Resveratrol (RESV) at a dose of $20 \mu \mathrm{M}$ was used as to compare the effects of MSX to a real world highly studied polyphenol. IL-6 and TNF $\alpha$ were both significantly reduced by approximately $51.9 \%(473 \mathrm{pg} / \mathrm{ml} \pm 1.79 \mathrm{pg} / \mathrm{ml})$ and $77.6 \%(47.51 \mathrm{pg} / \mathrm{ml} \pm 0.08 \mathrm{pg} / \mathrm{ml})$ respectively.

To support this finding, THP-1 monocytes were cultured and differentiated into macrophages using PMA. As mentioned earlier, differentiated THP-1s were pre-treated with vehicle or various doses of MSX for at least 1 hour before the addition of $1 \mu \mathrm{g} / \mathrm{ml}$ of LPS into cell culture media for 6 or 24 hours. As seen in the human donor samples, LPS exposure alone significantly stimulated the release of IL- 6 by 4.6 fold at 6 hours (Figure 13A) and 177.5 fold at 24 hours (Figure 13C) as compared to the vehicle alone control. TNF $\alpha$ concentrations in LPS only treated cells showed similar levels of augmentation at 6 (Figure 13B) and 24hours (Figure 13D) at 207.3 fold and 147 fold respectively. 
The addition of MSX at $50 \mu \mathrm{g} / \mathrm{ml}$ afforded the most significant reduction of IL-6 at 6 hours by approximately $49 \%$ and by $17 \%$ after 24 hours as compared to LPS treated cells alone. Cells treated with MSX at a concentration of $50 \mu \mathrm{g} / \mathrm{ml}$, demonstrated the most significant reduction in TNF $\alpha$ by $33.4 \%$ after 6 hours as compared to LPS treated controls. After 24 hours, neither dose of 50 or $100 \mu \mathrm{g} / \mathrm{ml}$ were able to significantly reduce cytokine secretion into media as compared to LPS insulted cells. RESV at a concentration of $10 \mu \mathrm{M}$ was found to be only effective at significantly reducing IL- 6 secretion after 6 hours by approximately $16.7 \%$.

With the consistent observation that a reduction in cytokine release is only apparent at a lower dose than a higher dose, we sought to repeat this experiment but expand on the number of cytokines measured through multiplexing. Once more, THP-1 monocytes were seeded directly onto 24 well plates before being differentiated into macrophages. Cells were treated with vehicle alone, LPS alone or pretreated with $\operatorname{MSX}(0.1,1,10$ or $100 \mu \mathrm{g} / \mathrm{ml})$ or RESV (10 or $20 \mu \mathrm{M}$ ) for 1 hour prior to LPS exposure for another 5 hours. As compared to vehicle treated cells, concentrations of IL-6, TNF $\alpha$, IL-1 13, MCP-1, IL-1RA, IL-10 were significantly increased following LPS exposure by 15.6, 1519, 12.6, 16.9, 14.1 and 40.9 fold respectively (Figure 14). RESV (10 and $20 \mu \mathrm{M}$ ) was used again for comparison purposes and showed a statistically significant reduction in all secreted cytokines at both concentrations in all but one case (RESV at $10 \mu \mathrm{M}$ and TNF $\alpha$ ). TNF $\alpha$ and IL-10 concentrations in vehicle treated cells were not detectible. MSX at various doses were the most effective and reduced IL-6, TNF $\alpha$, IL-1 $\beta$, MCP-1, IL-1RA and IL-10 by $3.00(1 \mu \mathrm{g} / \mathrm{ml} \mathrm{MSX}), 2.20(0.1 \mu \mathrm{g} / \mathrm{ml}), 2.20(10 \mu \mathrm{g} / \mathrm{ml}), 4.32(100 \mu \mathrm{g} / \mathrm{ml}), 8.20$ $(0.1 \mu \mathrm{g} / \mathrm{ml}), 3.20(1 \mu \mathrm{g} / \mathrm{ml})$ fold respectively (Figure 14). The effects of MSX again at the lower doses (namely 0.1 and $1 \mu \mathrm{g} / \mathrm{ml}$ ) appeared to continue the trend that was observed in previous cell based experiments. 


\section{Discussion}

MSX was evaluated in a murine model of western diet-induced obesity for 18 weeks. In an effort to minimize variability mouse to mouse and from cage to cage, male mice were selected over female mice on two major considerations. First, we considered sex differences in weight regulation for an extended period of time ${ }^{36,37}$. Second, MSX is abundant in phytoestrogens which may play vastly different roles in male and female mice with regards to adiposity, glucose/insulin response, and the progression or severity of NAFLD ${ }^{38-41}$. To minimize cage to cage variability, bedding was collected, pooled within treatment groups, and redistributed with fresh bedding every two weeks ${ }^{21,26}$. This was done in an effort to address the cohousing effect which may be present in mice housed with two or more mice. Mice are corophagic and will consume fecal material under times of stress or fasting. Overtime, the microbes present in feces will alter the gut microbiome of each mouse potentially causing variability in response to each diet and treatment of MSX cage to cage $\mathrm{e}^{8,26}$.

The diet selected for this study was chosen to model the typical consumption of saturated fats and sugars common in western diets ${ }^{42,43}$. Moreover, this is compounded with the addition of cholesterol and high fructose/glucose water to model the intake of sugary drinks $^{8,44,45}$. Sedentary behavior, a necessary component in stimulating obesity in humans, is encouraged by placing food directly inside the cage as opposed to in the top mounted feeder.

This effectively translates to a gradual progression of NASH/NAFLD by 18 weeks, glucose/insulin intolerance by 12 and 15 weeks and peripheral inflammation by 18 weeks. We know that obesity is a disproportionate intake of high energy foods while expending fewer calories. Other diets such as a Methionine-Choline Deficient Diet (MCD), produce liver damage, body weight gain, and steatosis, the MCD diet does not produce insulin or glucose resistance nor does it induce a profound inflammatory response ${ }^{46}$. 
With a $45 \%$ kcal diet supplemented with cholesterol, a long term exposure ( $>12$ weeks) is needed to induce weight gain, insulin resistance but fructose is needed to induce hepatic steatosis, steatohepatitis and fibrosis ${ }^{8,27}$. Similar studies have also reported a mild to moderate NASH phenotype in 16 weeks with aged mice ${ }^{47}$. While MSX did not lower body weight at week 18 as in our previous 12 week pilot study, it is possible the effects of age, a more chemically diverse diet, and the manner in which MSX was formulated may have contributed to this observation. Differences in MSX production and formulation may have altered bioavailability and thus could have mitigated any beneficial effects as previously seen in our prior pilot study. In this study, MSX was directly encapsulated in casein before cold extrusion into pellet form by Teklad.

Our previous pilot study required fresh incorporation of MSX into ground food prior to administration to each cage. Moreover, the constituents of MSX, which are found in heat intensive process of developing pure maple syrup, are thermostable and are unlikely to have spontaneously degraded at room temperature. The formulation and preparation of MSX may have also effected the dosing each mouse received which may explain why mice in this study did not show significant reductions in body weight at week 18. Despite a lack of body weight loss, we observed WAT weights were significantly reduced in mice fed LFD with $0.05 \%$ MSX (as compared to LFD control diet fed mice).

Timing and duration of this study was expanded from the initially proposed 12 weeks to 18 weeks based on findings from two attempts of i.p. glucose and insulin tolerance tests. We concluded that mice fed a HFD were glucose intolerant by week 12 and showed intolerance to insulin by week 15. Fasting blood glucose was also measured and found to be elevated in HFD mice by week 8 . 
HFD+MSX0.5\% fed mice showed a decreasing, although non-statistically significant, trend in improving insulin sensitivity as compared to HFD control fed mice. LFD mice fed MSX, however showed worse sensitivity to insulin despite an improvement in glucose tolerance at week 12 as compared to LFD control mice. MSX at a dose of $0.05 \%$ in HFD mice showed a similar decreasing trend towards increasing glucose sensitivity as compared to HFD control diet fed mice. Only LFD fed mice with MSX 0.05\%, showed significantly improved glucose sensitivity. Taken together, Insulin resistance and fasting hyperglycemia, which are hallmarks of MetS, T2DM, and NASH, were observed in these mice fed the ALIOS diet. Tissue histology of mice sacrificed at week 18 confirmed that a NASH and NAFLD phenotype was present based on scoring for disease specific markers such as inflammation, biliary hyperplasia, necrosis, and steatosis. When compared to our previous pilot study, MSX supplementation at the same dose (0.5\%) appeared to augment steatosis and exacerbate the NASH phenotype in ALIOS diet fed mice as evidenced by H\&E histology scoring. In an 8 week study, Kamei et al showed through transcriptomic analysis of a similar 45\% kcal study using identically aged male C57BL/6 mice but with their formulation of a maple syrup extract at a dose of $0.06 \% \mathrm{w} / \mathrm{w}$, the rate limiting step in triacylglyceride synthesis, ACOD1 was found to be significantly upregulated in HFD+MSX mice despite noting a decrease in liver TG levels, an increase in serum TG levels, and no appreciable decrease in liver and body weight as compared to HFD control mice. Kamei also reported that maple syrup extract maintained liver health through immunomodulation or significant downregulation in C-X-C motif ligand genes and Serum Amyloid A isoform genes as compared to HFD control mice. These findings prompted our group to investigate the physical hepatic protein levels using an unbiased, state-of- the- art, mass spectrometry based method to relatively quantify relevant hepatic proteins. 
Proteomic analysis of HFD livers compared to LFD control mice revealed an increase in fibrosis/clot forming proteins (Fibrinogen $\beta$ (Fibb), Vimentin (Vim)), apoptosis regulators (Annexin 5 (Anxa5), Apoptosis Inducing Factor 1, mitochondrial (AIFM-1)) ${ }^{48-50}$. Moreover, peroxisomal lipid metabolism proteins (Decr2 and Ehhadh), lipid transport proteins (Fatty acid bind protein 2 (Fabp 2), sterol carrier proetin 2 (Scp2)), Fatty Acid metabolism proteins (Fatty aldehyde dehydrogenase (Aldh3a2), Apolipoprotein a1 (ApoA1)), and Glycerolkinase (GK), a key enzyme in fructose metabolism and triglyceride synthesis were also increased ${ }^{51-55}$. Interestingly, MSX increased the abundance of Anxa5 and Aifm 1 significantly at both $0.5 \%$ and even more so at $0.05 \%$ as compared to HFD controls. LFD mice with MSX showed no aberrant levels of Anxa5 or Aifm1.

A Rho GDP Dissociation Inhibitory protein (Arhgdia), which, when decreased is implicated in metastatic and aggressive hepatocellular carcinoma, was indeed decreased in HFD control mice but significantly increased in both HFD+MSX0.5\% and HFD+MSX0.05\% cohorts indicating a possible anti-carcinogenic mechanism ${ }^{56}$.

Enzymes involved in lipid particle removal/catabolsim (Perilipin 2 (Plin2)) and amino acid metabolism were also increased in HFD fed mice such as Glutamine synthetase (Glul) (Glutamate metabolism and ammonia removal) $)^{57,58}$. Conversely, Taurine biosynthesis associated enzyme, Cysteine sulfinic acid decarboxylase (CSAD) and Histidine Ammonia Lyase (Hal) were markedly decreased in HFD mice compared to LFD controls however, HFD+MSX0.5\% livers showed even greater deficiencies in these proteins. 
Several cytochrome P450 enzymes (CYP1A2, 2C50, 2C29, 4A12A) which were found to be involved in hormone catabolism, fatty acid/xenobiotic detoxification, and steroidogenesis were also significantly abrogated in these HFD mice compared to LFD controls ${ }^{59-62}$. Moreover these CYP enzymes were significantly downregulated in MSX supplemented HFD mice at both doses. It is still unclear role these specific enzymes play in tandem within the progression of NASH/NAFLD however, a decrease in enzymes responsible for the removal of potentially oncogenic or DNA damaging endogenous (or exogenous) species may play a significant role in hepatic neoplasias including hepatocellular carcinomas warranting future study ${ }^{59}$.

Disregulation in Iron homeostasis is associated with NASH to NAFLD onset and progression $^{63}$. Ferritin, specifically ferritin light chain 1 (Ftl1), was significantly decreased in HFD control mice with no appreciable effect seen from MSX supplementation. Finally, a key GABA metabolizing protein, Abat, which is critical for proper glucose homeostasis, food intake and body weight, was found to be significantly downregulated in HFD control mice and even further downregulated in both MSX supplemented cohorts ${ }^{64,65}$. LFD+MSX0.5\% mouse livers also confirmed this reduction despite no statistical significance. All in all, proteomic analyses of these livers show that MSX may augment certain processes in liver lipid accumulation and hepatocyte damage/death. These findings further prompted us to ask, why was MSX harmful in this murine study but beneficial in our pilot study? With mouse age and the core diet being identical to our previous pilot study, one key difference could lie in the addition of fructose to our HFD fed mice. 
The addition of fructose and glucose in drinking water for this 18 week study could have further exacerbated these NASH/NAFLD symptoms and masked any beneficial effects of MSX. Fructose is largely metabolized in the liver via a non-insulin dependent manner which leads to hepatocellular depletion of ATP and thus an increase in both ADP and uric acid ${ }^{66,67}$. The generation of this acid leads to oxidative stress, necrosis, and eventually apoptosis of hepatocytes. Insulin resistance leads to not only hyperinsulinemia and hyperglycemia but de novo lipogenesis ${ }^{66,67}$. The metabolism of fructose combined with an influx of dietary cholesterol leads to de novo lipogenesis, enhanced generation of triacylglyceride precursors, and further exacerbates hepatic oxidative stress ${ }^{9,51,66,67}$. The influence of fructose on lipogenesis in the liver, combined with MSX's inhibition of key lipid and fructose metabolizing enzymes may explain the augmented steatotic and necrotic effects in the liver.

The actual gut microbial metabolites of MSX have yet to be identified.

Secoisolariciresinol (SECO), a significant MSX constituent, is hydrolyzed and metabolized by the gut microbiota to Enterodiol (ED) and Enterolactone $(E L)^{68}$. In silico and in vitro permeability assays by our group have shown that Enterolactone is most likely to passively permeate through the intestinal lining (data not shown). More importantly, EL has also been shown by others to have differential effects in high fat diet fed mice and hepatocyte cultures. In one instance, the (-)(levorotatory) stereoisomer of EL ameliorates the effects of a high fat diet in mice $^{69}$. On the other hand, a racemic mixture or even the $(+)$ (dextrorotatory) EL can cause steatosis by down regulating PPAR $\alpha$, CPT1, pAMPK and increasing expression of SREBP1 ${ }^{70}$. EL was also found to promote extracellular efflux of fatty acids but also inhibited Beta oxidation and intracellular lipid metabolism leading to an increase in lipid droplet accumulation, altered lipid composition, an increase in pro-inflammatory cytokines and hepatocyte death ${ }^{70,71}$. 
Taken together, a thorough understanding of the biologically relevant gut microbial metabolites of MSX is needed to fully assess the impact of the other potentially beneficial polyphenol classes within MSX.

Initial in vitro experiments sought to translate findings from murine macrophage systems to a more appropriate human model. In this case, Human donor monocytes were cultured and exposed to LPS and increased two key pro-inflammatory cytokines: IL-6 and TNF $\alpha$. By pre-treating these cells with MSX at various non-toxic doses and evaluating their immunomodulatory role alongside a commonly studied polyphenol, Resveratrol (RESV). Healthy human donor monocytes responded to LPS stimulation for 24 hours by secreting significant quantities of IL-6 and TNF $\alpha$ as compared to vehicle control (DMSO $<0.1 \%$ ). Moreover, Resveratrol was also able significantly decrease the release of these cytokines into culture medium. MSX also displayed the ability to significantly modulate the release of these cytokines at the lowest tested dose of $10 \mu \mathrm{g} / \mathrm{ml}$.

Given that a major limitation of using human donor monocytes is variability lot to lot, Immortalized THP-1 human monocytes where used to reproduce these findings. THP-1 immortalized monocytes are often used to study the immunomodulatory properties of a drug or natural product/extract ${ }^{72,73}$. Moreover they may be co-cultured with other cell types to study the regulatory mechanisms associated with cytokine/chemokine secretion in various tissues ${ }^{72,73}$. In order to sample a broader range and quantity of cytokines in this system, PMA was used to differentiate these monocytes to macrophages.

Following the previously described procedure, the immunomodulatory effects of MSX and a comparator compound, RESV, were assayed over the course of 6 and 24 hours. As performed previously, differentiated THP-1 cells were exposed to vehicle, MSX or RESV for 1 hour before LPS (at $1 \mu \mathrm{g} / \mathrm{mL}$ ) addition. 
As seen in Figure 13, LPS significantly increased IL-6 and TNF $\alpha$ release as compared to vehicle control after 6 hours however; IL- 6 and TNFa concentrations were diminished by 24 hours. RESV at $10 \mu \mathrm{M}$ decreased IL-6 significantly as compared to LPS control wells. More importantly, MSX decreased IL- 6 and TNF $\alpha$ media concentrations at 6 hours. This data allowed us to confirm the ideal timepoints of quantifying cytokines in the THP-1 system as also observed elsewhere ${ }^{73}$. Therefore, we repeated this experiment again with the goal of sampling more pro and anti-inflammatory cytokines using the Procarta Plex Multiplex.

Again as with previous experiments, THP-1 monocytes were differentiated using PMA and pretreated with either vehicle, RESV $(10$ or $20 \mu \mathrm{M})$ or $\operatorname{MSX}(0.1,1,10$, or $100 \mu \mathrm{g} / \mathrm{mL})$ for 1 hour prior to LPS (at $1 \mu \mathrm{g} / \mathrm{mL}$ ) addition. LPS significantly increased all measured cytokines as compared to vehicle control. Similarly, Resveratrol (particularly at $20 \mu \mathrm{M}$ ) significantly reduced the release of these pro-inflammatory cytokines. Interestingly, MSX at lower concentrations < $10 \mu \mathrm{g} / \mathrm{mL}$ modulated the release of all cytokines except IL-1 $\beta$.

This may be substantiated simply due to the timing of when these media samples were removed from culture. IL-1 $\beta$ is an initiator and early signaling molecule of the inflammasome with a sorter half-life as compared to IL-6 or TNF $\alpha^{74,75}$. Moreover, the cytokine IL-1R receptor Antagonist is a direct inhibitor of IL-1 13 and expressed at the same time under the Toll-Like Receptor Myd88 dependent MAPK-ERK pathway ${ }^{76}$. While no direct mechanism of action studies were performed using MSX, we may infer from the Procarta Plex cytokine data that PMA differentiated and LPS stimulated THP-1 macrophages secreted cytokines via the CD14/TLR4 mediated MyD88 dependent pathway. 
This pathway includes MAPK and ERK along with AP-1 which may translocate into the nucleus to directly bind to pro-inflammatory response elements leading to the production of not only pro-inflammatory molecules (IL-1 $\beta$, IL-6, TNF $\alpha$, and MCP-1) but also their anti-inflammatory counterparts (IL-10, IL-1RA). Selective inhibition of these targets with representative compounds of MSX may elucidate a mechanism of action in macrophages. 


\section{Conclusions}

Initially, we hypothesized that MSX prevented metabolic syndrome through its antiinflammatory or immunomodulatory effects. In a LPS stimulated immortalized human macrophage model, MSX was able to potently abrogate the secretion of pro-inflammatory cytokines (IL-6, TNF $\alpha$, IL-1 $\beta$, and MCP-1). With much of the LPS signaling pathway known in THP2 macrophages, we can only infer that MSX engages the TLR4-MAPK-ERK-NfkB pathway. With such a profound effect on inflammation in vitro, we sought to not only translate these findings into another mammalian model but replicate our previous in vivo findings. To do so we used a diet-induced obesity murine model that mimicked lifestyle and consumption risk factors found in the United States such as excessive intake of saturated fat, cholesterol and fructose combined with sedentary behavior. This western diet allowed us to truly examine MSX's ability to prevent the onset and/or progression of metabolic syndrome and NASH/NAFLD.

Our current findings suggest that after 18 weeks of dietary exposure to a high fat -high fructose, western style diet, that MSX does not prevent or deescalate the symptoms associated with metabolic syndrome and fatty liver disease as supported by necropsy data , liver histology and liver proteomics. Despite reducing serum levels of pro-inflammatory IL-6, additional in vivo studies with a LFD to HFD switch with and without MSX should be performed to further evaluate the beneficial potential of this polyphenol enriched extract. 


\section{Funding Acknowledgements}

This study was supported through a grant from the National Institute of Food and Agriculture (NIFA) and USDA Grant \# 2017-67017-26784, Project \# RI.W-2016-08934, Accession \# 1011820 and matching funds from the Quebec Maple Syrup Producers (QMSP).

\section{Author Contributions}

NAD wrote the manuscript, designed, conducted, and analyzed all monocyte/macrophage cell based assays. NAD, MP, JA, EM, HM, CL, KR, SL, and WW maintained and sacrificed animals. BB performed liver homogenization and liver proteomic analysis. AS and NPS conceived the overall study and edited the manuscript.

\section{Acknowledgments}

Hepatic histopatholgy scoring was performed by Dr. Michael Goedken, D.V.M., Ph.D., Diplomate ACVP of Rutgers University.

Conflict of Interest

The authors of this manuscript declare no financial conflict of interest 
Figures and Captions

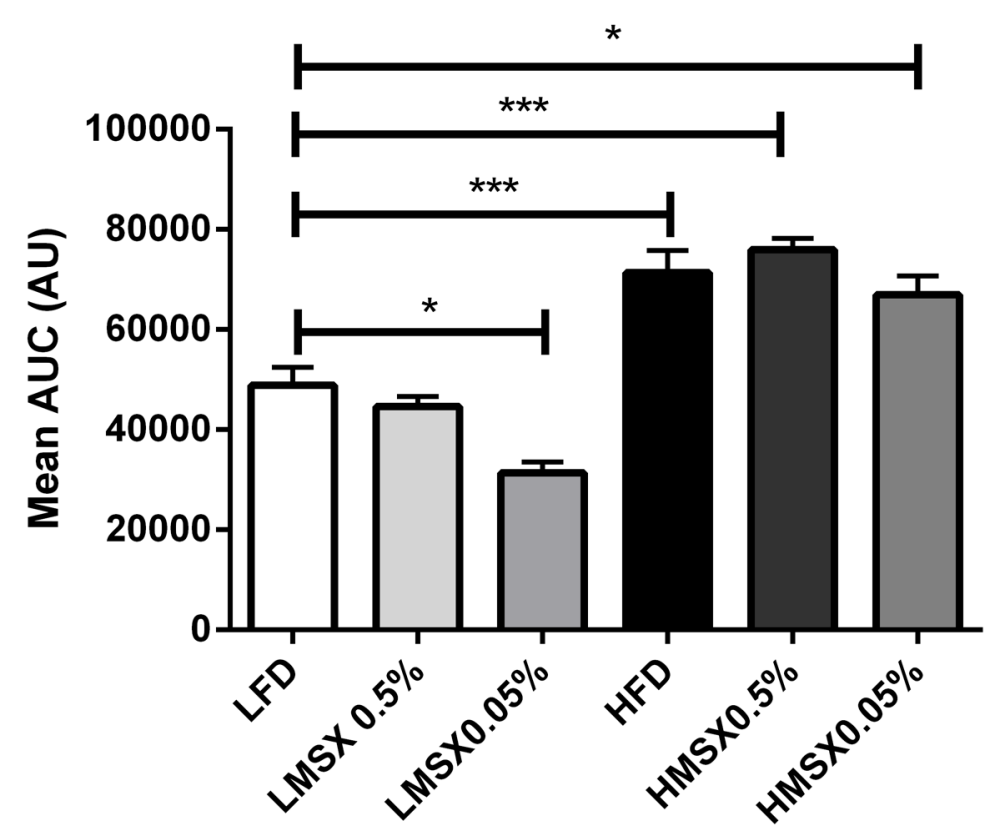

Figure 1.) Week 12 Glucose Tolerance Test (GTT) shows greater insensitivity to Glucose in HFD mice as compared to LFD mice. Mice fed a HFD returned totheir inital fasting blood glucose significantly longer as compared to mice fed a LFD. Inclusion of MSX at $0.05 \% \mathrm{w} / \mathrm{w}$ in LFD mice also significanly improved sensitivity to glucose as compared nonsupplemented LFD fed mice. All data shown as Mean AUC \pm SE with all comparisions made to the LFD where $p<0.05^{*}, p<$ $0.01 * *, \mathrm{p}<0.001 * * *, \mathrm{p}<0.0001 * * * *$. 


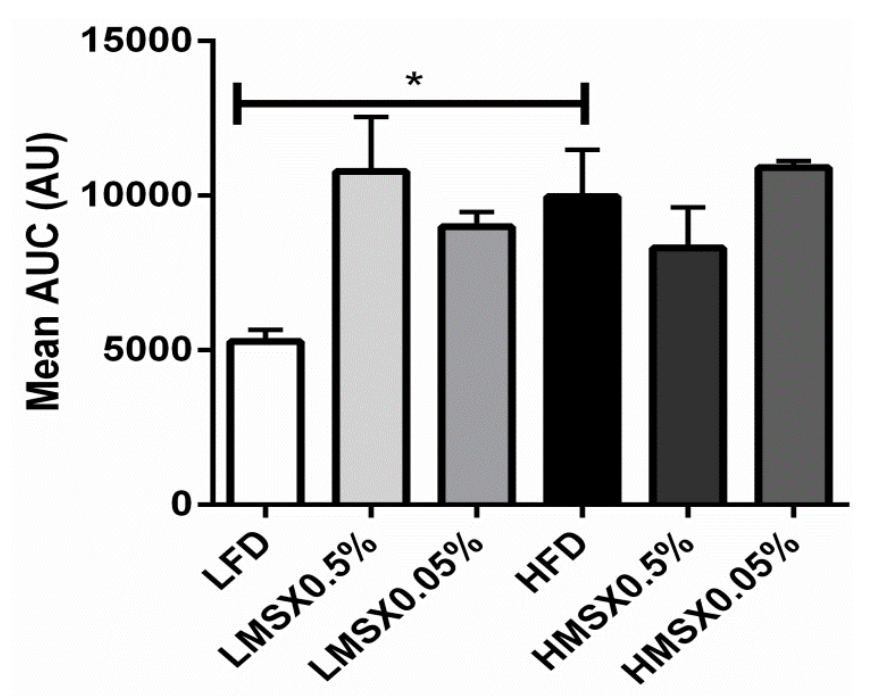

Figure 2.) Week 15 Insulin Tolerance Test (ITT) shows greater insensitivity to Insulin in HFD mice as compared to LFD mice. Mice fed a HFD returned totheir inital fasting blood glucose significantly longer following a bolus of insulin $(0.75 \mathrm{U} / \mathrm{kg})$ after a 6 hour fast as compared to mice fed a LFD. Inclusion of MSX at any concentration did not signifcantly improve sensitivity to insulin within any diet. All data shown as Mean AUC \pm SE with all comparisions made to the LFD where $\mathrm{p}<0.05^{*}, \mathrm{p}<0.01 * *, \mathrm{p}<0.001 * * *, \mathrm{p}<0.0001 * * * *$. 

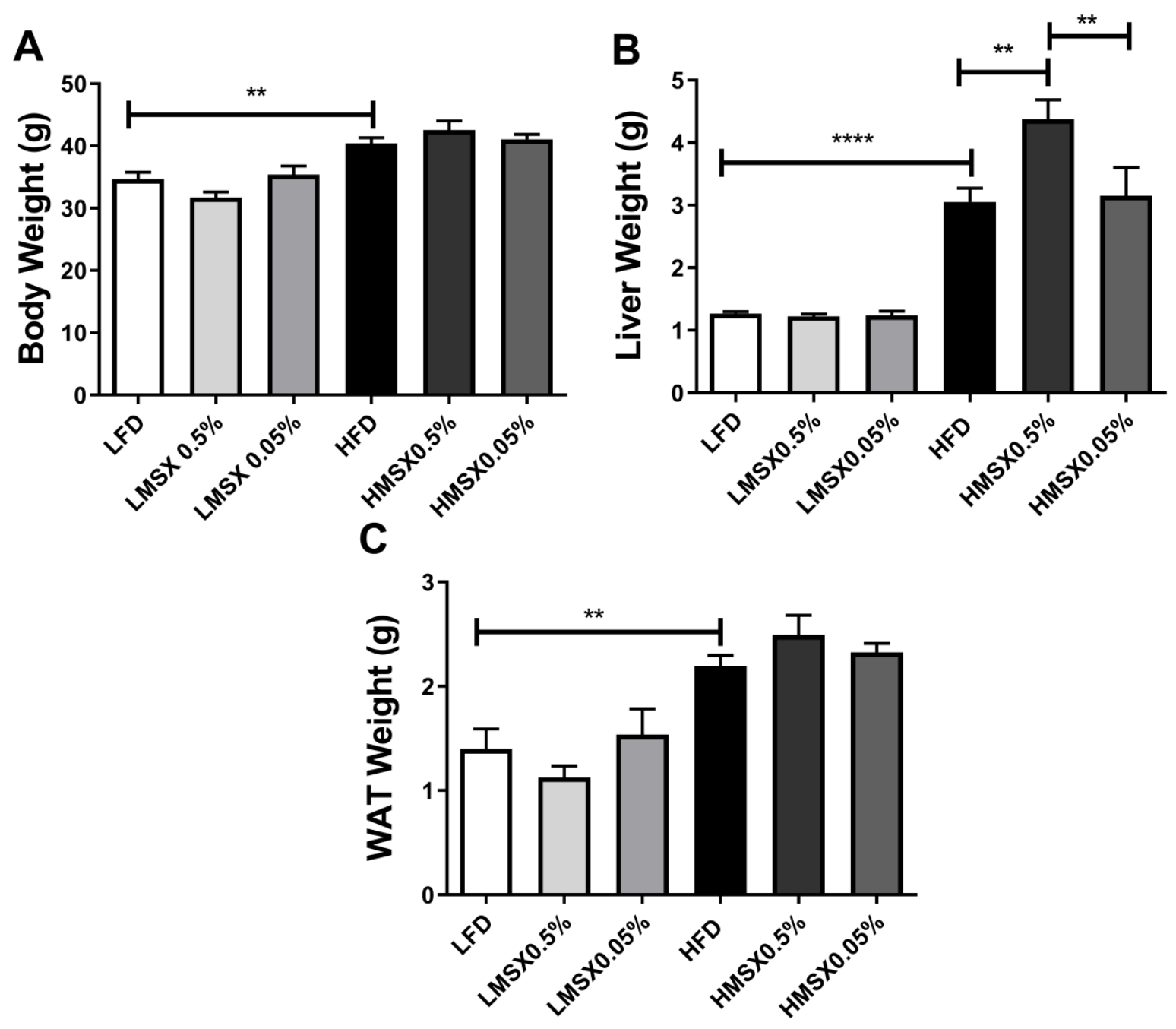

Figure 3.) Week 18 Necropsy Body Weight, Liver, and White Adipose Tissues Weights. Final body and tissue weights following 18 weeks of western diet exposure were significantly increased as compared to LFD control mice. MSX0.5\% appeared to decrease body and WAT weight when added to LFD chow but augmented Liver and WAT in HFD feed as compared to their repective diet controls. Statistical significance was calculated using one way ANOVA where $p<0.05^{*}, p<0.01 * *, p<0.001^{* * *}, p<0.0001^{* * *}$ 


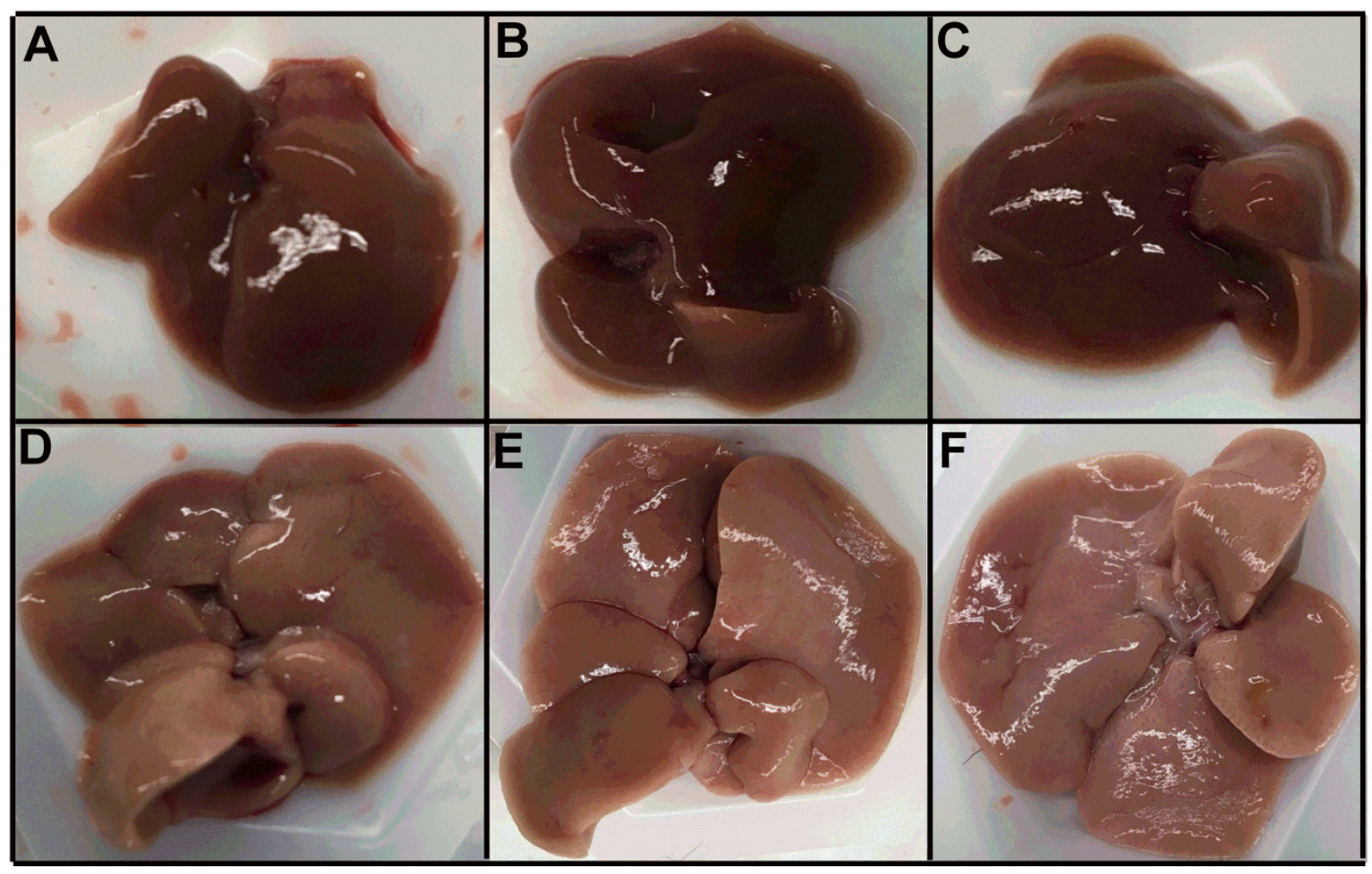

Figure 4.) Whole Liver comparisons between Low Fat Diet mice and High Fat diet mice with and without MSX supplementation. Representative images from each treatment group were taken to compare gross physiologic changes during the 18 week diet exposure timeline. Low Fat Diet (LFD) (A), LFD+MSX0.5\% (B), LFD+MSX0.05\% (C), HFD (D), HFD+MSX0.5\% (E), HFD+MSX0.05\% (F). 


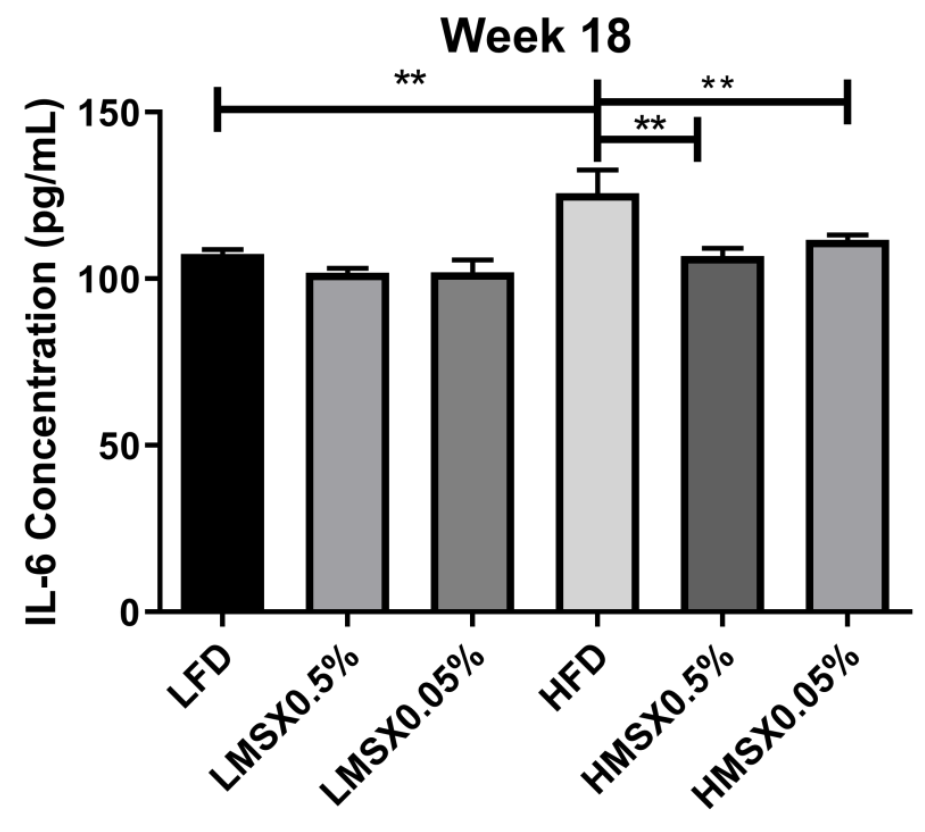

Figure 5.) Serum concentrations of pro-inflammatory cytokine IL-6 are elevated in HFD mice but abrogated by MSX at Week 18. Western diet feeding for 18 weeks afforeded a significant increase in pro-inflammatory IL-6 serum concentrations as compared to LFD controls. The addition of MSX at both $0.5 \% \mathrm{w} / \mathrm{w}$ and $0.05 \% \mathrm{w} / \mathrm{w}$ to the HFD, caused a significant abrogation in IL-6 as compared to HFD control mice.Statistical significance was calculated using one way ANOVA where $\mathrm{p}<0.05^{*}, \mathrm{p}<0.01^{* *}, \mathrm{p}<0.001^{* * *}, \mathrm{p}<0.0001^{* * * *}$, or not statistically significant (n.s). 


\begin{tabular}{|c|c|c|c|c|c|c|c|}
\hline \multicolumn{8}{|c|}{ Week 18 Histology Scoring Frequencies } \\
\hline & $\begin{array}{c}\text { Grade } \\
\text { (Severity \%) }\end{array}$ & $\begin{array}{l}\overline{3} \\
\stackrel{\infty}{0} \\
\stackrel{\infty}{0}\end{array}$ & 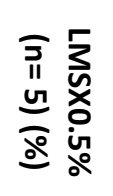 & 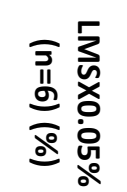 & 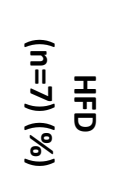 & 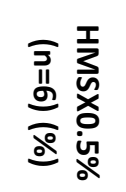 & 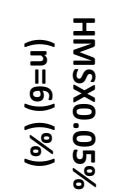 \\
\hline \multirow{6}{*}{$\begin{array}{c}\text { Lipid } \\
\text { Accumulation }\end{array}$} & $0(0)$ & $8(100)$ & $4(80)$ & 1 (16.6) & - & - & - \\
\hline & $1(<10)$ & - & $1(20)$ & $5(83.3)$ & - & - & - \\
\hline & $2(10-25)$ & - & - & - & - & - & - \\
\hline & $3(25-40)$ & - & - & - & - & - & - \\
\hline & $4(40-50)$ & - & - & - & $5(71.4)$ & 1 (16.6) & $3(50)$ \\
\hline & $5(>50)$ & - & - & - & $2(28.5)$ & $5(83.3)$ & $3(50)$ \\
\hline \multirow{6}{*}{ Necrosis } & $0(0)$ & $3(37.5)$ & $4(80)$ & $5(83.3)$ & $5(71.4)$ & $2(33.3)$ & $3(50)$ \\
\hline & $1(<10)$ & $5(62.5)$ & $1(20)$ & 1 (16.6) & $2(28.5)$ & $4(66.6)$ & $3(50)$ \\
\hline & $2(10-25)$ & - & - & - & - & - & - \\
\hline & $3(25-40)$ & - & - & - & - & - & - \\
\hline & $4(40-50)$ & - & - & - & - & - & - \\
\hline & $5(>50)$ & - & - & - & - & - & - \\
\hline \multirow{6}{*}{ Inflammation } & $0(0)$ & $2(25)$ & $3(60)$ & $5(83.3)$ & $2(28.5)$ & - & - \\
\hline & $1(<10)$ & $6(75)$ & $2(40)$ & 1 (16.6) & $4(57.2)$ & $4(66.6)$ & $4(66.6)$ \\
\hline & $2(10-25)$ & - & - & - & 1 (14.3) & $2(33.3)$ & $2(33.3)$ \\
\hline & $3(25-40)$ & - & - & - & - & - & - \\
\hline & $4(40-50)$ & - & - & - & - & - & - \\
\hline & $5(>50)$ & - & - & - & - & - & - \\
\hline \multirow{6}{*}{$\begin{array}{c}\text { Biliary } \\
\text { Hyperplasia }\end{array}$} & $0(0)$ & $8(100)$ & $3(60)$ & $5(83.3)$ & - & - & - \\
\hline & $1(<10)$ & - & $2(40)$ & 1 (16.6) & $7(100)$ & $3(50)$ & $4(66.6)$ \\
\hline & $2(10-25)$ & - & - & - & - & $3(50)$ & $2(33.3)$ \\
\hline & $3(25-40)$ & - & - & - & - & - & - \\
\hline & $4(40-50)$ & - & - & - & - & - & - \\
\hline & $5(>50)$ & - & - & - & - & - & - \\
\hline \multirow[b]{2}{*}{ Tumor } & 0 (Absent) & $7(87.5)$ & $5(100)$ & $5(83.3)$ & $7(100)$ & $6(100)$ & $5(83.3)$ \\
\hline & 1 (Present) & 1 (12.5) & - & 1 (16.6) & - & - & 1 (16.6) \\
\hline
\end{tabular}

Table 1.) Week 18 Histology Scoring Frequencies 


\begin{tabular}{|c|c|c|c|c|c|}
\hline \multicolumn{6}{|c|}{ Week 18 Histology P Values } \\
\hline Feature & $\begin{array}{c}\text { LFD } \\
\text { Vs. } \\
\text { HFD } \\
\end{array}$ & $\begin{array}{c}\text { LFD } \\
\text { vs. } \\
\text { LMSX0.5\% } \\
\end{array}$ & $\begin{array}{c}\text { LFD } \\
\text { vs. } \\
\text { LMSX0.05\% }\end{array}$ & $\begin{array}{c}\text { HFD } \\
\text { Vs } \\
\text { HMSX0.5\% }\end{array}$ & $\begin{array}{c}\text { HFD } \\
\text { vs. } \\
\text { HMSX0.05\% } \\
\end{array}$ \\
\hline $\begin{array}{c}\text { Lipid } \\
\text { Accumulation }\end{array}$ & $\begin{array}{c}* * * \\
0.002\end{array}$ & 0.3846 & $\begin{array}{c}* * \\
0.003\end{array}$ & 0.1026 & 0.5921 \\
\hline Necrosis & 0.3147 & 0.2657 & 0.1375 & 0.2861 & 0.5921 \\
\hline Inflammation & $>0.9999$ & 0.2929 & 0.1026 & 0.2937 & 0.2937 \\
\hline $\begin{array}{c}\text { Biliary } \\
\text { Hyperplasia }\end{array}$ & $\begin{array}{c}* * * \\
0.0002\end{array}$ & 0.1282 & 0.4286 & 0.0699 & 0.1923 \\
\hline Tumor & $>0.9999$ & $>0.9999$ & $>0.9999$ & $>0.9999$ & 0.4286 \\
\hline
\end{tabular}

Table 2.) Week 18 Histology Scoring P-values. Individual Comparisons between each treatment group were made from rank ordered scores derived from the Mann-Whitney Non-parametric ttest where $\mathrm{p}<0.05^{*},<0.01 * *,<0.001 * * *,<0.0001 * * * *$. 


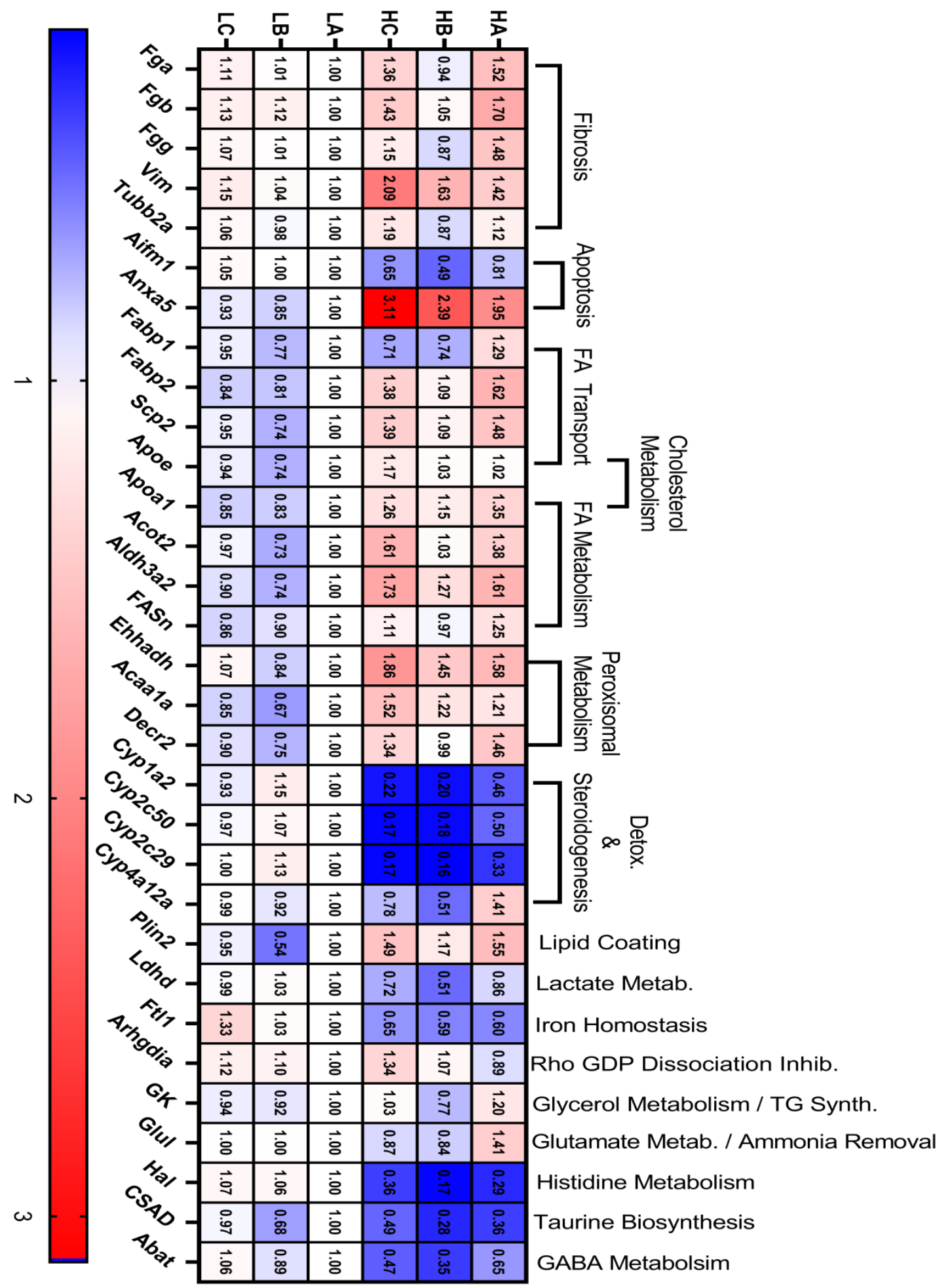

Figure 6.) Proteomic Analysis of Week 18 Livers reveal significant increases in NASH/NAFLD associated proteins including markers of fibrosis, apoptosis, and lipid regulation. HFD (HA), HFD+MSX0.5\% (HB), HFD+MSX0.05\% (HC), LFD (LA), LFD+MSX0.5\%(LB), LFD+MSX0.05\% (LC). Values shown as fold change compared to LFD group $(n=5)$ 


\section{Volcano Plot (HFD vs. LFD)}

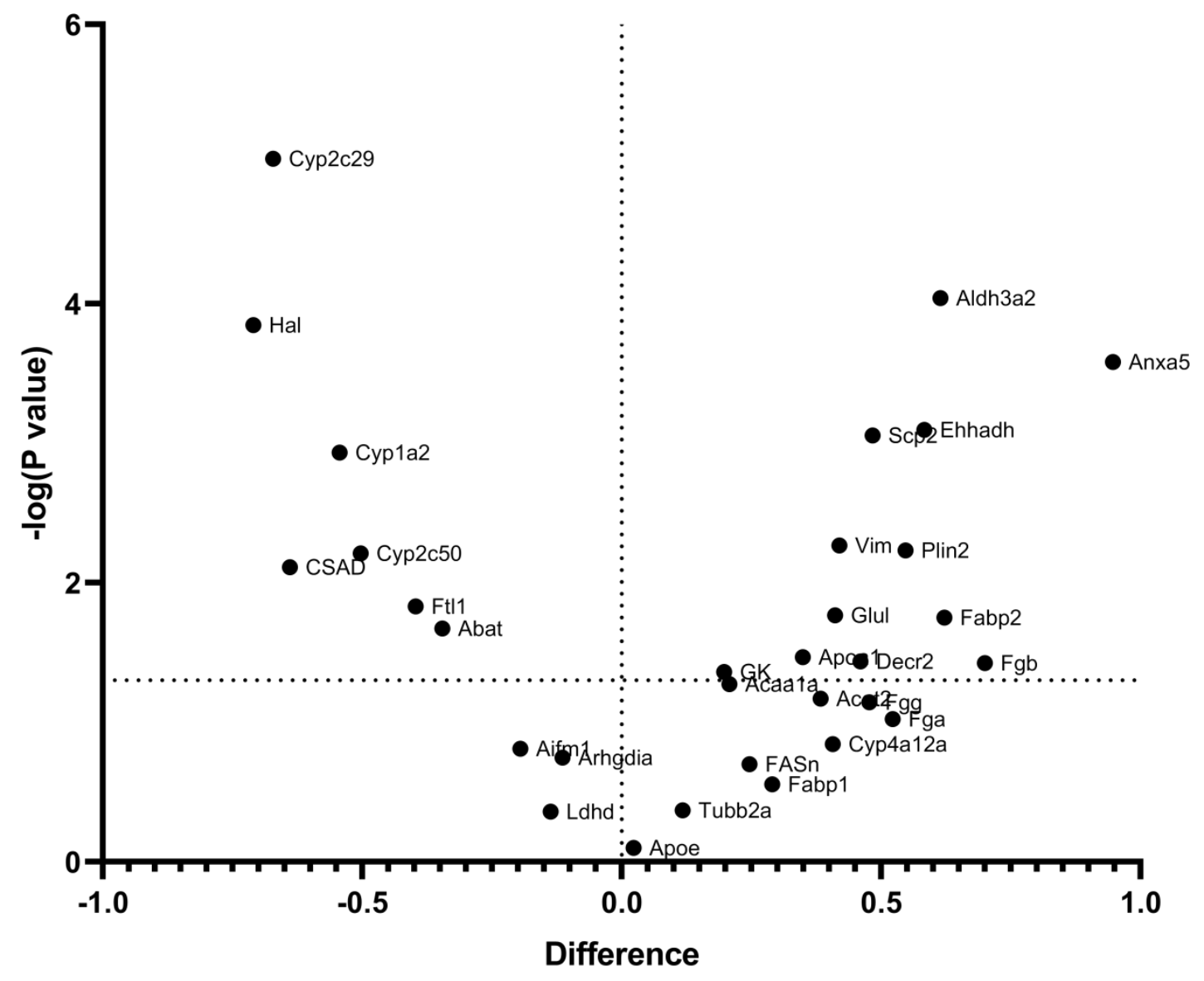

Figure 7.) Volcano Plot of High Fat Diet and Low Fat Diet Liver Proteins. Statistical significance was calculated using multiple t-tests for comparisons between each treatment groupwhere $p<$ $0.05^{*}, \mathrm{p}<0.01^{* *}, \mathrm{p}<0.001^{* *}, \mathrm{p}<0.0001^{* * * *}$, or not statistically significant (n.s). 


\section{Volcano Plot (HFD vs HMSX 0.5\%)}

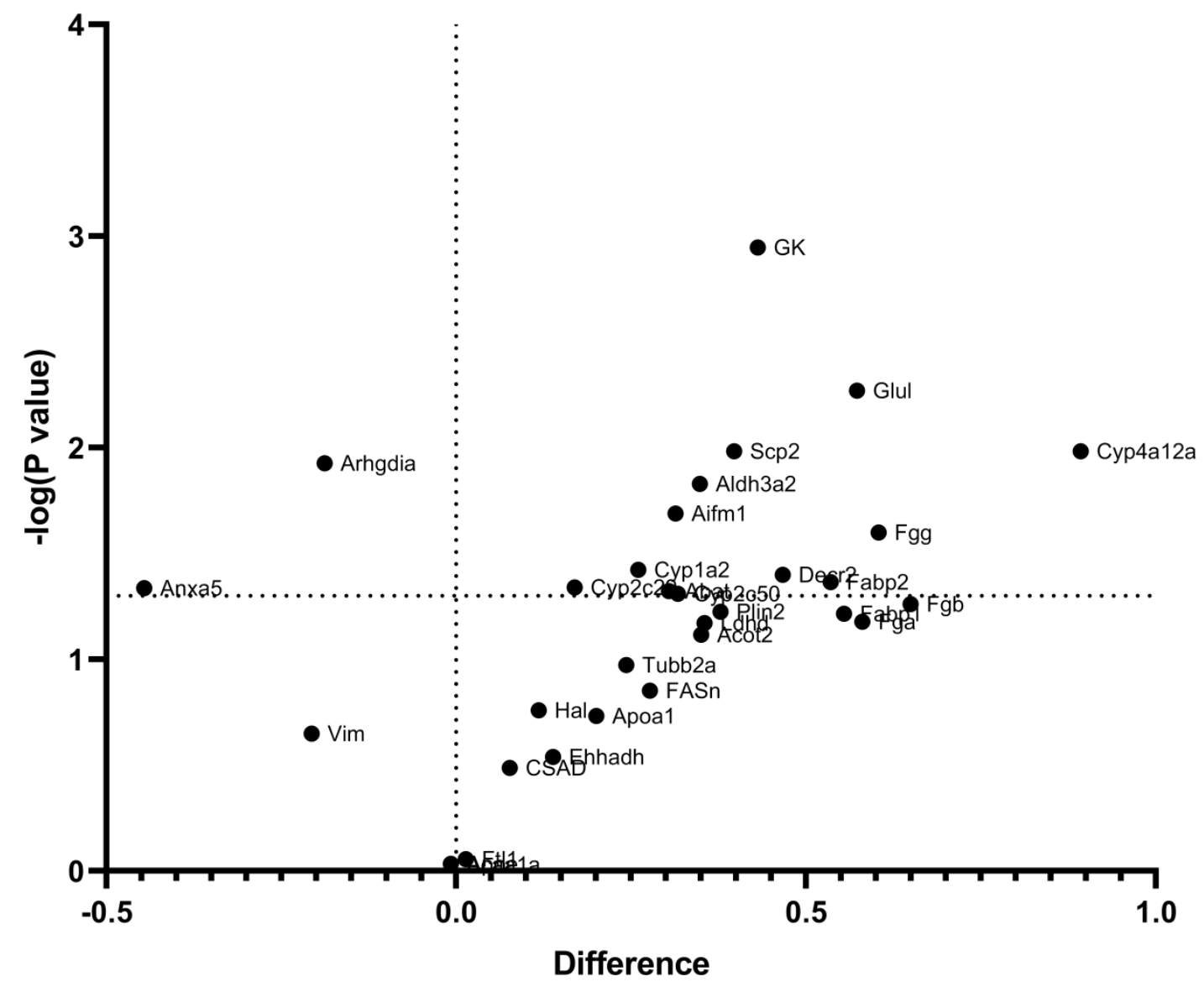

Figure 8.) Volcano Plot of High Fat Diet and High Fat Diet with MSX $0.5 \% \mathrm{w} / \mathrm{w}$ Liver Proteins Statistical significance was calculated using multiple t-tests for comparisons between each treatment groupwhere $\mathrm{p}<0.05^{*}, \mathrm{p}<0.01^{* *}, \mathrm{p}<0.001^{* * *}, \mathrm{p}<0.0001^{* * * *}$, or not statistically significant (n.s). 


\section{Volcano Plot (HFD vs HMSX0.05\%)}

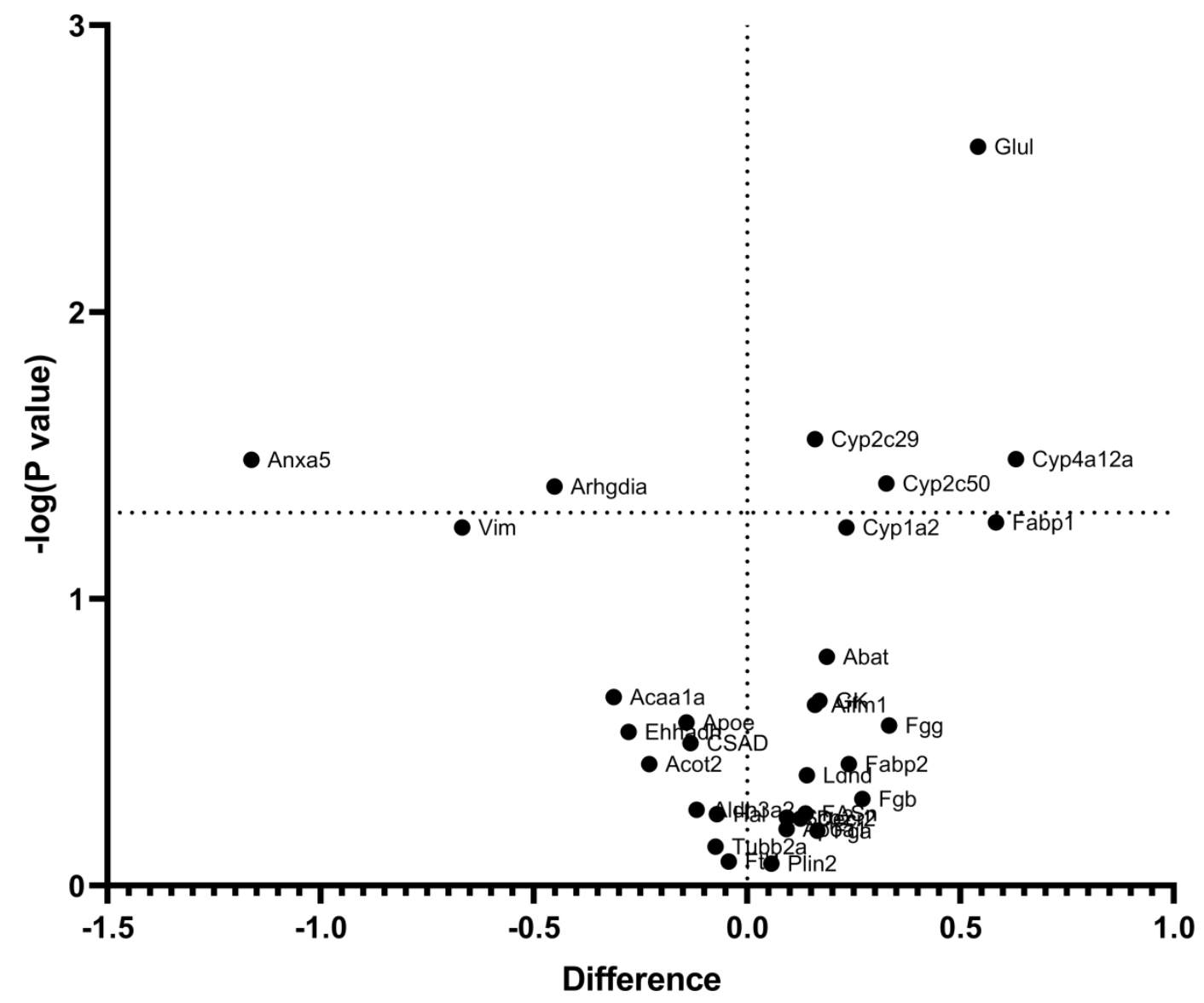

Figure 9.) Volcano Plot of High Fat Diet and High Fat Diet with MSX 0.05\% w/w Liver proteins.

Statistical significance was calculated using multiple t-tests for comparisons between each treatment groupwhere $\mathrm{p}<0.05^{*}, \mathrm{p}<0.01 * *, \mathrm{p}<0.001^{* * *}, \mathrm{p}<0.0001^{* * * *}$, or not statistically significant (n.s). 


\section{Volcano Plot (LFD vs. LMSX0.5\%)}

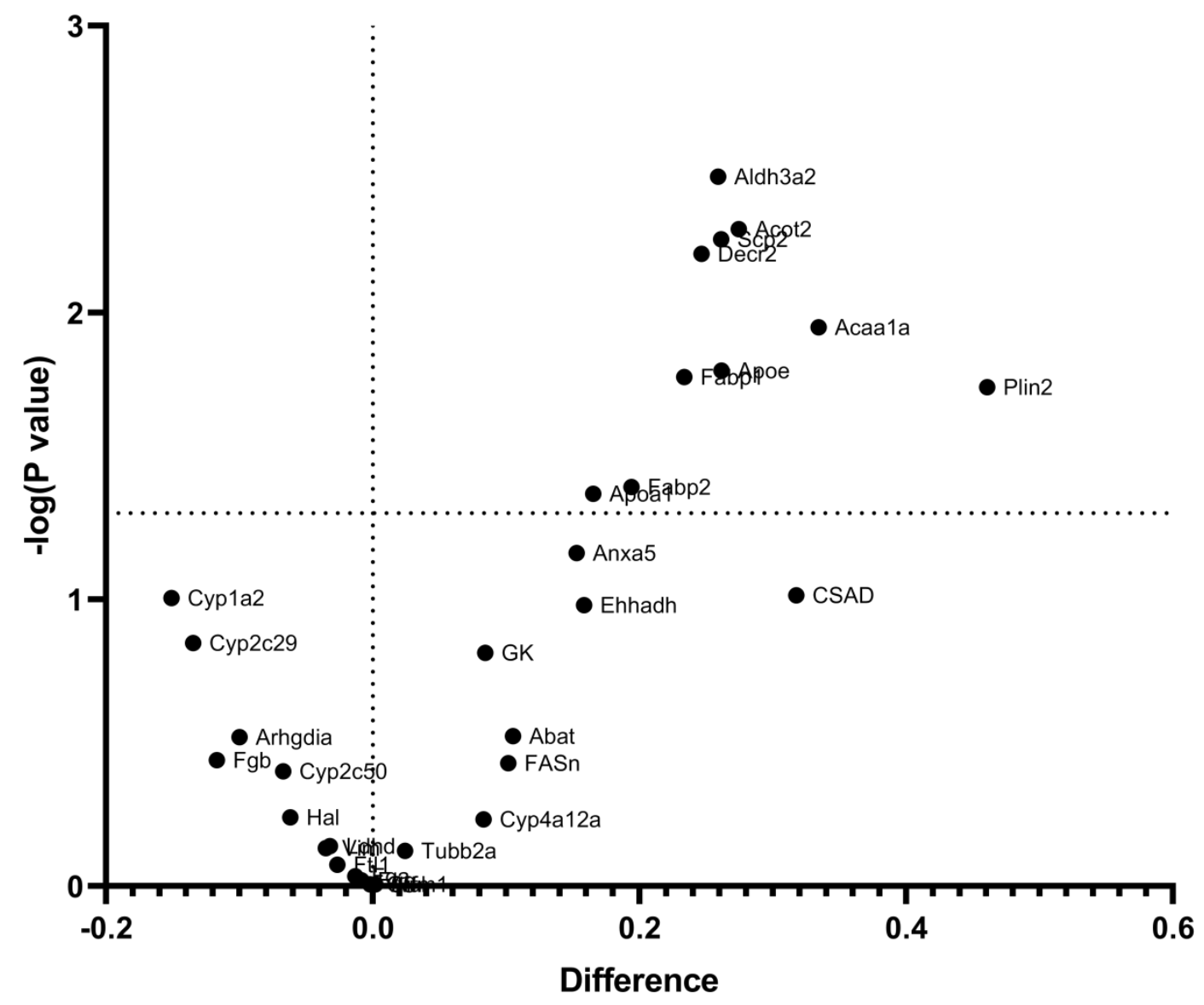

Figure 10) Volcano Plot of Low Fat Diet and Low Fat Diet with MSX 0.5\% w/w for Liver

Proteins. Statistical significance was calculated using multiple t-tests for comparisons between each treatment groupwhere $\mathrm{p}<0.05^{*}, \mathrm{p}<0.01^{* *}, \mathrm{p}<0.001^{* * *}, \mathrm{p}<0.0001^{* * * *}$, or not statistically significant (n.s). 


\section{Volcano Plot (LFD vs LMSX0.05\%)}

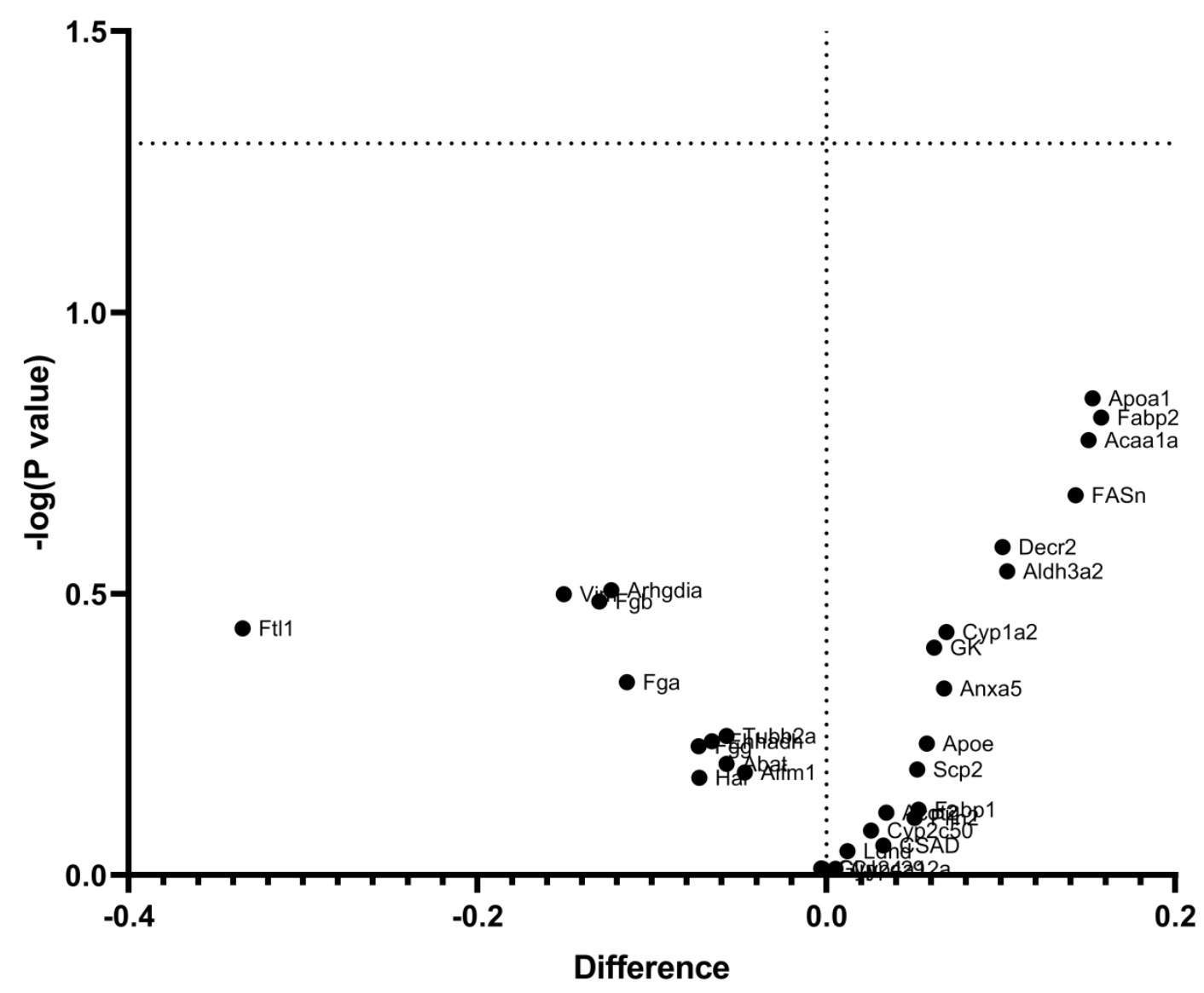

Figure 11.) Volcano Plot of Low Fat Diet and Low Fat Diet with MSX 0.05\% Liver Proteins. Statistical significance was calculated using multiple t-tests for comparisons between each treatment groupwhere $\mathrm{p}<0.05^{*}, \mathrm{p}<0.01 * *, \mathrm{p}<0.001^{* * *}, \mathrm{p}<0.0001^{* * * *}$, or not statistically significant (n.s). 
A

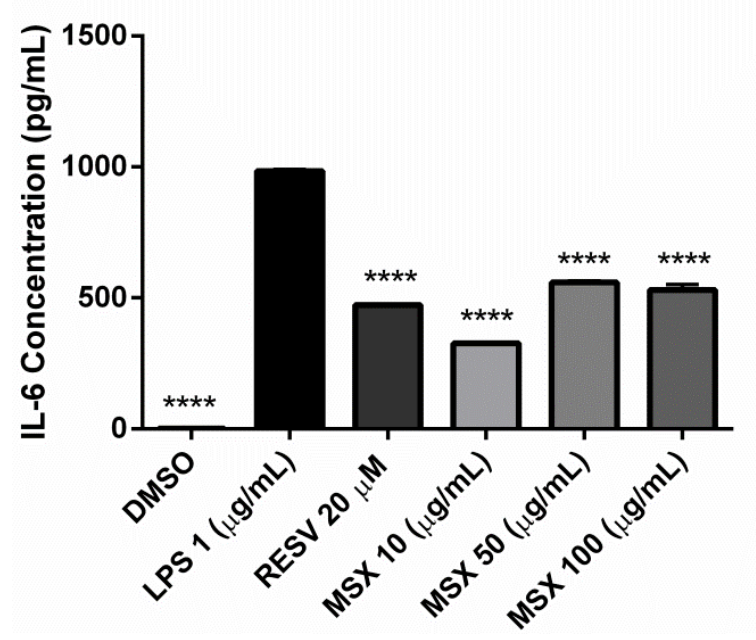

B

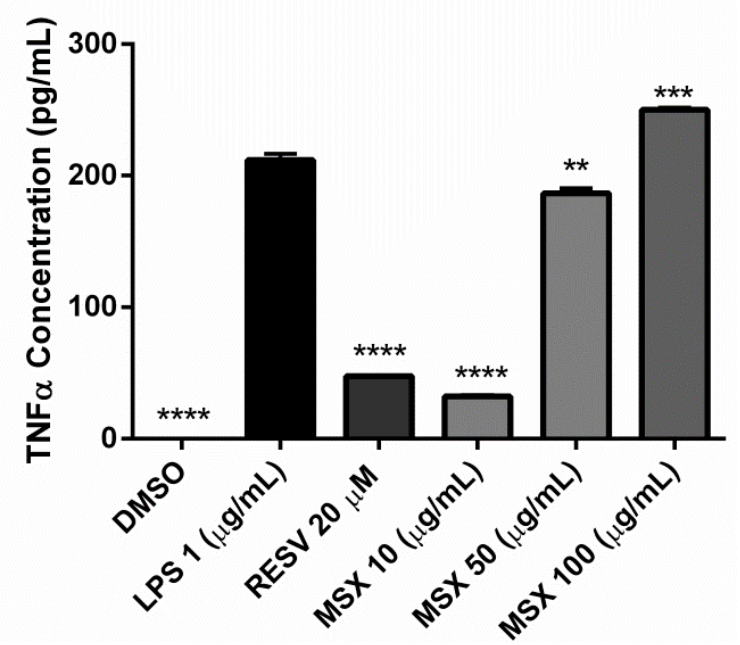

Figure 12) MSX mitigates initial LPS-induced inflammatory cytokine release in healthy human donor monocytes after $\mathbf{2 4}$ hours. Healthy Human Donor Monocytes were pre-treated with vehicle (DMSO) , RESV (20 $\mu \mathrm{M})$, or MSX at 10, 50 or $100 \mu \mathrm{g} / \mathrm{mL}$ for 1 hour before being exposed to LPS $(1 \mu \mathrm{g} / \mathrm{mL})$ for 24 hours. Media was then collected and saved for cytokine analysis using respective ELISAs. Data shown as mean \pm SE. All comparisons made to LPS $p<0.05^{*}, p<0.01$ $* *, p<0.001 * * *, p<0.0001 * * * *$. 
A

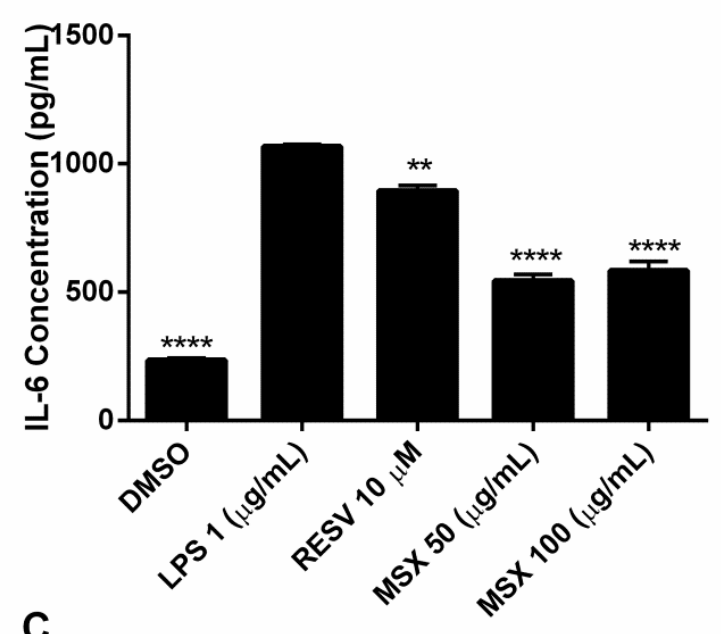

C

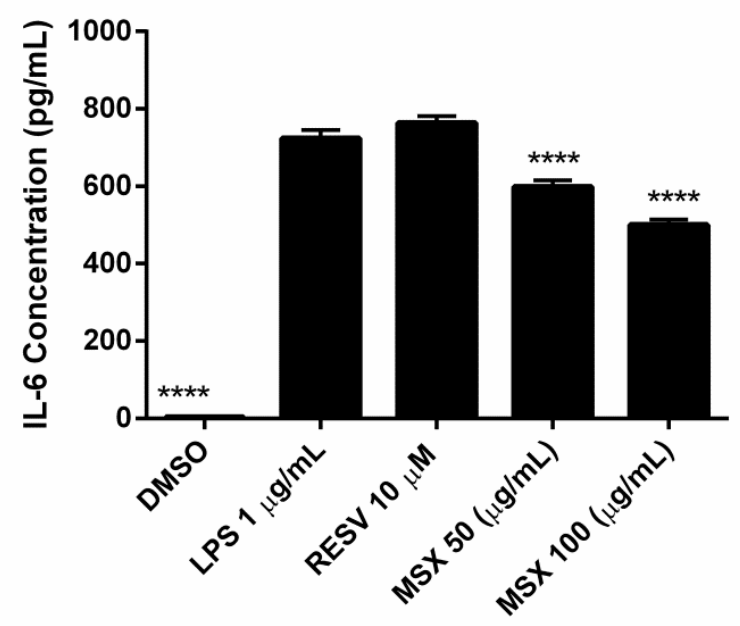

B
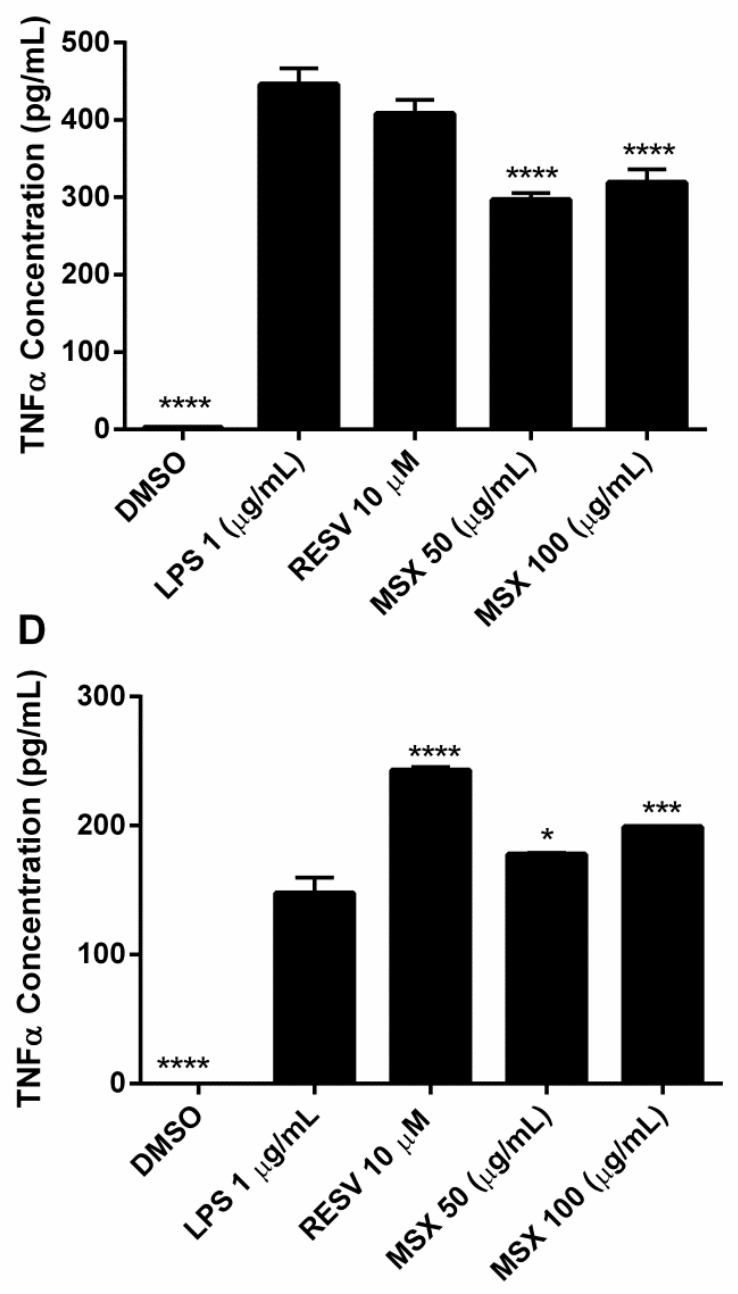

Figure 13.) MSX abrogates LPS-induced inflammatory cytokine release in differentiated THP-1 monocytes after 6 hrs. Differentiated THP-1 Cells were pre-treated with vehicle (DMSO), RESV $(10 \mu \mathrm{M})$, or MSX ( 50 or $100 \mu \mathrm{g} / \mathrm{mL}$ ) for 1 hour before being exposed to LPS $(1 \mu \mathrm{g} / \mathrm{mL}$ ) for 6 (Panels A and B) or 24 hours (Panels $C$ and D). Media was then collected and saved for cytokine analysis with respective ELISAs. Data shown as mean \pm SE. All comparisons made to LPS $p<$ $0.05^{*}, p<0.01^{* *}, p<0.001 * * *, p<0.0001 * * * *$. 

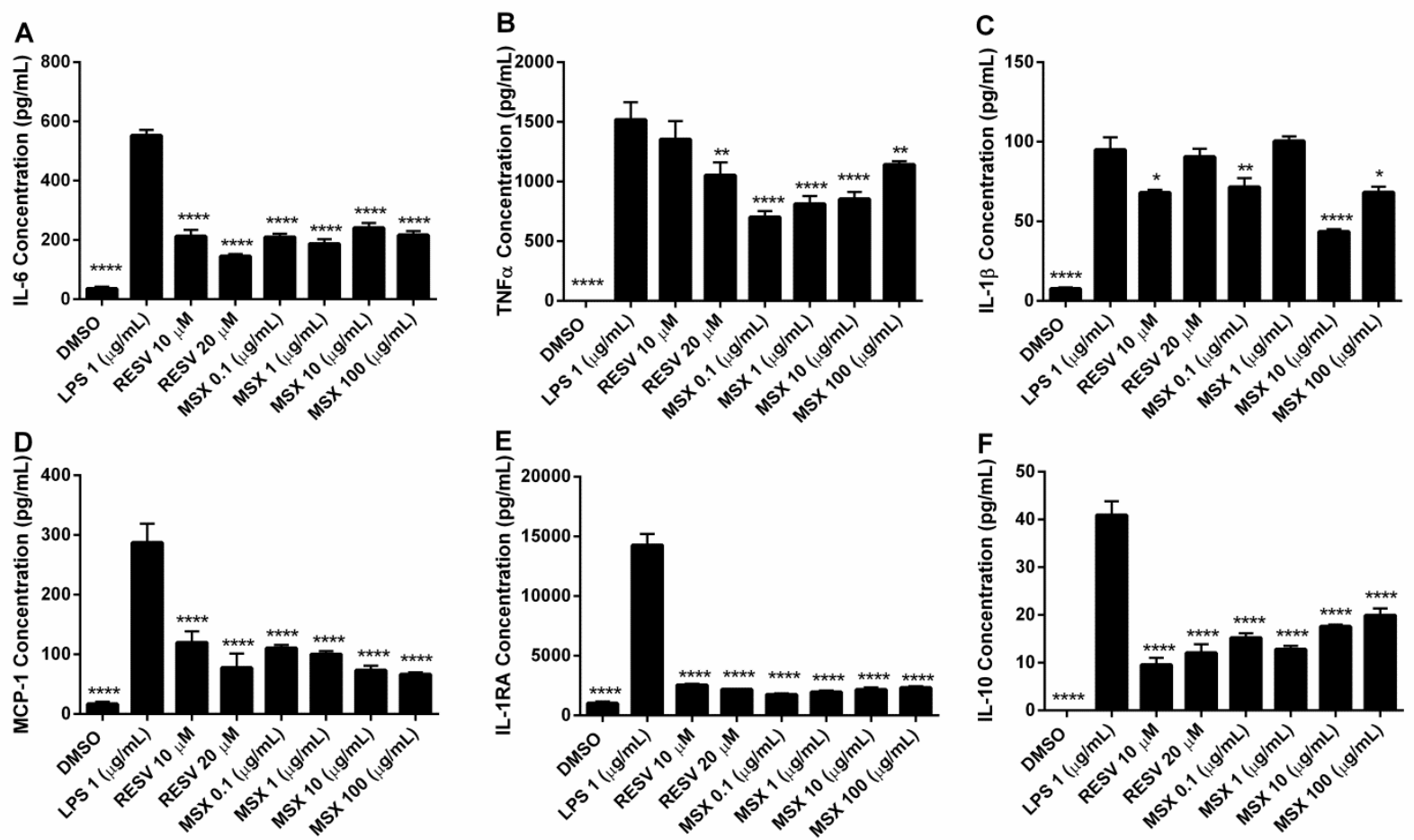

Figure 14.) Low doses of MSX abrogates LPS-induced inflammatory cytokine release in differentiated THP-1 monocytes after 6 hrs. Differentiated THP-1 Cells were pre-treated with vehicle (DMSO), RESV (20 $\mu \mathrm{M})$, or MSX $(0.1,1,1050$ or $100 \mu \mathrm{g} / \mathrm{mL})$ for 1 hour before being exposed to LPS $(1 \mu \mathrm{g} / \mathrm{mL})$ for 6 hours. Media was then collected and saved for cytokine analysis with a custom Procarta Cytokine Multiplex and analyzed using the Biorad Bioplex 200. Data shown as mean $\pm \mathrm{SE}$. All comparisons made to LPS $p<0.05^{*}, p<0.01^{* *}, p<0.001^{* * *}$, $p<0.0001 * * * *$. 

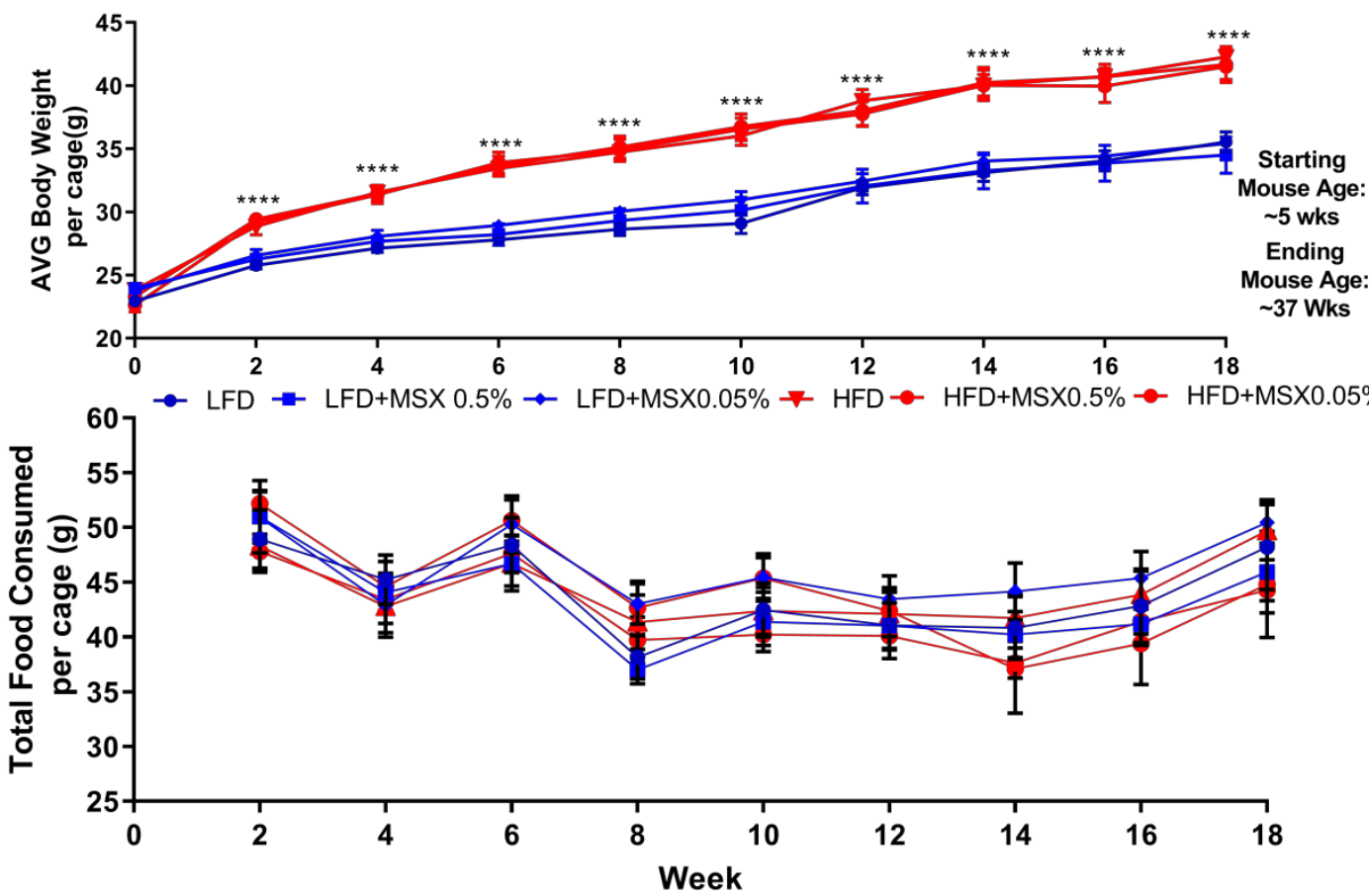

Supplemental Figure 1.) Average Body Weights and Food Intake of Each Cage for $\mathbf{1 8}$ Weeks of Diet Administration. Effects of each diet with and without $0.5 \%$ and $0.05 \% \mathrm{w} / \mathrm{w}$ MSX inclusion within either Low Fat or High Fat diet types. Data shown as mean $\pm \mathrm{SE}$. All comparisons made to LFD group where $\mathrm{p}<0.05^{*}, \mathrm{p}<0.01 * *, \mathrm{p}<0.001 * * *, \mathrm{p}<0.0001 * * * *$ 


\section{References and Works Cited}

1. Wolowczuk, I. Obesity - an inflammatory state. Acta Vet. Scand. 57, K5 (2015).

2. Wright, C. \& Simone, N. L. Obesity and tumor growth: inflammation, immunity, and the role of a ketogenic diet. Curr. Opin. Clin. Nutr. Metab. Care 19, 294 (2016).

3. Polyzos, S. A., Kountouras, J. \& Mantzoros, C. S. Obesity and nonalcoholic fatty liver disease: From pathophysiology to therapeutics. Metabolism (2018).

doi:10.1016/j.metabol.2018.11.014

4. Fändriks, L. Roles of the gut in the metabolic syndrome: an overview. J. Intern. Med. 281, 319-336 (2017).

5. Samson, S. L. \& Garber, A. J. Metabolic syndrome. Endocrinol. Metab. Clin. North Am. 43, 123 (2014).

6. Lumeng, C. N., Deyoung, S. M., Bodzin, J. L. \& Saltiel, A. R. Increased inflammatory properties of adipose tissue macrophages recruited during diet-induced obesity. Diabetes 56, 16-23 (2007).

7. Boutagy, N. E., McMillan, R. P., Frisard, M. I. \& Hulver, M. W. Metabolic endotoxemia with obesity: Is it real and is it relevant? Biochimie 124, 11-20 (2016).

8. Volynets, V. et al. Intestinal Barrier Function and the Gut Microbiome Are Differentially Affected in Mice Fed a Western-Style Diet or Drinking Water Supplemented with Fructose. J. Nutr. 147, 770-780 (2017).

9. Ferrere, G. et al. Activation of Kupffer Cells Is Associated with a Specific Dysbiosis Induced by Fructose or High Fat Diet in Mice. PLOS ONE 11, e0146177 (2016).

10. Eksteen, B. The Gut-Liver Axis in Primary Sclerosing Cholangitis. Clin. Liver Dis. 20, 1-14 (2016).

11. Kostic, A. D., Chun, E., Meyerson, M. \& Garrett, W. S. Microbes and inflammation in colorectal cancer. Cancer Immunol. Res. 1, 150-157 (2013). 
12. Dalmasso, G., Cougnoux, A., Delmas, J., Darfeuille-Michaud, A. \& Bonnet, R. The bacterial genotoxin colibactin promotes colon tumor growth by modifying the tumor microenvironment. Gut Microbes 5, 675-680 (2014).

13. Sartor, R. B. Microbial and Dietary Factors in the Pathogenesis of Chronic, Immune-Mediated Intestinal Inflammation. in Immune Mechanisms in Inflammatory Bowel Disease 35-54 (Springer, New York, NY, 2006). doi:10.1007/0-387-33778-4_4

14. Xu, H. et al. Chronic inflammation in fat plays a crucial role in the development of obesityrelated insulin resistance. J. Clin. Invest. 112, 1821-1830 (2003).

15. Berra, K. Treatment options for patients with the metabolic syndrome. J. Am. Acad. Nurse Pract. 15, 361-370 (2003).

16. Wagh, A. \& Stone, N. J. Treatment of metabolic syndrome. Expert Rev. Cardiovasc. Ther. 2, 213-228 (2004).

17. Baspinar, B., Eskici, G. \& Ozcelik, A. O. How coffee affects metabolic syndrome and its components. Food Funct. 8, 2089-2101 (2017).

18. Kojadinovic, M. I. et al. Consumption of Pomegranate juice decreases blood lipid peroxidation and levels of arachidonic acid in women with metabolic syndrome. J. Sci. Food Agric. 97, 1798-1804 (2017).

19. Saibandith, B., Spencer, J. P. E., Rowland, I. R. \& Commane, D. M. Olive Polyphenols and the Metabolic Syndrome. Mol. Basel Switz. 22, (2017).

20. Anhê, F. F. et al. Gut Microbiota Dysbiosis in Obesity-Linked Metabolic Diseases and Prebiotic Potential of Polyphenol-Rich Extracts. Curr. Obes. Rep. 4, 389-400 (2015).

21. Anhê, F. F. et al. A polyphenol-rich cranberry extract protects from diet-induced obesity, insulin resistance and intestinal inflammation in association with increased Akkermansia spp. population in the gut microbiota of mice. Gut 64, 872-883 (2015).

22. Perkins, T. D. \& van den Berg, A. K. Maple syrup-production, composition, chemistry, and sensory characteristics. Adv. Food Nutr. Res. 56, 101-143 (2009). 
23. Li, L. \& Seeram, N. P. Maple syrup phytochemicals include lignans, coumarins, a stilbene, and other previously unreported antioxidant phenolic compounds. J. Agric. Food Chem. 58, 11673-11679 (2010).

24. Zhang, Y. et al. Chemical compositional, biological, and safety studies of a novel maple syrup derived extract for nutraceutical applications. J. Agric. Food Chem. 62, 6687-6698 (2014).

25. Ma, H. et al. Effects of a Standardized Phenolic-Enriched Maple Syrup Extract on $\beta$-Amyloid Aggregation, Neuroinflammation in Microglial and Neuronal Cells, and $\beta$-Amyloid Induced Neurotoxicity in Caenorhabditis elegans. Neurochem. Res. 41, 2836-2847 (2016).

26. Miyoshi, J. et al. Minimizing confounders and increasing data quality in murine models for studies of the gut microbiome. PeerJ 6, e5166 (2018).

27. Tetri, L. H., Basaranoglu, M., Brunt, E. M., Yerian, L. M. \& Neuschwander-Tetri, B. A. Severe NAFLD with hepatic necroinflammatory changes in mice fed trans fats and a high-fructose corn syrup equivalent. Am. J. Physiol. - Gastrointest. Liver Physiol. 295, G987-G995 (2008).

28. Ito, M. et al. Longitudinal analysis of murine steatohepatitis model induced by chronic exposure to high-fat diet. Hepatol. Res. 37, 50-57 (2007).

29. Kleiner, D. E. et al. Design and validation of a histological scoring system for nonalcoholic fatty liver disease. Hepatol. Baltim. Md 41, 1313-1321 (2005).

30. van der Heijden, R. A. et al. High-fat diet induced obesity primes inflammation in adipose tissue prior to liver in C57BL/6j mice. Aging 7, 256-267 (2015).

31. Lund, M. E., To, J., O'Brien, B. A. \& Donnelly, S. The choice of phorbol 12-myristate 13acetate differentiation protocol influences the response of THP-1 macrophages to a proinflammatory stimulus. J. Immunol. Methods 430, 64-70 (2016).

32. Jamwal, R. et al. Multiplex and Label-Free Relative Quantification Approach for Studying Protein Abundance of Drug Metabolizing Enzymes in Human Liver Microsomes Using SWATHMS. J. Proteome Res. 16, 4134-4143 (2017). 
33. Prasad, B. et al. Interindividual variability in hepatic organic anion-transporting polypeptides and P-glycoprotein $(A B C B 1)$ protein expression: quantification by liquid chromatography tandem mass spectroscopy and influence of genotype, age, and sex. Drug Metab. Dispos. Biol. Fate Chem. 42, 78-88 (2014).

34. Wiśniewski, J. R. \& Rakus, D. Multi-enzyme digestion FASP and the 'Total Protein Approach'based absolute quantification of the Escherichia coli proteome. J. Proteomics 109, 322-331 (2014).

35. Cox, J. \& Mann, M. MaxQuant enables high peptide identification rates, individualized p.p.b.range mass accuracies and proteome-wide protein quantification. Nat. Biotechnol. 26, 13671372 (2008).

36. Yang, Y., Smith, D. L., Keating, K. D., Allison, D. B. \& Nagy, T. R. Variations in body weight, food intake and body composition after long-term high-fat diet feeding in C57BL/6J Mice. Obes. Silver Spring Md 22, 2147-2155 (2014).

37. Blackwell, B.-N., Bucci, T. J., Hart, R. W. \& Turturro, A. Longevity, Body Weight, and Neoplasia in Ad Libitum-Fed and Diet-Restricted C57BL6 Mice Fed NIH-31 Open Formula Diet. Toxicol. Pathol. 23, 570-582 (1995).

38. Dakin, R. S., Walker, B. R., Seckl, J. R., Hadoke, P. W. F. \& Drake, A. J. Estrogens protect male mice from obesity complications and influence glucocorticoid metabolism. Int. J. Obes. 2005 39, 1539-1547 (2015).

39. Riant, E. et al. Estrogens protect against high-fat diet-induced insulin resistance and glucose intolerance in mice. Endocrinology 150, 2109-2117 (2009).

40. Ballestri, S. et al. NAFLD as a Sexual Dimorphic Disease: Role of Gender and Reproductive Status in the Development and Progression of Nonalcoholic Fatty Liver Disease and Inherent Cardiovascular Risk. Adv. Ther. 34, 1291-1326 (2017).

41. Xin, G. et al. Sex hormone affects the severity of non-alcoholic steatohepatitis through the MyD88-dependent IL-6 signaling pathway. Exp. Biol. Med. Maywood NJ 240, 1279-1286 (2015).

42. National Center for Health Statistics (US). Health, United States, 2011: With Special Feature on Socioeconomic Status and Health. (National Center for Health Statistics (US), 2012). 
43.WWEIA Data Tables : USDA ARS. Available at: https://www.ars.usda.gov/northeastarea/beltsville-md-bhnrc/beltsville-human-nutrition-research-center/food-surveys-researchgroup/docs/wweia-data-tables/. (Accessed: 18th February 2019)

44.Savard, C. et al. Synergistic Interaction of Dietary Cholesterol and Dietary Fat in Inducing Experimental Steatohepatitis. Hepatol. Baltim. Md 57, 81-92 (2013).

45.Panasevich, M. R. et al. High-fat, high-fructose, high-cholesterol feeding causes severe NASH and cecal microbiota dysbiosis in juvenile Ossabaw swine. Am. J. Physiol. Endocrinol. Metab. 314, E78-E92 (2018).

46. Rinella, M. E. et al. Mechanisms of hepatic steatosis in mice fed a lipogenic methionine choline-deficient diet. J. Lipid Res. 49, 1068-1076 (2008).

47.Mells, J. E. et al. Saturated fat and cholesterol are critical to inducing murine metabolic syndrome with robust nonalcoholic steatohepatitis. J. Nutr. Biochem. 26, 285-292 (2015).

48.Kakehashi, A. et al. Proteome Characteristics of Non-Alcoholic Steatohepatitis Liver Tissue and Associated Hepatocellular Carcinomas. Int. J. Mol. Sci. 18, (2017).

49.Alzaid, F. et al. IRF5 governs liver macrophage activation that promotes hepatic fibrosis in mice and humans. JCI Insight 1, (2016).

50.Csak, T. et al. microRNA-122 regulates hypoxia-inducible factor-1 and vimentin in hepatocytes and correlates with fibrosis in diet-induced steatohepatitis. Liver Int. Off. J. Int. Assoc. Study Liver 35, 532-541 (2015).

51.Basaranoglu, M., Basaranoglu, G. \& Bugianesi, E. Carbohydrate intake and nonalcoholic fatty liver disease: fructose as a weapon of mass destruction. Hepatobiliary Surg. Nutr. 4, 109-116 (2015).

52. Miinalainen, I. J. et al. Mitochondrial 2,4-dienoyl-CoA reductase deficiency in mice results in severe hypoglycemia with stress intolerance and unimpaired ketogenesis. PLoS Genet. 5, e1000543 (2009).

53. Houten, S. M. et al. Peroxisomal L-bifunctional enzyme (Ehhadh) is essential for the production of medium-chain dicarboxylic acids. J. Lipid Res. 53, 1296-1303 (2012). 
54.Leroux, A. et al. Toxic lipids stored by Kupffer cells correlates with their pro-inflammatory phenotype at an early stage of steatohepatitis. J. Hepatol. 57, 141-149 (2012).

55.Atshaves, B. P. et al. Overexpression of sterol carrier protein-2 differentially alters hepatic cholesterol accumulation in cholesterol-fed mice. J. Lipid Res. 50, 1429-1447 (2009).

56.Liang, L. et al. Loss of ARHGDIA expression is associated with poor prognosis in HCC and promotes invasion and metastasis of HCC cells. Int. J. Oncol. 45, 659-666 (2014).

57.Libby, A. E., Bales, E., Orlicky, D. J. \& McManaman, J. L. Perilipin-2 Deletion Impairs Hepatic Lipid Accumulation by Interfering with Sterol Regulatory Element-binding Protein (SREBP) Activation and Altering the Hepatic Lipidome. J. Biol. Chem. 291, 24231-24246 (2016).

58.Palmieri, E. M. et al. Glutamine synthetase desensitizes differentiated adipocytes to proinflammatory stimuli by raising intracellular glutamine levels. FEBS Lett. 588, 4807-4814 (2014).

59.Greaves, P. et al. Uroporphyria and hepatic carcinogenesis induced by polychlorinated biphenyls-iron interaction: absence in the Cyp1a2(-/-) knockout mouse. Biochem. Biophys. Res. Commun. 331, 147-152 (2005).

60.Capdevila, J. H., Falck, J. R. \& Harris, R. C. Cytochrome P450 and arachidonic acid bioactivation. Molecular and functional properties of the arachidonate monooxygenase. J. Lipid Res. 41, 163-181 (2000).

61.Wang, H. et al. Cloning, expression, and characterization of three new mouse cytochrome p450 enzymes and partial characterization of their fatty acid oxidation activities. Mol. Pharmacol. 65, 1148-1158 (2004).

62.Jeffery, B. et al. Peroxisome proliferator activated receptor alpha regulates a male-specific cytochrome P450 in mouse liver. Arch. Biochem. Biophys. 429, 231-236 (2004).

63.Lombardi, R., Pisano, G. \& Fargion, S. Role of Serum Uric Acid and Ferritin in the Development and Progression of NAFLD. Int. J. Mol. Sci. 17, 548 (2016).

64.Sandoval-Salazar, C., Ramírez-Emiliano, J., Trejo-Bahena, A., Oviedo-Solís, C. I. \& Solís-Ortiz, M. S. A high-fat diet decreases GABA concentration in the frontal cortex and hippocampus of rats. Biol. Res. 49, 15 (2016). 
65.Sohrabipour, S., Sharifi, M. R., Talebi, A., Sharifi, M. \& Soltani, N. GABA dramatically improves glucose tolerance in streptozotocin-induced diabetic rats fed with high-fat diet. Eur. J. Pharmacol. 826, 75-84 (2018).

66.Kelley, G. L. \& Azhar, S. Reversal of high dietary fructose-induced PPARalpha suppression by oral administration of lipoxygenase/cyclooxygenase inhibitors. Nutr. Metab. 2, 18 (2005).

67.Kelley, G. L., Allan, G. \& Azhar, S. High dietary fructose induces a hepatic stress response resulting in cholesterol and lipid dysregulation. Endocrinology 145, 548-555 (2004).

68. Roncaglia, L., Amaretti, A., Raimondi, S., Leonardi, A. \& Rossi, M. Role of bifidobacteria in the activation of the lignan secoisolariciresinol diglucoside. Appl. Microbiol. Biotechnol. 92, 159168 (2011).

69.Tominaga, S. et al. (-)-Secoisolariciresinol attenuates high-fat diet-induced obesity in C57BL/6 mice. Food Funct. 3, 76-82 (2012).

70.Drygalski, K. et al. Does the enterolactone (ENL) affect fatty acid transporters and lipid metabolism in liver? Nutr. Metab. 14, 69 (2017).

71.Berk, K. et al. The effect of enterolactone on liver lipid precursors of inflammation. Life Sci. 221, 341-347 (2019).

72.Bosshart, H. \& Heinzelmann, M. THP-1 cells as a model for human monocytes. Ann. Transl. Med. 4, (2016).

73.Chanput, W., Mes, J., Vreeburg, R. A. M., Savelkoul, H. F. J. \& Wichers, H. J. Transcription profiles of LPS-stimulated THP-1 monocytes and macrophages: a tool to study inflammation modulating effects of food-derived compounds. Food Funct. 1, 254-261 (2010).

74.Moors, M. A. \& Mizel, S. B. Proteasome-mediated regulation of interleukin-1beta turnover and export in human monocytes. J. Leukoc. Biol. 68, 131-136 (2000).

75.Latz, E., Xiao, T. S. \& Stutz, A. Activation and regulation of the inflammasomes. Nat. Rev. Immunol. 13, (2013).

76.Tan, Q. et al. The Role of IL-1 Family Members and Kupffer Cells in Liver Regeneration. BioMed Res. Int. 2016, (2016). 


\section{MANUSCRIPT 4}

Published Nutritional Neuroscience Aug 2017 doi: 10.1080/1028415X.2017.1360558

\section{Pomegranate ellagitannin-gut microbial derived metabolites, urolithins, inhibit neuroinflammation in vitro}

Nicholas A. DaSilva ${ }^{1}$, Pragati P. Nahar ${ }^{1}$, Hang Ma $^{1}$, Aseel Eid ${ }^{1}$, Zhengxi Wei ${ }^{1}$, Susan Meschwitz ${ }^{2}$, Nasser H. Zawia ${ }^{1,3}$, Angela L. Slitt ${ }^{1}$, Navindra P. Seeram ${ }^{1,3}$

${ }^{1}$ Department of Biomedical and Pharmaceutical Sciences, College of Pharmacy, University of Rhode Island Kingston, RI 02881 USA

${ }^{2}$ Department of Chemistry, Salve Regina University, Newport, RI 02840 USA

${ }^{3}$ George and Anne Ryan Institute for Neuroscience, University of Rhode Island, Kingston RI 02881 USA

Corresponding Authors:

Nasser H. Zawia; University of Rhode Island, 395F, Avedisian Hall, 7 Greenhouse Rd. Kingston, RI 02881, USA, Phone: 401-874-5368, Email: nzawia@uri.edu

Angela L. Slitt; University of Rhode Island, 395D, Avedisian Hall, 7 Greenhouse Rd. Kingston, RI 02881, USA, Phone: 401-874-5020, Email: aslitt@uri.edu

Navindra P. Seeram; University of Rhode Island , 495B, Avedisian Hall, 7 Greenhouse Rd.

Kingston, RI, 02881, USA, Phone: 401-874-9367, Email: nseeram@uri.edu 


\section{Abstract}

Objectives: Urolithins, ellagitannin-gut microbial derived metabolites, have been reported to mediate Pomegranate's neuroprotective effects against Alzheimer's disease (AD) but there is limited data on their effects against neuroinflammation. Herein, we: 1) evaluated whether urolithins (urolithins $A$ and $B$ and their methylated derivatives) attenuate neuroinflammation in murine BV-2 microglia and human SH-SY5Y neurons, and 2) evaluated hippocampus of transgenic AD (R1.40) mice administered a Pomegranate extract (PE; 100 or $200 \mathrm{mg} / \mathrm{kg} / \mathrm{day}$ for 3 weeks) for inflammatory biomarkers.

Methods: Effects of urolithins $(10 \mu \mathrm{M})$ on inflammatory biomarkers were evaluated in lipopolysaccharide (LPS)-stimulated BV-2 microglia. In a non-contact co-culture cell model, SHSY5Y cell viability was assessed after exposure to media collected from LPS-BV-2 cells treated with or without urolithins. Effects of urolithins on apoptosis and caspases $3 / 7$ and 9 release from $\mathrm{H}_{2} \mathrm{O}_{2}$-induced oxidative stress of BV-2 and SH-SY5Y cells were assessed. Hippocampal tissues of vehicle and PE-treated transgenic R1.40 mice were evaluated for gene expression of inflammatory biomarkers by qRT-PCR.

Results: Urolithins decreased media levels of NO, IL-6, PGE2, and TNF $\alpha$ from LPS-BV-2 microglia. In the co-culture cell model, media from urolithins-LPS-BV-2 treated cells preserved SH-SY5Y cell viability greater than media from non-urolithin treated cells. Urolithins mitigated apoptosis and caspases $3 / 7$ and 9 release from $\mathrm{H}_{2} \mathrm{O}_{2}$-induced oxidative stress of $\mathrm{BV}-2$ and $\mathrm{SH}-\mathrm{SY} 5 \mathrm{Y}$ cells. While not statistically significant, inflammatory biomarkers (TNF- $\alpha$, COX-2, IL-1, and IL-6) appeared to follow a decreasing trend in the hippocampus of high-dose PE-treated animals compared to controls.

Discussion: The attenuation of neuroinflammation by urolithins may contribute, in part, towards Pomegranate's neuroprotective effects against AD. 
Keywords: Pomegranate, Urolithins, Gut microflora, Neuroinflammation, Neuroprotective 


\section{Introduction}

Neurodegenerative diseases including Alzheimer's disease (AD), are associated with

neuroinflammation, which initiates the degeneration of neurons by over-activating microglia in the brain. ${ }^{1}$ Serving as the resident macrophages of the brain, microglia respond to oxidative stress derived from tissue damage (i.e. ischemic stroke or trauma) and pathogens to prevent

further damage to the fragile network of cells. ${ }^{2}$ Moreover, microglial activation, for instance, from bacterial derived lipopolysaccharide (LPS), results in the expression of inflammatory mediators and cytokines including nitric oxide (NO), interleukin 6 (IL-6), tumor necrosis factor alpha (TNFa), and prostaglandin $\mathrm{E}_{2}\left(\mathrm{PGE}_{2}\right)$. These inflammatory biomarkers induce a proinflammatory environment by initiating aberrant protein function/formation in the form of microtubule associated protein tau (tau) malignancies, $\beta$-amyloid, $\alpha$-synuclein, or Lewy body production, finally triggering apoptotic pathways (caspase activation). ${ }^{3}$ The aforementioned process of reactive microgliosis has been extensively studied and is implicated in AD pathogenesis. ${ }^{4}$ Moreover, inflammation of microglia by reactive species from other microglia, and exposure to tau, $\beta$-amyloid oligomers, or even LPS, activates microglia in a similar convergent pathway that ultimately requires the initiation of executioner proteins, caspase 3 and 7 , to continue with programmed cell death. Therefore, the prevention and/or modulation of key pro-inflammatory markers from microglia, achievable through dietary natural products and plant foods, are promising dietary strategies for the prevention and possible treatment of AD. 
The Pomegranate (Punica granatum L.) fruit, which is popularly consumed as juice and botanical dietary supplements/extracts, is a rich source of ellagitannins, a class of bioactive polyphenols, which release ellagic acid on hydrolysis. Extensive human and animal studies have shown that while Pomegranate ellagitannins are not bioavailable, their gut microbial-derived metabolites, known as urolithins (formed from colonic microflora biotransformation of ellagic acid), achieve detectable concentrations and persist through enterohepatic circulation in vivo. ${ }^{5}$ Consequently, urolithins are widely regarded as relevant in vivo compounds which contribute, in part, to the biological effects attributed to Pomegranate consumption. ${ }^{5}$

Published studies support the neuroprotective effects of Pomegranate juice and extracts against $A D$ pathogenesis in several transgenic $A D$ animal models. ${ }^{6-10}$ Our group has also reported on the neuroprotective effects of an ellagitannin-enriched Pomegranate extract $(\mathrm{PE})$ in a transgenic $A D$ animal (R1.40) model. ${ }^{11}$ We further reported that the neuroprotective effects of the PE were most likely mediated by urolithins given that its naturally occurring ellagitannins and other phytochemicals do not fulfill criteria (based on in silico computational data) required for blood brain barrier penetration. ${ }^{12}$ Urolithins have been shown to have anti-proliferative and anti-inflammatory effects in breast, colon, and neuroblastoma cells ${ }^{13-15}$ but there is limited data on their inhibitory effects against neuroinflammation in microglia and neuronal cells. 
Therefore, herein, we: 1) evaluated the in vitro inhibitory effects of urolithins (urolithins $A$ and $B$ and their methylated derivatives) against neuroinflammation in murine BV-2 microglia and differentiated human neuronal SH-SY5Y cell models and, 2) conducted secondary analysis of brain tissues (collected from our previously reported $A D$ animal study with $P E)^{11}$ for its in vivo inhibitory effects on inflammatory biomarkers.

\section{Materials and methods}

\section{Chemicals and General Procedures}

Dimethyl sulfoxide (DMSO) was obtained from Thermo Fisher Scientific (Boston, MA, USA).

Trans-resveratrol (RESV), all-trans retinoic acid (RA), trypan blue, hydrogen peroxide $\left(\mathrm{H}_{2} \mathrm{O}_{2}\right)$, and lipopolysaccharide (LPS) were obtained from Sigma Aldrich (St. Louis, MO, USA). Urolithins (6Hdibenzo[b,d]pyran-6-one derivatives), namely, urolithin A (3,8-dihydroxy-6H-dibenzo[b,d]pyran6-one; UA), methylated-urolithin A (3-hydroxy-8-methoxy-6H-dibenzo[b,d]pyran-6-one; $\mathrm{mUA}$ ), urolithin B (3-hydroxy-6H-dibenzo[b,d]pyran-6-one; UB), and methylated-urolithin B (3methoxy-6H-dibenzo[b,d]pyran-6-one; $m U B$ ) were synthesized and validated for purity in our laboratory according to previously reported methods. ${ }^{12}$ 


\section{Alzheimer's Disease Transgenic R1.40 Mouse Study}

The AD animal study was conducted as previously reported by our group using an aged transgenic R1.40 (B6.129-Tg(APPSw)40Btla/Mmjax) mouse model. ${ }^{11}$ Briefly, a standardized ellagitannin-enriched Pomegranate extract (POMELLA ${ }^{\circledR}$ ), obtained from Verdure Sciences (Noblesville, IN, USA), was administered to the mice by oral gavage at doses of 100 and 200 $\mathrm{mg} / \mathrm{kg}$ for three weeks. All experiments were approved by the University of Rhode Island Institutional Action Care and Use Committee and animals were kept under supervision by a veterinarian throughout the course of the study and during PE administration. On day 15 of PE treatment, the mice began the Morris water maze task, lasting for ten days, followed by a probe trial and on day 36 , the mice performed the $\mathrm{Y}$-maze task for a single day. The data accumulated from the behavioral tests have been previously reported. ${ }^{11}$ On day 37 , mice were euthanized and brain tissues were collected and stored at $-80^{\circ} \mathrm{C}$. While the majority of the brain tissues were utilized for molecular biology assays as previously reported, ${ }^{11}$ the limited remaining hippocampal tissues were used for the current study (further described below).

\section{RNA Extraction and qRT-PCR of Hippocampal Mouse Tissue for Inflammatory Markers}

For the gene expression studies, RNA was extracted from hippocampal brain tissues using the Trizol method as previously described. ${ }^{11}$ RNA samples were stored at $-80{ }^{\circ} \mathrm{C}$ until use. The RNA concentration was determined by measuring UV absorbance of the sample at $260 \mathrm{~nm}$ using a NanoDrop 1000 (Thermo Scientific, Wilmington, DE, USA). Total RNA (1 $\mu \mathrm{g})$ was reverse transcribed to cDNA using the iScript cDNA Synthesis Kit (Bio-Rad, Hercules, CA, USA). 
Gene expression was measured in qRT-PCR reactions using SYBR-green with gene-specific oligonucleotide primers (Life Technologies, Grand Island, NY, USA) using LightCycler 480 (Roche Applied Science, Indianapolis, IN, USA). Relative mRNA expression of each gene was normalized to the $\beta$-actin housekeeping gene. The experiment was performed in triplicate. The primers utilized were as follows:

COX2-F: TGGGGTGATGAGCAACTATT;

COX2-R: AAGGAGCTCTGGGTCAAACT;

IL-1ß-F: GACCTTCCAGGATGAGGACA;

IL-1ß-R: AGCTCATATGGGTCCGACAG;

IL-6-F: AGTTGCCTTCTTGGGACTGA ;

IL-6-R: CAGAATTGCCATTGCACAAC;

TNF $\alpha-F: \quad$ ACGGCATGGATCTCAAAGAC;

TNF $\alpha$-RGTGGGTGAGGAGCACGTAGT.

\section{Cell Culture}

Human neuroblastoma SH-SY5Y cells were obtained from the American Type Culture Collection (Manassas, VA, USA) and were differentiated into neuronal cells as previously reported by our group. ${ }^{16}$ Briefly, the SH-SY5Y cells were treated with retinoic acid (RA) (10 $\left.\mu \mathrm{M}\right)$ for 7 days while changing media every $48 \mathrm{~h}$ to ensure proper neurite growth and cell morphology. Murine microglia BV-2 cells were kindly provided by Professor Grace Sun from the University of Missouri at Columbia. 
The BV-2 and SH-SY5Y cell lines were maintained using high glucose (4.5 g/L) DMEM/F12 (Life Technologies, Grand Island, NY, USA) supplemented with 10\% heat inactivated FBS (Life Technologies, Grand Island, NY, USA), 1\% P/S (100 U/ml penicillin, $100 \mu \mathrm{g} / \mathrm{mL}$ streptomycin (Gibco/Life Technologies, Grand Island, NY, USA) and incubated in $5 \% \mathrm{CO}_{2}$ at $37^{\circ} \mathrm{C}$. The cells were harvested by trypsinization (0.25\% Trypsin/ EDTA, Life Technologies, Grand Island, NY, USA) and were then centrifuged (1500 rpm for $5 \mathrm{~min}$ ) and resuspended in 10\% DMEM/F12. Cell concentrations were determined by counting cells with a hemocytometer and viability was assessed by trypan blue staining. All experimental compounds (urolithins at $10 \mu \mathrm{M}$; RESV at 20 $\mu \mathrm{M}$ ) were dissolved in DMSO to yield a $10 \mathrm{mM}$ stock solution. Compounds were further diluted to yield solutions with a final DMSO concentration of less than $0.1 \%$. Trans-resveratrol (RESV) was used as a positive control to demonstrate the protective properties of a well-studied bloodbrain-barrier penetrable dietary polyphenol.

\section{Cell Viability and Oxidative Stress Assays}

Cellular viability was assessed using the Cell Titer Glo 2.0 (CTG 2.0) one step assay (Promega, Madison, WI, USA). Briefly, BV-2 and SH-SY5Y cells were seeded at 100,000 cells/mL to yield 80 $85 \%$ confluency in a standard white walled clear bottom 96 -well plate. BV-2 cells were exposed to experimental compounds (urolithins at $10 \mu \mathrm{M}$; RESV at $20 \mu \mathrm{M}$ ) and controls for $1 \mathrm{~h}$ prior to induction of oxidative stress with $100 \mu \mathrm{M}$ of $\mathrm{H}_{2} \mathrm{O}_{2}$ for $6 \mathrm{~h}$. SH-SY5Y cells were allowed to incubate with the compounds and controls for $24 \mathrm{~h}$ prior to exposure of $100 \mu \mathrm{M}$ of $\mathrm{H}_{2} \mathrm{O}_{2}$ for $6 \mathrm{~h}$. 
Following the pre-determined incubation period $(6,24$, or $48 \mathrm{~h})$, CTG 2.0 was added in a 1:1 ratio with existing media and mixed for 2 minutes on an orbital shaker prior to luminescence measurement (Spectramax M2, Molecular Devices, Sunnyvale, CA, USA).

\section{Non-Contact Co-Culture Cell Model}

BV-2 and SH-SY5Y cells were co-cultured as previously reported by our group. ${ }^{16}$ Briefly, SH-SY5Y cells were plated in 96 well plates at a density of 100,000 cells/mL and allowed to attach for $24 \mathrm{~h}$ following seeding. After confirming attachment and a confluency of $85 \%$, SH-SY5Y cells were differentiated for 7 days using RA (10 $\mu \mathrm{M})$ as described above. Prior to treating the SH-SY5Y cells, BV-2 cells were seeded into 12 well plates at a concentration of 100,000 cells $/ \mathrm{mL}$ and allowed to attach for $24 \mathrm{~h}$. Following attachment, BV-2 cells were exposed to experimental compounds (urolithins at $10 \mu \mathrm{M}$; RESV at $20 \mu \mathrm{M}$ ) for $1 \mathrm{~h}$ prior to exposure to LPS $(1 \mu \mathrm{g} / \mathrm{mL})$ for $24 \mathrm{~h}$. The medium was collected and centrifuged at $18,000 \mathrm{rcf}$ for 5 minutes at $4^{\circ} \mathrm{C}$. Following centrifugation, media from SH-SY5Y cells were removed and replaced with conditioned media from the BV-2 cells and left to incubate for $24 \mathrm{~h}$ and $48 \mathrm{~h}$ after which they were subjected to the CTG 2.0 assay to assess cell viability.

\section{Assessment of Caspase 3/7 and 9 Release}

Caspase $3 / 7$ release within BV-2 microglia and SH-SY5Y neurons were determined by the Caspase 3/7 Glo (Promega, Madison, WI, USA) luminescence based assay. Briefly, BV-2 and SHSY5Y cells were seeded at 100,000 cells $/ \mathrm{mL}$ in white walled clear bottom 96 well plates. The BV-

2 cells were allowed to incubate with the experimental compounds (urolithins at $10 \mu \mathrm{M}$; RESV at $20 \mu \mathrm{M}$ ) for $1 \mathrm{~h}$ prior to the addition of $100 \mu \mathrm{M} \mathrm{H}_{2} \mathrm{O}_{2}$ for $6 \mathrm{~h}$. 
SH-SY5Y cells were exposed to experimental compounds for 24 h prior to $\mathrm{H}_{2} \mathrm{O}_{2}(100 \mu \mathrm{M})$ exposure for $6 \mathrm{~h}$. Following this incubation period, the Caspase 3/7 or 9 Glo reagent and substrate were added in a 1:1 ratio directly to each well. The plate was mixed for approximately 30 seconds on an orbital shaker prior to incubation at room temperature for 35 minutes. Luminescence was recorded using a spectrophotometer (Spectramax M2; Molecular Devices, Sunnyvale, CA, USA).

Measurement of Production of Nitric Oxide Species (NOS)

BV-2 cells, in 24 well plates $(n=4)$ at $85 \%$ confluency $(100,000$ cells $/ \mathrm{mL})$, were serum-starved for $4 \mathrm{~h}$ prior to the treatments. Then the cells were incubated with urolithins $(0.5,1,5$ and $10 \mu \mathrm{M})$ or RESV $(20 \mu \mathrm{M})$ for $1 \mathrm{~h}$ before lipopolysaccharide (LPS) was added to induce an inflammatory response. After $23 \mathrm{~h}$ incubation, the cell culture media were collected and centrifuged. The supernatants were measured for total nitric oxide (NO) using the Griess assay kit (Promega, Madison, WI, USA).

Measurements of IL-6, PGE2, and TNF $\alpha$ Levels

Supernatants collected from the NO production experiments (described above) were collected and assayed for inflammatory cytokines including IL-6 (Biolegend, San Diego, CA, USA), PGE 2 (Cayman Chemical, Ann Arbor, MI, USA), and TNF $\alpha$ (Abcam, Cambridge, MA, USA). Quantification of IL-6, PGE2, and TNFa were measured using Enzyme Linked Immunosorbent assay (ELISA) kits based on the respective manufacturer protocols. 


\section{Statistical Analysis}

Statistical analysis was performed using Graphpad Prism (Version 6. Graphpad Software, San Diego, CA, USA). Data are expressed as means \pm standard errors of mean (S.E.M). Each value was the result of at least three independent experiments $(n=3)$. Comparisons between groups were analyzed using one-way analysis of variance (ANOVA) with corrections for multiple comparisons using Dunnett's Test. P values were also determined by the Student's T-test where appropriate. Calculated $P$ values less than 0.05 were determined to be significant.

\section{Results}

\section{Urolithins modulate generation of LPS-induced reactive nitrogen species in BV-2 microglia}

The effects of urolithins (UA, UB, mUA, mUB) on neuroinflammation were evaluated in an LPSstimulated murine BV-2 microglia cell model (Fig. 1). The urolithins were evaluated at a highest concentration of $10 \mu \mathrm{M}$, which was non-toxic to the cells as previously reported for human neuroblastoma cells, ${ }^{15}$ and also at lower concentrations of 5, 1, and $0.5 \mu \mathrm{M}$. LPS treatment increased nitric oxide (NO) concentration in media by more than two-fold (36 vs. $16 \mu \mathrm{M}$, respectively). Treatment with $10 \mu \mathrm{M}$ of $\mathrm{UA}, \mathrm{UB}, \mathrm{mUA}$ and mUB significantly modulated the release of NO by $46.2,23.5,37.3$, and $34.5 \%$, respectively. Similarly, at $5 \mu M, U A, U B$, mUA and mUB also significantly lowered NO release by $37.4,14.5,23.1$, and $19.6 \%$, respectively. 
The well-known neuroprotective polyphenol, resveratrol (RESV), used as a comparative positive control, decreased NO levels similar to UA, but at a much higher concentration i.e. $45.5 \%$ inhibition achievable with $20 \mu \mathrm{M}$ of RESV which was in agreement as previously reported. ${ }^{17}$

\section{Urolithins attenuate release of LPS-induced pro-inflammatory markers in BV-2 microglia}

We next evaluated the effects of urolithins $(10 \mu \mathrm{M})$ on LPS-stimulated IL-6 and TNF $\alpha$ induction in media collected from BV-2 microglia. LPS treatment increased media IL-6 and TNF $\alpha$ concentrations by $931.7 \%$ and $94.2 \%$ respectively, with RESV $(20 \mu \mathrm{M})$ reducing TNF $\alpha$ and IL-6 by $32.6 \%(587 \mathrm{pg} / \mathrm{mL})$ and $21.7 \%(2007 \mathrm{pg} / \mathrm{mL})$ respectively. UA markedly decreased media IL-6 concentration by $31.3 \%$, followed by UB (19.3\%), mUB (14.6\%), and mUA (8.5\%) (Fig. 2A). Among the urolithins, only UA significantly reduced media TNF $\alpha$ concentration by $75.4 \%(213.8$ pg/mL) (Fig. 2B). LPS increased media PGE 2 concentration by approximately $49.4 \%(519.2$ $\mathrm{pg} / \mathrm{mL}$ ) whereas RESV $(20 \mu \mathrm{M})$ reduced media $\mathrm{PGE}_{2}$ concentration by $29.1 \%(367.9 \mathrm{pg} / \mathrm{mL})$. Among the urolithins, only mUB significantly inhibited media $\mathrm{PGE}_{2}$ concentration by $64.7 \%$ (183.2 pg/mL) compared to $519 \mathrm{pg} / \mathrm{mL}$ of PGE 2 in LPS-treated microglia (Fig. 2C). 


\section{Urolithins mitigate cytotoxicity in a non-contact co-culture model with SH-SY5Y neurons}

Using a non-contact co-culture model as previously reported by our group, ${ }^{16} \mathrm{SH}-\mathrm{SY} 5 \mathrm{Y}$ neuronal cells were exposed to media collected from BV-2 microglia (treated with vehicle control, RESV, urolithins, or LPS alone) and SH-SY5Y cell viability were measured after 24 or 48 h. SH-SY5Y cells exposed to LPS-BV-2 media showed reduced viability as compared to vehicle control at both 24

$\mathrm{h}(16.1 \%$ decrease) and $48 \mathrm{~h}$ ( $48.2 \%$ decrease). The positive control, RESV (at $20 \mu \mathrm{M})$, maintained cell viability with circa $92.5 \%$ viable cells up to $48 \mathrm{~h}$. As shown in Fig. 3A, after $24 \mathrm{~h}$, all of the urolithins (at $10 \mu \mathrm{M}$ ) significantly sustained SH-SY5Y cell viability (>100 \%) greater than the LPS treatment (cell viability circa 80\%). After $48 \mathrm{~h}$ (Fig. 3B), only UA and mUA significantly maintained cell viability ( $92.7 \%$ and $86.5 \%$ viable cells, respectively) relative to that of the LPS treatment (67.7\% viable cells).

\section{Urolithins reduce $\mathrm{H}_{2} \mathrm{O}_{2}$ oxidative stress induced apoptosis and caspases 9 and $3 / 7$ release in}

\section{BV-2 microglia and SH-SY5Y neurons}

Next, we evaluated the protective effects of urolithins on $\mathrm{H}_{2} \mathrm{O}_{2}$ induced oxidative stress of $\mathrm{BV}-2$ microglia and SH-SY5Y neurons. Briefly, BV-2 and SH-SY5Y cells were pre-treated with urolithins (1 $\mathrm{h}$ and $24 \mathrm{~h}$ respectively) after which the cells were exposed to $\mathrm{H}_{2} \mathrm{O}_{2}$ (for $6 \mathrm{~h}$ ). Following this 6 h incubation with $\mathrm{H}_{2} \mathrm{O}_{2}$, cell viability of $\mathrm{BV}-2$ decreased to $68.2 \%$. When pre-treated with RESV $(20 \mu \mathrm{M})$ prior to oxidative insult, RESV afforded an average cell viability of approximately $81.9 \%$. 
As shown in Fig. 4A, the urolithins (at $10 \mu \mathrm{M}$ ) maintained BV-2 cell viability after exposure to $\mathrm{H}_{2} \mathrm{O}_{2}$ as follows: UA (83.6\% viable cells), UB (79.3\% viable cells), mUA (76.5\% viable cells), and mUB (83.1\% viable cells). Similarly, as shown in Fig. 4B, urolithins protected SH-SY5Y cells after exposure to $\mathrm{H}_{2} \mathrm{O}_{2}$ (51.4\% viable cells) with UA maintaining cell viability above $90 \%$ while mUA, mUB, and UB maintained/ increased viability by $12.4,5.8$, and $1.4 \%$, respectively.

We then evaluated the effects of urolithins on caspases 9 and $3 / 7$ release of the BV-2 microglia and $\mathrm{SH}-\mathrm{SY} 5 \mathrm{Y}$ neuronal cells exposed to $\mathrm{H}_{2} \mathrm{O}_{2}$ induced oxidative stress (as described above). $\mathrm{H}_{2} \mathrm{O}_{2}$ caused approximately $129.1 \%$ and $509.6 \%$ increase in caspase 9 activity as compared to untreated vehicle control in BV-2 and differentiated SH-SY5Y, respectively (Fig. 5). Similarly, $\mathrm{H}_{2} \mathrm{O}_{2}$ caused approximately $423.2 \%$ and $121.7 \%$ increase in caspase $3 / 7$ activity as compared to untreated vehicle control in BV-2 and differentiated SH-SY5Y, respectively (Fig. 6). The positive control, RESV (at $20 \mu \mathrm{M})$, also significantly attenuated caspase 9 release in the BV-2 (44.4\% decrease) and SH-SY5Y (62.6\% decrease) cells (Fig. 5), Similarly, RESV (at $20 \mu \mathrm{M}$ ) also significantly attenuated caspase $3 / 7$ release in the BV-2 (46.5\% decrease) and SH-SY5Y (54.4\% decrease) cells (Fig. 6). In BV-2 microglia the highest decrease in caspase 9 activity was observed for mUB (62.9\%) followed by UA (61.3\%), UB (57.8\%), mUA (60.8\%), respectively (Fig. 5A). In SH-SY5Y, the highest decrease in caspase 9 activity was observed for UB (78.8\%) followed by mUA (78.2\%), mUB (75.8\%), UA (67.8\%), respectively (Fig. 5B). 
Among the urolithins, only UB ( $12.0 \%$ decrease) and mUA (17.8\% decrease) significantly attenuated caspase $3 / 7$ release in BV-2 microglia (Fig. 6A). All of the urolithins, except UA, significantly decreased caspase $3 / 7$ release in SH-SY5Y as follows: UB (35.9\%), mUA (59.2\%) and mUB (72.0\%) (Fig. 6B).

Pomegranate extract (PE) appears to follow a decreasing trend in reduction of gene expression of inflammatory biomarkers in excised hippocampus tissues of AD transgenic R1.40 mice

We conducted secondary analyses of excised whole sections of the hippocampus collected from our previously reported ${ }^{11} A D$ transgenic $R 1.40$ mouse study. While not statistically significant, PE treatment (at $200 \mu \mathrm{g} / \mathrm{mL}$ ) appears to follow a decreasing trend in the transcription levels of COX-2, IL-I, TNF $\alpha$, and IL-6 as compared to the control animals (Fig. 7)

\section{Discussion}

Exposure to environmental toxins and infectious agents, along with diet, have a profound impact on neuroinflammation, and consequently, brain health. ${ }^{18}$ Polyphenol-rich plant foods have been reported to inhibit neuroinflammation in both in vitro and in vivo studies ${ }^{19,20}$ and several, including curcumin, ${ }^{21}$ olive oil, ${ }^{22}$ grape, ${ }^{23}$ and Pomegranate, ${ }^{6-11}$ show protective effects against neurodegenerative diseases including AD in cell and rodent models. However, the poor systemic bioavailability coupled with low blood brain barrier penetrability of polyphenols remain as major challenges towards their advancement as dietary agents for AD prevention and/or treatment. $^{24}$ 
Interestingly, the biotransformation of polyphenols by human gut microflora into bioavailable and bioactive gut microbial-derived metabolites has attracted considerable research interest in the context of human health including neurodegenerative diseases. ${ }^{25-27}$ Moreover, polyphenol-derived gut microbial metabolites have been reported to achieve detectable and physiologically relevant levels in circulation and some even cross the blood brain barrier. $^{28}$

As previously noted, the Pomegranate shows neuroprotective effects in several AD animal models, ${ }^{6-11}$ but while its naturally occurring ellagitannins, are not bioavailable, their gut microbial derived metabolites, namely, urolithins, are detectable in circulation ${ }^{25}$ and can potentially cross the blood brain barrier. ${ }^{12,28}$ Our group has previously reported on the neuroprotective effects of a PE which we proposed were largely mediated by urolithins rather than the naturally occurring Pomegranate ellagitannins. ${ }^{11,12}$ While recently urolithins have been evaluated for their anti-inflammatory effects in human neuroblastoma SH-SY5Y cells, ${ }^{15}$ there have been no similar studies in human neuronal SH-SY5Y cells (achieved by differentiating the neuroblastoma SH-SY5Y cells) nor in microglia cells with these compounds. Also, while punicalagin, a major Pomegranate ellagitannin has been reported to show anti-apoptotic effects of rat PC12 cells by mitigating oxidative stress, ${ }^{42}$ studies on the relevant circulating compounds, namely, the urolithins, are necessary. Therefore, based on our hypothesis that urolithins mediate the neuroprotective effects of PE, secondary analyses of the hippocampal tissues collected from our previous $A D$ animal study, ${ }^{11}$ appeared to show a decreasing trend in gene expression of several inflammatory biomarkers. 
Variability from mouse to mouse was a confounding factor in that study warranting further studies using a larger sample size of animals. Also, unfortunately, because of limited brain tissues remaining from that study we were unable to isolate protein to conduct western blot analyses for protein expression. Moreover, we lack direct evidence confirming the presence of urolithins in brain tissues (for e.g., achievable by analytical methods such as mass spectrometry) collected from the transgenic AD animals. Thus, our group's future planned studies include conducting additional animal studies using relevant doses of purified urolithins to elucidate their pharmacokinetics, pharmacodynamics, and mechanism/s of action. Notably, UA was recently reported to induce auto/mitophagy in vitro and in vivo which may be an important consideration in the neuroprotective effects of these compounds. ${ }^{29}$

Next, we designed a series of in vitro studies using BV-2 microglia and SH-SY5Y neuronal cells to explore the effects of the urolithins against neuroinflammation. The mechanisms of cell death, specifically within neurons, are well documented and attributable to oxidative stress by radical species via the intrinsic apoptosis pathway. ${ }^{30,31}$ Moreover, the exposure of neurons to damage and pathogen associated proteins/markers directly from neighboring cells (for e.g. microglia and astrocytes) are involved in the initiation of the extrinsic pathway of apoptosis. ${ }^{32}$ Intervention in these pathways or the modulation of the production of pro-inflammatory markers are potential methods for treating complex neurodegenerative diseases. On this basis, we have shown that urolithins may prevent neuronal cell death by preventing the generation of reactive nitrogen species and major pro-inflammatory cytokines including IL-6, PGE 2 , and TNF $\alpha$. These inflammatory biomarkers have been implicated in vitro and in vivo in the production of $\beta$ amyloid and neurofibrillary tangles. ${ }^{33}$ 
To evaluate the effects of urolithins on oxidative stress, we used hydrogen peroxide $\left(\mathrm{H}_{2} \mathrm{O}_{2}\right)$, a well-known inducer of the intrinsic oxidative stress induced apoptosis pathway, to evaluate the protective effects of the compounds on microglia and neurons. ${ }^{34-36}$ Moreover, we probed the penultimate and ultimate checkpoint proteases responsible for cell death, namely, caspases 9, 3/7 respectively. Cysteinyl-aspartate proteases (caspases) are the executioner proteins of a dying cell which act on DNA repair enzymes ultimately leading to DNA fragmentation and cell death. This activation not only represents the final step in apoptosis but occurs in the microglia of individuals with AD-like conditions when exposed to either oxidative stress via free radicals or pro-inflammatory molecules from activated microglia or dying cells. ${ }^{37}$ Caspases 3 and 7 are the major caspase isoforms involved in apoptosis and thus represent possible therapeutic targets to prolong cell life. ${ }^{37}$

Thus, by pre-treating BV-2 murine microglia with urolithins and then inducing oxidative stress in these cells with $\mathrm{H}_{2} \mathrm{O}_{2}$, the extent of caspases 3 and 7 release were measured. Our findings show that urolithins mitigated acute oxidative stress within microglia and neurons by maintaining cell viability and mitigating caspases 3 and 7 activity. Upstream of the caspase mediated intrinsic apoptosis pathway, effector caspase 9 activity was also diminished by urolithins. Taken together, urolithins may exert neuroprotective effects by preventing oxidative stress mediated apoptosis by preventing caspase activation. Moreover, we also showed the effects of urolithins on microglial modulation using a non-contact co-culture cell model with differentiated SH-SY5Y neurons as previously reported by our group. ${ }^{16}$ 
Indeed, media from the urolithins treated BV-2-LPS cells increased cell viability of SHSY5Y cells greater than media collected from non-urolithin treated BV-2-LPS cells. A limitation of this assay includes the origin of cells that were used, namely, microglia and neuronal cells of murine and human origin, respectively. While it would have been more ideal to use human instead of murine microglia, it should be noted that human microglial cells are restricted to limited number of passages before they begin to undergo drastic morphological changes. Also, the secretome of murine microglia differs from human microglia and responsiveness of BV-2 murine microglia to certain immunostimulatory molecules may also be inconsistent with their human counterpart. ${ }^{38}$ Another limitation of our study could arise from the differentiation process of converting human SH-SY5Y neuroblastoma cells into neuronal cells (using retinoic acid for a period of 7 days). It is possible that incomplete differentiation of the cells could lead to variability in sensitivity to neurotrophins/neurotoxins, ${ }^{39}$ viability, and ultimately, protein expression. $^{36}$

In the in vitro experiments, we chose the well-known polyphenol, namely, resveratrol (RESV) as a comparative positive control. However, there is considerable variation reported in RESV concentrations which are required to achieve 'neuroprotective' effects in SH-SY5Y, BV-2, and other similar cells. ${ }^{40,41}$ Herein, we evaluated RESV at $20 \mu \mathrm{M}$ since higher concentrations were toxic to the BV-2 and SH-SY5Y cells while lower concentrations were not neuroprotective. In contrast, the urolithins were all evaluated at a non-toxic dose of $10 \mu \mathrm{M}$ which was in agreement with a previous study conducted in neuroblastoma cells. ${ }^{15}$ 
Thus, while further animal studies are required to ascertain whether circulating urolithins can achieve these concentrations in the brain, the current study provides useful mechanistic data supporting the anti-neuroinflammatory effects of urolithins, which may contribute to the overall neuroprotective effects reported for Pomegranate.

\section{Conclusions}

Although the pathogenesis of $A D$ is still under discussion, the link between neuroinflammation and neurodegenerative diseases has been well established. Numerous studies have shown that long term exposure to toxicants, frequent infections, and poor diet can negatively impact the blood brain barrier, increasing the risk of microglial activation and thus increasing the secretion of pro-inflammatory molecules that would ultimately lead to the premature termination of neuronal and astrocytic cells. Intervention during this microglial activation process has become an attractive target in the disruption of the pathogenesis of AD. Other targets for this disease include $A \beta$ export/ablation drugs in addition to acetylcholinesterase inhibitors. Currently, the available drugs that are administered to patients are only palliative and thus do not address the underlying cause of this disease. However, natural products, including certain berries and fruit such as Pomegranate, show promise as dietary intervention strategies for AD prevention and/or treatment. In summary, herein, we report that PE-treated animals appeared to show decreasing trend in gene expression of inflammatory cytokines in brain tissues in vivo and that urolithins prevent the over-activation of microglia and maintain viability of neurons in vitro. Further investigation into brain penetrability and the molecular target/s of urolithins are warranted in order to elucidate their specific pathway/s of action and their potential preventive and/or therapeutic impact on $A D$ pathogenesis. 


\section{Disclaimer statements}

\section{Conflict of Interests}

The authors declare no conflicts of interest.

\section{Ethics approval}

Animals were used in compliance with all applicable laws and regulations and with the principles expressed by the NIH Guide for the Care and Use of Laboratory Animals. 


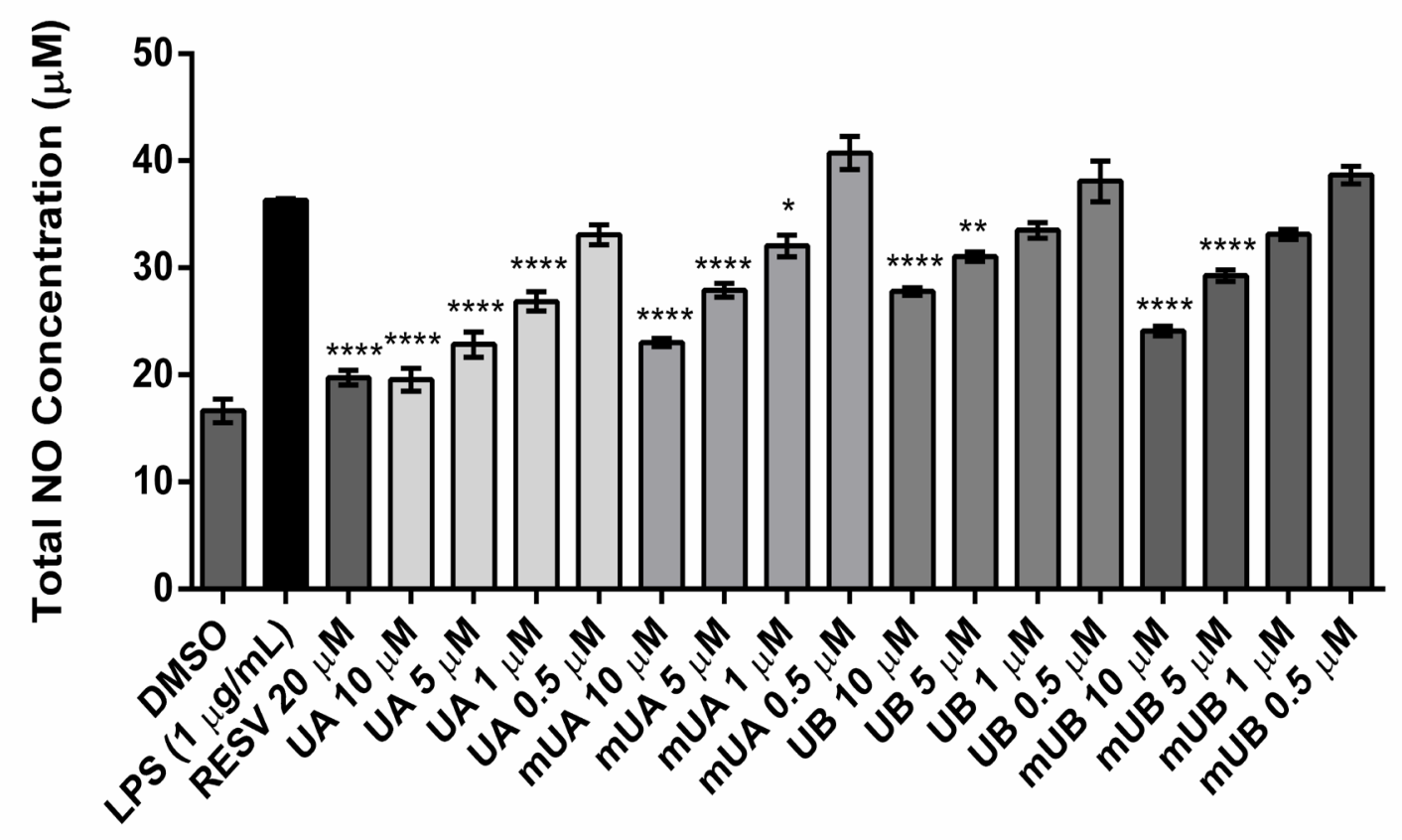

Figure 1 Urolithins attenuate LPS-stimulated elevation of media nitric oxide (NO) in murine microglial cells. Pre-treatment of microglia with vehicle, resveratrol (RESV), or urolithins for $1 \mathrm{~h}$ was followed by a $24 \mathrm{~h}$ incubation period with LPS. Release of NO (as measured by the Griess assay) was attenuated by urolithins at concentrations from 1-10 $\mu \mathrm{M}$. Data expressed as mean \pm standard error $(n=4)$. Comparisons are made to LPS where $P<0.05^{*}, P<0.01 * *, P<0.001 * * *$, $P<0.0001 * * * *$. 


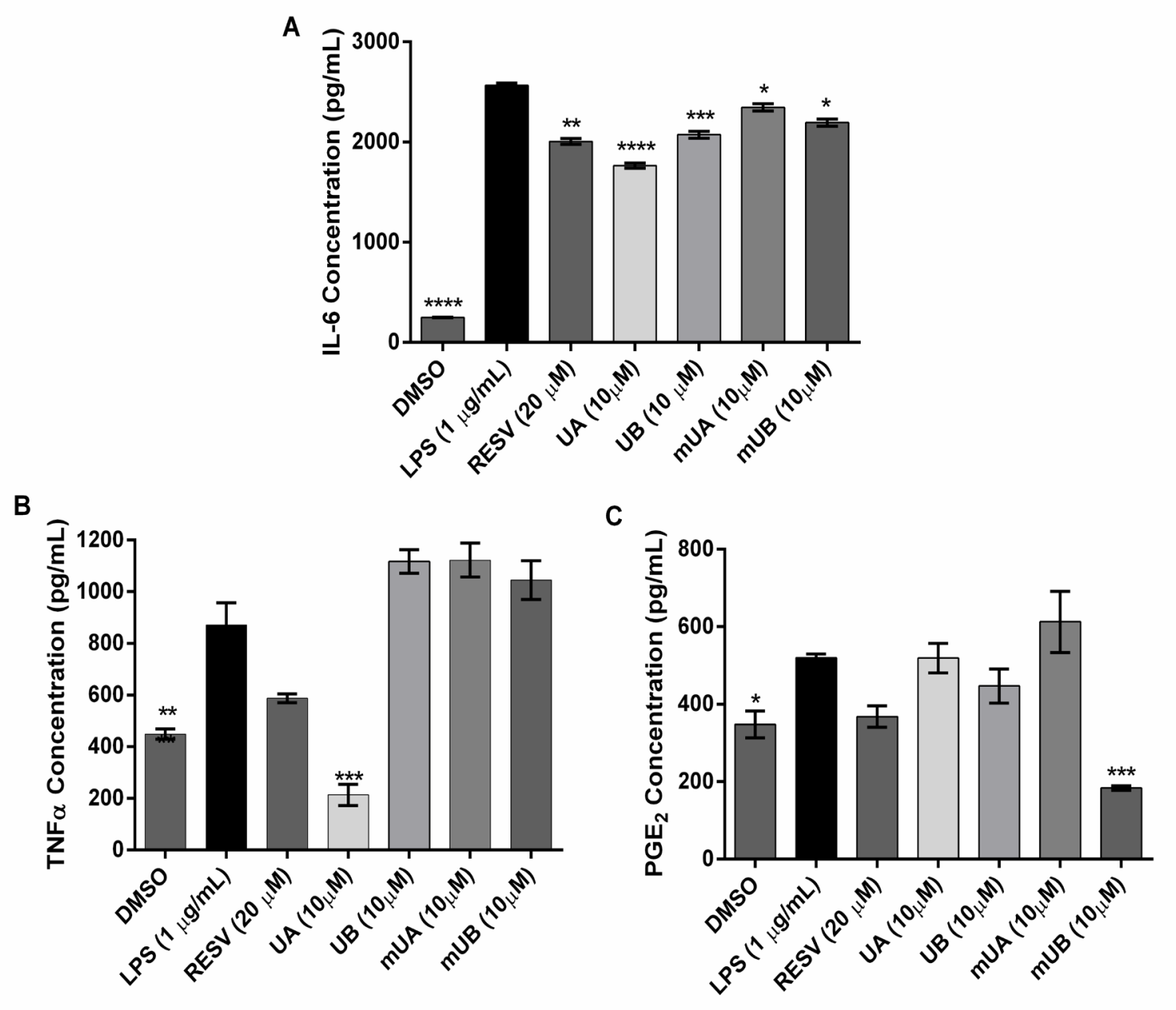

Figure 2 Urolithins modulate the release of pro-inflammatory cytokines in LPS stimulated murine BV-2 microglia. BV-2 microglia were pre-treated with vehicle, urolithins, or resveratrol (RESV) prior to LPS exposure for 24 hours. When incubated for $1 \mathrm{~h}$ prior to LPS stimulation, urolithins modulated the secretion of IL-6 $(A)$ in cell culture media. UA significantly inhibited the release of TNFa (B) whereas only $m U B$ significantly inhibited $P G E_{2}$ levels (C). Data expressed as mean \pm standard error ( $n=4)$. Comparisons are made to LPS where $P<0.05^{*}, P<0.01 * *, P<$ $0.001 * * *, P<0.0001 * * * *$. 
Viability $24 \mathrm{hrs}$

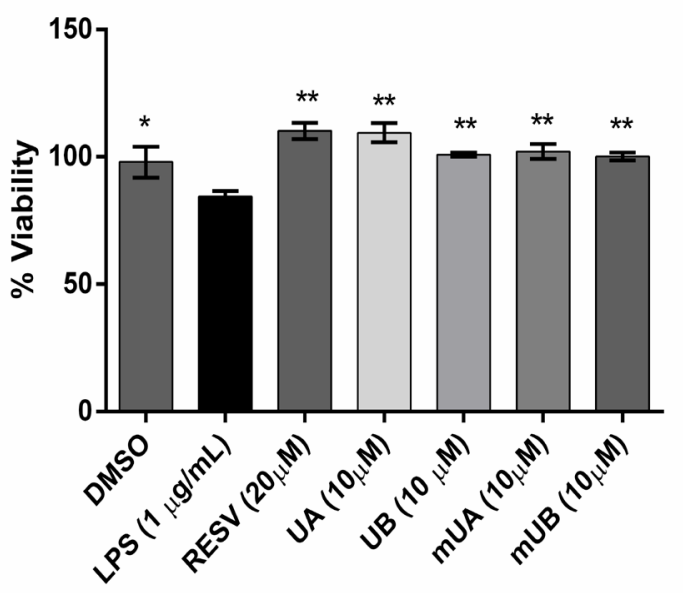

Viability $48 \mathrm{hrs}$

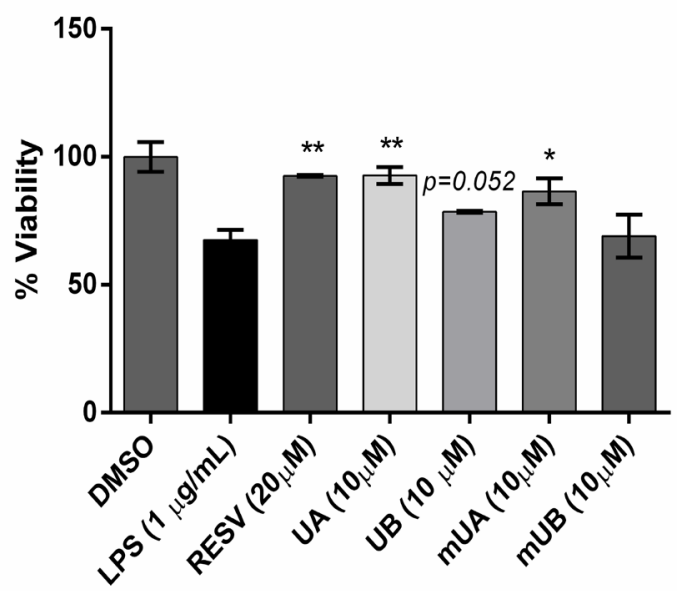

Figure 3 Urolithins maintain human neuronal SH-SY5Y cell viability when exposed to LPS conditioned media from microglia. BV-2 microglia were pre-treated with vehicle, urolithins, or resveratrol (RESV) prior to LPS exposure for 24 hours. LPS conditioned media was then centrifuged and introduced to differentiated SH-SY5Y cells. Viability of differentiated SH-SY5Y cells was significantly sustained after 24 and 48 hours of urolithins as compared to the viability of cells containing conditioned BV-2 -LPS media. Data expressed as mean \pm standard error $(n=3)$. Comparisons are made to LPS where $P<0.05^{*}, P<0.01^{* *}, P<0.001 * * *, P<0.0001 * * * *$. 

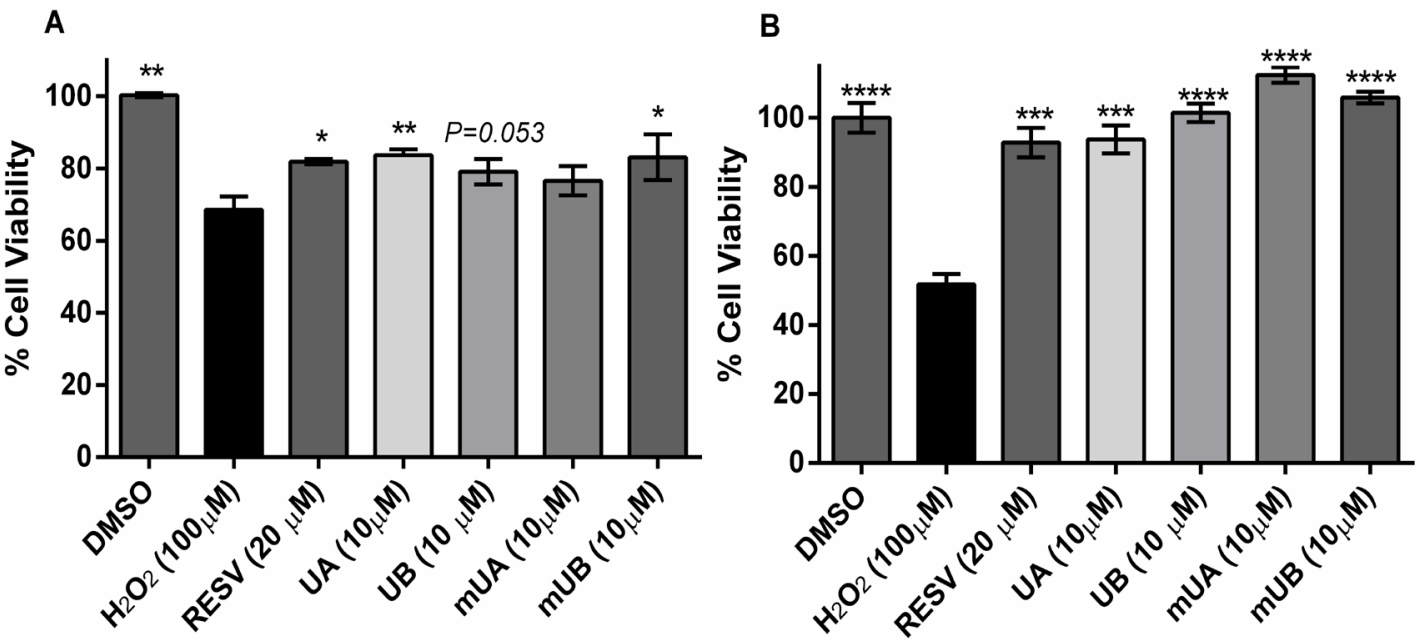

Figure 4 Urolithins maintain BV-2 murine microglia and SH-SY5Y human neuron cell viability. BV-2 microglia were pre-treated with vehicle, urolithins, or resveratrol (RESV) prior to LPS exposure for 24 hours. When exposed to the intrinsic oxidative stressor hydrogen peroxide $\left(\mathrm{H}_{2} \mathrm{O}_{2}\right)$ for 6 hours following either a 1 hour or 24 hour pre-treatment of urolithins, cell viability is significantly improved in both BV-2 murine microglia (4A) and in differentiated human neuroblastoma SH-SY5Y (4B) Data expressed as mean \pm standard error $(n=4)$. Comparisons are made to $\mathrm{H}_{2} \mathrm{O}_{2}$ where $\mathrm{P}<0.05 *, P<0.01 * *, P<0.001 * * *, P<0.0001 * * * *$. 

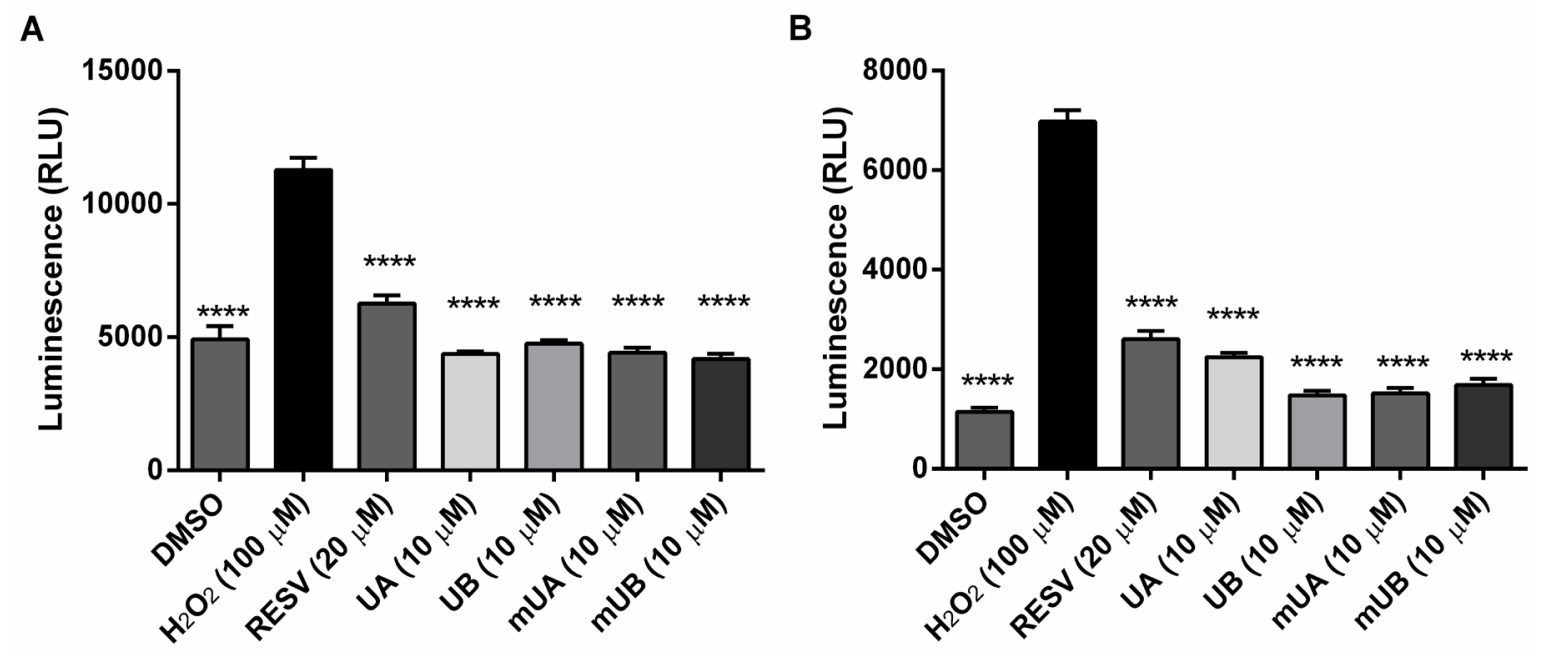

Figure 5 Hydrogen peroxide induced caspase 9 protease activity is decreased in murine microglia (BV-2) and differentiated human neuroblastoma. Urolithins prevent the onset of intrinsic pathway apoptotic events by diminishing caspase 9 activity after incubating with BV-2 for 1 hour (5A) and RA differentiated SH-SY5Y for 24 hours (5B) prior to $\mathrm{H}_{2} \mathrm{O}_{2}$ exposure for 6 hours. Data expressed as mean \pm standard error $(n=4)$. Comparisons are made using $\mathrm{H}_{2} \mathrm{O}_{2}$ where $P<0.05^{*}, P<0.01 * *, P<0.001 * * *, P<0.0001 * * * *$. 
A

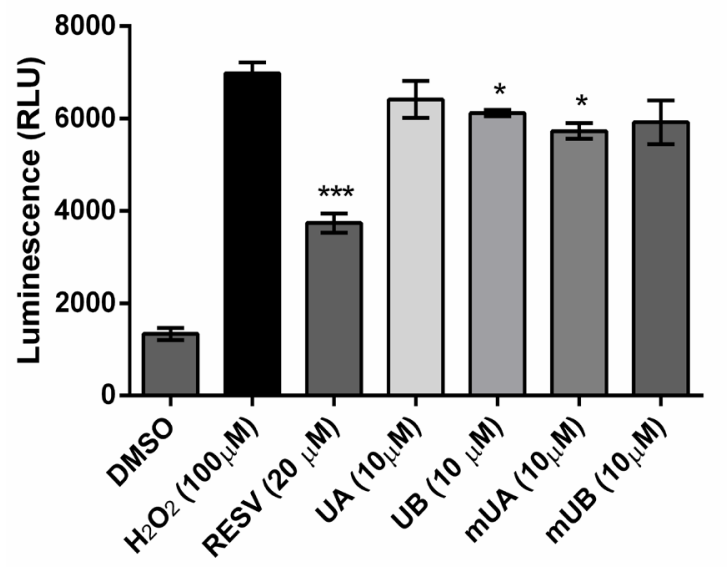

B

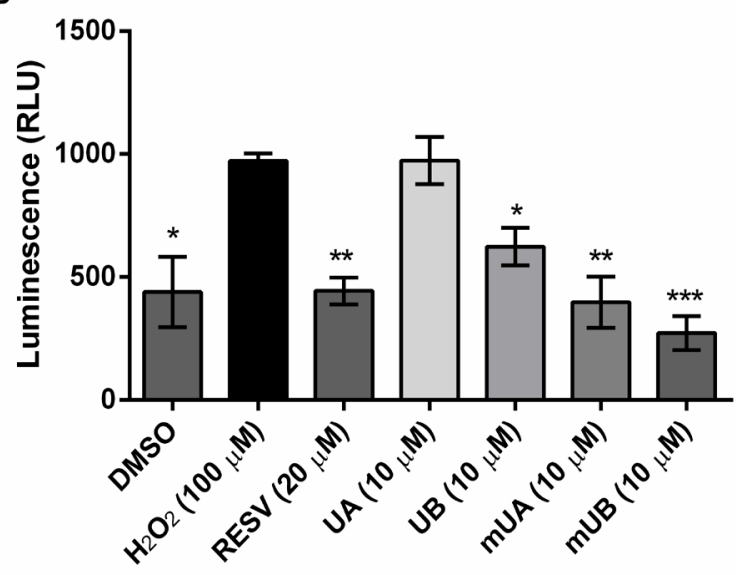

Figure 6 Hydrogen peroxide induced caspase $3 / 7$ release is abrogated in murine microglia (BV-2) and human neurons.

Urolithins maintain cell viability and prevent the release of caspase $3 / 7$ after incubating with BV2 for 1 hour (6A) and $\mathrm{SH}$-SY5Y for 24 hours (6B) prior to $\mathrm{H}_{2} \mathrm{O}_{2}$ exposure for 6 hours. Data expressed as mean \pm standard error $(n=4)$. Comparisons are made using $\mathrm{H}_{2} \mathrm{O}_{2}$ where $P<0.05 *$, $P<0.01 * *, P<0.001 * * *, P<0.0001 * * * *$. 
A

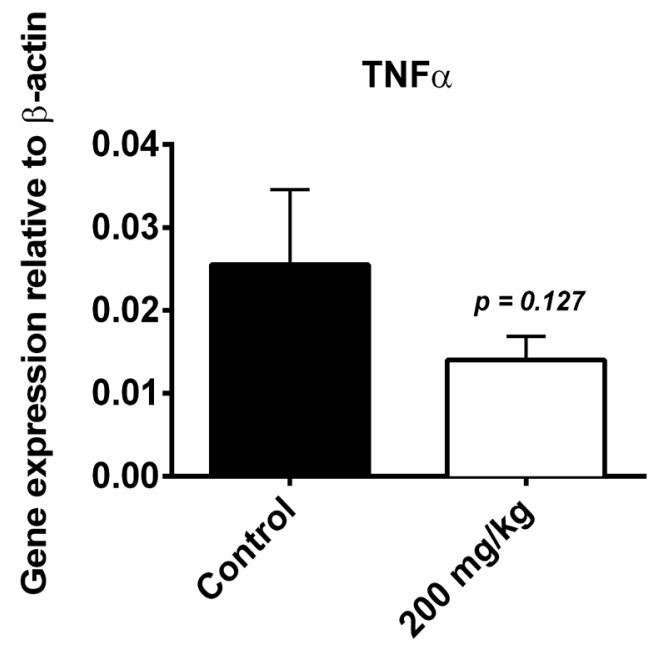

C

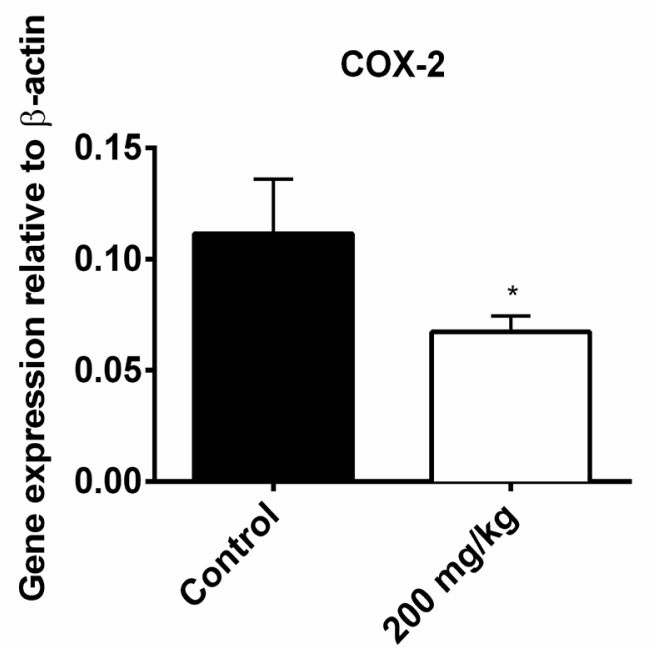

B

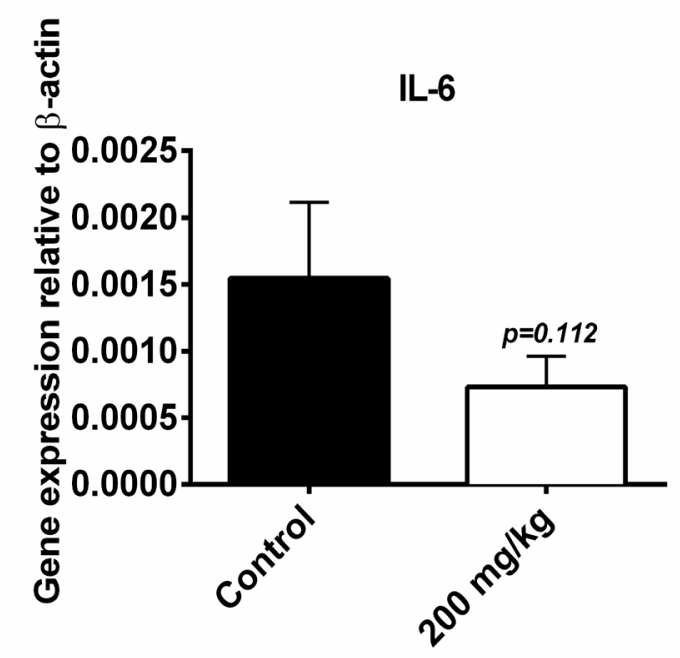

D

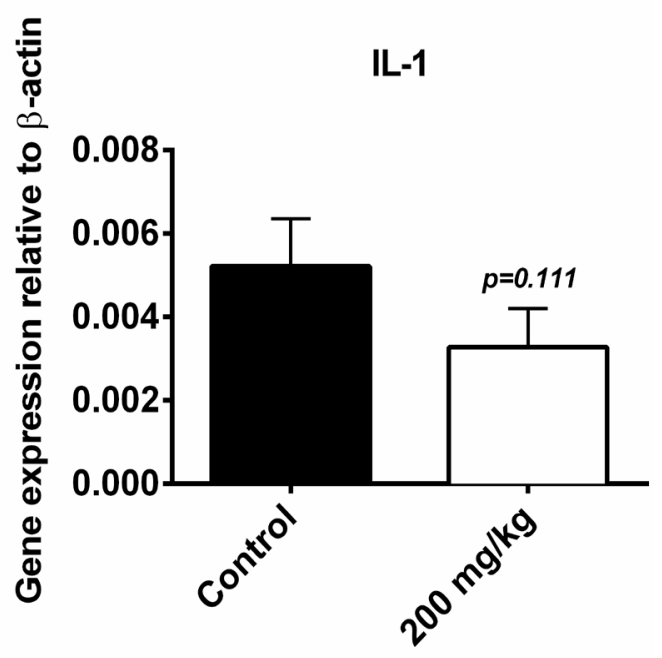

Figure 7 Pomegranate extract (PE) mitigates release of pro-inflammatory cytokines in hippocampi of transgenic mice fed PE. Administration of PE to transgenic R1.40 AD mice at 200 $\mathrm{mg} / \mathrm{kg}$ appeared to show a decreasing trend in TNF $\alpha(A)$, IL-6 (B), COX-2 (C), and IL-1 (D) expressed within the hippocampus. Comparisons are made using control diet fed mice where $\mathrm{P}<$ $0.05 *$ using a student t-test. 


\section{References}

1. Heneka MT, Carson MJ, El Khoury J, Landreth GE, Brosseron F, Feinstein DL, et al. Neuroinflammation in Alzheimer's disease. Lancet Neurol 2015;14:388-405.

2. Streit WJ, Mrak RE, Griffin WST. Microglia and neuroinflammation: a pathological perspective. J Neuroinflammation 2004;1:1.

3. Smith JA, Das A, Ray SK, Banik NL. Role of pro-inflammatory cytokines released from microglia in neurodegenerative diseases. Brain Res Bull 2012;87:10-20.

4. Streit WJ, Walter SA, Pennell NA. Reactive microgliosis. Prog Neurobiol 1999;57:563581.

5. Espín JC, Larrosa M, García-Conesa MT, Tomás-Barberán F. Biological significance of urolithins, the gut microbial ellagic acid-derived metabolites: the evidence so far. Evid Based Complement Alternat Med 2013. 1

6. Choi SJ, Lee J-H, Heo HJ, Cho HY, Kim HK, Kim C-J, et al. Punica granatum protects against oxidative stress in PC12 cells and oxidative stress-induced Alzheimer's symptoms in mice. J Med Food 2011;14:695-701.

7. Hartman RE, Shah A, Fagan AM, Schwetye KE, Parsadanian M, Schulman RN, et al. Pomegranate juice decreases amyloid load and improves behavior in a mouse model of Alzheimer's disease. Neurobiol Dis 2006;24:506-515.

8. Rojanathammanee L, Puig KL, Combs CK. Pomegranate polyphenols and extract inhibit nuclear factor of activated T-cell activity and microglial activation in vitro and in a transgenic mouse model of Alzheimer disease. J Nutr 2013;143:597-605.

9. Subash S, Essa MM, Al-Asmi A, Al-Adawi S, Vaishnav R, Braidy N, et al. Pomegranate from Oman alleviates the brain oxidative damage in transgenic mouse model of alzheimer's disease. J Trad Complement Med 2014;4:232-238.

10. Subash S, Braidy N, Essa MM, Zayana A-B, Ragini V, Al-Adawi S, et al. Long-term (15 mo) dietary supplementation with Pomegranates from Oman attenuates cognitive and behavioral deficits in a transgenic mice model of Alzheimer's disease. Nutrition 2015;31:223-229.

11. Ahmed AH, Subaiea GM, Eid A, Li L, Seeram NP, Zawia NH. Pomegranate extract modulates processing of amyloid- $\beta$ precursor protein in an aged Alzheimer's disease animal model. Curr Alzheimer Res 2014;11:834-843.

12. Yuan T, Ma H, Liu W, Niesen DB, Shah N, Crews R, et al. Pomegranate's neuroprotective effects against Alzheimer's disease are mediated by urolithins, its ellagitannin-gut microbial derived metabolites. ACS Chem Neurosci 2016;7:26-33. 
13. Adams LS, Zhang Y, Seeram NP, Heber D, Chen S. Pomegranate ellagitannin-derived compounds exhibit antiproliferative and antiaromatase activity in breast cancer cells in vitro. Cancer Prevent Res 2010;3:108-113.

14. Núñez-Sánchez MÁ, Karmokar A, González-Sarrías A, García-Villalba R, Tomás-Barberán FA, García-Conesa MT, et al. In vivo relevant mixed urolithins and ellagic acid inhibit phenotypic and molecular colon cancer stem cell features: A new potentiality for ellagitannin metabolites against cancer. Food Chem Toxicol 2016;92:8-16.

15. Gonzalez-Sarrias A, Núñez-Sánchez MÁ, Tomas-Barberan FA, Espín JC. Neuroprotective effects of bioavailable polyphenol-derived metabolites against oxidative stress-induced cytotoxicity in human neuroblastoma SH-SY5Y cells. J Agric Food Chem 2016. DOI: 10.1021/acs.jafc.6b04538

16. Ma H, DaSilva NA, Liu W, Nahar PP, Wei Z, Liu Y, et al. Effects of a standardized phenolicenriched maple syrup extract on $\beta$-amyloid aggregation, neuroinflammation in microglial and neuronal cells, and $\beta$-amyloid induced neurotoxicity in Caenorhabditis elegans. Neurochem Res 2016;41:2836-2847.

17. Carey AN, Fisher DR, Rimando AM, Gomes SM, Bielinski DF, Shukitt-Hale B. Stilbenes and anthocyanins reduce stress signaling in BV-2 mouse microglia. Journal of agricultural and food chemistry. 2013;61:5979-5986.

18. García-Bueno B, Caso JR, Leza JC. Stress as a neuroinflammatory condition in brain: damaging and protective mechanisms. Neurosci Biobehav Rev 2008;32:1136-1151.

19. Galli RL, Shukitt-Hale B, Youdim KA, Joseph JA. Fruit polyphenolics and brain aging. Ann N Y Acad Sci 2002;959:128-132.

20. Lau FC, Shukitt-Hale B, Joseph JA. The beneficial effects of fruit polyphenols on brain aging. Neurobiol Aging. 2005;26:128-132.

21. Ringman JM, Frautschy SA, Cole GM, Masterman DL, Cummings JL. A potential role of the curry spice curcumin in Alzheimer's disease. Curr Alzheimer Res 2005;2:131-136.

22. Abuznait AH, Qosa H, Busnena BA, El Sayed KA, Kaddoumi A. Olive-oil-derived oleocanthal enhances $\beta$-amyloid clearance as a potential neuroprotective mechanism against Alzheimer's disease: in vitro and in vivo studies. ACS Chem Neurosci 2013;4:973-982.

23. Wang J, Santa-Maria I, Ho L, Ksiezak-Reding H, Ono K, Teplow DB, et al. Grape derived polyphenols attenuate tau neuropathology in a mouse model of Alzheimer's disease. J Alzheimer's Dis 2010;22:653-661.

24. Singh $M$, Arseneault $M$, Sanderson $T$, Murthy $V$, Ramassamy $C$. Challenges for research on polyphenols from foods in Alzheimer's disease: bioavailability, metabolism, and cellular and molecular mechanisms. J Agric Food Chem 2008;56:4855-4873. 
25. Selma MV, Espin JC, Tomas-Barberan FA. Interaction between phenolics and gut microbiota: role in human health. J Agric Food Chem 2009;57:6485-6501.

26. Ebrahimi A, Schluesener H. Natural polyphenols against neurodegenerative disorders: potentials and pitfalls. Ageing Res Rev 2012;11:329-345.

27. Verzelloni E, Pellacani C, Tagliazucchi D, Tagliaferri S, Calani L, Costa LG, et al. Antiglycative and neuroprotective activity of colon-derived polyphenol catabolites. Mol Nutr Food Res 2011;55:S35-S43.

28. Gasperotti M, Passamonti S, Tramer F, Masuero D, Guella G, Mattivi F, et al. Fate of microbial metabolites of dietary polyphenols in rats: is the brain their target destination? ACS Chem Neurosci 2015;6:1341-1352.

29. Ryu D, Mouchiroud L, Andreux PA, Katsyuba E, Moullan N, Nicolet-dit-Félix AA, et al. Urolithin A induces mitophagy and prolongs lifespan in $C$. elegans and increases muscle function in rodents. Nature Med 2016;22:879-888.

30. Patten DA, Germain M, Kelly MA, Slack RS. Reactive oxygen species: stuck in the middle of neurodegeneration. J Alzheimer's Dis 2010;20:357-367.

31. Lacoste MG, Ponce IT, Golini RL, Delgado SM, Anzulovich AC. Aging modifies daily variation of antioxidant enzymes and oxidative status in the hippocampus. Exp Gerontol 2016. DOI: 10.1016/j.exger.2016.12.002

32. Murray PJ, Wynn TA. Protective and pathogenic functions of macrophage subsets. Nat Rev Immunol 2011;11:723-737.

33. Hoshino T, Suzuki K, Matsushima T, Yamakawa N, Suzuki T, Mizushima T. Suppression of Alzheimer's disease-related phenotypes by geranylgeranylacetone in mice. PLoS One 2013;8:e76306.

34. Ismail N, Ismail M, Farhana Fathy S, Asma Musa SN, Umar Imam M, Foo JB, et al. Neuroprotective effects of germinated brown rice against hydrogen peroxide induced cell death in human SH-SY5Y cells. Int J Mol Sci 2012;13:9692-9708.

35. Facchinetti $\mathrm{F}$, Furegato $\mathrm{S}$, Terrazzino $\mathrm{S}$, Leon $\mathrm{A} . \mathrm{H}_{2} \mathrm{O}_{2}$ induces upregulation of Fas and Fas ligand expression in NGF-differentiated PC12 cells: Modulation by cAMP. J Neurosci Res 2002;69:178-188.

36. Ruffels J, Griffin M, Dickenson JM. Activation of ERK1/2, JNK and PKB by hydrogen peroxide in human SH-SY5Y neuroblastoma cells: role of ERK1/2 in $\mathrm{H}_{2} \mathrm{O}_{2}$-induced cell death. Euro J Pharmacol 2004;483:163-173.

37. Burguillos MA, Deierborg T, Kavanagh E, Persson A, Hajji N, Garcia-Quintanilla A, et al. Caspase signalling controls microglia activation and neurotoxicity. Nature 2011;472:319-324. 
38. Stansley B, Post J, Hensley K. A comparative review of cell culture systems for the study of microglial biology in Alzheimer's disease. J Neuroinflammation 2012;9:1.

39. Jämsä A, Hasslund K, Cowburn RF, Bäckström A, Vasänge $M$. The retinoic acid and brainderived neurotrophic factor differentiated SH-SY5Y cell line as a model for Alzheimer's diseaselike tau phosphorylation. Biochem Biophys Res Commun 2004;319:993-1000.

40. Currò M, Trovato-Salinaro A, Gugliandolo A, Koverech G, Lodato F, Caccamo D, et al. Resveratrol protects against homocysteine-induced cell damage via cell stress response in neuroblastoma cells. J Neurosc Res 2015;93:149-156.

41. Lin T-K, Chen S-D, Chuang Y-C, Lin H-Y, Huang C-R, Chuang J-H, et al. Resveratrol partially prevents rotenone-induced neurotoxicity in dopaminergic SH-SY5Y cells through induction of heme oxygenase-1 dependent autophagy. Int J Mol Sci 2014;15:1625-1646.

42. Clementi ME, Pani G, Sampaolese B, Tringali G. Punicalagin reduces H2O2-induced cytotoxicity and apoptosis in PC12 cells by modulating the levels of reactive oxygen species. Nutr Neurosci 2017 Apr 9:1-8. doi: 10.1080/1028415X.2017.1306935. 


\section{MANUSCRIPT 5}

Published Neurochemical Research 2016 Nov;41(11):2836-2847

Effects of a Standardized Phenolic-Enriched Maple Syrup Extract on $\beta$-Amyloid Aggregation, Neuroinflammation in Microglial and Neuronal Cells, and $\beta$-Amyloid Induced Neurotoxicity in Caenorhabditis elegans

Hang $\mathrm{Ma}^{1}$, Nicholas A. DaSilva ${ }^{1}$, Weixi $\mathrm{Liu}^{1}$, Pragati P. Nahar ${ }^{1}$, Zhengxi Wei ${ }^{1}$, Yongqiang $\mathrm{Liu}^{1}$, Priscilla T. Pham ${ }^{2}$, Rebecca Crews ${ }^{2}$, Dhiraj A. Vattem ${ }^{2}$, Angela L. Slitt ${ }^{1}$, Zahir A. Shaikh ${ }^{1}$, Navindra P. Seeram ${ }^{1 *}$

${ }^{1}$ Department of Biomedical and Pharmaceutical Sciences, College of Pharmacy, University of Rhode Island, Kingston, RI 02881, United States

${ }^{2}$ Nutrition Biomedicine and Biotechnology, Texas State University, San Marcos, TX 78666, United States

*Author to whom correspondence should be addressed:

N.P.S.: Phone/Fax: 401-874-9367/5787; Email: nseeram@uri.edu 


\section{Abbreviations}

Alzheimer's disease (AD); amyloid-beta $(A \beta)$; phenolic-enriched maple syrup extract (MSX); Thioflavin T (ThT); transmission electron microscopy (TEM); circular dichroism (CD); dynamic light scattering (DLS); amyloid peptide 1-42 (A $\left.\beta_{1-42}\right) ; 2^{\prime}, 7^{\prime}$-dichlorofluorescin diacetate (DCFDA); lipopolysaccharide (LPS); resveratrol (RESV); Dulbecco's modified eagle medium: nutrient mixture F-12 (DMEM/F12); fetal bovine serum (FBS); dimethyl sulfoxide (DMSO); [3-(4,5dimethylthiazol-2-yl)-2,5-diphenyltetrazolium bromide] (MTT); CellTiter-Glo (CTG); reactive oxygen species (ROS); nitric oxide species (NOS); nitric oxide (NO); interleukin-6 (IL-6); prostaglandin E2 ( $\left.\mathrm{PGE}_{2}\right)$; tumor necrosis factor $\alpha(\mathrm{TNF} \alpha)$; enzyme linked immunosorbent assay (ELISA); high performance liquid chromatography-diode array detection (HPLC-DAD); gallic acid equivalents (GAEs). 


\section{Abstract}

Published data supports the neuroprotective effects of several phenolic-containing natural products, including certain fruit, berries, spices, nuts, green tea, and olive oil. However, limited data are available for phenolic-containing plant-derived natural sweeteners including maple syrup. Herein, we investigated the neuroprotective effects of a chemically standardized phenolic-enriched maple syrup extract (MSX) using a combination of biophysical, in vitro, and in vivo studies. Based on biophysical data (Thioflavin T assay, transmission electron microscopy, circular dichroism, dynamic light scattering, and zeta potential), MSX reduced amyloid $\beta_{1-42}$ peptide $\left(A \beta_{1-42}\right)$ fibrillation in a concentration-dependent manner $(50-500 \mu \mathrm{g} / \mathrm{mL})$ with similar effects as the neuroprotective polyphenol, resveratrol, at its highest test concentration (63.5\% at $500 \mu \mathrm{g} / \mathrm{mL}$ vs. $77.3 \%$ at $50 \mu \mathrm{g} / \mathrm{mL}$, respectively). MSX $(100 \mu \mathrm{g} / \mathrm{mL})$ decreased $\mathrm{H}_{2} \mathrm{O}_{2}$-induced oxidative stress ( $16.1 \%$ decrease in ROS levels compared to control), and down-regulated the production of lipopolysaccharide (LPS)-stimulated inflammatory markers $(22.1,19.9,74.8$, and 87.6\% decrease in NOS, IL-6, PGE 2 , and TNF $\alpha$ levels, respectively, compared to control) in murine BV-2 microglial cells. Moreover, in a non-contact co-culture cell model, differentiated human SH-SY5Y neuronal cells were exposed to conditioned media from BV-2 cells treated with MSX (100 $\mu \mathrm{g} / \mathrm{mL})$ and LPS or LPS alone. MSX-BV-2 media increased SH-SY5Y cell viability by 13.8\% compared to media collected from LPS-BV-2 treated cells. Also, MSX ( $10 \mu \mathrm{g} / \mathrm{mL}$ ) showed protective effects against $A \beta_{1-42}$ induced neurotoxicity and paralysis in Caenorhabditis elegans in vivo. These data support the potential neuroprotective effects of MSX warranting further studies on this natural product.

Keywords Maple syrup phenolics; neuroprotective; neuroinflammation; amyloid $\beta_{1-42}$; Caenorhabditis elegans; Alzheimer's disease 


\section{Introduction}

Microglia cells are resident immune cells in the central nervous system which can be activated by abnormalities including oxidative and inflammatory stresses [1]. Chronic activation of microglia cells leads to the production of pro-inflammatory cytokines including nitric oxide synthase (NOS), interleukins, prostaglandins (PGs), and tumor necrosis factor $\alpha$ (TNF $\alpha)$ [2]. These neurotoxic inflammatory factors result in massive neuronal cell death and are linked to several neurodegenerative diseases including Alzheimer's disease (AD), a progressive neurodegenerative disease [3]. AD is the most common cause of dementia and will afflict $11 \%$ of the United States population over the age of 65 costing the nation \$226 billion in 2015 alone [4]. It is estimated that by 2050 , the worldwide incidence of AD will triple unless innovative strategies, including dietary intervention, are implemented in addition to lifestyle changes and chronic disease management [5].

The extensive neuronal loss that leads to functional and memory impairments in AD patients has been attributed to the proteotoxicity of extracellular amyloid-beta $(A \beta)$ plaques and intracellular tau neurofibrillary tangles $[6,7]$. Several pharmacological therapies focus on interrupting the underlying pathogenesis of $A D$ in the brain [8]. While a common target is $A \beta$ plaques, these approaches have largely been unsuccessful due to limitations in current scientific understanding of all of the etiological and pathophysiological factors relevant in AD. 
Therefore, there is a critical need to discover new therapeutic approaches to manage AD, especially since several drugs currently in clinical evaluation have been associated with severe side effects in patients [9]. Consequently, similar to several other chronic human diseases, the integration of natural products as part of a non-toxic dietary intervention strategy holds great promise for AD prevention [10]. This is due, in part, to the multi-mechanistic modes of action exerted by natural compounds, whereby, they could potentially prevent and/or delay the deleterious effects of oxidative stress, neuroinflammation, proteotoxicity, and other factors implicated in the pathogenesis of neurodegenerative diseases.

Published data suggest that several phenolic-rich plant foods including certain fruits, berries, spices, nuts, green tea, and olive oil may show promise for AD prevention [10]. Phenolic compounds include a chemically diverse group of secondary metabolites containing a phenolic moiety which are prevalent in plant foods and their derived products. Indeed, dietary plant extracts and their purified phenolic constituents such as resveratrol (from grape and red wine) [10], anthocyanins (from berries) [11], curcumin (from turmeric spice) [12], oleocanthal (from olive oil) [13], and catechin (from grape seed) [14], have been studied extensively for their biological effects against AD. Several studies have noted that the biological activities of these single purified molecules are potentiated by synergistic, additive, and/or complementary effects of multiple (minor) constituents present in their respective phenolic-enriched 'whole' plant extracts [15]. 
Thus, chemically standardized botanical extracts, delivered in food, supplement, and pharmaceutical matrices, hold great promise for the prevention of neurodegenerative diseases in future human clinical studies.

Maple syrup is a plant-derived natural sweetener obtained by boiling the sap collected primarily from the sugar maple (Acer saccharum) species. Apart from its predominant constituent which is sucrose, maple syrup also contains oligosaccharides, amino acids, organic acids, vitamins, minerals, and phytohormones [16]. In addition, a large number of natural phenolic compounds originally present in the xylem sap of the sugar maple plant survive the boiling process and persist in maple syrup [16-19]. However, despite the wide consumption of maple syrup worldwide, and knowledge that it contains a diverse array of phenolic constituents (over fifty phenolics belonging to lignan, stilbene, flavonoid, coumarin, and phenolic acid subclasses have been isolated and identified from maple syrup) [17-19, 22], studies evaluating their health promoting effects are very limited. Moreover, based on our knowledge to date, few studies have been conducted to evaluate the neuroprotective effects of this natural sweetener $[20,21]$. In one of those studies, a phenolic-enriched maple syrup extract was shown to decrease oligomerization and aggregation of both $A \beta_{1-42}$ and tau peptides, which are proteins involved in AD pathogenesis [20]. In light of the aforementioned factors, and given the paucity of data on the neuroprotective effects of natural plant-derived sweeteners, including maple syrup, we designed the current study.

Our group has previously reported on the chemical standardization of a food grade phenolic-enriched maple syrup extract (named MSX) for nutraceutical applications [22]. This extract contains the natural phenolic constituents, as well as other substances (amino acids, organic acids, minerals, and oligosaccharides) present in the whole maple syrup food but with reduced sucrose content. MSX is well tolerated and its safety has been established in animal 
studies (in Sprague-Dawley rats) [22]. Notably, MSX shows anti-inflammatory effects (based on in vitro studies in RAW264.7 macrophages) superior to any of its purified phenolic constituents alone [23]. Therefore, given the aforementioned factors about the additive and synergistic effects observed with 'whole' plant extracts [15], as well as the possibility of a nutraceutical/botanical supplement being utilized as a dietary intervention strategy in future human clinical studies, we have chosen to advance our studies on this natural product by evaluating MSX rather than any of its purified single constituents alone.

Herein, we sought to evaluate the neuroprotective effects of MSX against AD using a combination of biophysical (Thioflavin T assay, transmission electron microscopy, circular dichroism, dynamic light scattering, and zeta potential), in vitro (in murine BV-2 microglial, and human SY-SY5Y neuronal cells) and in vivo (in Caenorhabditis elegans) studies. This is the first study to evaluate the effects of maple syrup constituents on markers of oxidative and inflammatory stresses in brain cells and its potential to delay $A \beta_{1-42}$ induced neurotoxicity and paralysis in an in vivo $A D$ model.

\section{Materials and Methods}

\section{Chemicals}

Human amyloid peptide 1-42 $\left(A \beta_{1-42}\right)$ was purchased from AnaSpec (Fremont, $C A$, USA).

Resveratrol (RESV), Folin-Ciocalteu reagent, thioflavin T (ThT), 2', $7^{\prime}$-dichlorofluorescin diacetate (DCFDA), lipopolysaccharide (LPS) and solvents were purchased from Sigma-Aldrich (St. Louis, MO, USA). Uranyl acetate was purchased from Structure Probe Inc. (West Chester, PA, USA). ELISA kits for prostaglandin E2 (PGE $)$, and tumor necrosis factor $\alpha$ (TNF $\alpha)$ and interleukin-6 (IL6) were purchased from Cayman Chemical (Ann Arbor, MI, USA), and Abcam (Cambridge, MA, USA), respectively. 


\section{Phenolic-enriched maple syrup extract (MSX)}

The phenolic-enriched maple syrup extract (MSX), was prepared according to our previously reported methods $[19,22,23]$. The details of the MSX preparation and its chemical characterization (by HPLC-DAD analyses by comparison to authentic phenolic standards previously isolated from maple syrup), the structures of the compounds identified therein, and the carbohydrate (sucrose, glucose and fructose) and phenolic (by Folin-Ciocalteu assay based on gallic acid equivalents, GAEs) contents, are provided in the Supplementary Materials.

\section{$A \beta_{1-42}$ thioflavin $T(T h T)$ binding assay}

$A \beta_{1-42}$ solutions were prepared following previously reported methods with minor modifications [24]. In brief, fibril formation was induced by incubating $50 \mu \mathrm{M} \mathrm{A} \beta_{1-42}$ solutions at $37^{\circ} \mathrm{C}$ for $5 \mathrm{~d}$. Treatment included MSX at varying concentrations $(50,100,200$ and $500 \mu \mathrm{g} / \mathrm{mL})$ and the widely studied neuroprotective polyphenolic compound, resveratrol (RESV), used as the positive control (at $50 \mu \mathrm{g} / \mathrm{mL}$ ). The ThT binding assay was used to evaluate $A \beta_{1-42}$ fibrillation by adding ThT solution $(200 \mu \mathrm{L})$ at a concentration of $10 \mu \mathrm{M}$ to each sample $(50 \mu \mathrm{L})$. The intrinsic fluorescence was immediately measured using a Spectra Max M2 spectrometer (Molecular Devices, Sunnyvale, CA, USA) at excitation and emission wavelengths of 450 and $483 \mathrm{~nm}$, respectively. Percent inhibition (\% inhibition) of each treatment was calculated based on arbitrary fluorescence (FU) by the following equation: \% inhibition $=[(F U$ of control $-F U$ of treatment)/FU of control] $\times 100 \%$. 


\section{Transmission electron microscope (TEM) analyses}

$A \beta_{1-42}$ solutions for TEM analysis were prepared under the same conditions as the ThT binding assay. $A \beta_{1-42}$ fibril formation was visualized by TEM as previously reported $[25,26]$. The blank, control, and MSX (500 $\mu \mathrm{g} / \mathrm{mL}$ ) treated samples (5 $\mathrm{LL}$ each) were placed onto a 200 mesh carboncoated copper TEM grid (Ted Pella Inc., Redding, CA, USA) for 2 min. The excess fluid was removed by filter paper and $5 \%$ uranyl acetate $(10 \mu \mathrm{L})$ was placed onto the grid for $2 \mathrm{~min}$. $A \beta_{1-42}$ fibril formation was imaged using a Joel Corp. (Peabody, MA, USA) JEM 2100 TEM with a LaB6 illumination filament operating at $200 \mathrm{kV}$.

\section{Circular dichroism (CD) analyses}

The change in the secondary structure of $A \beta$ solutions were measured using a Jasco J-1100 CD spectropolarimeter (Jasco, Tokyo, Japan). Analyzed samples included $50 \mu \mathrm{M}$ native $A \beta_{1-42}$ solution (non-incubated) and the aforementioned fibril-enriched $A \beta$ solutions. $C D$ measurements were carried out in the far-ultraviolet region from 190-260 nm using a quartz cuvette with a path length of $0.1 \mathrm{~cm}$. The bandwidth was set at $1 \mathrm{~nm}$ for each run and 5 repetitive scans were obtained. The Selcon 3 method was applied to calculate the secondary structure composition using CDPro software (Jasco, Tokyo, Japan). 


\section{Dynamic light scattering (DLS) and zeta potential measurement}

The size of $A \beta_{1-42}$ aggregates and zeta potential were measured with a Zetasizer Nano-ZS90 (Malvern, Worcestershire, UK) as previously reported for the analyses of amyloid fibril formation [27]. Test samples included: 1) $50 \mu \mathrm{M}$ freshly prepared $A \beta_{1-42}$, 2) $50 \mu \mathrm{M} A \beta_{1-42}$ aggregated at $37^{\circ} \mathrm{C}$ for $5 \mathrm{~d}$ and, 3) $50 \mu \mathrm{M}$ aggregated $A \beta_{1-42}$ co-treated with $\mathrm{MSX}(500 \mu \mathrm{g} / \mathrm{mL})$ for $5 \mathrm{~d}$. Each measurement represents the average of three independent experiments and 100 consecutive runs were obtained for each sample at an operating temperature of $25^{\circ} \mathrm{C}$.

\section{Cell culture}

The BV-2 murine microglial cells were a gift from Dr. Grace Y. Sun (University of Missouri at Columbia, MO, USA) and the SH-SY5Y (human neuroblastoma) cells were obtained from the American Type Culture Collection (ATCC). Cell lines were maintained using high glucose $(4.5 \mathrm{~g} / \mathrm{L})$ DMEM/F12 (Life Technologies, Gaithersburg, MD, USA) supplemented with $10 \%$ heat inactivated FBS (Life Technologies, Gaithersburg, MD, USA), 1\% P/S (100 U/ml penicillin, 100 $\mu \mathrm{g} / \mathrm{ml}$ streptomycin) (Life Technologies, Gaithersburg, MD, USA) and incubated in $5 \% \mathrm{CO}_{2}$ at $37^{\circ} \mathrm{C}$. After reaching a confluency of $85 \%$, the SH-SY5Y cells were differentiated for $7 \mathrm{~d}$ using alltrans retinoic acid $(10 \mu \mathrm{M})$ while changing media every $48 \mathrm{~h}$ to ensure proper neurite growth and cell morphology. All samples were dissolved in DMSO (Sigma Aldrich, St. Louis, MO, USA) to yield a $10 \mathrm{mg} / \mathrm{mL}$ stock solution. Samples were further diluted to yield solutions with a final DMSO concentration of less than $0.1 \%$. 


\section{Measurement of cell viabilities}

Cell viabilities of BV-2 and SH-SY5Y cells were evaluated using the MTT [3-(4,5-dimethylthiazol-2yl)-2,5-diphenyltetrazolium bromide] colorimetric assay [28] and CellTiter-Glo 2.0 (CTG 2.0) one step assay (Promega Fitchburg, WI, USA), respectively. Briefly, in the MTT assay, BV-2 cells were seeded into 48 -well plates at a density of $10^{4}$ cells/well in DMEM. After $24 \mathrm{~h}$, the cells were treated with MSX (50 or $100 \mu \mathrm{g} / \mathrm{mL}$ ) for $48 \mathrm{~h}$. At the end of the treatment, the cell viability was measured by using cell-counting kit 8 reagent (Dojindo Molecular Technologies, Rockville, MD, USA), according to the manufacturer's instructions. In the CTG 2.0 assay, SH-SY5Y cells were seeded at $10^{5}$ cells $/ \mathrm{mL}$ to yield $80-85 \%$ confluency in a standard white walled clear bottom 96 -

well plate after $24 \mathrm{~h}$ initial incubation. Following a secondary incubation period ( $24 \mathrm{~h}$ ), CTG 2.0 was added in a 1:1 ratio with the existing media in the wells and mixed for 2 min on an orbital shaker prior to luminescence measurement using the Spectramax M2 (Molecular Devices Sunyvale, CA, USA).

\section{Measurement of levels of reactive oxygen species (ROS)}

BV-2 cells were seeded into 48 -well plates at a density of $10^{4}$ cells/well in DMEM. After $24 \mathrm{~h}$, the cells were pretreated with MSX (50 or $100 \mu \mathrm{g} / \mathrm{mL}$ ) or the positive control, resveratrol (RESV; 5 $\mu \mathrm{g} / \mathrm{mL}$ ) for $24 \mathrm{~h}$. Then, the medium was replaced with DMEM containing $20 \mu \mathrm{M}$ DCFDA for 20 min. The cells were washed with phosphate buffered saline and incubated with $\mathrm{H}_{2} \mathrm{O}_{2}$ at a nontoxic concentration $(3.0 \mu \mathrm{g} / \mathrm{mL})$ for $1 \mathrm{~h}$. The fluorescence signal was read at excitation and emission wavelengths of 485 and $525 \mathrm{~nm}$, respectively, using a Spectra Max M2 spectrometer (Molecular Devices, Sunnyvale, CA, USA). 


\section{Measurement of nitric oxide species (NOS) levels by Griess assay}

BV-2 microglial cells in 24-well plates $(n=4)$ at $85 \%$ confluency $\left(10^{5}\right.$ cells/well) were serumstarved for $4 \mathrm{~h}$ prior to the treatments. Then the cells were incubated with MSX (50 or 100 $\mu \mathrm{g} / \mathrm{mL}$ ) or the positive control, resveratrol (RESV; $5 \mu \mathrm{g} / \mathrm{mL}$ ) for $1 \mathrm{~h}$ before lipopolysaccharide (LPS) was added. After $23 \mathrm{~h}$ incubation, the cell culture media were collected and centrifuged. The supernatants were measured for total nitric oxide (NO) levels using the Griess assay kit (Promega, Fitchburg, WI, USA) as previously reported [23]. Sodium nitrite, at different dilutions, was used to generate a standard curve which was used to calculate nitrite concentrations.

\section{Measurements of IL-6, PGE 2 , and TNF $\alpha$ levels}

Supernatants collected from the NO experiments above were collected and assayed for inflammatory cytokines including interleukin-6 (IL-6), prostaglandin E2 $\left(\mathrm{PGE}_{2}\right)$ and tumor necrosis factor $\alpha$ (TNF $\alpha$ ). Quantification of $\mathrm{PGE}_{2}$ and TNF $\alpha$ were measured using enzyme linked immunosorbent assay (ELISA) kits based on the respective manufacturer protocols.

\section{Non-contact co-culture model with BV-2 and SH-SY5Y cells}

BV-2 and SH-SY5Y cells were co-cultured as previously reported [29-31]. Briefly, BV-2 and differentiated SH-SY5Y cells (both cell lines at a density of $10^{5}$ cells $/ \mathrm{mL}$ ) were plated in 12-well and 96-well plates, respectively, and allowed to attach for $24 \mathrm{~h}$. 
The BV-2 cells were then exposed to MSX (50 and $100 \mu \mathrm{g} / \mathrm{mL}$ ) or RESV $(5 \mu \mathrm{g} / \mathrm{mL}$; positive control) for $1 \mathrm{~h}$ prior to exposure to LPS $(1 \mu \mathrm{g} / \mathrm{mL})$ for a duration of $24 \mathrm{~h}$ of treatment. Conditioned media were then collected from the BV-2 cells and centrifuged at $18,000 \mathrm{rcf}$ for 5 $\min$ at $4^{\circ} \mathrm{C}$. The media from the SH-SY5Y cells was removed and replaced with the centrifuged conditioned media from the BV-2 cells and left to incubate for $24 \mathrm{~h}$ after which they were subjected to the CTG 2.0 assay to assess cell viability.

\section{Transgenic Caenorhabditis elegans in vivo assay}

The C. elegans nematode assay was carried out as previously reported by our group [24]. Briefly, the transgenic $C$. elegans strain CL4176, developed to express human amyloid $\beta_{1-42}$ in the muscle tissue in response to heat shock, were obtained from the Caenorhabditis Genetics Center (CGC) (University of Minnesota, Minneapolis, MN, USA). Worms were grown and maintained at $16{ }^{\circ} \mathrm{C}$ on $60 \mathrm{~mm}$ culture plates with Nematode Growth Medium (NGM). Media was poured aseptically into culture plates ( $10 \mathrm{~mL}$ for $60 \mathrm{~mm}$ ) using a peristaltic pump and allowed to solidify for $36 \mathrm{~h}$. NGM culture plates were then inoculated with $50 \mu \mathrm{L}$ of Escherichia coli OP50 (CGC, University of Minnesota, Minneapolis, MN, USA) overnight cultures and incubated for $8 \mathrm{~h}$ at $37^{\circ} \mathrm{C}$. The $\mathrm{C}$. elegans were maintained by picking 2-3 young adult worms onto freshly inoculated NGM plates every 4-7 d. MSX was dissolved in S-basal buffer and methanol solution $(1: 1 ; \mathrm{v} / \mathrm{v})$ and further diluted in S-basal buffer to a yield a final concentration of $1 \mathrm{mg} / \mathrm{mL}$ which served as the stock solution. The MSX stock solution was added directly to the NGM media for a final treatment concentration of $10 \mu \mathrm{g} / \mathrm{mL}$. 
Upon development of the eggs to the L3 larval stage, the incubation temperature of the plates was increased from 16 to $25^{\circ} \mathrm{C}$ to induce the expression of $A \beta_{1-42}$. Mobility scoring was conducted beginning at $20 \mathrm{~h}$ after the temperature increase and continued in $2 \mathrm{~h}$ increments until all of the worms were paralyzed. Failure to respond to touch (prodding with a worm pick) and, absence of pharyngeal pumping, were used to score paralyzed/dead worms.

\section{Statistical analyses}

Data are presented as mean \pm standard deviation of at least 3 separate experiments. For the ThT assay, statistical analysis was conducted by one-way factorial ANOVA with Tukey-Kramer post hoc comparisons. Cellular assay data were expressed as means \pm standard errors. $P$ values were generated using ANOVA followed by Dunnett's test for multiple comparisons of group means. For the in vivo C. elegans assay, the Kaplan-Meier method was used to compare the lifespan survival curves and the survival differences were tested for statistical significance using the Log rank test (Mantel Cox). Significance for all tests was defined as: $p \leq 0.05\left({ }^{*}\right), p \leq 0.01\left({ }^{* *}\right), p \leq 0.001$ $(* * *)$, and $\mathrm{p} \leq 0.0001(* * * *)$. GraphPad Prism software 6.0 (GraphPad Software, Inc., San Diego, CA) was used for all statistical analysis calculations. 


\section{Results and Discussion}

\section{Chemical characterization of phenolic-enriched maple syrup extract (MSX)}

MSX is a chemically standardized food grade phenolic-enriched maple syrup extract, previously developed by our group for nutraceutical applications, with established safety and tolerability data in animals [22]. For the present study, MSX was prepared in our laboratory based on methods previously reported by our group (details provided in Supplementary Materials).

Our group has previously isolated and identified (using NMR and mass spectrometry) over fifty phenolic compounds from maple syrup $[19,22,23]$. Therefore, through comparison of HPLC-DAD retention times with these chemical marker standards, as shown in Fig. 1, a total of thirty-seven compounds (chemical structures provided in Fig. S2 in the Supplementary Materials) were identified in MSX. As expected, the majority of the compounds in MSX are phenolic compounds, belonging to lignan, coumarin, phenolic acid, and stilbene sub-classes.

The phenolic content of MSX used in the present study was determined as $24.0 \%$ (based on gallic acid equivalents, GAEs). In addition, it had a sucrose content of ca. $10.0 \%$ as compared to pure maple syrup which was $63 \%$. The levels of fructose and glucose in MSX were $<0.1 \%$. We next sought to evaluate the neuroprotective effects of MSX using a combination of biophysical (Thioflavin T assay, transmission electron microscopy, circular dichroism, dynamic light scattering and zeta potential), in vitro (murine BV-2 microglial, and human SH-SY5Y cells) and in vivo (Caenorhabditis elegans) studies (further described below). 


\section{MSX inhibits $A \beta$ fibrillation}

$A \beta$ deposition in brain leads to the formation of senile plaques and causes neurotoxicity in $A D$ patients. During the progression of $A D$, soluble unstructured $A \beta$ peptides gradually reassemble to insoluble $A \beta$ fibrils, a structure enriched in $\beta$-sheet confirmation. Therefore, MSX was evaluated for its inhibitory effects on the formation of $A \beta_{1-42}$ fibril structures using a combination of biophysical methods. $A \beta_{1-42}$ fibrillation was first quantified by the fluorescence based ThT binding assay (Fig. 2A) and the morphology of the fibrils was visualized by TEM (Fig. 2B). The $\beta$ sheet contents were measured by circular dichroism (CD) studies (Fig. 3). The size and zeta potential of $A \beta_{1-42}$ aggregates were measured by zetasizer (Table 1 ).

As shown in Fig. 2A, the control generated the highest binding level in the ThT assay indicating that the control group had the highest level of $A \beta_{1-42}$ fibrillation. The $A \beta_{1-42}$ fibrillation level significantly decreased in all of the treatment samples including the different concentrations of MSX (from $50-500 \mu \mathrm{g} / \mathrm{mL}$ ) and the neuroprotective polyphenolic compound, resveratrol (RESV), used as a positive control. 
The data generated in the current study for RESV $(50 \mu \mathrm{g} / \mathrm{mL}$, inhibition $=77.3 \%, \mathrm{p} \leq 0.001)$ is in agreement with previously published data [32]. Similarly, the MSX treated solutions showed a concentration-dependent effect at 500, 250, 100 and $50 \mu \mathrm{g} / \mathrm{mL}$, reducing $A \beta$ fibrillation by 63.5 , 55.9, 54.9 and $18.9 \%$, respectively. Therefore, the ThT assay data suggested that both MSX and RESV significantly inhibited A $\beta$ fibrillation. Also, it should be noted that the lowest test concentration of MSX (50 $\mathrm{gg} / \mathrm{mL})$ significantly inhibited the formation of $A \beta_{1-42}$ fibrils $(p \leq 0.05)$.

Our findings from the ThT assay were further confirmed by the TEM analyses. As shown in Fig. $2 B$, the control $A \beta_{1-42}$ sample generated morphology of aggregates typically seen in the fibrillation process of $A \beta_{1-42}[25,26]$. In contrast, the $A \beta_{1-42}$ co-treated with $M S X(500 \mu \mathrm{g} / \mathrm{mL})$ sample showed a different morphology compared to the control. The overall fibrils in the MSX treated solution were significantly less dense but more amorphous, indicating that the formation of highly ordered $A \beta$ fibrils was reduced by MSX.

Next, we measured the $\beta$-sheet contents in $A \beta_{1-42}$ solutions using $C D$ spectroscopy (shown in Fig. 3 and Table 1). Compared to native non-aggregated $A \beta_{1-42}$, all of the incubated samples displayed a signature dip at $220 \mathrm{~nm}$, indicating the formation of $\beta$-sheets structures [33]. However, in the MSX treated sample $(500 \mu \mathrm{g} / \mathrm{mL})$, the conformational changes of the $\beta$ sheets were $10 \%$ lower than that of control ( 49.6 vs. $59.6 \%)$. A similar trend was observed for the positive control, RESV $(50 \mu \mathrm{g} / \mathrm{mL})$, which showed a $\beta$-sheet content of $46.7 \%$. 
$A \beta_{1-42}$ fibrillation can cause the formation of aggregates which can be measured by dynamic light scattering (DLS) [27]. Based on DLS measurements (shown in Table 1), no measurable aggregates were observed in native freshly prepared $A \beta_{1-42}$. In contrast, after incubation at $37^{\circ} \mathrm{C}, A \beta_{1-42}$ yielded aggregates with particle size of $317.9 \mathrm{~nm}$ and a dominant content of $86.4 \%$ which were reduced to $39.84 \mathrm{~nm}$ and $77 \%$, respectively, by MSX ( $500 \mu \mathrm{g} / \mathrm{mL}$ ). As previously reported, the zeta potential of $A \beta_{1-42}$ shifts to a more negative value as aggregation progresses [27]. Our data is in agreement with these observations [27] wherein we observed a significant decrease in zeta potential values from $-4.58 \mathrm{mV}$ for freshly prepared $A \beta_{1-42}$ to -22.4 $m V$ for aggregated $A \beta_{1-42}$. However, the MSX treated $A \beta_{1-42}$, generated an increase in zeta potential value of $-17.6 \mathrm{mV}$, indicating that the aggregation process was less extensive than the control.

Taken together, the biophysical data from the ThT assay, TEM analyses, CD experiment, DLS, and zeta potential measurements suggested that the MSX treatments effectively inhibited $A \beta_{1-42}$ structural transformation from soluble non-toxic peptides to insoluble neuro-toxic fibrils. These data are in agreement with the previous report for a phenolic-enriched maple syrup extract [20]. However, to date, no previous studies have been conducted to evaluate the neuroprotective effects of maple syrup in brain cells or in an in vivo AD model; therefore, we proceeded with the following experiments (described below). 


\section{MSX protects murine BV-2 microglia against $\mathrm{H}_{2} \mathrm{O}_{2}$-induced oxidative stresses in vitro}

Microglia cells are resident innate immune cells in the central nervous system with a similar functional role as macrophages. An important cellular response in neurodegenerative diseases pathogenesis is microglial activation. Moreover, the over-activation of microglia can lead to the release of a variety of pro-inflammatory mediators which are potentially neurotoxic. This activation process is often promoted by cellular oxidative stress factors including reactive oxygen species (ROS) and thus, the reduction of ROS levels alleviates microglial activation and related neuronal disorders [34]. Therefore, MSX was evaluated (at non-toxic concentrations, ranging from 10 to $100 \mu \mathrm{g} / \mathrm{mL}$ based on the MTT assay, as shown in Fig. 4A) for its effects on $\mathrm{H}_{2} \mathrm{O}_{2}(3.0 \mu \mathrm{g} / \mathrm{mL})$ induced ROS production in murine BV-2 microglia cells. As shown in Fig. 4B, MSX decreased $\mathrm{H}_{2} \mathrm{O}_{2}$-induced ROS production in BV-2 cells. At concentrations of 50 and 100 $\mu \mathrm{g} / \mathrm{mL}, \mathrm{MSX}$ reduced $\mathrm{H}_{2} \mathrm{O}_{2}$-induced ROS production by 9.9 and $16.1 \%$, respectively. The positive control, RESV, decreased $\mathrm{H}_{2} \mathrm{O}_{2}$-induced ROS levels by $66.2 \%$ at a concentration of $5 \mu \mathrm{g} / \mathrm{mL}$, which was similar to previously reported data [34]. 


\section{MSX protects murine BV-2 microglia against LPS-induced inflammatory stresses in vitro}

Our group has previously shown that MSX reduced pro-inflammatory cytokines in RAW264.7

murine macrophages [23] but to date, these effects have not been investigated in brain cells. Herein, we evaluated whether MSX could reduce levels of biomarkers for neuronal inflammation, induced by LPS, in BV-2 cells. Indeed, the LPS treated cells produced higher NO levels compared to untreated cells (51.9 vs $9.2 \mu \mathrm{M})$. As shown in Fig. 5A, MSX inhibited NO production. At 50 and $100 \mu \mathrm{g} / \mathrm{mL}, \mathrm{MSX}$ decreased NO production by 10.0 and $22.1 \%$, respectively. Similar to previously reported data [34], RESV (5 $\mu \mathrm{g} / \mathrm{mL})$, reduced LPS-induced NO production by $23 \%$.

Next, MSX was further evaluated for its effects on inflammatory cytokines, IL-6, PGE 2 and TNF $\alpha$, in the BV-2 cells. As shown in Fig. 2A, after incubation of the BV-2 microglial cells with LPS, MSX (100 $\mu \mathrm{g} / \mathrm{mL}$ ) significantly (by 19.9\%) reduced IL-6 levels as compared to the LPS treated control cells. However, the IL-6 production was not significantly (4.2\%) reduced by treatment of MSX at the lower concentration of $50 \mu \mathrm{g} / \mathrm{mL}$. The levels of $\mathrm{PGE}_{2}$ were reduced by 52.9 and $74.8 \%$, after treatment with 50 and $100 \mu \mathrm{g} / \mathrm{mL}$ MSX, respectively, compared to LPS treated control cells, In comparison, the positive control, RESV $(5 \mu \mathrm{g} / \mathrm{mL})$, reduced $\mathrm{PGE}_{2}$ levels by $22.5 \%$ which was similar to previously reported data [34] (Fig. 5C). Similarly, the levels of TNFa were down-regulated by treatment of BV-2 cells with MSX (Fig. 5D) by 75.2 and $87.6 \%$, after treatment with 50 and $100 \mu \mathrm{g} / \mathrm{mL}$ MSX, respectively, compared to the LPS treated cells. The positive control, RESV $(5 \mu \mathrm{g} / \mathrm{mL})$, decreased TNF $\alpha$ levels by $31.4 \%$ similar to previously reported data [34]. 


\section{MSX reduces cytotoxicity of human SH-SY5Y neuronal cells after exposure to murine BV-2 microglia conditioned media}

Co-culture systems have been employed to investigate the impact of microglia and its inflammatory mediators on the cell death of neurons [29-31, 35]. Therefore, we used a noncontact cell culture model (using conditioned media) to evaluate the protective effects of MSX against neurotoxicity (induced by inflammatory biomarkers released by LPS-stimulated BV-2 microglia) of differentiated human SH-SY5Y neuronal cells as previously reported [30, 31]. As shown in Fig. 6, media from BV-2 cells (treated with LPS alone) significantly decreased (15.0\%) the cell viability of differentiated SH-SY5Y neuronal cells. On the contrary, the conditioned media from BV-2 cells treated with MSX (at 100 and $50 \mu \mathrm{g} / \mathrm{mL}$ ) reduced SH-SY5Y neuronal cell death by 17.7 and $13.8 \%$, respectively. The positive control, RESV $(5 \mu \mathrm{g} / \mathrm{mL})$, decreased the SHSY5Y neuronal cell death by $30.5 \%$. This observation indicated that MSX was able to ameliorate microglia-mediated neuronal cell death possibly by decreasing the production of inflammatory biomarkers by the microglia. 


\section{MSX protects $A \beta_{1-42}$ induced neurotoxicity and paralysis in Caenorhabditis elegans in vivo}

We next evaluated the effects of MSX in an established $C$. elegans in vivo model of AD [24]. The mobility curves for the $C$. elegans CL4176 strain after the $A \beta_{1-42}$ induction of muscular paralysis at $25^{\circ} \mathrm{C}$ are shown in Fig. 6 . Compared to the control worms (Fig. 7A), treatment with $10 \mu \mathrm{g} / \mathrm{mL}$ MSX did not have any significant effect on the median survival/mobility in C. elegans post induction of $A \beta_{1-42}$ induced neurotoxicity and paralysis. However, MSX treatment (Fig. 7B) significantly increased the maximum survival/mobility by $3.0 \%$ and decreased the mean survival/mobility post induction of $A \beta_{1-42}$ induced neurotoxicity and paralysis by $10.4 \%$ (as shown in Table 2).

Mitochondrial dysfunction, inflammation, oxidative stress, and activation of apoptotic signaling due to intra- and extracellular accumulation of misfolded or damaged proteins has been linked to the etiopathology of neurodegenerative diseases, including $A D[36,37]$.

Therefore, therapies that specifically target mediators of proteotoxicity and inflammation may be more successful in ameliorating neurodegenerative processes [38]. The transgenic AD model of $C$. elegans used in our study specifically measures the intracellular aggregation of the $A \beta_{1-42}$ peptide in muscle tissues and permits investigation of inflammatory and proteotoxicity contributors of $A \beta_{1-42}$ peptide aggregation and subsequent neuromuscular paralysis $[24,39,40]$. In response to treatment with $M S X$, the heat-shock induced aggregation of $A \beta_{1-42}$ peptide and resultant paralysis was significantly delayed in the $C$. elegans model. 
These results corroborated our observations from the biophysical and in vitro studies wherein MSX reduced $A \beta_{1-42}$ fibrillation, oxidative stress, and inflammatory cytokines. It is possible that the reduced proteotoxicity in C. elegans in response to MSX could be due to the presence of certain bioactive compounds that interacts with amyloidogenic proteins to prevent their oligomerization (and thus aggregation) [41]. However further studies would be required to confirm this. Overall, the current study with MSX support studies done with other natural products including coffee bean extract [42], Ginkgo biloba [41], curcumin, and several tropical fruit extracts $[39,40]$, suggesting similar mechanism/s for reducing $A \beta_{1-42}$ aggregation by abrogating proteotoxicity and inflammation. 


\section{Conclusion}

In summary, using a combination of biophysical, in vitro, and in vivo studies, a phenolicenriched extract of maple syrup (MSX) was shown to reduce $A \beta_{1-42}$ fibrillation and decrease oxidative and inflammatory stresses in murine BV-2 microglial cells and SHSY-5Y neuronal cells. Furthermore, MSX imparted protective effects on $A \beta_{1-42}$ aggregation induced neurotoxicity and

paralysis in C. elegans. However, further in vivo studies to confirm the neuroprotective effects of MSX, and to predict a human equivalent consumption dose, are necessary given that the current data does not account for important physiological considerations such as bioavailability and metabolism. Nevertheless, these findings add to recently published data supporting the potential neuroprotective effects of maple syrup [20,21], warranting further studies on this natural plant-derived sweetener. 


\section{Conflict of interest statement}

The authors have declared no conflicts of interest.

\section{Acknowledgment}

The spectroscopic data were acquired from instruments located in the RI-INBRE core facility supported by Grant \# P20GM103430 from the National Institute of General Medical Sciences of the National Institutes of Health. We thank Dr. Grace Y. Sun (University of Missouri at Columbia, MO, USA) for kindly providing the BV-2 murine microglial cells and Dr. Richard Kingsley and the URI TEM facility (URI Chemical Engineering) for assisting with TEM sample imaging.

\section{Supplementary Materials}

Supplementary data associated with this article can be found in the online version. 


\section{REFERENCES}

1. Praticò D, Trojanowski JQ (2000) Inflammatory hypotheses: novel mechanisms of Alzheimer's neurodegeneration and new therapeutic targets? Neurobiol Aging 21: 451443

2. Block ML, Zecca L, Hong J-S (2007) Microglia-mediated neurotoxicity: uncovering the molecular mechanisms. Nat Rev Neurosci 8:57-69

3. Heneka MT, O'Banion MK (2007) Inflammatory processes in Alzheimer's disease. J Neuroimmunol 184:69-91

4. Alzheimer's Association (2013) 2013 Alzheimer's disease facts and figures. Alzheimer's \& Dementia 9:208-245

5. Sloane PD, Zimmerman S, Suchindran C, Reed P, Wang L, Boustani M, Sudha S (2002) The public health impact of Alzheimer's disease, 2000-2050: potential implication of treatment advances. Annu Rev Public Health 23:213-231

6. Hardy JA, Higgins GA (1992) Alzheimer's disease: the amyloid cascade hypothesis. Science 256:184-185

7. Haass C, Selkoe DJ (1993) Cellular processing of $\beta$-amyloid precursor protein and the genesis of amyloid $\beta$-peptide. Cell 75:1039-1042

8. Herrmann N, Chau SA, Kircanski I, Lanctôt KL (2011) Current and emerging drug treatment options for Alzheimer's disease. Drugs 71:2031-2065

9. Vellas B, Pesce A, Robert PH, Aisen PS, Ancoli-Israel S, Andrieu S, Cedarbaum J, Dubois B, Siemers E, Spire J-P (2011) AMPA workshop on challenges faced by investigators conducting Alzheimer's disease clinical trials. Alzheimer's \& Dementia 7:e109-e117

10. Kim J, Lee HJ, Lee KW (2010) Naturally occurring phytochemicals for the prevention of Alzheimer's disease. J Neurochem 112:1415-1430

11. Willis LM, Shukitt-Hale B, Joseph JA (2009) Recent advances in berry supplementation and age-related cognitive decline. Curr Opin Clin Nutr Metab Care 12:91-94

12. Ringman JM, Frautschy SA, Cole GM, Masterman DL, Cummings JL (2005) A potential role of the curry spice curcumin in Alzheimer's disease. Curr Alzheimer Res 2:131-136

13. Abuznait AH, Qosa H, Busnena BA, El Sayed KA, Kaddoumi A (2013) Olive-oil-derived oleocanthal enhances $\beta$-amyloid clearance as a potential neuroprotective mechanism against Alzheimer's disease: In vitro and in vivo studies. ACS Cheml Neurosci 4:973-982

14. Wang J, Ho L, Zhao W, Ono K, Rosensweig C, Chen L, Humala N, Teplow DB, Pasinetti GM (2008) Grape-derived polyphenolics prevent $A \beta$ oligomerization and attenuate cognitive deterioration in a mouse model of Alzheimer's disease. J Neurosci 28:6388-6392 
15. Liu RH (2013) Dietary bioactive compounds and their health implications. J Food Sci 78 Suppl 1:A18-25

16. Ball DW (2007) The chemical composition of maple syrup. J Chem Edu 84:1647-1650

17. Abou-Zaid MM, Nozzolillo C, Tonon A, Coppens M, Lombardo DA (2008) Highperformance liquid chromatography characterization and identification of antioxidant polyphenols in maple syrup. Pharm Biol 46:117-125

18. Li L, Seeram NP (2010) Maple syrup phytochemicals include lignans, coumarins, a stilbene, and other previously unreported antioxidant phenolic compounds. J Agric Food Chem 58:11673-11679

19. Li L, Seeram NP (2011) Further investigation into maple syrup yields 3 new lignans, a new phenylpropanoid, and 26 other phytochemicals. J Agric Food Chem 59:7708-7716

20. Hawco CL, Wang Y, Taylor M, Weaver DF (2015) A Maple Syrup extract prevents $\beta$ amyloid aggregation. Can J Neurol Sci:1-4

21. Aaron C, Beaudry G, Parker JA, Therrien M (2016) Maple syrup decreases TDP-43 proteotoxicity in a Caenorhabditis elegans model of amyotrophic lateral sclerosis (ALS). J Agric Food Chem 64:3338-3344

22. Zhang Y, Yuan T, Li L, Nahar P, Slitt A, Seeram NP (2014) Chemical compositional, biological, and safety studies of a novel maple syrup derived extract for nutraceutical applications. J Agric Food Chem 62:6687-6698

23. Nahar PP, Driscoll MV, Li L, Slitt AL, Seeram NP (2014) Phenolic mediated antiinflammatory properties of a maple syrup extract in RAW 264.7 murine macrophages. J Funct Foods 6:126-136

24. Yuan T, Ma H, Liu W, Niesen DB, Shah N, Crews R, Rose KN, Vattem DA, Seeram NP (2016) Pomegranate's neuroprotective effects against Alzheimer's disease are mediated by urolithins, ilts ellagitannin-gut microbial derived metabolites. ACS Chem Neurosci 7:26-33

25. Das S, Stark L, Musgrave IF, Pukala T, Smid SD (2016) Bioactive polyphenol interactions with $\beta$ amyloid: a comparison of binding modelling, effects on fibril and aggregate formation and neuroprotective capacity. Food Funct 7:1138-1146

26. Shoval H, Weiner L, Gazit E, Levy M, Pinchuk I, Lichtenberg D (2008) Polyphenol-induced dissociation of various amyloid fibrils results in a methionine-independent formation of ROS. Biochim Biophys Acta 1784:1570-1577

27. Vetri V, Canale C, Relini A, Librizzi F, Militello V, Gliozzi A, Leone M (2007) Amyloid fibrils formation and amorphous aggregation in concanavalin A. Biophys Chem 125:184-190 
28. Song X, Wei Z, Shaikh ZA (2015) Requirement of ER $\alpha$ and basal activities of EGFR and SrC kinase in Cd-induced activation of MAPK/ERK pathway in human breast cancer MCF-7 cells. Toxicol Appl Pharmacol 287:26-34

29. Dowling P, Clynes M (2011) Conditioned media from cell lines: a complementary model to clinical specimens for the discovery of disease-specific biomarkers. Proteomics 11:794-804

30. Wang S, Wang H, Guo H, Kang L, Gao X, Hu L (2011) Neuroprotection of Scutellarin is mediated by inhibition of microglial inflammatory activation. Neuroscience 185:150-160

31. Liu R-P, Zou M, Wang J-Y, Zhu J-J, Lai J-M, Zhou L-L, Chen S-F, Zhang X, Zhu J-H (2014) Paroxetine ameliorates lipopolysaccharide-induced microglia activation via differential regulation of MAPK signaling. J Neuroinflammation 11:47

32. Feng $Y$, Wang $X-P$, Yang $S-G$, Wang $Y-J$, Zhang $X$, Du X-T, Sun X-X, Zhao $M$, Huang L, Liu R$\mathrm{T}$ (2009) Resveratrol inhibits beta-amyloid oligomeric cytotoxicity but does not prevent oligomer formation. Neurotoxicology 30:986-995

33. Dai X, Hou W, Sun Y, Gao Z, Zhu S, Jiang Z (2015) Chitosan oligosaccharides inhibit/disaggregate fibrils and attenuate amyloid $\beta$-mediated neurotoxicity. Int J Mol Sci 16:10526-10536

34. Zhong L-M, Zong $Y$, Sun L, Guo J-Z, Zhang W, He Y, Song R, Wang W-M, Xiao C-J, Lu D (2012) Resveratrol inhibits inflammatory responses via the mammalian target of rapamycin signaling pathway in cultured LPS-stimulated microglial cells. PloS one 7:e32195

35. Shih Y-T, Chen I-J, Wu Y-C, Lo Y-C (2011) San-Huang-Xie-Xin-Tang protects against activated microglia- and 6-OHDA-induced toxicity in neuronal SH-SY5Y cells. Evid Based Complement Alternat Med 2011:429384

36. Cohen E, Dillin A (2008) The insulin paradox: aging, proteotoxicity and neurodegeneration. Nat Rev Neurosci 9:759-767

37. Radak Z, Zhao Z, Goto S, Koltai E (2011) Age-associated neurodegeneration and oxidative damage to lipids, proteins and DNA. Mol Aspects Med 32:305-315

38. Shukla V, Mishra SK, Pant HC (2011) Oxidative stress in neurodegeneration. Adv Pharmacol Sci 1043:545-552

39. de Fátima Bezerra M, Jamison BY, Gomada Y, Borges KC, Correia RTP, Vattem DA (2014) Eugenia jambolana Lam. Increases lifespan and ameliorates experimentally induced neurodegeneration in C. elegans. Int J Appl Res Nat Prod 7:39-48

40. Azevêdo JC, Borges KC, Genovese MI, Correia RT, Vattem DA (2015) Neuroprotective effects of dried camu-camu (Myrciaria dubia HBK McVaugh) residue in C. elegans. Food Res Int 73:135-141 
41. Wu Y, Wu Z, Butko P, Christen Y, Lambert MP, Klein WL, Link CD, Luo Y (2006) Amyloid$\beta$-induced pathological behaviors are suppressed by Ginkgo biloba extract EGb 761 and ginkgolides in transgenic Caenorhabditis elegans. J Neurosci 26:13102-13113

42. Dostal V, Roberts CM, Link CD (2010) Genetic mechanisms of coffee extract protection in a Caenorhabditis elegans model of $\beta$-amyloid peptide toxicity. Genetics 186:857-866 


\section{Figures and captions}

Fig.1 HPLC-DAD chromatogram of a phenolic-enriched maple syrup extract (MSX) showing 37 compounds identified as follows: (1) 4-hydroxy-2-(hydroxymethyl)-5-methyl-3(2H)furanone, (2) 3,4-dihydro-5-(hydroxymethyl)pyran-2-one, (3) 5-(hydroxymethyl)furfural, (4) 2-hydroxy-3,4-dihydroxyacetophenone, (5) 4-(hydroxymethyl)-1,2-benzenediol, (6) catechol, (7) C-veratroylglycol, (8) threo,threo-1-[4-(2-hydroxy-2-(4-hydroxy-3methoxyphenyl)-1-(hydroxymethyl)ethoxy)-3-methoxyphenyl]-1,2,3-propanetriol, (9) 2,3-dihydroxy-1-(4-hydroxy-3,5-dimethoxyphenyl)-1-propanone, (10) 4-acetylcatechol, (11) tyrosol, (12) catechaldehyde, (13) 1,2-diguaiacyl-1,3-propanediol, (14) 3',5'dimethoxy-4'-hydroxy-2-hydroxyacetophenone, (15) leptolepisol D, (16) 3,4-dihydroxy2-methylbenzadehyde, (17) vanillin, (18) fraxetin, (19) syringaldehyde, (20) syringenin, (21) scopoletin, (22) threo-guaiacylglycerol-6-O-4'-dihydroconiferyl alcohol, (23) erythroguaiacylglycerol-6-O-4'-dihydroconiferyl alcohol, (24) 5-(3",4"'-dimethoxyphenyl)-3hydroxy-3-(4'-hydroxy-3'-methoxybenzyl)-4-(hydroxymethyl) dihydrofuran-2-one, (25) 1-(2,3,4-trihydroxy-5-methylphenyl)ethanone, (26) erythro-1-(4-hydroxy-3methoxyphenyl)-2-[4-(3-hydroxypropyl)-2,6-dimethoxyphenoxy]-1,3-propanediol, (27) icariside E4, (28) 3', 4', 5'-trihydroxyacetophenone, (29) dehydroconiferyl alcohol, (30) sakuraresinol, (31) secoisolariciresinol, (32) acernikol, 
(33) $(1 S, 2 R)-2-[2,6-d i m e t h o x y-4-[(1 S, 3 a R, 4 S, 6 a R)$-tetrahydro-4-(4-hydroxy-3,5dimethoxyphenyl)-1H,3H-furo[3,4-c]furan-1-yl]phenoxy]-1-(4-hydroxy-3methoxyphenyl)-1,3-propanediol, (34) 2-[4-[2,3-dihydro-3-(hydroxymethyl)-5-(3hydroxypropyl)-7-methoxy-2-benzofuranyl]-2,6-dimethoxyphenoxy]-1-(4-hydroxy-3methoxyphenyl)-1,3-propanediol, (35) 4,4'-dihydroxy-3,3',5,5'-tetramethoxystilbene, (36) 4,4'-dihydroxy-3,3',5'-trimethoxystilbene, and (37) (E)-3,3'-dimethoxy-4,4'dihydroxystilbene.

Fig. 2 (A) ThT binding assay showing the levels of $A \beta_{1-42}$ aggregation treated with different concentrations of MSX $(50-500 \mu \mathrm{g} / \mathrm{mL})$ or positive control resveratrol (RESV; at 50 $\mu \mathrm{g} / \mathrm{mL}$ ); (B) TEM analyses of $A \beta_{1-42}$ solution freshly mixed with $500 \mu \mathrm{g} / \mathrm{mL}$ MSX without incubation (left), $A \beta_{1-42}$ solution incubated for $5 d$ (middle) and $A \beta_{1-42}$ solution co-treated with $500 \mu \mathrm{g} / \mathrm{mL}$ MSX then incubated for $5 \mathrm{~d}$ (right).

Fig. 3 Circular dichroism (CD) measurement of native $A \beta$, aggregated $A \beta$, and $A \beta$ co-treated with MSX (500 ug/mL).

Fig.4 MSX reduced $\mathrm{H}_{2} \mathrm{O}_{2}$-induced oxidative stress in $\mathrm{BV}-2$ murine microglial cells. (B) BV-2 cells were pretreated with MSX (50 or $100 \mu \mathrm{g} / \mathrm{mL}$ ) for $24 \mathrm{~h}$ followed by $1 \mathrm{~h}$ of $\mathrm{H}_{2} \mathrm{O}_{2}(3 \mu \mathrm{g} / \mathrm{mL}$ ) treatment. The $\mathrm{H}_{2} \mathrm{O}_{2}$-induced ROS production was measured by using the DCFDA reagent. 
Fig. 5 BV-2 cells pretreated with MSX (50 or $100 \mu \mathrm{g} / \mathrm{mL}$ ) for $1 \mathrm{~h}$ followed by exposure to LPS (1 $\mu \mathrm{g} / \mathrm{mL}$ ) for $24 \mathrm{~h}$. The treated cell culture media were used to assay the amount of NOS (A), IL-6 (B), PGE 2 (C), and TNF $\alpha$ (D) production. Resveratrol (RESV, at $5 \mu \mathrm{g} / \mathrm{mL}$ ) served as the positive control for all the assays. Data are presented as means \pm SDs of three independent experiments.

Fig. 6 MSX reduced murine BV-2 microglia-mediated neurotoxicity of human SH-SY5Y neuron cells. BV-2 cells were treated with MSX (50 and $100 \mu \mathrm{g} / \mathrm{mL}$ ) or resveratrol (RESV, 5 $\mu \mathrm{g} / \mathrm{mL}$; positive control) for $1 \mathrm{~h}$ prior to exposure to LPS $(1 \mu \mathrm{g} / \mathrm{mL})$ for $24 \mathrm{~h}$. The conditioned media from the BV-2 were collected and added to SH-SY5Y cells. After incubation for $24 \mathrm{~h}$, cell viability of SH-SY5Y cells were measured by the CTG 2.0 assay. All data points were compared to the LPS column.

Fig. 7 Mobility curves of transgenic (CL4176) C. elegans $20 \mathrm{~h}$ post $A \beta_{1-42}$ induction of muscular paralysis at $25^{\circ} \mathrm{C}$. Kaplan-Meier mobility plots of C. elegans worms fed on (A) Control (NGM); (B) MSX: (NGM + $10 \mu \mathrm{g} / \mathrm{mL} M S X)$. 
Fig. 1

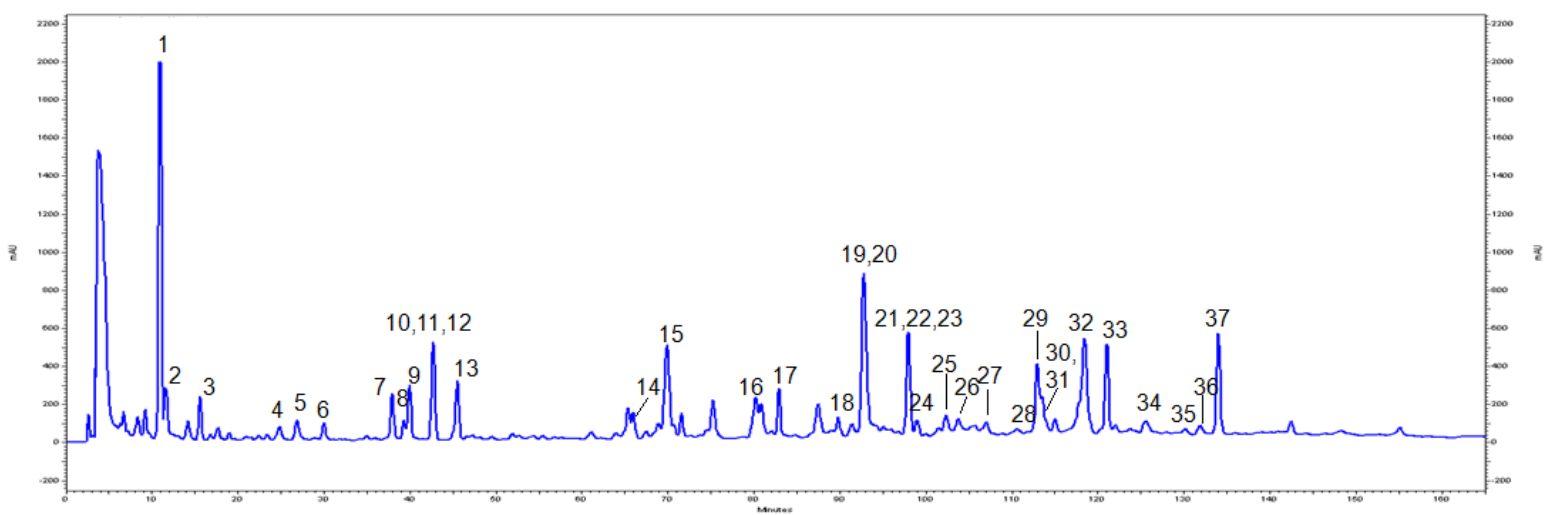


Fig. 2

(A)

(B)
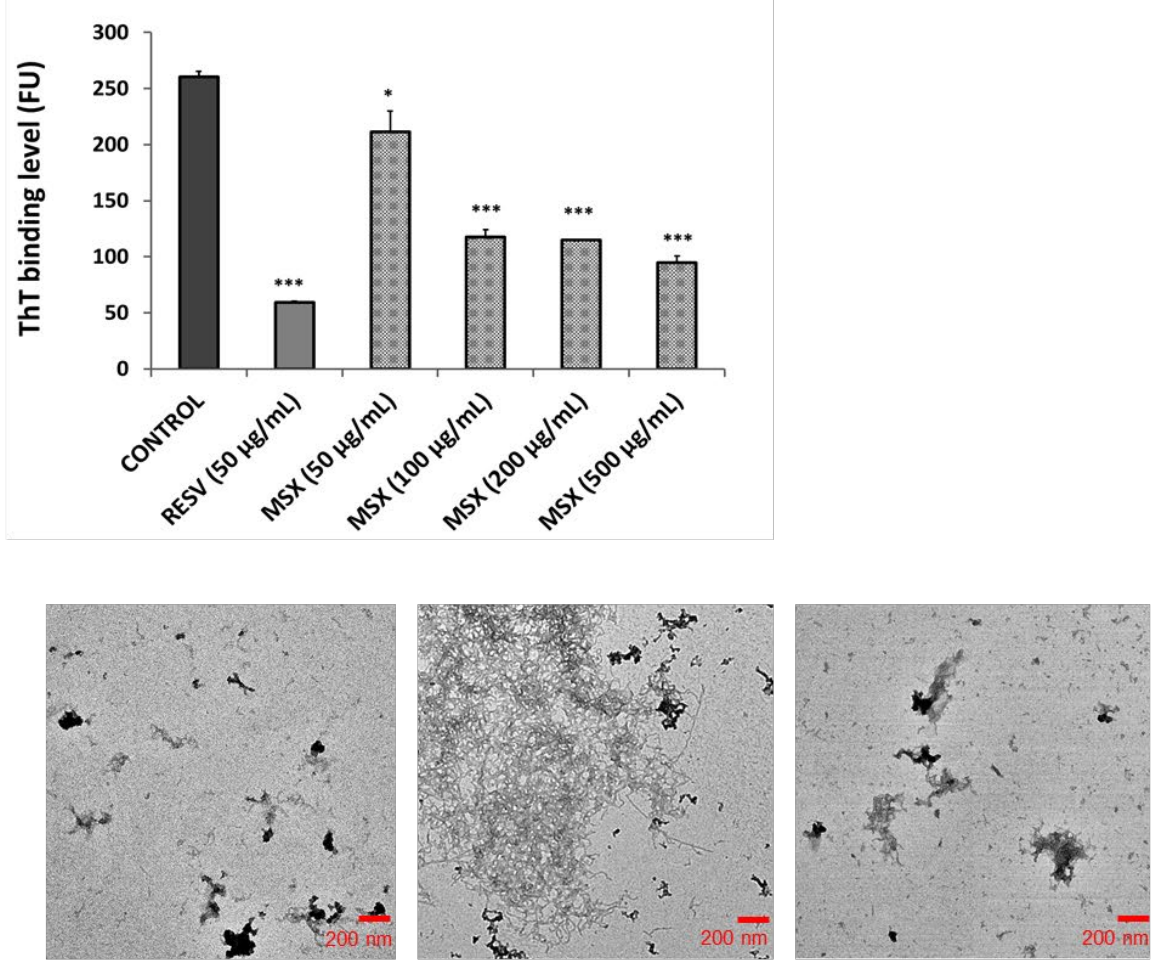

$A \beta_{1-42}$

$\operatorname{MSX}(500 \mu \mathrm{g} / \mathrm{mL})$

$+$

Incubation (5 d)

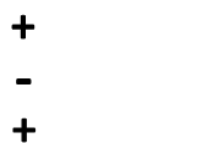

$+$

$+$

$+$ 
Fig. 3

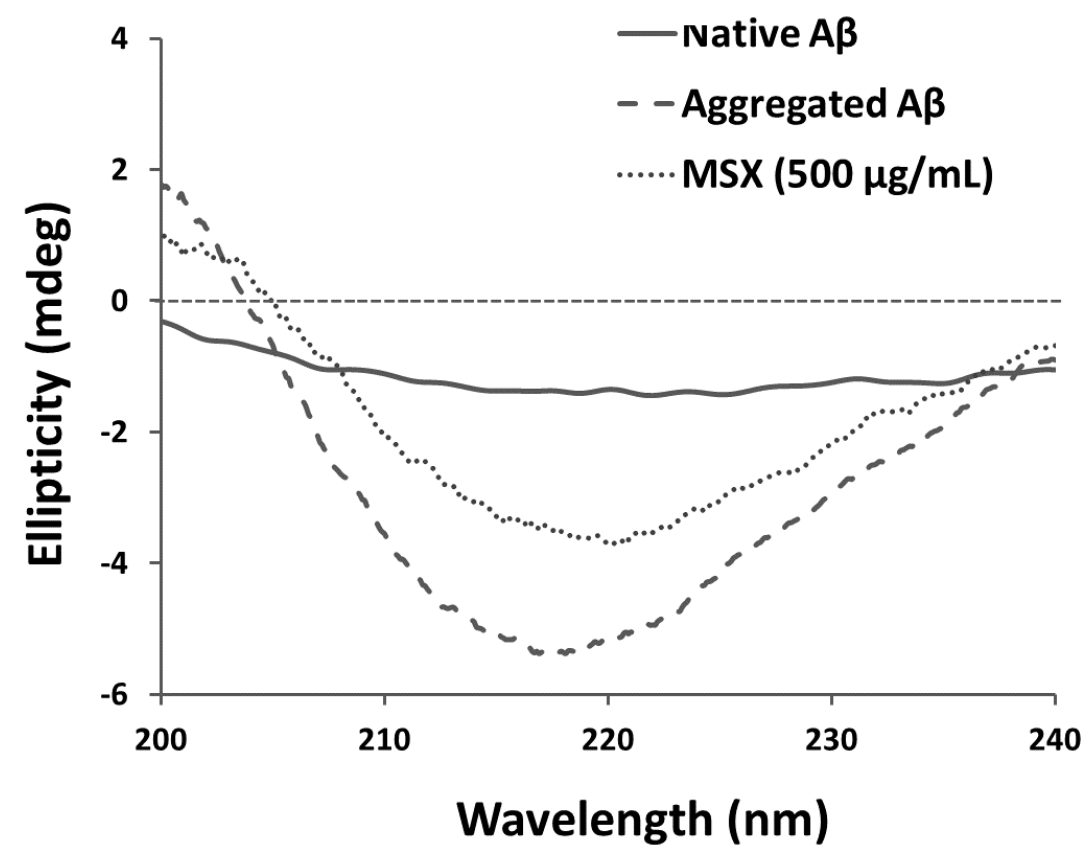


Fig. 4

(A)

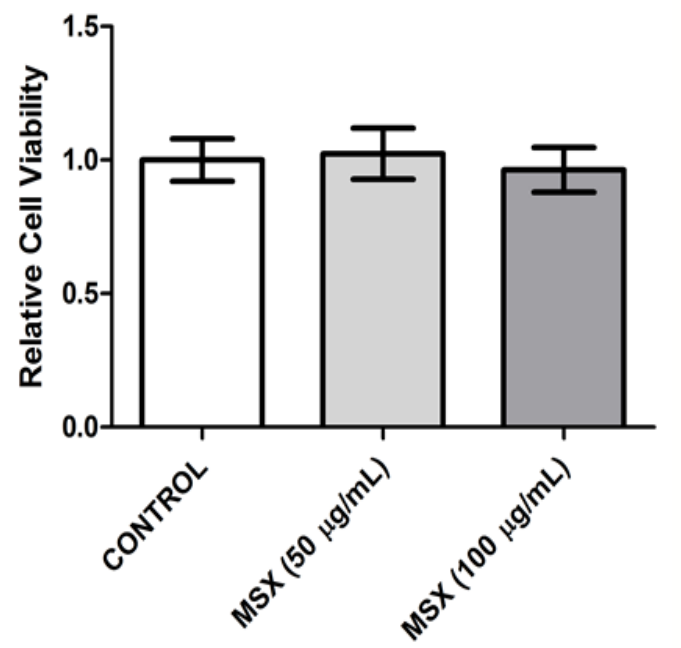

(B)

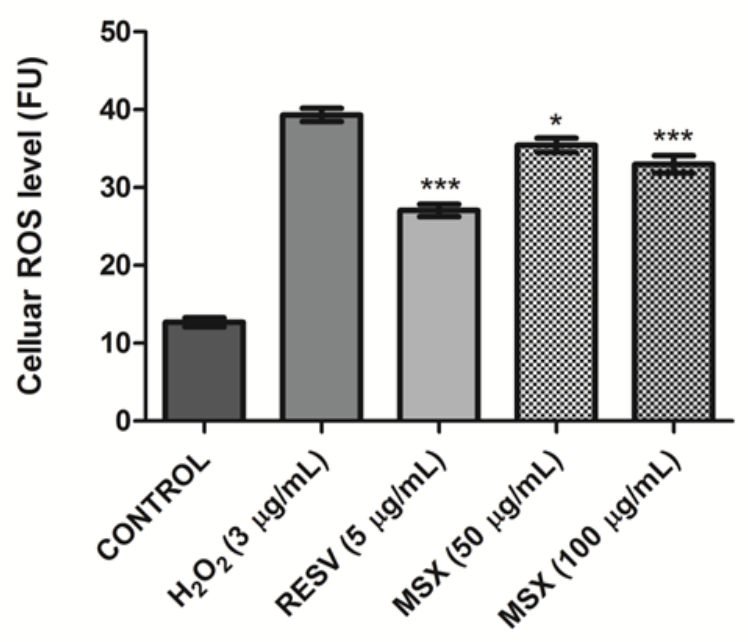


Fig. 5

(A)

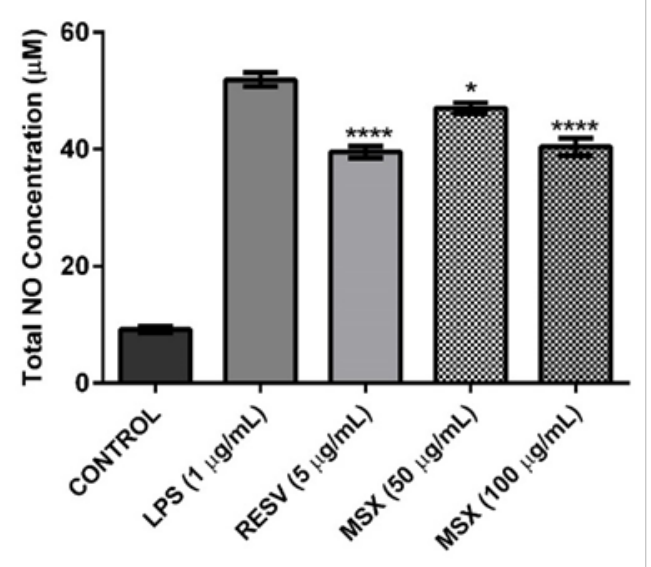

(C)

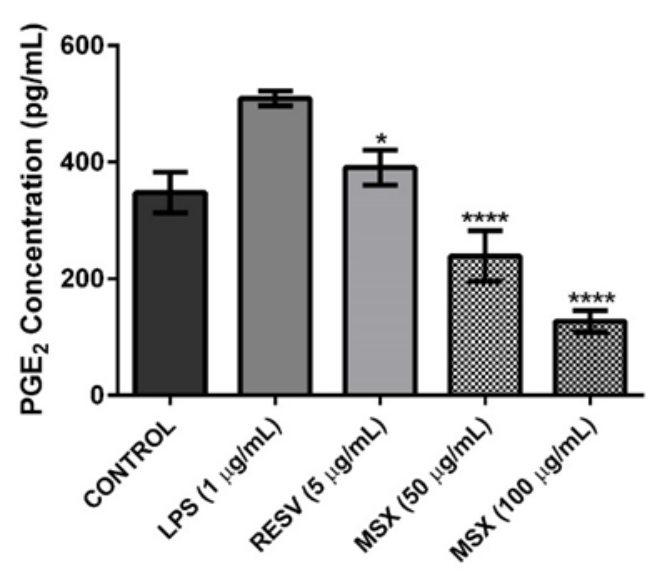

(B)

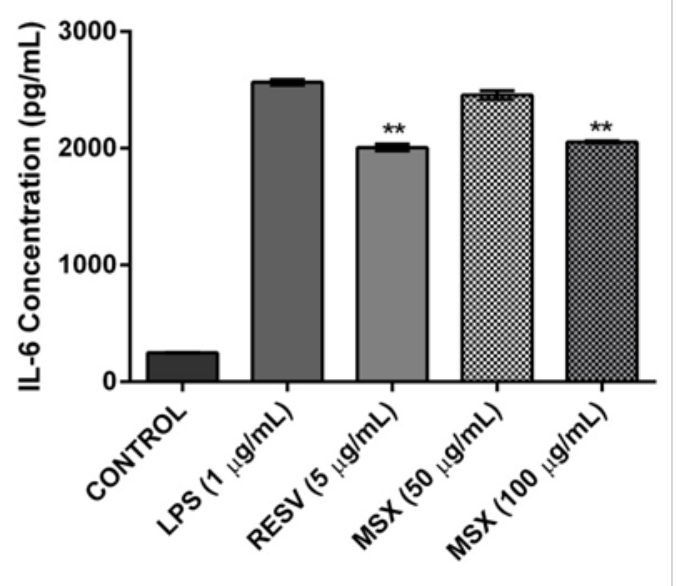

(D)

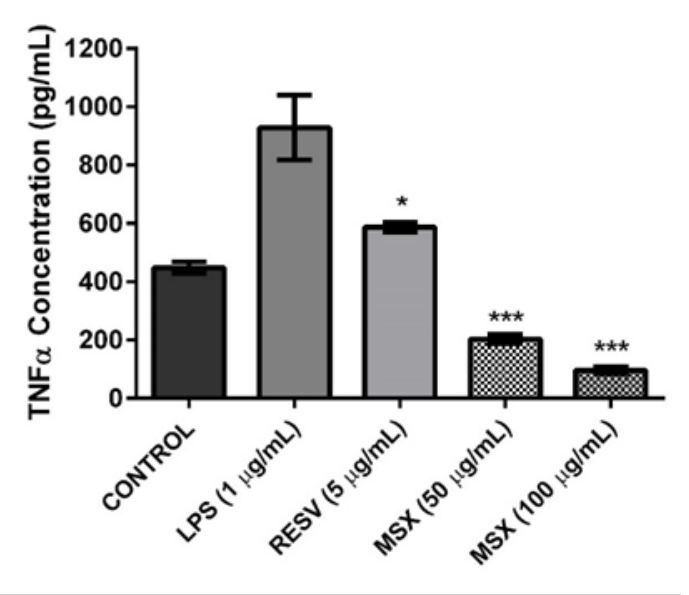


Fig. 6

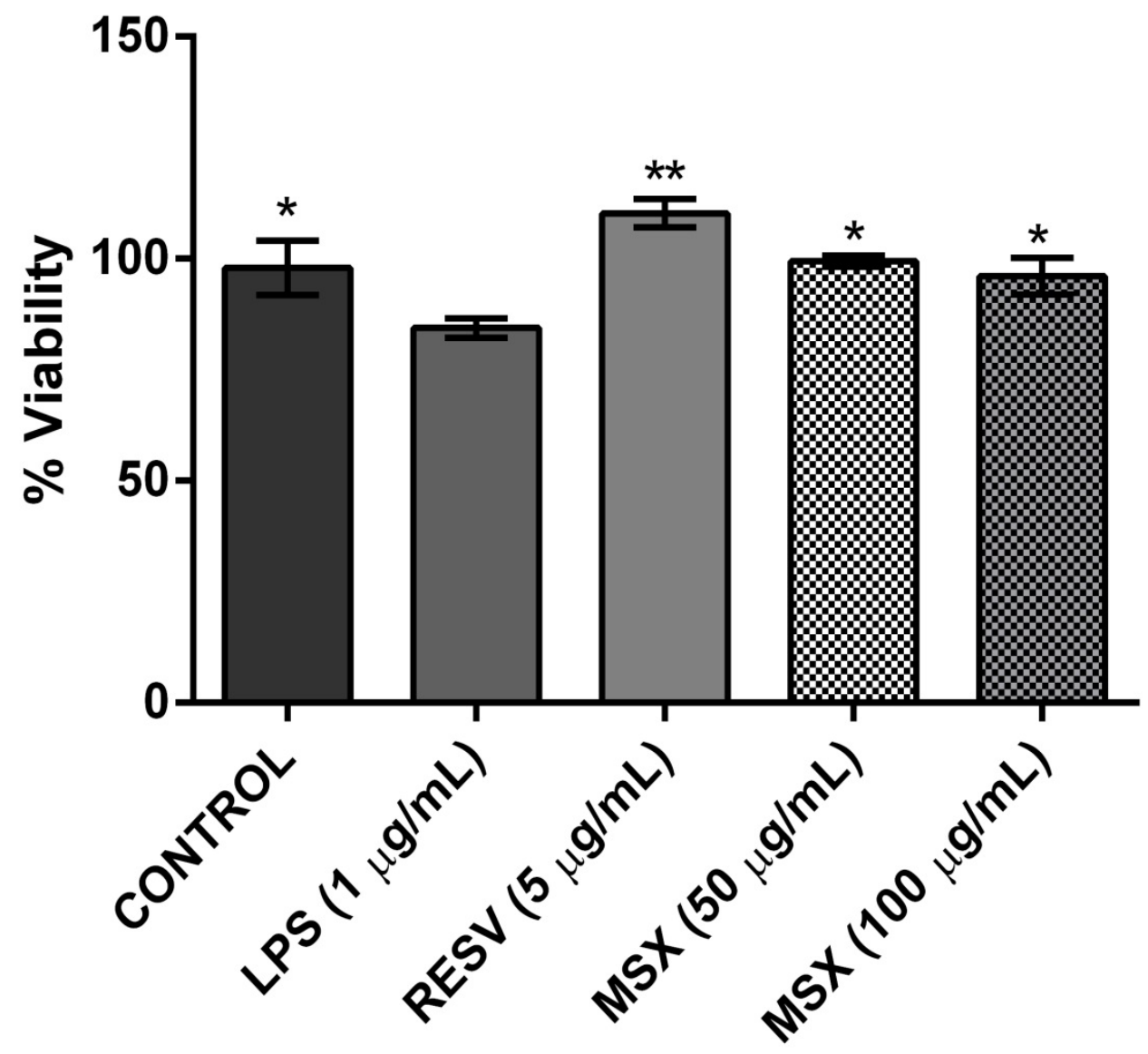


Fig. 7
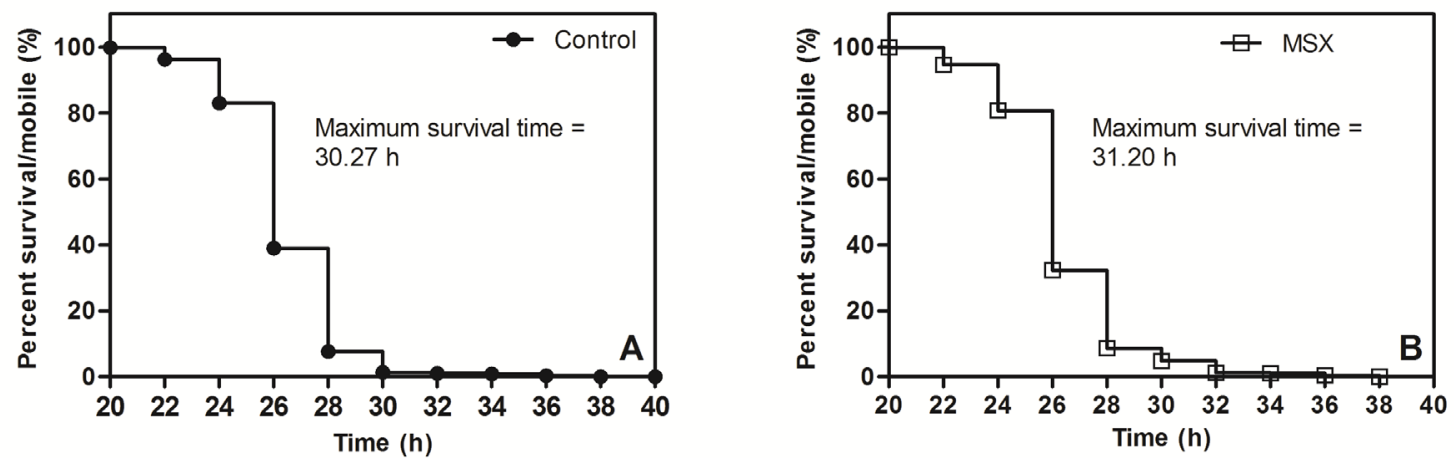
Table 1. Measurement of zeta potential, particle size distribution, and $\beta$-sheet content of native $A \beta_{1-42}$, aggregated $A \beta_{1-42}$ and $M S X(500 \mu \mathrm{g} / \mathrm{mL})$ treated $A \beta_{1-42}$. (n.d. = not detected) .

\begin{tabular}{lccc}
\hline & Native $A \beta_{1-42}$ & Aggregated A $\beta_{1-42}$ & MSX (500 $\mathrm{\mu g} / \mathrm{mL})$ \\
\hline Zeta Potential (mV) & -4.58 & -22.4 & -17.6 \\
Particle Size (d.nm) & n.d. & $317.9(86.4 \%)$ & $39.84(77.0 \%)$ \\
& & $3.088(13.6 \%)$ & $4.838(23.0 \%)$ \\
\hline -sheet content (\%) & 18.8 & 59.6 & 49.6 \\
\hline
\end{tabular}


Table 2. Survival (mean, median, and maximum) of (CL4176) C. elegans worms treated with MSX $(10 \mu \mathrm{g} / \mathrm{mL})$ post $A \beta_{1-42}$ induction of muscular paralysis at $25^{\circ} \mathrm{C}$. [* $\mathrm{p}<0.05$; Log rank test (Mantel (ox)].

\begin{tabular}{lccc}
\hline $\begin{array}{l}\text { Treatment } \\
(10 \mu \mathrm{g} / \mathrm{mL})\end{array}$ & Mean & Median & Maximum \\
\hline Control $(\mathrm{n}>1000)$ & 29.60 & 26.00 & 30.27 \\
MSX $(n>1000)$ & $26.50^{*}$ & 26.00 & $31.20^{*}$ \\
\hline
\end{tabular}




\section{CONCLUSIONARY REMARKS}

The modulation of inflammation and the targets critical to the pathogenesis of neurodegenerative and metabolic diseases through the use of functional foods appears to be a somewhat distant reality. Manuscript 1 demonstrated that PE modulated hippocampal inflammation, reduced AD-associated genes (APP, GSK3 3 , and MAPT), reduced body, liver, and white adipose tissue weight while also improving hepatic metabolic function in diet-induced obese mice. Moreover, in manuscript 4 the BBB-permeable metabolites, urolithins, prevented reactive microgliosis and microglia mediated neuron death.

MSX was thoroughly evaluated for similar immunomodulatory effects in vivo and in vitro. Manuscript 2 evaluated the anti-neuroinflammatory effects of MSX in a diet-induced obesity rodent model and more importantly, showed a potential for modulating leptin receptor signaling within the hippocampus. MSX also showed beneficial effects in preventing liver lipid accumulation and promoted lipid metabolism which prompted further investigation through a more robust study in manuscript 3.

This larger study used a more compositionally realistic western-style diet which included the addition of fructose and glucose in drinking water. MSX surprisingly had the opposite effect than we hypothesized and exacerbated hepatic lipid metabolism and transport. One possible explanation aside from the detrimental effects of fructose consumption on liver lipid accumulation and function would be the stereospecific production of (+) EL over (-) EL. The current body of research suggests that the $(+)$ and even racemic mixture may augment hepatic de novo lipogenesis. (-) SECO, (-) ED, and (-) EL potentiated the effects of a similar diet-induced obesity model. 
Given that microbial transformation of ED to EL is stereospecific (i.e. (+) ED can only be converted to (+) EL), microbial dysbiosis due to HFD consumption with fructose, may promote the growth of unique bacteria capable of this ED to EL stereospecific transformation. Confirmation of which form of EL exists should be determined by LC-MS analysis of week 18 sera. Additional studies involving only the biologically relevant entities are still needed to explore the toxicological effects of simultaneous high fat-high fructose consumption with the microbial metabolites of MSX.

As with PE and the discovery of urolithins, the methodologies used to determine the biologically present microbial metabolites are needed to identify the active constituents of MSX. Fermentation studies using an artificial gut system with MSX incubated in human fecal innocula at varying $\mathrm{pH}$ conditions should be used to model the environment of the gut. The end result should produce microbial metabolites such as Enterolignans (ED and EL) and potentially new molecular entities. With these metabolites identified, passive and active permeability of these metabolites through the intestinal epithelial barrier and the blood brain barrier should be quantified to ascertain which compounds may enter hepatic circulation and the brain. With a more defined and biologically relevant pool of metabolites, mechanism of action studies involving the toll-like receptor 4 - NfKB pathway as well as the possible effects of astrocyte mediated leptin receptor signaling should be explored.

Manuscript 5 demonstrated MSX significantly attenuated pro-inflammatory cytokines and pre-mature cell death in human monocytes, immortalized macrophages, immortalized neurons and even murine microglia. Future studies once more should screen for only the BBB permeable constituents of MSX to determine a mechanism of action. 
While PE continues to be heavily researched for a number of disease states, MSX is still an interesting source of beneficial compounds and metabolites. Future studies involving MSX should focus on determining the bioactive and bioavailable metabolites present in mammals. Given the complexity of MSX, the stereospecific microbial conversion of EL represents just one possibility apart from the simple addition of fructose that could support our findings from the initial pilot study in manuscript 2 and the larger multi-dose study of manuscript 3. In closing, this research will contribute to the ever growing body of research aimed at identifying and characterizing natural products with immunomodulatory activity. More importantly, this work underscores the importance of gut microbial metabolism as a significant source of beneficial and harmful metabolites capable of promoting or worsening health. 